\title{
FEASIBILITY OF MANIPULATING CORRELATED COLOR TEMPERATURES WITH A PHOSPHOR CONVERTED HIGH-POWERED LIGHT EMITTING DIODE WHITE LIGHT SOURCE
}

\author{
A Thesis \\ Presented to \\ the Faculty of California Polytechnic State University \\ San Luis Obispo
}

\begin{abstract}
In Partial Fulfillment
of the Requirements for the Degree

Master of Science in General Engineering with Specialization in Materials Engineering

by

Matthew Michael Little

June, 2010
\end{abstract}


(C) 2010

Matthew Michael Little

ALL RIGHTS RESERVED

ii |P a g e 


\section{COMMITTEE MEMBERSHIP}

TITLE:

AUTHOR:

DATE SUBMITTED:

COMMITTEE CHAIR:

COMMITTEE MEMBER:

COMMITTEE MEMBER:
Feasibility of manipulating correlated color temperatures with a phosphor converted high-powered light emitting diode white light source

Matthew Michael Little

June, 2010

Dr. Richard Savage, Materials Engineering Professor

Dr. Kathy Chen, Materials Engineering Professor

Dr. Linda Vanasupa, Materials Engineering Professor 


\begin{abstract}
Feasibility of manipulating correlated color temperatures with a phosphor converted high-powered light emitting diode white light source

Matthew Michael Little
\end{abstract}

In this thesis we examine the feasibility of developing a white light source capable of producing colors between 2500 and 7500 Kelvin on the black-body radiator spectrum by simply adjusting amperage to a blue and ultraviolet (UV) light emitting diode (LED). The purpose of a lighting source of this nature is to better replicate daylight inside a building at a given time of day. This study analyzes the proposed light source using a $385 \mathrm{~nm}$ UV LED, a $457 \mathrm{~nm}$ blue LED, a $479 \mathrm{~nm}$ blue LED, a $562 \mathrm{~nm}$ peak cerium doped yttrium aluminum garnet (YAG:Ce) phosphor, and a $647 \mathrm{~nm}$ peak selenium doped zinc sulfide (ZnS:Se) phosphor.

Our approach to this study initially examined optical performance of yellow-emitting phosphor (YAG:Ce) positioned at specific distances above a blue LED using polydimethylsiloxane (PDMS) as a substrate. An understanding of how phosphor concentration within the PDMS, the thickness of the PDMS, and how substrate distance from the LED die affected light intensity and color values (determined quantitatively by utilizing the $1931 \mathrm{CIE} 2^{\circ}$ Standard Observer) enabled equations to be developed for various lens designs to efficiently produce white light using a $457 \mathrm{~nm}$ peak wavelength LED. The combination of two luminescent sources (457 nm LED and YAG:Ce) 
provided a linear trend on the 1931 CIE diagram which required a red illumination source to obtain Kelvin values from 2500 to 7500 . Red-emitting phosphor ( $\mathrm{ZnS}: \mathrm{Se}$ ), selected to compliment the system, was dispersed with YAG:Ce throughout PDMS where they were stimulated with a blue LED thereby enabling all desired Kelvin values with differing concentration lenses. Stimulating ZnS:Se with the addition of a UV LED did not provide the ability to change the color value of the set up to the degree required. Many other factors resulted in the decision to remove the UV LED contribution from the multi-Kelvin light source design. The final design incorporated a combination of ZnS:Se and YAG:Ce stimulated with a blue LED to obtain a 2500 Kelvin value. A separate blue LED provides the means to obtain 7500 Kelvin light and the other color values in between, with a linear approximation, by adjusting the amperages of both LEDs.

In addition to investigating the feasibility of obtaining the Kelvin values from 2500 to 7500 , this thesis also examined the problem of $\mathrm{ZnS}$ :Se's inability to cure in PDMS and a method to create a lens shape to provide equal color values at all points above a phosphor converted LED source. ZnS:Se was found to be curable in PDMS if first coated with a low viscosity silicon oil prior to dispersion within PDMS. The lens configuration consists of phosphors equally distributed in PDMS and cured in the shape of a Gaussian distribution unique to multiple factors in LED-based white light design.

Keywords: White High-Powered Light Emitting Diode (LED), Correlated Color Temperature (CCT), Kelvin, Phosphor, Photoluminescence, YAG:Ce, ZnS:Se, BAM:Eu, Polydimethylsiloxane (PDMS) 


\section{ACKNOWLEDGEMENTS}

I would like to acknowledge the advice, guidance, and support of Dr. Richard Savage throughout the entirety of this thesis which enabled me to develop an understanding of the subject. I am forever grateful for all I have gained by working with him. I also thank the members of my committee: Dr. Kathy Chen for her suggestions and concerns regarding various works and Dr. Linda Vanasupa for her valuable instructions on how to approach and design experiments.

I appreciate support provided by Steve Meredith through multiple discussions, which better defined my Thesis direction, and his contributions to the initial apparatus design and programming. I am thankful to have had the many conversations with Nic Vickers which helped to tackle important obstacles along the way.

I thank Dr. Jianbiao Pan and his students, notably Jason Maynard, who dedicated time to the mounting and wire bonding of LEDs on a unique structure.

Special thanks to General LED for financial support and project definition. In particular I would like to thank Bill Ratcliffe for opening my eyes to the LED industry and putting experiments into perspective with actual products.

Lastly, I offer my sincerest gratitude and blessings to family, friends, and all of those who supported me in any respect during the completion of this project. 


\section{TABLE OF CONTENTS}

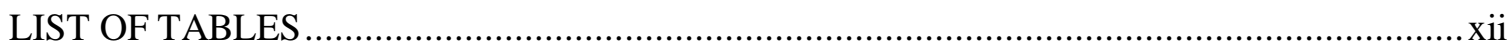

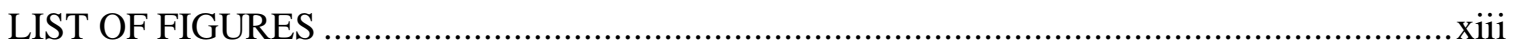

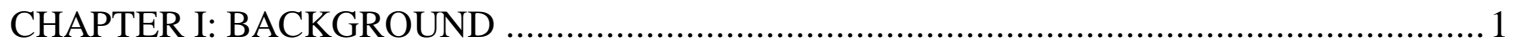

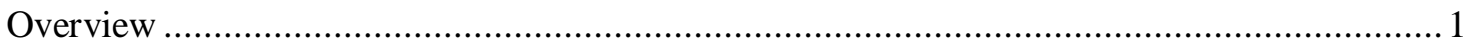

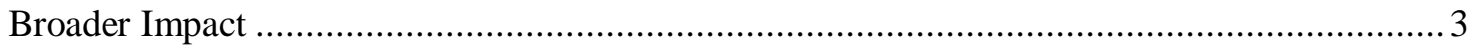

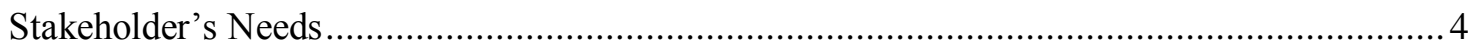

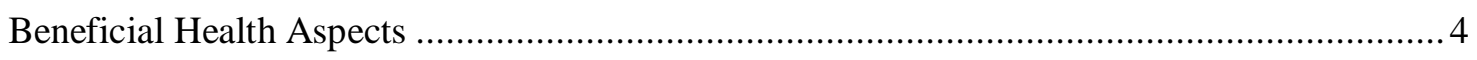

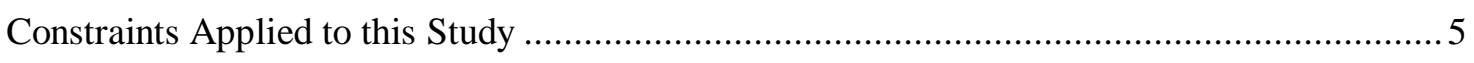

CHAPTER II: INTRODUCTION TO PHOSPHORS ......................................................... 8

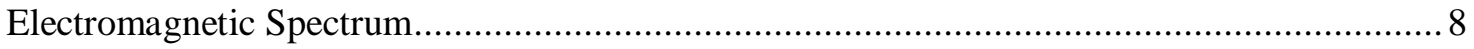

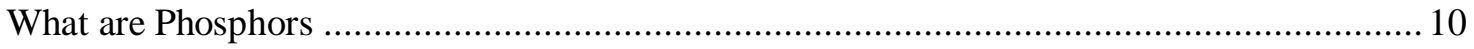

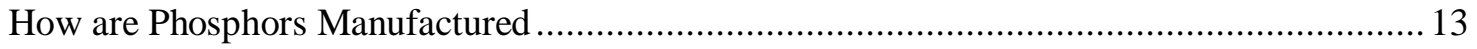

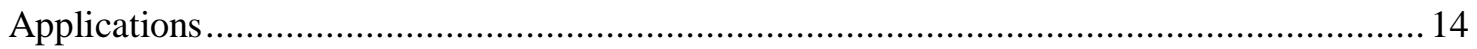

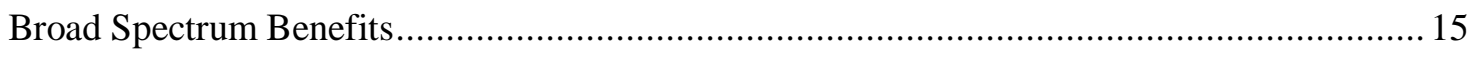

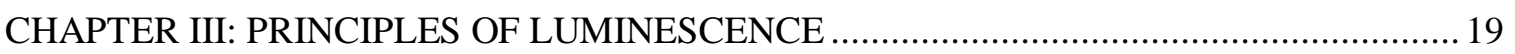

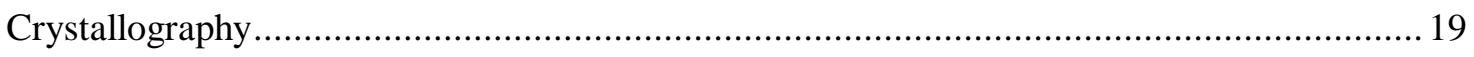

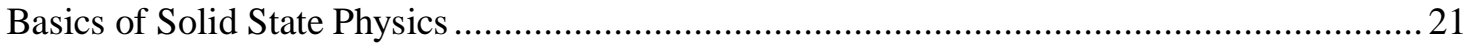

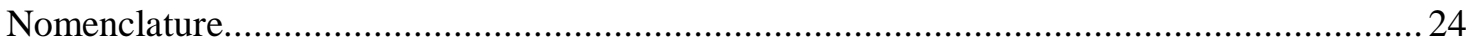

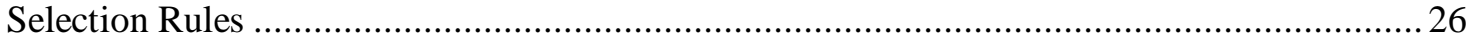

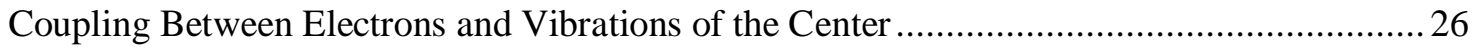

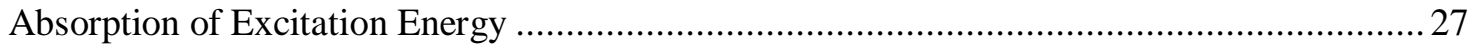

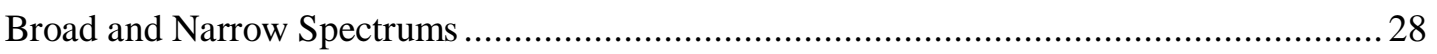

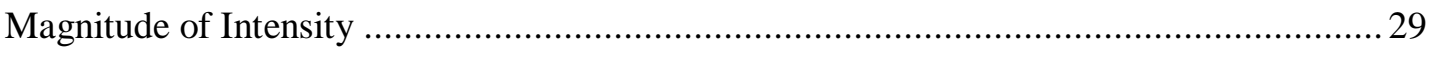

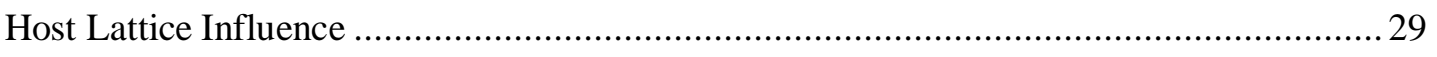

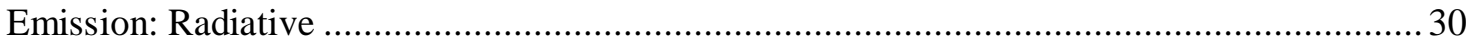

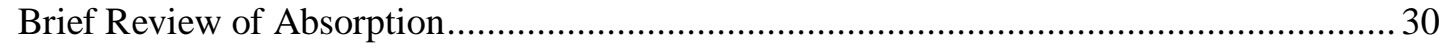

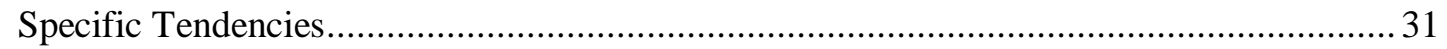

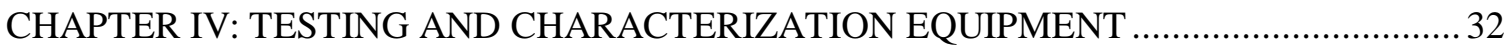

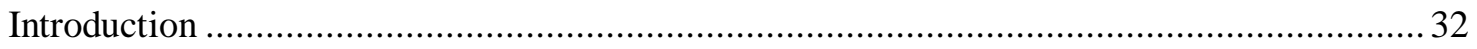

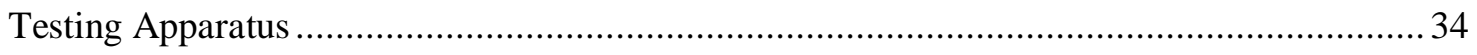




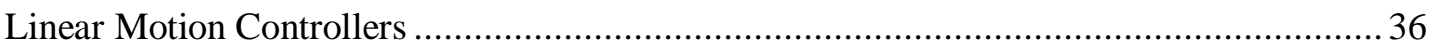

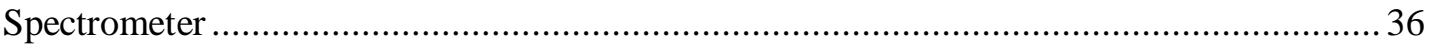

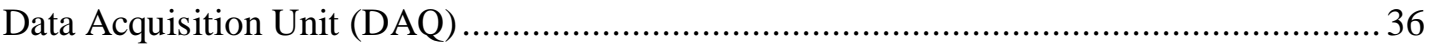

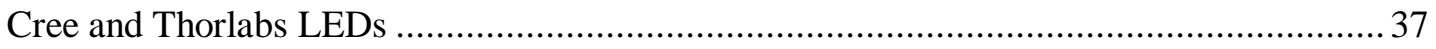

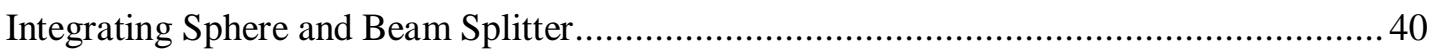

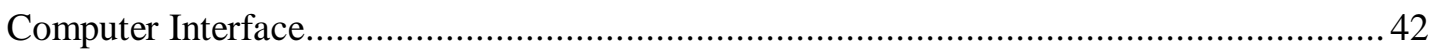

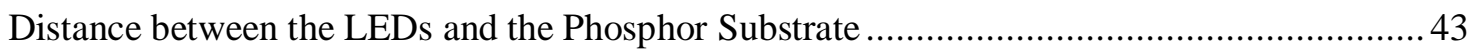

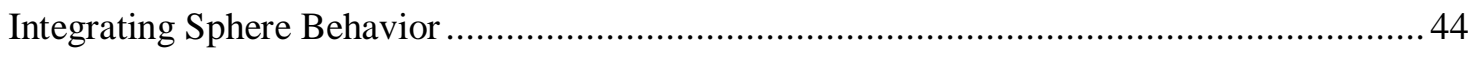

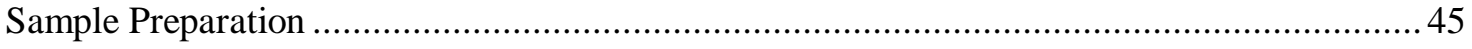

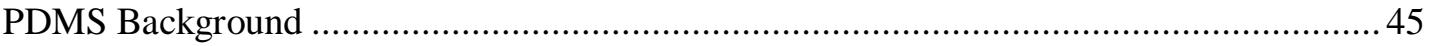

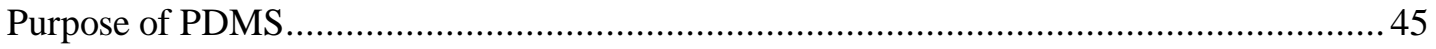

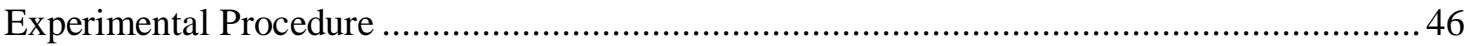

CHAPTER V: OBSERVED BEHAVIORS USING A 457 nm THORLABS BLUE LED

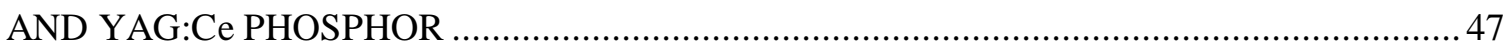

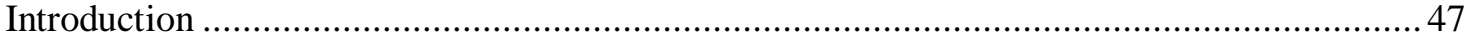

Crystallography and Photoluminescence of YAG:Ce ....................................................... 47

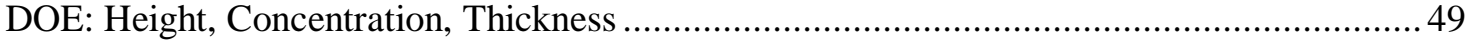

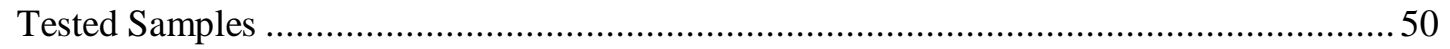

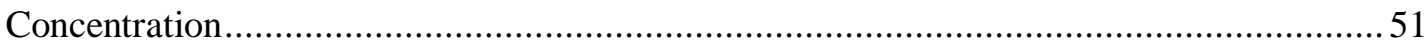

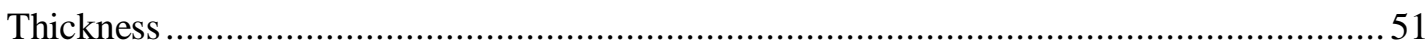

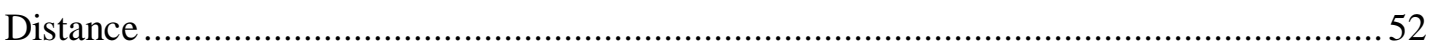

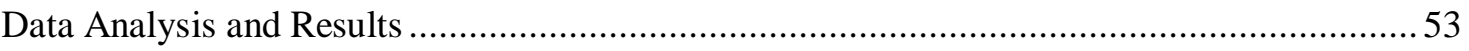

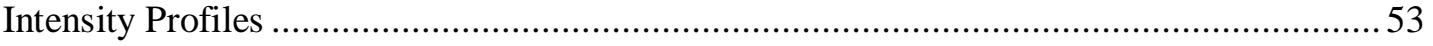

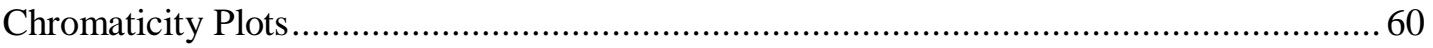

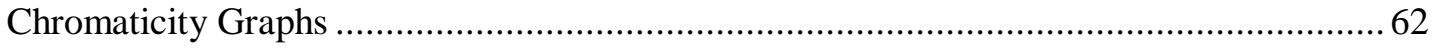

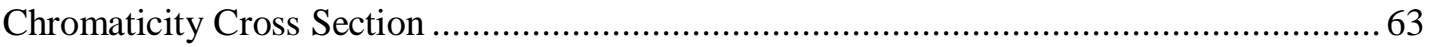

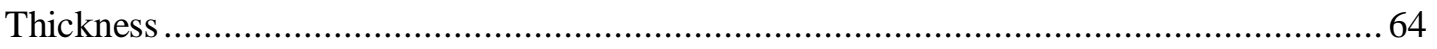

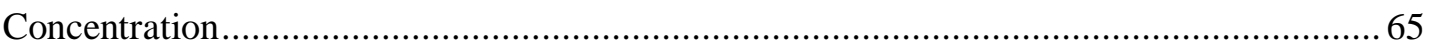

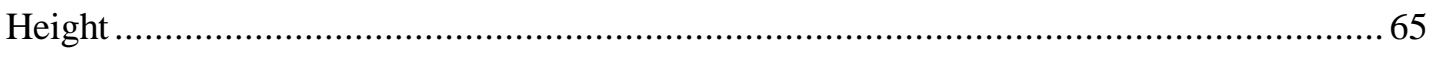

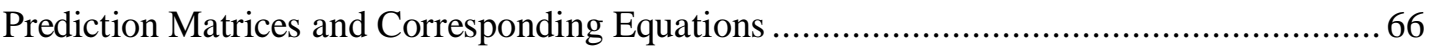

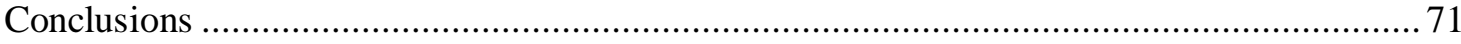




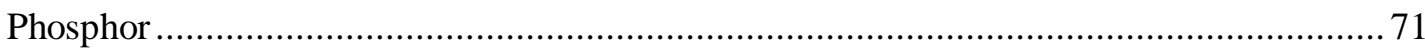

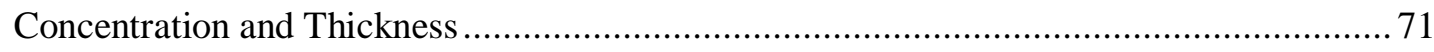

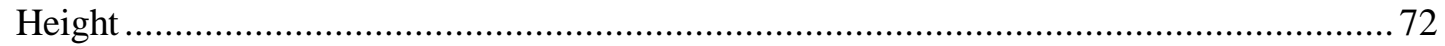

CHAPTER VI: CREATING A MULTI-KELVIN WHITE SOURCE USING A BLUE AND UV LED IN CONJUNCTION WITH YAG:Ce AND ZnS:Se PHOSPHORS ............................ 73

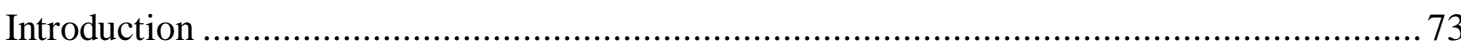

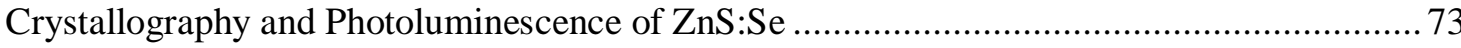

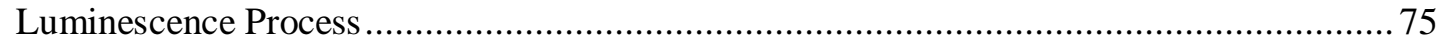

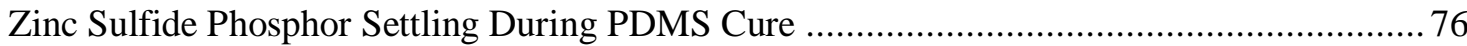

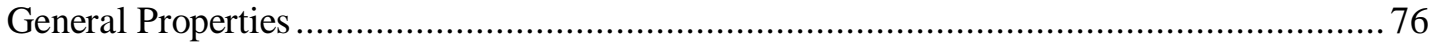

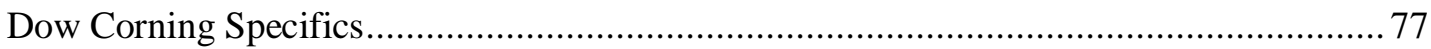

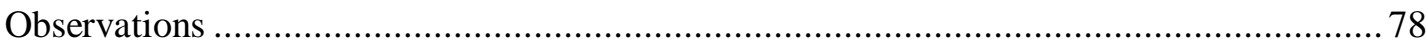

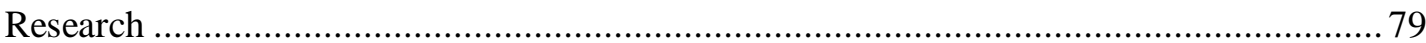

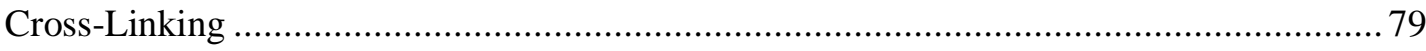

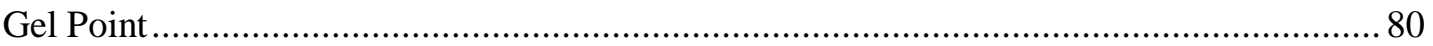

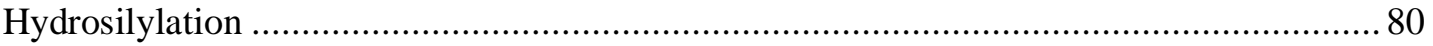

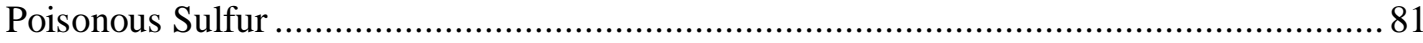

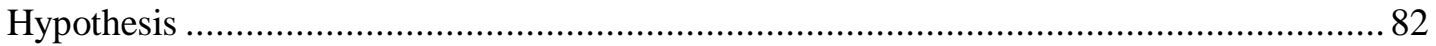

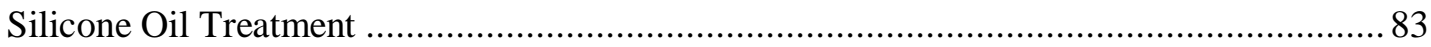

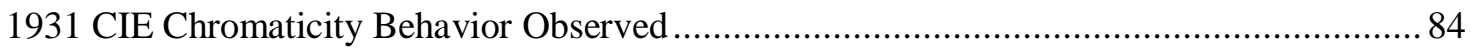

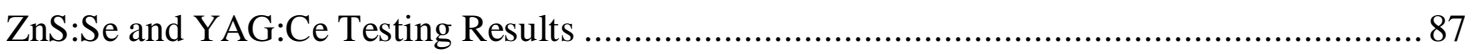

CHAPTER VII: METHOD TO APPROXMIATE KELVIN VALUES BETWEEN 2500

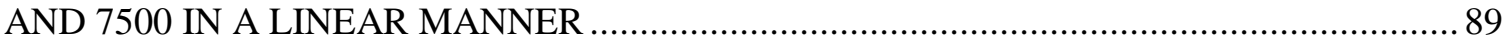

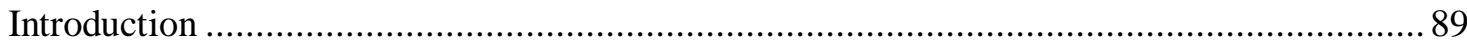

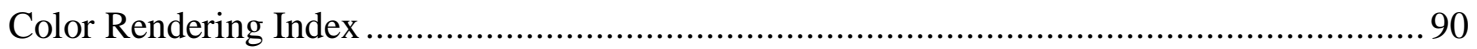

Variable White Light with Two LEDs (Blue and UV) and a Phosphor Substrate................... 90

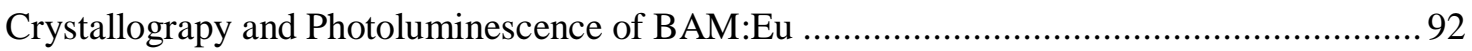

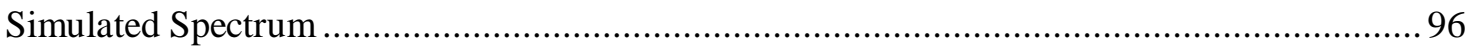

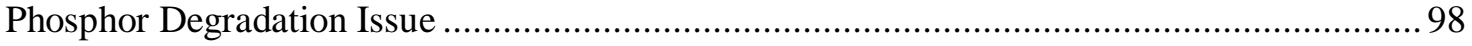

CHAPTER VIII: CONSIDERATION OF CHROMATICITY GRADIENTS AND THE INTENSITY RELATIONSHIP WITH KELVIN VALUES OF THE FINAL DESIGN ........... 104

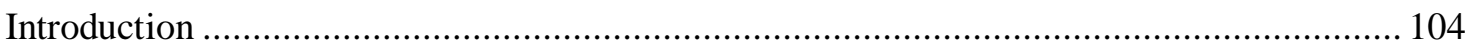




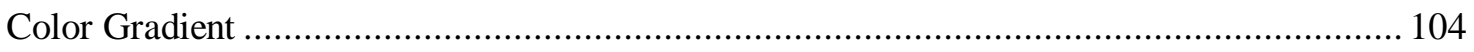

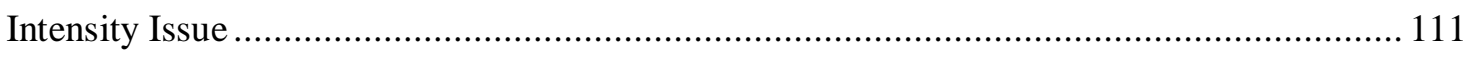

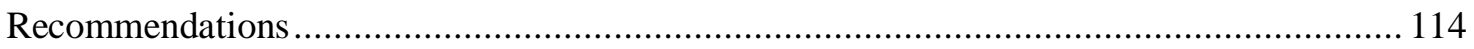

CHAPTER IX: RECOMMENDATIONS FOR FUTURE WORK ........................................... 117

Addressing Efficiency Issues with Phosphors Separate from the LED Die .......................... 117

Thin Layer Gaussian Profile ...................................................................................... 118

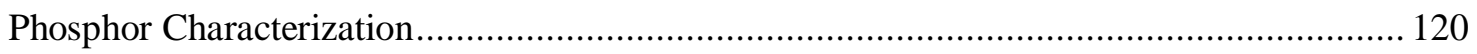

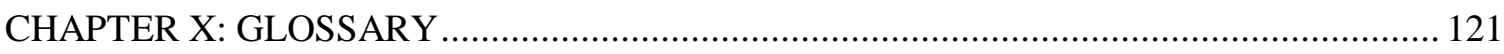

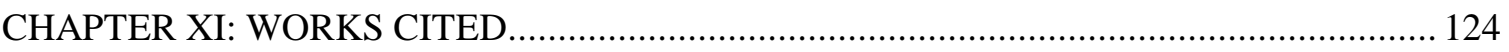

Appendix A: Method of Production (Pouring, Spin Coating, and Plasma Bonding) ................. 128

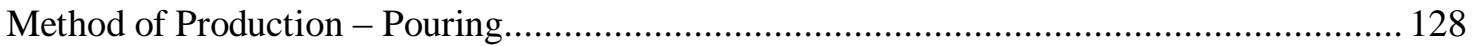

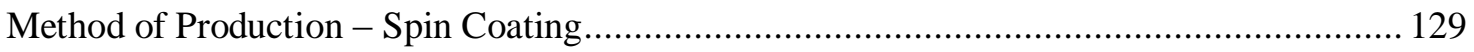

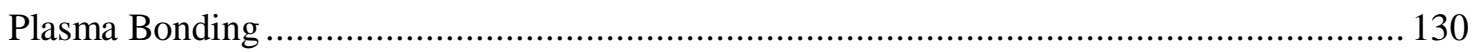

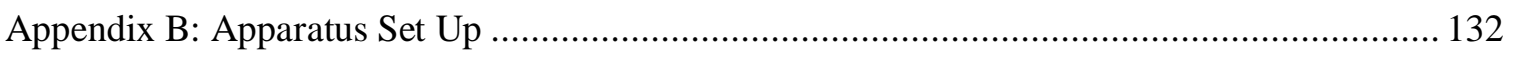

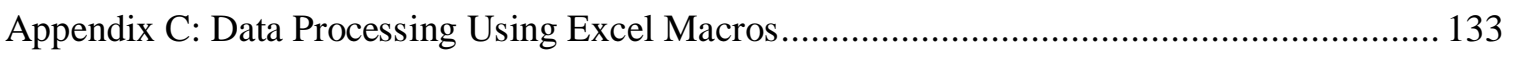

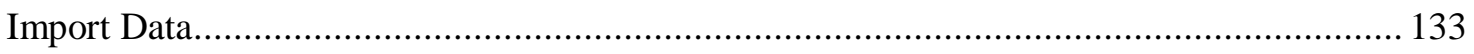

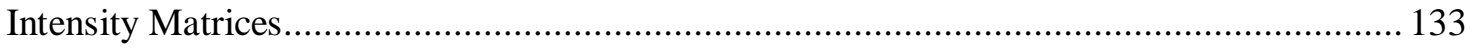

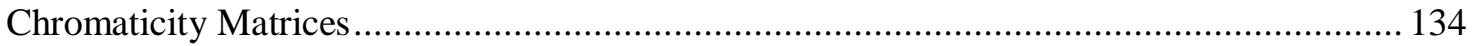

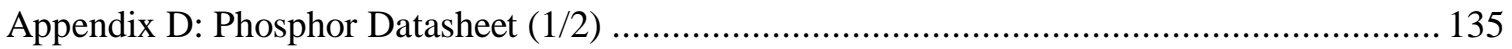

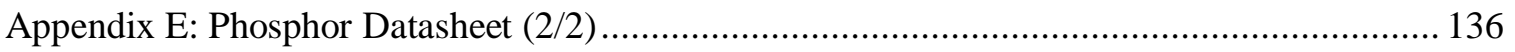

Appendix F: Predominantly Blue Intensity Profile of Various Concentration Substrates .......... 137

Appendix G: Predominantly Yellow Intensity Profile of Various Concentration Substrates...... 138

Appendix H: Entire Visible Spectrum Intensity Profile of Various Concentration Substrates ... 139

Appendix I: X Chromaticity Comparison of Various Substrates Thicknesses .......................... 140

Appendix J: Y Chromaticity Comparison of Various Substrates Thicknesses ........................... 141

Appendix K: X Chromaticity Comparison of Various Height Experiments .............................. 142

Appendix L: Y Chromaticity Comparison of Various Height Experiments .............................. 143

Appendix M: X Chromaticity Plot of Thickness versus Concentration Directly Above LED .... 144

Appendix N: Y Chromaticity Plot of Thickness versus Concentration Directly Above LED .... 145

Appendix O: Y Chromaticity Plot of Thickness versus Concentration Offset Above LED ....... 146

Appendix P: X Chromaticity Plot of Height versus Thickness Directly Above LED ................ 147 
Appendix Q: Y Chromaticity Plot of Height versus Thickness Directly Above LED ................ 148

Appendix R: X Chromaticity Plot of Height versus Thickness Offset Above LED ................... 149

Appendix S: Y Chromaticity Plot of Height versus Thickness Offset Above LED ................... 150

Appendix T: X Chromaticity Plot of Height versus Concentration Directly Above LED .......... 151

Appendix U: Y Chromaticity Plot of Height versus Concentration Directly Above LED.......... 152

Appendix V: X Chromaticity Plot of Height versus Concentration Offset Above LED............. 153

Appendix W: Y Chromaticity Plot of Height versus Concentration Offset Above LED ............. 154

Appendix X: Chromaticity Values Measured over a Gaussian YAG:Ce/PDMS Lens with

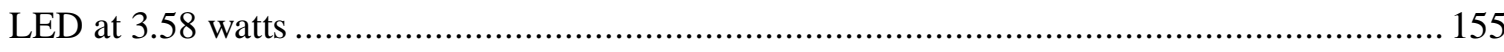

Appendix Y: Chromaticity Values Measured over a Gaussian YAG:Ce/PDMS Lens with

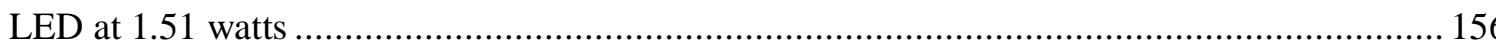




\section{LIST OF TABLES}

Table I - Important LEDs and Phosphors ........................................................................ 1

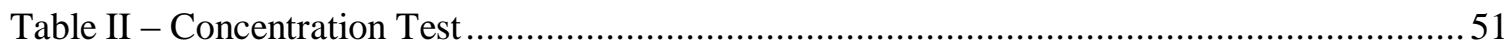

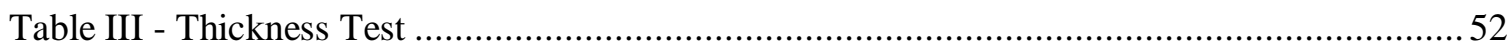

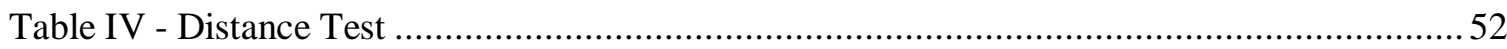

Table V - Thickness Comparison (Adjusted Total Intensity) .................................................5

Table VI - Concentration Comparison (Adjusted Total Intensity) ............................................59

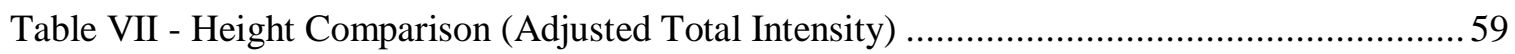

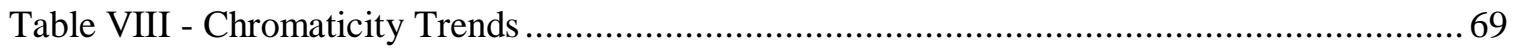




\section{LIST OF FIGURES}

Figure 1 - Depicts the color of light observed for a given Kelvin temperature displayed on the vertical axis $(5)$

Figure 2 - A representation of blue and UV LED sources both illuminating the same phosphor substrate to deliver the desired white light to an observer. The goal is to be able to adjust amperage to both LEDs in such a way which will result in a 5000 Kelvin change in color values to an observer of the phosphor substrate.

Figure 3 - A depiction of the electromagnetic spectrum from 0.1 angstroms to 1000 meters. A detailed view of the visible spectrum displays the colors observed at each wavelength from 380 to $770 \mathrm{~nm}_{(6)}$.

Figure 4 - The cross-sectional view of a low-power white LED. The LED is positioned at the bottom of the reflector cup and covered by yellow phosphor. Some of the blue light stimulates the phosphor to enable yellow photoluminescence. The combination of blue light, which did not interact with the phosphor, and yellow light emitted from that region appear white to the human eye ${ }_{(7)}$

Figure 5 - A zinc sulfide ( $\mathrm{ZnS}$ ) unit cell (represented by dashed lines) of zinc (small/green) and sulfur (large/red) atoms arranged in what is known as the wurtzite lattice. These cubic cells are repetitive throughout the entire substance $(10)$.

Figure 6 - In semiconductor materials point defects, such as vacancies, interstitial impurities, or antisite defects, provide the luminescent centers necessary for light emission. In other inorganic phosphors, such as YAG:Ce or BAM:Eu, substitution atoms become the luminescent center ${ }_{(10}$

Figure 7 - Three wavelengths of light (red, green, and blue) are incident upon a blue object. Red and green wavelengths are absorbed by the objects crystal lattice leaving blue light to reflect back toward an observer ${ }_{(14)}$.

Figure 8 - The left-hand side shows a crystalline region of a material (short range order) whereas the right-hand side represents an amorphous region. If much of the remainder of the material maintains the crystalline structure then long range order is observed ${ }_{(18)}$.

Figure 9 - Sketch of the potential energy per solid pair of $\mathrm{NaCl}$ (salt). Equilibrium exists at the lowest potential energy level $\left(r_{0}=0.28 \mathrm{~nm}\right)$, two ions infinitely far away have a potential energy of $1.52 \mathrm{eV}$ per pair, and two neutral atoms infinitely far away have no potential energy. Additional energy in the equilibrium system will cause a shift in the equilibrium distance. Note: $r$ in image is the same as $\mathrm{R}$ in this text 
Figure 10 - A representation of the various quantum numbers observed in ground state atoms. Alkali and alkaline earth metals have outer shell electrons in a $s$-state. Transition metals are in the process of filling their $d$-orbitals. Post-transition metals, metalloids, and nonmetals are depicted by filling their $p$-orbitals. The materials in particular interest for many phosphors

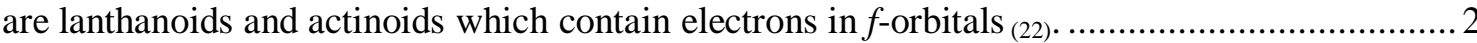

Figure $11-$ An $\varepsilon$ versus $k$ diagram displaying the quantum energy states which an electron may fill. Vertical lines represent electronic transitions where all changes in energy occur with adjustments in electron states. Vibrational energy transitions are depicted by changes in momentum (horizontal motion) and involve energy dissipation by means of lattice vibrations. The combination of both is referred to as a vibronic transition

Figure 12 - The absorption and emission spectrum of YAG:Ce provided by PhosphorTech. The various widths and heights of theses spectrums indicate the interaction between the host lattice and dopant $(25)$. 28

Figure 13 - This thesis investigates the feasibility of mimicking the Kelvin scale between 2500 and 7500 . The two temperatures on the chromaticity diagram represent the color that would be visible if an object (such as a tungsten filament in an incandescent bulb or steel in a mill) were heated up to those temperatures. The black body temperature curve extends from 1000 Kelvin (Orange) to 25000 Kelvin (Blue).

Figure 14 - Each set up is mounted on top of a Thorlabs optics table. On the left hand side of the image sits a beam splitter positioned to combine light from both Thorlabs LEDs and direct it into the center of the integrating sphere. The center of the images displays Cree LEDs mounted on linear stages where they are capable of moving under an optics tube.

Figure 15 - In the center of the images sits a computer monitor and Thorlabs power meter atop an Agilent DAQ. To their left sits two Thorlabs power supplies atop an Avantes spectrometer. All that equipment is positioned on a Newport linear motion controller which is surrounded by an Ocean Optics spectrometer and two Elenco power supplies. A National Instruments DAQ rests on top of the Elenco Power supplies

Figure 16 - A one-inch diameter aluminum substrate with a single rail down the diameter to allow for two LEDs to be mounted. Wire bonds extended from the top of each LED die to the corresponding PCB board (green rectangles). The flat portion on the aluminum substrate sides enables power wires to be routed without interfering with one-inch diameter optics tubes.

Figure 17 - An image of the actual set up ready for testing. Positive (red) and negative (black) wires come from the corresponding Elenco power supplies. A thermocouple (lowermost wire) monitors the aluminum substrate temperature during testing. 
Figure 18 - This Solidworks image displays two separate components. The top piece remains stationary during testing and houses the lenses and neutral density filters. The bottom piece houses the LEDs and sits atop linear stages that move according to the specified program.

Figure 19 - The benefit of this set up is that both the light sources emerge at one end of the beamsplitter, each at 50\% intensity, and interact with a phosphor substrate with minimal spatial displacement apparent between the two light sources. The integrating sphere collects all the light over a given area and directs it along a fiber optic cable, further reducing spatial displacement effects.

Figure 20 - Program menu enabling users to input numerics and select options necessary for testing. The majority of tests were conducted using the region titled 'LED Mapping' to characterize light emitted from the phosphor/PDMS substrates.

Figure 21 - Drawing depicting transparent PDMS easily being removed from a silicon wafer following a microfluidic processing technique. The silicon wafer contains a pattern for microfluidics and the PDMS mold contains the inverse of that pattern $(27)$.

Figure 22 - The arrangement of atoms within the YAG crystal structure. Each unit cell is comprised of a combination of a dodecahedrally coordinated yttrium atom (A), a tetrahedrally coordinated aluminum atom (B), and an octahedrally coordinated aluminum atom (C). Largest spheres (white) represent oxygen atoms, smallest spheres (light gray) represent aluminum atoms, and medium sized spheres (dark gray) represent yttrium atoms ${ }_{(29)}$.

Figure 23 - Four variables, three of which were tested with a 457nm Thorlabs LED held at constant amperage, that influence the observed color of light emitted from an LED.

Figure 24 - Displaying the total intensity of the visible spectrum at a given location over the cross-section of various thicknesses of $3.8 \mathrm{wt} \%$ phosphor/PDMS substrates passing directly over the LED die.

Figure 25 - Intensity comparisons between the six different, $2.5 \mathrm{~mm}$ thick, phosphor wt $\%$ samples. Intensity directly above the LED is substantially lower when phosphor is used due to its ability to diffuse light.

Figure 26 - The height measurements shown in this graph are measured from the top of the epoxy covering the 16 LED dies to the bottom of the phosphor substrate. Each height measurement was made with the $1.79 \mathrm{~mm}$ thick, $3.8 \mathrm{wt} \%$ sample. 
Figure 27 - The various colors created while looking directly above the LED die using different thickness phosphor/PDMS substrates. Height was maintained at $0 \mathrm{~mm}$ (contact with the LED's epoxy) and each sample has a phosphor concentration of $3.8 \mathrm{wt} \%$.

Figure 28 - Chromaticity values at a single point directly above the LED die. The 3.4 wt\% sample is still too blue considering the values of white light pursued in this project. All experiments were conducted using $2.5 \mathrm{~mm}$ thick samples and a height of $0 \mathrm{~mm}$.

Figure 29 - Height tests had a minimal effect on chromaticity values. There is a trend of decreasing chromaticity values with increasing height but it is negligible in comparison to the variance we see in thickness and concentration tests. All height tests were conducted with the $2.5 \mathrm{~mm}$ thick, $3.8 \mathrm{wt} \%$ sample.

Figure 30 - A graph displaying the color seen when looking at the $2.5 \mathrm{~mm}$ thick, one inch diameter, and $3.0 \mathrm{wt} \%$ phosphor/PDMS sample illuminated with the blue Thorlabs LED. While this graph displays color it does not relay intensity information to display brightness.

Figure 31 - A cross section of $\mathrm{x}$ - chromaticity values indicating the uniformity of color. A minimal change in chromaticity value indicates a more constant color over the cross section of the substrate. Each thickness experiment was conducted using $3.8 \mathrm{wt} \%$ phosphor in PDMS at a height of $0 \mathrm{~mm}$.

Figure 32 - This graph displays the $\mathrm{y}$ - chromaticity values with the same data used to produce Figure 31. Y - chromaticity values are more influenced by changes in phosphor concentration and thickness than $\mathrm{x}$ - chromaticity values. Each thickness experiment was conducted using $3.8 \mathrm{wt} \%$ phosphor in PDMS at a height of $0 \mathrm{~mm}$.

Figure 33 - Using chromaticity values obtained in a small region directly above the LED die during thickness testing of $3.8 \mathrm{wt} \%$ samples, a linear equation was fit to the points to display a slope of 0.0587 ( $\mathrm{x}$-chromaticity value $/ \mathrm{mm}$ ).

Figure 34 - Using chromaticity values obtained from radii not directly above the LED die during thickness testing of $3.8 \mathrm{wt} \%$ samples, a parabolic equation was fit to the $\mathrm{y}-$ chromaticity points.

Figure 35 - A 3 dimensional plot color coded to best relay information regarding the actual color of the observed chromaticity values. The plateaus at the bottom and top of the plot are regions which are not valid chromaticity values and therefore are points where the equations fail. This specific plot compares $\mathrm{x}$ - chromaticity effects of thickness and concentration over a 3 to $6 \mathrm{~mm}$ radial distance from the LED. 
Figure 36 - Absorption and fluorescent behavior of the specific $\mathrm{ZnS}$ :Se phosphor used. Absorption shows the ability for $\mathrm{ZnS}: \mathrm{Se}$ to absorb a given wavelength and the Emission displays the result of the selenium dopant to produce certain wavelengths ${ }_{(30)}$.

Figure 37 - A SEM image of phosphor particles in low vacuum. Mean particle diameter is stated to be 10 microns by PhosphorTech.

Figure 38 - The basic representation of PDMS backbone. It contains trimethyl groups on the ends of the chain and repeating units of ' $n$ ' amount of the monomer boxed in the center ${ }_{(32)}$

Figure 39 - Vinyl group found in PDMS at sections which have not been cross-linked. The carbon-to-carbon double bond is the key to a vinyl group.

Figure 40 - Silane group found within the agent which is necessary for a bonding site to the PDMS polymer backbone.

Figure 41 - Left: Simple representation of the $90 \mathrm{~mm}$ diameter by $2.5 \mathrm{~mm}$ height cast of PDMS mixed with the red ZnS:Se phosphor prior to curing. Right: Cured PDMS without any phosphor particles and a concentrated $\mathrm{ZnS}$ :Se layer at the bottom of mold.....

Figure 42 - The catalyst molecule which contains platinum, essential for inducing crosslinking $(37)$

Figure 43 - Left: Part of the polymer backbone which contains the vinyl group. Middle: Crosslinker molecule containing the hydrosilyl group. Right: With the addition of platinum, a cross-linked PDMS forming a solid structure.

Figure 44 - Representation of what is hypothesized to be occuring during the cure of PDMS and ZnS:Se. Left: A mold containing uniformly mixed phosphor in PDMS. Right: Given time to settle, $\mathrm{ZnS}$ :Se-free-PDMS at the top is allowed to cure.

Figure 45 - Left: A duplicate image found on the right hand side of Figure 44. Right: With additional time, all of the $\mathrm{ZnS}$ :Se settles out and PDMS is allowed to cure in its absence. What is left behind is a fully cured PDMS sample and ZnS:Se particles at the bottom in a mixture of uncured PDMS.

Figure 46 - Any color between the chromaticity value of the yellow phosphor and the chromaticity value of the blue LED can be obtained by changing intensities of one or the other. This was completed in a previous study by adjusting the concentration of the yellow phosphor which inherently changed its intensity while the blue LED was held at maximum current. 
Figure 47 - In between the dashed red and blue lines are all the color values possible using YAG:Ce, ZnS:Se, and a $457 \mathrm{~nm}$ blue LED.

Figure 48 - One experiment indicating ZnS:Se and YAG:Ce behavior when simultaneously exposed to blue and UV LEDs. 'Inverted' indicates the color values observed when the thin layer of $\mathrm{ZnS}: \mathrm{Se}$ was toward the integrating sphere as opposed to towards the light source. The lines represent all the color values possible by changing amperage to their corresponding LED.

Figure 49 - The simplest method to approximate the Kelvin temperatures between 2500 and 7500 is to adjust the intensities of color sources with chromaticity values along the linear approximation curve outside of the 2500 to 7500 interval.

Figure 50 - A linear approximation of the 2500 to 7500 Kelvin temperatures with one light source producing a color value of $488 \mathrm{~nm}$.

Figure 51 - One half of the complex crystal structure of BAM:Eu. This half shows one of the possible positions for europium to reside in ${ }_{(43)}$.

Figure 52 - Absorption and Emission spectrum of BAM:Eu used in this study (44)

Figure 53 - One of the many configurations of BAM:Eu phosphor in PDMS positioned near PDMS with YAG:Ce and ZnS:Se phosphors. This design requires light to come from the bottom so that an observer (above the phosphors) would not see any stimulation of YAG:Ce or $\mathrm{ZnS}: \mathrm{Se}$ caused by excited BAM:Eu.

Figure 54 - BAM:Eu produces chromaticity values in the cyan region of this $1931 \mathrm{CIE}$ diagram. When placing BAM:Eu on the LED side of the YAG:Ce and ZnS:Se PDMS substrate, UV excitation causes a blue emission from BAM:Eu which then induces a yellow emission from the YAG:Ce. The result is a more yellow color value. In the opposite orientation, stimulated BAM:Eu would cause a blue-shift but not substantial enough for a multi-Kelvin LED design.

Figure 55 - Actual data from testing completed with a $457 \mathrm{~nm}$ LED and specific concentrations of YAG:Ce and ZnS:Se phosphors illuminated with a $457 \mathrm{~nm}$ LED while a separate $479 \mathrm{~nm}$ blue LED provided additional light to increase the Kelvin temperature. A closer approximation would be possible by adjusting the YAG:Ce and ZnS:Se concentration for that specific $479 \mathrm{~nm}$ blue LED as opposed to the $488 \mathrm{~nm}$ phosphor it was designed for.

Figure 56 - One possible set up which would yield a white light source that could create Kelvin values between 2500 and 7500 . The $457 \mathrm{~nm}$ blue LED would stimulate a phosphor combination to obtain a color value near 2500 Kelvin on the linear approximation curve 
while the $484 \mathrm{~nm}$ blue LED provides the necessary intensity to adjust that color value up to 7500 Kelvin.

Figure 57 - Displaying the optimum linear approximation of the Kelvin scale between 2500 and $7500 \mathrm{~K}$ based off of the chromaticity value for a 479 peak-wavelength Thorlabs LED

Figure 58 - A significant decrease in photoluminescent intensity over a 900 second (15 minute) interval. The intensity of the $457 \mathrm{~nm}$ blue Thorlabs LED remains essentially unchanged throughout this time period.

Figure 59 - The peak intensity of the photoluminescent region, comprised of YAG:Ce and $\mathrm{ZnS}: \mathrm{Se}$, throughout a 900 second interval. The decay appears to reach a plateau around 10 minutes of exposure to the $457 \mathrm{~nm}$ Thorlabs LED at $670 \mathrm{~mA}$.

Figure 60 - Due to the degradation of $\mathrm{ZnS}:$ Se phosphor the closest approximation to the specific intervals desired on the Kelvin scale deviated from the calculated fit. Prior to prolonged blue light exposure the chromaticity values of this substrate were more yellow than $2500 \mathrm{~K}$.

Figure 61 - Data from a previous study indicating what thickness of a phosphor substrate is required to produce the same chromaticity values. A specific Gaussian distribution would be required for a given LED configuration.

Figure 62 - SolidWorks depiction of a one-inch diameter disk with a peak thickness of 4.74 $\mathrm{mm}$ and a minimal thickness of $1.71 \mathrm{~mm}$.

Figure 63 - A model of the casting tray created by Z-Corp rapid prototyping.

Figure 64 - Measured chromaticity values on a Gaussian surface at various radii from the LED center.

Figure 65 - Above a flat phosphor/PDMS surface chromaticity values changed dramatically with changes in radii from the LED center. This translates to a poor white LED illumination source.

Figure 66 - The chromaticity deviation at various radii was large in the constant thickness sample as expected based on previous tests. The Gaussian profile sample had much less of a deviation over the same radial distance.

Figure 67 - Intensity above the Gaussian lens still displays more counts directly above the LED than at the outer edges but the design did improve over a constant thickness lens. 110

$$
\text { xix |P a g e }
$$


Figure 68 - In addition to the large intensity gradient with radii, blue (380-510 nm) wavelengths are shown to dominate the spectrum at lower radii and decrease below photoluminescent wavelengths $(510-770 \mathrm{~nm})$ which is the cause for the large color gradient observed in constant thickness phosphor/PDMS lenses.

Figure 69 - To produce warm light a spectrum dominated by YAG:Ce and ZnS:Se luminescence is required.

Figure 70 - With the 479 nm LED, 457 nm LED, YAG:Ce, and ZnS:Se equal peak intensities (brightest the bulb would be) is at 2900 Kelvin and would shift depending upon the LED wavelengths and phosphor concentration.

Figure 71 - To produce cool-white light the spectrum must be dominated with the $479 \mathrm{~nm}$ LED light and a significantly less phosphor contribution. This curve would be the dimmest of all the colors on the Kelvin approximation curve.

Figure 72 - Normalized intensity of light at a given Kelvin temperature. The red curve is changed while holding the $457 \mathrm{~nm}$ LED/YAG:Ce/ZnS:Se combination intensity constant. The blue curve was the result while holding the $479 \mathrm{~nm}$ LED constant.

Figure 73 - Cartoon depiction of a LED lamp with $457 \mathrm{~nm}$ blue LEDs coated with a YAG:Ce and $\mathrm{ZnS}$ :Se phosphor combination dispersed periodically along with $484 \mathrm{~nm}$ blue LEDs.

Figure 74 - In this kind of apparatus a waveguide would need to be deposited directly over the LED. Light would be directed with minimal loss or dispersion through the waveguide and in the phosphor substrate.

Figure 75 - The mask was designed based on size and spacing of numeric oriented pixels produced by General LED for various applications. The final six digit configuration is designed to overlay on top of the LED pixels to change blue LEDs into an apparent uniform white color.

Figure 76 - Top and side view of a Gaussian-like profile created above each of the seven pixels which comprise a single digital number eight.

Figure 77 - Phosphor datasheet of the specific YAG:Ce phosphor purchased from PhosphorTech. Absorption and Emission profiles are utilized to design a white LED, shown in the profile directly underneath.

Figure 78 - All the possible color values obtainable with a given blue LED using the specific YAG:Ce phosphor utilized throughout all of the experiments. 
Figure 79 - Intensity comparisons between the five different, $2.5 \mathrm{~mm}$ thick, phosphor wt\% samples. Increasing the amount of phosphor above the LED decreases blue light intensity emitted orthogonal the substrate surface. Intensity profiles here reflect the amount of blue light passing through the substrate.

Figure 80 - Intensity comparisons between the five different, $2.5 \mathrm{~mm}$ thick, phosphor wt $\%$ samples. Increasing the amount of phosphor above the LED increases yellow light intensity emitted orthogonal the substrate surface. Intensity profiles here reflect the amount of yellow light emitted by the phosphor.

Figure 81 - Intensity comparisons between the five different, $2.5 \mathrm{~mm}$ thick, phosphor wt $\%$ samples. Increasing the amount of phosphor above the LED increases the overall visible light intensity emitted orthogonal the substrate surface. Intensity profiles here reflect the entire visible spectrums intensity.

Figure 82 - A cross section of $\mathrm{x}$ - chromaticity values indicating the uniformity of color. A minimal change in chromaticity value indicates a more constant color over the cross section of the substrate. Each concentration experiment was conducted using $2.5 \mathrm{~mm}$ thick substrate at a height of $0 \mathrm{~mm}$.

Figure 83 - This graph displays the y - chromaticity values with the same data used to produce Figure 81 . Y - chromaticity values are more influenced by changes in phosphor concentration and thickness than $\mathrm{x}$ - chromaticity values. Each concentration experiment was conducted using $2.5 \mathrm{~mm}$ thick substrate at a height of $0 \mathrm{~mm}$.

Figure 84 - A cross section of $\mathrm{x}$ - chromaticity values indicating the uniformity of color. Adjusting the height has a minimal effect on color values. Each height experiment was conducted using a $1.79 \mathrm{~mm}$ thick, $3.8 \mathrm{wt} \%$ substrate.

Figure 85 - A cross section of y - chromaticity values indicating the uniformity of color. Adjusting the height has a minimal effect on color values. Each height experiment was conducted using a $1.79 \mathrm{~mm}$ thick, $3.8 \mathrm{wt} \%$ substrate.

Figure 86 - A 3 dimensional plot color coded to best relay information regarding the actual color of the observed chromaticity values. The plateaus at the bottom and top of the plot are regions which are not valid chromaticity values and therefore are points where the equations fail. This specific plot compares $\mathrm{x}$ - chromaticity effects of thickness and concentration in a $1 \mathrm{~mm}$ radial distance from the $\mathrm{LED}$.

Figure 87 - A 3 dimensional plot color coded to best relay information regarding the actual color of the observed chromaticity values. The plateaus at the bottom and top of the plot are regions which are not valid chromaticity values and therefore are points where the equations 
fail. This specific plot compares y - chromaticity effects of thickness and concentration in a $1 \mathrm{~mm}$ radial distance from the LED.

Figure 88 - A 3 dimensional plot color coded to best relay information regarding the actual color of the observed chromaticity values. The plateaus at the bottom and top of the plot are regions which are not valid chromaticity values and therefore are points where the equations fail. This specific plot compares y - chromaticity effects of thickness and concentration over a 3 to $6 \mathrm{~mm}$ radial distance from the LED.

Figure 89 - A 3 dimensional plot color coded to best relay information regarding the actual color of the observed chromaticity values. The plateaus at the bottom and top of the plot are regions which are not valid chromaticity values and therefore are points where the equations fail. This specific plot compares $\mathrm{x}$ - chromaticity effects of height and thickness in a $1 \mathrm{~mm}$ radial distance from the LED.

Figure 90 - A 3 dimensional plot color coded to best relay information regarding the actual color of the observed chromaticity values. The plateaus at the bottom and top of the plot are regions which are not valid chromaticity values and therefore are points where the equations fail. This specific plot compares $y$ - chromaticity effects of height and thickness in a $1 \mathrm{~mm}$ radial distance from the LED.

Figure 91 - A 3 dimensional plot color coded to best relay information regarding the actual color of the observed chromaticity values. The plateaus at the bottom and top of the plot are regions which are not valid chromaticity values and therefore are points where the equations fail. This specific plot compares $\mathrm{x}$ - chromaticity effects of height and thickness over a 3 to 6 $\mathrm{mm}$ radial distance from the LED.

Figure 92 - A 3 dimensional plot color coded to best relay information regarding the actual color of the observed chromaticity values. The plateaus at the bottom and top of the plot are regions which are not valid chromaticity values and therefore are points where the equations fail. This specific plot compares y - chromaticity effects of height and thickness over a 3 to 6 $\mathrm{mm}$ radial distance from the LED.

Figure 93 - A 3 dimensional plot color coded to best relay information regarding the actual color of the observed chromaticity values. The plateaus at the bottom and top of the plot are regions which are not valid chromaticity values and therefore are points where the equations fail. This specific plot compares $\mathrm{x}$ - chromaticity effects of height and concentration in a 1 $\mathrm{mm}$ radial distance from the $\mathrm{LED}$.

Figure 94 - A 3 dimensional plot color coded to best relay information regarding the actual color of the observed chromaticity values. The plateaus at the bottom and top of the plot are regions which are not valid chromaticity values and therefore are points where the equations 
fail. This specific plot compares y - chromaticity effects of height and concentration in a 1 $\mathrm{mm}$ radial distance from the $\mathrm{LED}$

Figure 95 - A 3 dimensional plot color coded to best relay information regarding the actual color of the observed chromaticity values. The plateaus at the bottom and top of the plot are regions which are not valid chromaticity values and therefore are points where the equations fail. This specific plot compares $\mathrm{x}$ - chromaticity effects of height and concentration over a 3 to $6 \mathrm{~mm}$ radial distance from the LED.

Figure 96 - A 3 dimensional plot color coded to best relay information regarding the actual color of the observed chromaticity values. The plateaus at the bottom and top of the plot are regions which are not valid chromaticity values and therefore are points where the equations fail. This specific plot compares y - chromaticity effects of height and concentration over a 3 to $6 \mathrm{~mm}$ radial distance from the LED.

Figure 97 - The change in chromaticity values with radial distance decreases compared to the $4.49 \mathrm{~W}$ tests, however variation for specific radii is greater and chromaticity noise at $10 \mathrm{~mm}$ is extremely large due to insufficient illumination.

Figure 98 - At only $1.51 \mathrm{~W}$ the luminous emittance is greatly reduced but uniformity is increased over the two other higher power tests for the interval between 0 and 9 mm radii 


\section{CHAPTER I: BACKGROUND}

\section{Overview}

In this thesis we examine the feasibility of developing a white light source capable of producing colors between 2500 and 7500 Kelvin $(\mathrm{K})$ by simply adjusting amperage to a blue and ultraviolet (UV) light emitting diode (LED). The purpose of this lighting source is to better replicate daylight inside a building at a given time of day. Where $2500 \mathrm{~K}$ replicates warm-white light similar to sunrise and sunset, $7500 \mathrm{~K}$ approximates coldwhite light seen with a clear blue sky, and Kelvin values in between those extremes mimics other white colors visible throughout a typical day. This study analyzes the proposed light source using a $385 \mathrm{~nm}$ UV LED, a $457 \mathrm{~nm}$ blue LED, a $479 \mathrm{~nm}$ blue LED, a $562 \mathrm{~nm}$ peak cerium doped yttrium aluminum garnet (YAG:Ce) phosphor, a $647 \mathrm{~nm}$ peak selenium doped zinc sulfide (ZnS:Se) phosphor, and a $488 \mathrm{~nm}$ peak europium doped barium magnesium aluminate (BAM:Eu) phosphor. The above luminescent sources are listed in Table I.

Table I - Important LEDs and Phosphors

\begin{tabular}{|l|c|}
\hline Luminescent Source & $\begin{array}{l}\text { Emitted Peak } \\
\text { Wavelength (nm) }\end{array}$ \\
\hline Thorlabs Ultraviolet LED & 385 \\
Thorlabs Blue LED (A) & 457 \\
Thorlabs Blue LED (B) & 479 \\
YAG:Ce & 562 \\
ZnS:Se & 647 \\
BAM:Eu & 488 \\
\hline
\end{tabular}


This method of creating a white LED is known as a phosphor-converted LED (pcLED). In the current market this type of white light source most likely incorporates a series of blue-emitting LED dies with a yellow phosphor (such as YAG:Ce) in such a combination to produce what appears to be white light.

Our initial approach to this study examined optical performance of yellow-emitting phosphor (YAG:Ce) positioned at specific distances above a blue LED using polydimethylsiloxane (PDMS) as a substrate. PDMS, which is simple to manufacture, provided the optically transparent media necessary for an illumination source while also preventing physical interaction between the phosphor and the die. The study revealed how phosphor concentration within PDMS, PDMS thickness, and PDMS distance from the LED die affected light intensity and color values (determined quantitatively by utilizing $1931 \mathrm{CIE} 2^{\circ}$ Standard Observer). These results provided the reasoning for which a red light emitting phosphor was also necessary for this multi-Kelvin illumination source. Red-emitting phosphor (ZnS:Se) and YAG:Ce dispersed throughout PDMS and stimulated with a blue LED had the capabilities of obtaining all desired Kelvin values of white light but only with changes in concentration. Even stimulating ZnS:Se separate with a UV LED did not provide the ability to change the color value of the set up to the degree required. The final design incorporated a combination of $\mathrm{ZnS}: \mathrm{Se}$ and YAG:Ce stimulated with a blue LED to obtain a 2500 Kelvin value. A separate blue LED provides the means to obtain 7500 Kelvin light and the other color values in between by adjusting the amperages of both LEDs. In addition to investigating the feasibility of obtaining the Kelvin values from 2500 to 7500, this thesis also examined ZnS:Se's 
inability to cure in PDMS and a method to create a Gaussian lens shape to provide equal color values at all points above a LED source.

\section{Broader Impact}

High-powered white LEDs have the potential to replace most forms of commercial light sources. They are capable of producing visible light in greater efficiencies than incandescent and fluorescent style light sources. Philips Lumileds Lighting Company claimed the most efficient high-powered white LED at 115 luminous per watt in 2008 as compared to standard incandescence bulbs at 15 lumens per watt and fluorescent bulbs

near 100 lumens per watt ${ }_{(1)}$. LEDs are best suited for task specific lighting due to the collimated light emitted from the die. Task specific lighting includes standard household lighting applications, flashlights, streetlights, vehicle lighting, architectural lighting, backlighting for LCD televisions and lightweight laptop displays, stage lights, and medical lighting where infrared radiation and high temperatures are not desired $(2)$. Solid state lighting technology is very resistant to vibrations, as compared to incandescence, and does not contain harmful chemicals such as mercury which is found in fluorescent devices. The Energy Independence and Security Act of 2007 require that generalpurpose light bulbs become 30\% more efficient starting with the 100 Watt bulbs in 2012 and ending with 40 Watt bulbs in $2014_{(3)}$. This will essentially provide consumers with the option of selecting a compact fluorescent (CFL) or a LED for white light illumination. The long life and durability of solid state lighting, more environmentally friendly materials, and much improved energy efficiency enable white LEDs to positively impact the world. 


\section{Stakeholder's Needs}

Examining the feasibility of a multi-Kelvin temperature white LED is a funded project by General LED headquartered in Texas. General LED is a 3 year old company but has 6 years of field success obtained from AgiLight Incorporated, their wholly-owned subsidiary. This project is of direct interest to the members of the Lighting division of General LED. It was the cooperation with them, especially Bill Ratcliffe (Founder and CTO) that enabled this study to continue.

\section{Beneficial Health Aspects}

A multi-Kelvin temperature white LED is beneficial to human health since it can better replicate the circadian cycle. The circadian rhythm is a natural cycle (approximately 24 hours) in living entities. Human activities such as mental awareness, mood, and the sleep/wake cycle are regulated by environmental stimuli throughout the day. Proper circadian regulation requires a light source which not only provides short wavelength radiation (blue light) but also the proper intensity, timing, and duration of exposure. This stems from the fact that daylight does not have a fixed spectrum. The color of daylight is dependent upon latitude, time, season, cloud cover, air pollution, and ground reflectance. In general people prefer lighting from windows and skylights rather than manufactured sources. This may stem from the cyclic nature and color adjustments throughout the day which conventional lighting fails to replicate. Slight positive shifts in a given persons

mood due to a more natural appearing environment may increase productivity (4). A light source with an ability to display objects with the color they would appear to be in 
sunlight and one which will adjust its color based on the color of outdoor light would address these human health benefits.

\section{Constraints Applied to this Study}

To better approximate light observed throughout an average day, a white light source must be capable of adjusting its Kelvin value throughout the day (Figure 1).

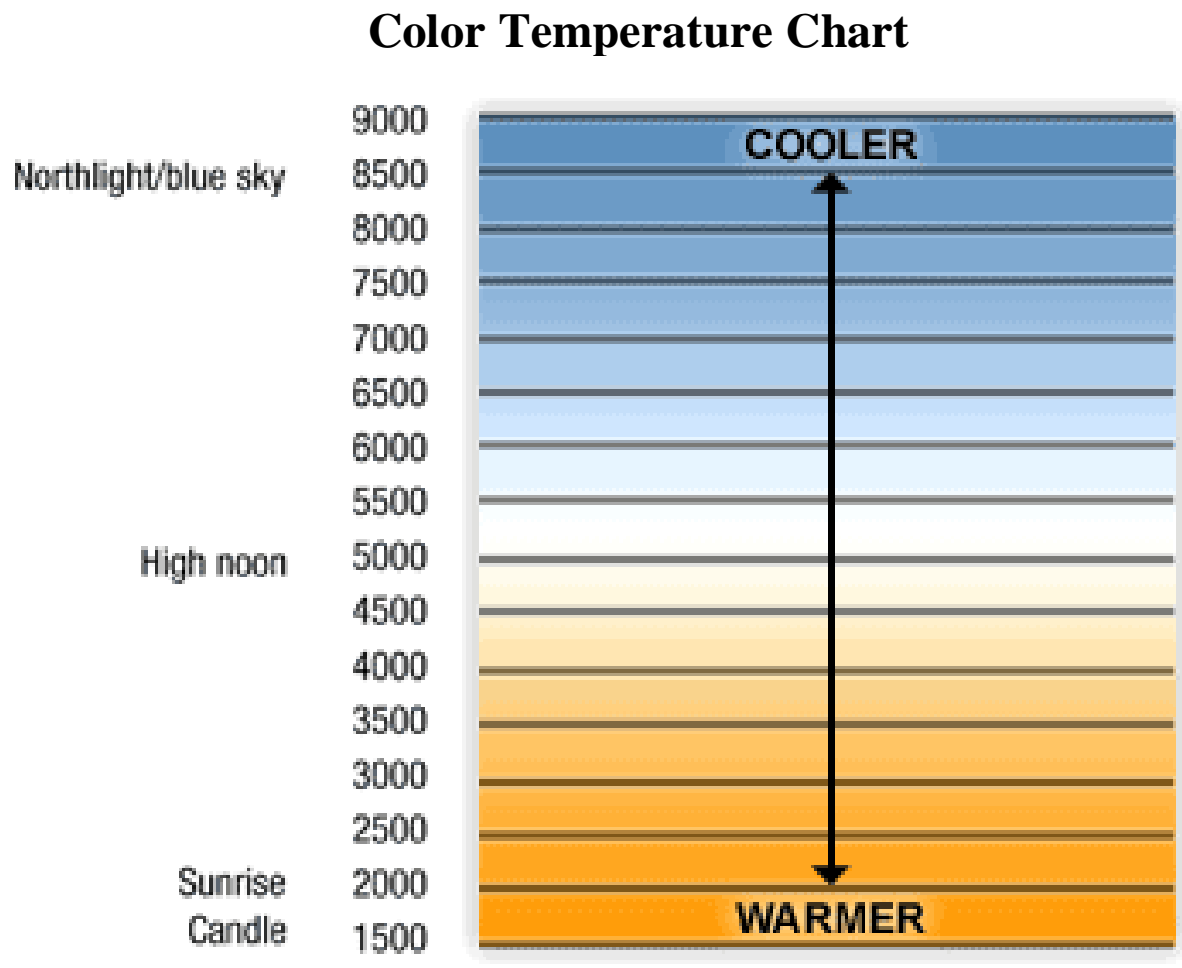

Figure 1 - Depicts the color of light observed for a given Kelvin temperature displayed on the vertical $\operatorname{axis}_{(5)}$.

Kelvin values observed in daylight are not specific at a given time, given day, or even given location. However, this study will assume that warm-light observed at sunset and sunrise is 3200 Kelvin, overcast is 7000 Kelvin, and light seen in the middle of the day is approximately 5500 Kelvin. 
The desired LED configuration is to use multiple blue and UV LEDs in combination with a single phosphor coating to create a white LED which produces all Kelvin values between 2500 and 7500 (Figure 2).

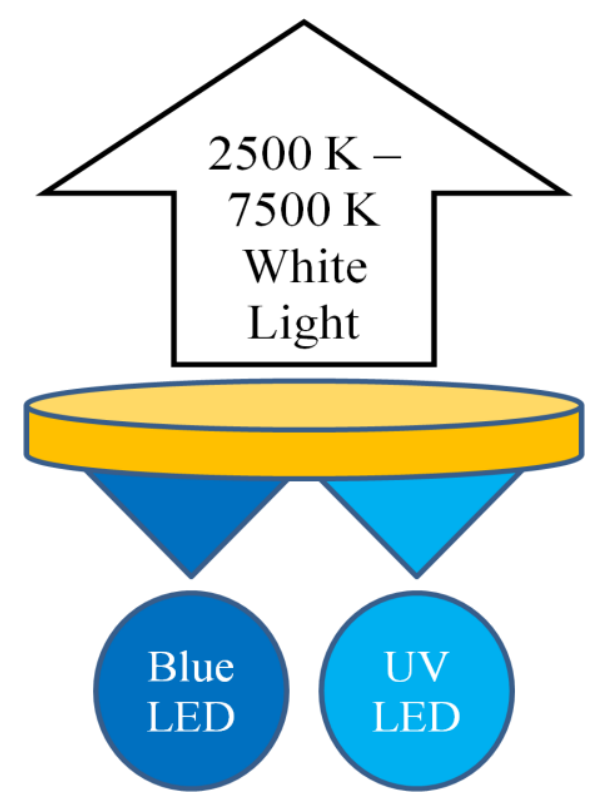

Figure 2 - A representation of blue and UV LED sources both illuminating the same phosphor substrate to deliver the desired white light to an observer. The goal is to be able to adjust amperage to both LEDs in such a way which will result in a 5000 Kelvin change in color values to an observer of the phosphor substrate.

In this configuration one of the LEDs will stimulate some combination of phosphors within the substrate to result in a color of white light on the Kelvin scale. The additional LED will provide extra stimuli to the same phosphors and/or phosphors within the substrate to change the Kelvin temperature of the observed light. Unfortunately this configuration had many downsides, including low luminous emittance, which resulted in the final recommendation to use a blue LED and a phosphor-converted LED (pcLED). 
Polydimethylsiloxane (PDMS) has been utilized for this study as a substrate for the various phosphors necessary to create white light. PDMS is relatively simple to cast in the desired shape required for different tests and it is transparent to visible (380 to 770 $\mathrm{nm}$ ) and ultraviolet (300 to $380 \mathrm{~nm}$ ) light. 


\section{CHAPTER II: INTRODUCTION TO PHOSPHORS}

\section{Electromagnetic Spectrum}

Results throughout this document pertain to values of intensity for a corresponding wavelength on the electromagnetic spectrum. The spectrum may be described using energy of a photon (E), frequency $(f)$, or wavelength $(\lambda)$ as they are related by Planks Constant $(h)$ and the speed of light in a vacuum $(c)$ with the following equation.

$$
E=h f=\frac{h c}{\lambda} \quad \text { Equation } 1
$$

The focus of this study is on the visible and ultraviolet (specifically UVA) portion of the spectrum spanning from 315 to $770 \mathrm{~nm}$. When describing the visible spectrum, wavelength values will be reported where blue light can be thought of as 380 to $490 \mathrm{~nm}$, green light as 510 to $560 \mathrm{~nm}$, yellow as 570 to $590 \mathrm{~nm}$, and red as 620 to $770 \mathrm{~nm}$ along the electromagnetic spectrum. The remainder of the spectrum consists of radio waves, microwaves, infra-red radiation, x-rays, and gamma-rays from least energetic to most energetic (Figure 3). 


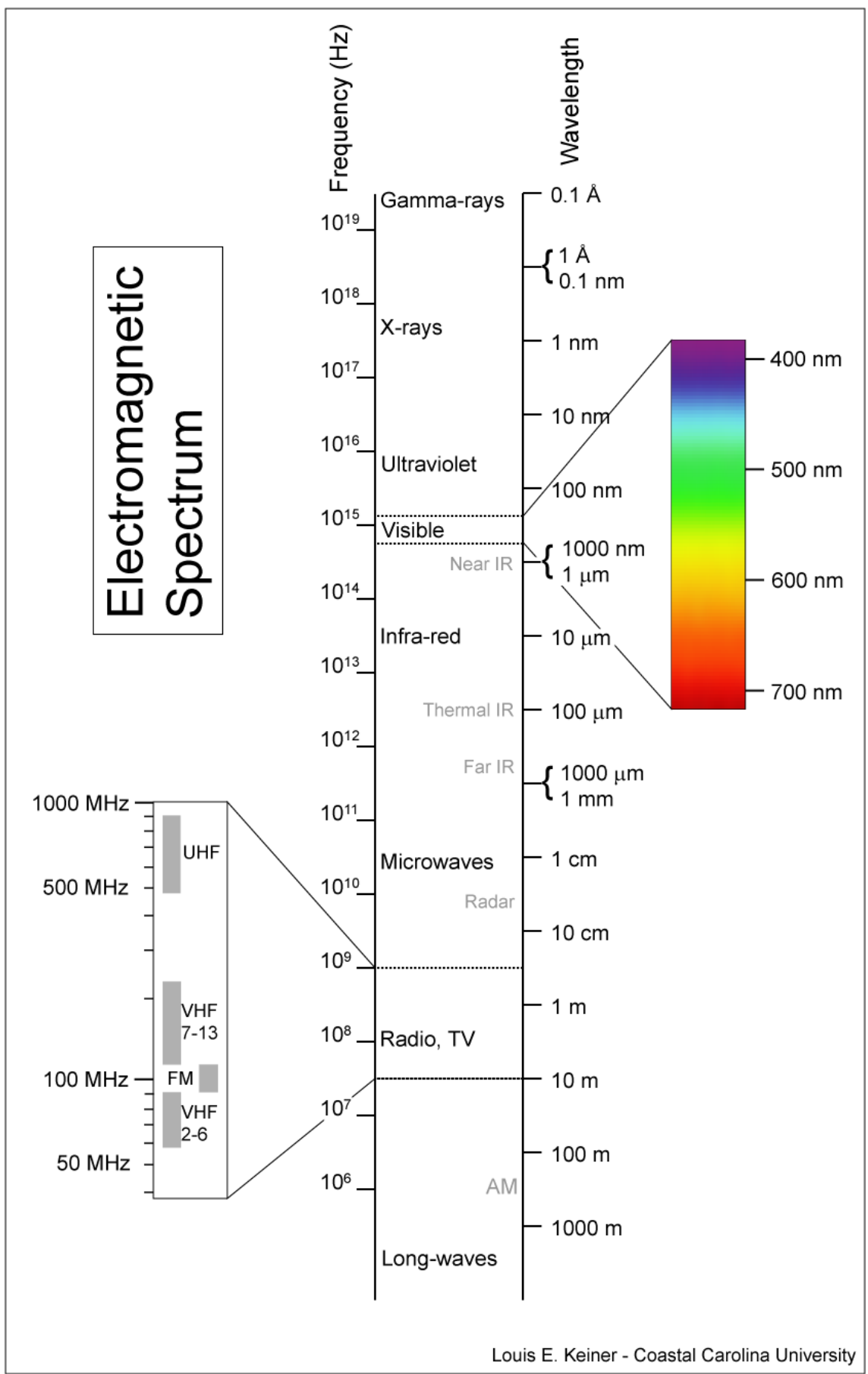

Figure 3 - A depiction of the electromagnetic spectrum from 0.1 angstroms to 1000 meters. A detailed view of the visible spectrum displays the colors observed at each wavelength from 380 to 770 $\mathbf{n m}_{(6)}$. 


\section{What are Phosphors}

A phosphor material is a substance comprised of multiple elements which are capable of absorbing photons or electrons and reemitting that energy in the form of photons. In the realm of this project, phosphors selected absorbed UV or blue color light and emitted blue, yellow, or red color light. For example, one phosphor material (YAG:Ce) absorbed blue light and converted that energy into yellow light emission (Figure 4).

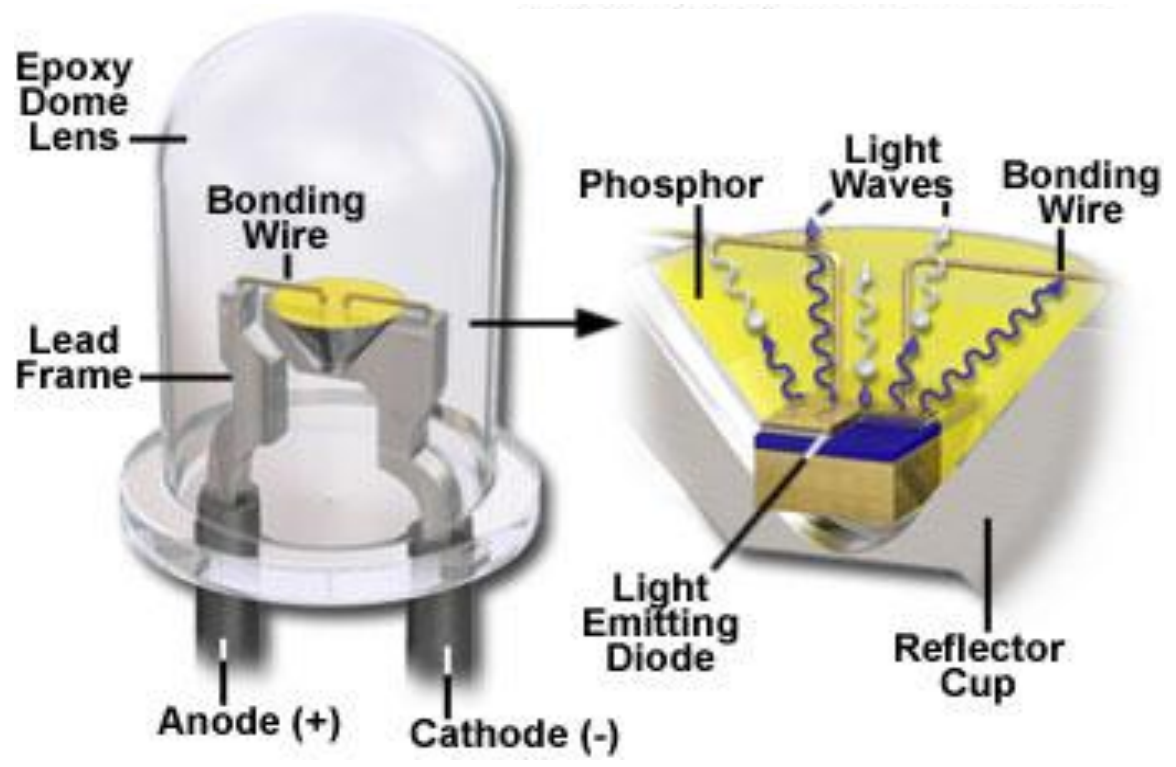

Figure 4 - The cross-sectional view of a low-power white LED. The LED is positioned at the bottom of the reflector cup and covered by yellow phosphor. Some of the blue light stimulates the phosphor to enable yellow photoluminescence. The combination of blue light, which did not interact with the phosphor, and yellow light emitted from that region appear white to the human eye ${ }_{(7)}$.

Phosphors should not be confused with the element, phosphorus, which exhibits a different method of light production known was chemiluminescence (light production due to a chemical reaction) (8). A phosphor material displays a photoluminescence property where, as stated earlier, electromagnetic radiation is absorbed and over a given time period (sometimes on the order of nanoseconds) a different energy photon is 
emitted. This is different from the process common in incandescent bulbs where a material, tungsten filament in most cases, is heated to temperatures which provide enough energy for emitted radiation to be in the visible spectrum. The method of heating to produce light comes close to approximating the black-body radiator color values which is why incandescent light bulbs have an inherently good color rendering index (this will be discussed in later sections of this document).

A photoluminescent material consists of a host lattice with small quantities of luminescent centers distributed throughout (9). Host lattice refers to the arrangement of atoms which compose a substance (Figure 5).

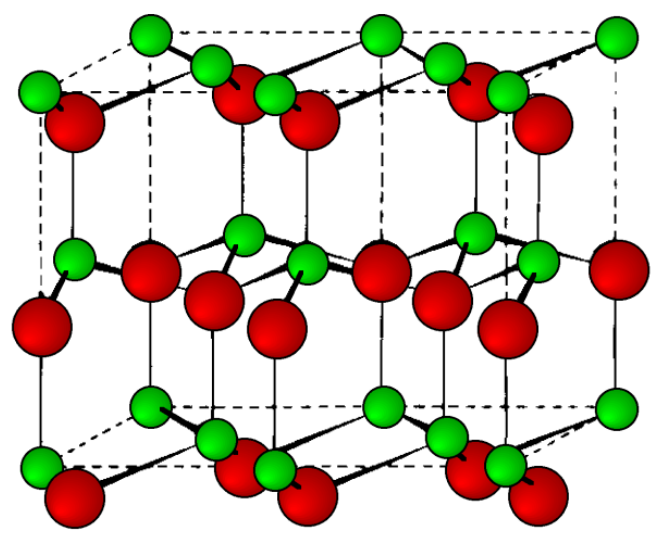

Figure 5 - A zinc sulfide ( $\mathrm{ZnS}$ ) unit cell (represented by dashed lines) of zinc (small/green) and sulfur (large/red) atoms arranged in what is known as the wurtzite lattice. These cubic cells are repetitive throughout the entire substance ${ }_{(10)}$.

Luminescent centers are essentially locations within the host lattice that enable photon production (refer to Chapter III for greater detail). These locations are typically created by vacancies, interstitials, substitutions, or antisite defects (Figure 6). 


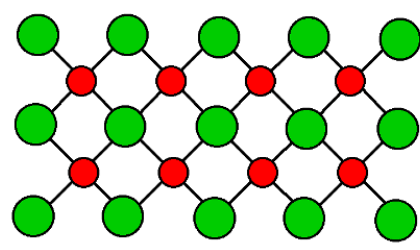

(a) perfect lattice

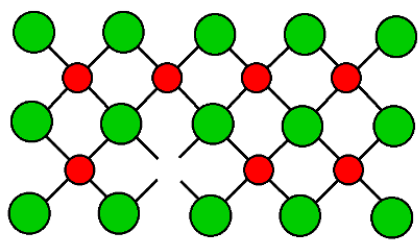

(c) cation vacancy

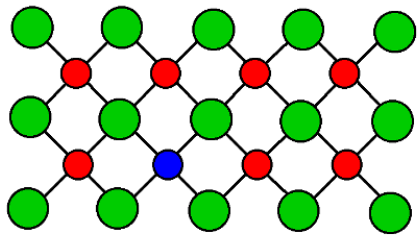

(e) substitution of cation

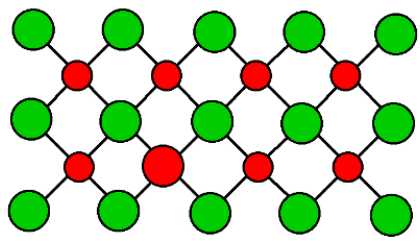

(g) $\mathrm{B}_{\mathrm{A}}$ antisite defect

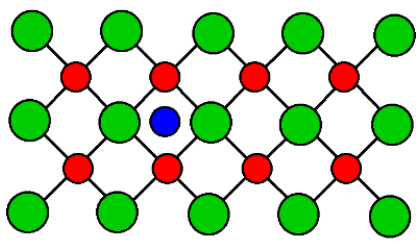

(b) interstitial impurity

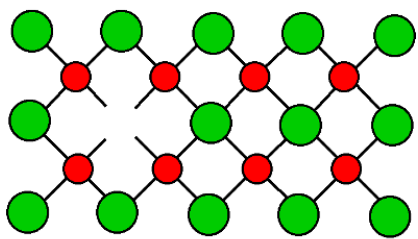

(d) anion vacancy

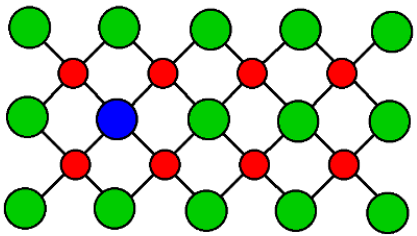

(f) substitution of anion

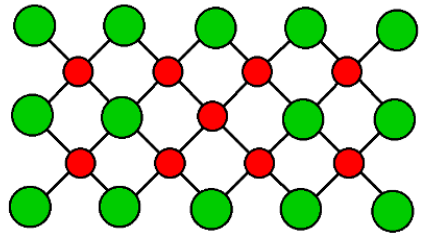

(h) $\mathrm{A}_{\mathrm{B}}$ antisite defect

Figure 6 - In semiconductor materials point defects, such as vacancies, interstitial impurities, or antisite defects, provide the luminescent centers necessary for light emission. In other inorganic phosphors, such as YAG:Ce or BAM:Eu, substitution atoms become the luminescent center ${ }_{(10)}$.

The host lattice will usually be an oxide (chemical compound with at least one oxygen element and one other element), nitride (generally a nitride ion in combination with a less electronegative ion), or oxynitrides (an element in combination with an oxygen and nitrogen atom). The luminescent center will typically consist of a transition metal or a rare earth element which substitutes for other elements in the host lattice ${ }_{(11)}$. 


\section{How are Phosphors Manufactured}

There are many ways to prepare phosphors due to the wide range of host lattice materials and trace elements used to create the luminescent centers. Due to the extensive use of YAG:Ce within this study, the various ways to create that particular phosphor are discussed below. A brief overview of the solid-state reaction, co-precipitation, sol-gel, and combustion methods for forming YAG:Ce are provided based on experiments conducted by Yuexiao Pan ${ }_{(12)}$. The solid-state reaction method includes milling a mixture of yttrium oxide $\left(\mathrm{Y}_{2} \mathrm{O}_{3}\right)$, cerium oxide $\left(\mathrm{CeO}_{2}\right)$, and aluminum hydroxide $\left(\mathrm{Al}(\mathrm{OH})_{3}\right)$ particles in a ratio of 2.88 parts yttrium, 5 parts aluminum, and 0.12 parts cerium (2.88Y:5Al:0.12Ce). Crystallization is achieved by placing the milled particles in a $1300^{\circ} \mathrm{C}$, carbon monoxide (CO) environment, furnace for 10 hours. For efficiency purposes, achieved by increasing the amount of crystallinity, the phosphor particles are fired under the same settings but this time at $1500^{\circ} \mathrm{C}$. A co-precipitation method includes a solution of the same molar concentration used above, added drop wise to ammonium bicarbonate $\left(\mathrm{NH}_{4} \mathrm{HCO}_{3}\right)$ forming precipitates. The solution is formed by dissolving $\mathrm{Y}_{2} \mathrm{O}_{3}$ in nitric acid $\left(\mathrm{HNO}_{3}\right), \mathrm{CeO}_{2}$ in $\mathrm{HNO}_{3}$, and aluminum nitrate $\left(\mathrm{Al}\left(\mathrm{NO}_{3}\right)_{3}\right)$ in distilled water. The solution is then filtered and rinsed with distilled water, dried at $100^{\circ} \mathrm{C}$ and placed in a $\mathrm{CO}$ atmosphere furnace at $1000^{\circ} \mathrm{C}$. The sol-gel method is a popular technique that also includes starting out with a solution of the same molar ratio used in the first two methods. This time the solution is added dropwise to citric acid $\left(\mathrm{C}_{6} \mathrm{H}_{8} \mathrm{O}_{7}\right)$ in ethylene glycol. $200^{\circ} \mathrm{C}$ heat is applied for a few hours followed by a $400^{\circ} \mathrm{C}$ firing when excess solvent is removed. The remaining precipitate is ground into a powder and fired at $1000^{\circ} \mathrm{C}$ in air (as opposed to $\mathrm{CO}$ as discussed before). The combustion method again uses the solution 
with the molar ratio of $2.88 \mathrm{Y}: 5 \mathrm{Al}: 0.12 \mathrm{Ce}$ but this time the solution is boiled. While boiling urea and solid yttrium oxide are added eventually creating white foam. Upon drying, the foam is milled and sintered at $1000^{\circ} \mathrm{C}$. All these methods produce nano-scale to micro-scale size particles which have the cerium atom dispersed throughout a garnet host lattice thus creating a luminescent center. The key with all these methods is that the amount of crystallization in the host lattice needs to be as large as possible to increase luminescence. This is accomplished with sintering at high temperatures to enable Ce ions to substitute into yttrium locations of the YAG:Ce lattice.

\section{Applications}

This particular study focuses on phosphor usage in white LEDs. However phosphors may be found in other types of illumination or display technology. For instance, most of the visible light from a fluorescent bulb comes from phosphors on the inner surface of the glass tube. The low pressure gas (mercury in conjunction with argon, xenon, neon, or krypton) inside the tube is excited due to an electrical current maintained over the length of the tube. The excited gas typically emits light in the ultraviolet spectrum which is converted by the phosphor to the visible spectrum for illumination. Glow-in-the-dark toys and face paints are possible because of phosphors. By dispersing certain phosphors into materials such as hard plastics, polymers, or even paints (make-up) specific colors of light may be provided long after (often several hours) their initial exposure to light. The reason some phosphors may continue to emit light long after their initial excitation will be discussed in greater detail in the next chapter. Cathode ray tubes (CRTs) are made possible due to phosphor coatings on the inside of the glass screen. CRTs consist of an 
electron source sealed in a vacuum tube, some electrode configuration to accelerate the electrons from the source to the screen, a series of coils to focus the electron beam, and phosphors which may be excited upon exposure to the electron beam. Early CRTs (black-and-white television) utilized a pair of phosphors to create a white light. More recent CRTs use three electron beams (one for each color) and a screen with red, green, and blue phosphors arranged periodically. In both designs specific locations of phosphors are excited, forming each pixel, and in combination with many pixels yields an image. Since these phosphors are excited by an electron beam, as opposed to photons, they exhibit what is known as electroluminescence ${ }_{(13)}$.

\section{Broad Spectrum Benefits}

In illumination applications it is beneficial to have a light source which contains a significant portion of all visible wavelengths, as the sunlight does, in order to enable objects to appear their true color. In display applications this is not of great concern because nearly every color may be obtained with a single wavelength of blue light, a single wavelength of green light, and a single wavelength of red light. However while a combination of three wavelengths is able to produce the majority of colors, it leaves out a large amount of wavelengths inherent to visible light. In illumination applications if a light source with a small quantity of wavelengths is incident upon an object the light reflected or transmitted through the object to an observer may appear different than it would under sunlight (Figure 7). 


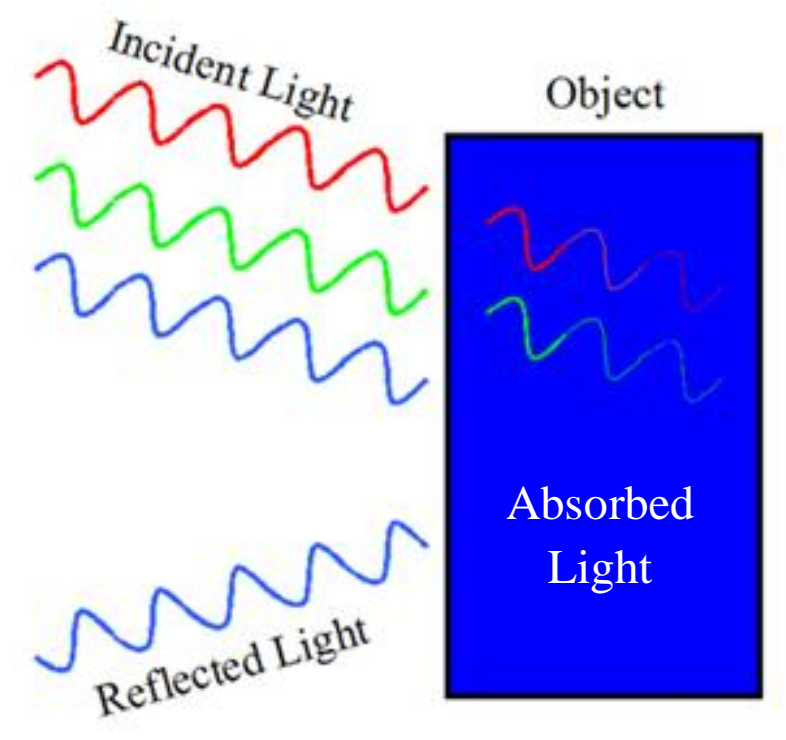

Figure 7 - Three wavelengths of light (red, green, and blue) are incident upon a blue object. Red and green wavelengths are absorbed by the objects crystal lattice leaving blue light to reflect back toward an observer ${ }_{(14)}$.

An insulator (non-conductor) or intrinsic (undoped) semiconductor exposed to sunlight will absorb some colors of light down to the energy where an incoming photon is unable to excite an electron to a higher energy state. Thus the photon will pass through the object as it cannot interact with orbiting electrons ${ }_{(15)}$. In this instance the wavelengths which do not pass through are either absorbed, reflected, or scattered. The wavelengths which make it back to the observer determine the color seen. If an illuminating source is missing much of the wavelengths that would normally be reflected back to the observer the objects color will change since color is a function of wavelength and intensity (see introduction to Chapter IV). There are other reasons why an object may appear a given color, such as preference to reflect or absorb certain wavelengths more so than others or the degree to which wavelengths are scattered. The color rendering index (CRI) is a 
quantifiable value based on a measurement process that has been standardized to determine how much a light source deviates from the sun. The test involves measuring the chromaticity values reflected off of eight samples (selected to cover a large range of hues) under sunlight or a calibrated incandescent source. The illumination source tested must be at the same correlated color temperature (CCT) as the sunlight being replicated. Then the chromaticity values of the eight samples are again measured and the distance they each deviate from their respective value in sunlight is calculated. These eight deviations are averaged and multiplied by 4.6 (scaling factor) and subtracted from 100 to yield an individual number for each sample. The average of that number yields the CRI value with 100 representing sunlight and 0 representing the poorest ability to illuminate the true colors of an object $(16)$.

This information is important when developing an illumination source. In general there are two forms of white LEDs. One method is to use a combination of red, green, and blue LEDs in close proximity to emit what appears to be white light. In addition to the spectrum output issue discussed above, a light source of this nature uses three different LEDs which will degrade at different rates. Overtime this will cause a shift in color values favoring the LED color which degrades at a rate slower than the others. The alternative method, discussed previously, is the pcLED. One advantage of this method is that each series of dies may be placed in whatever configuration is necessary without affecting the overall color value. Whereas red-green-blue (RGB) LED combinations require specific positioning so that white light is apparent. Another benefit of a pcLED is that the phosphor emits light over a broad range of wavelengths which increases the 
sources ability to illuminate colored objects correctly. For instance, a RGB LED may have a CRI near 20 whereas a pcLED may be closer to 90 . This configuration is also more economical than the multi-LED devices $(17)$. 


\section{CHAPTER III: PRINCIPLES OF LUMINESCENCE}

\section{Crystallography}

With any investigation into the properties of phosphors an understanding of the crystallographic structure they form is necessary. Solids may be divided into crystalline, amorphous, or a combination of both when depicting their arrangements of atoms. These categories may be determined by the amount of long and short range order within a structure (Figure 8).
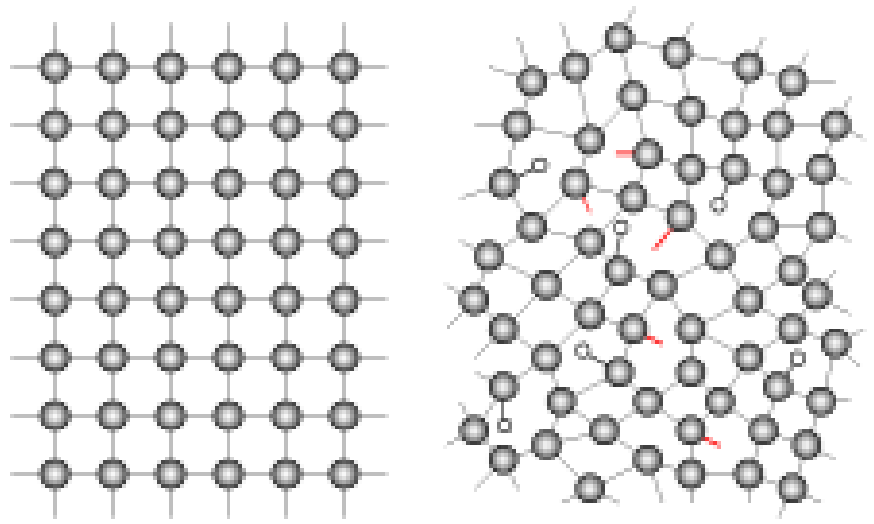

Figure 8 - The left-hand side shows a crystalline region of a material (short range order) whereas the right-hand side represents an amorphous region. If much of the remainder of the material maintains the crystalline structure then long range order is observed ${ }_{(18)}$.

For the purpose of this document, all phosphors will be considered to have a crystalline structure as opposed to phosphors with glass (amorphous) host lattices. Bonding between these atoms may consist of ionic, metallic, covalent, and Van der Waals depending upon the present atoms. Materials discussed within this section will be represented by being comprised of ionic bonds where the dopant resides in an anion position. Ionic bonds hold a crystal lattice together by enabling a cation (atom with one less electron) to share the extra atom from an anion ${ }_{(19)}$. 
Atoms maintain a distance between their neighbors based upon what enables the lowest energy within that system. Thermal energy within the system enables atoms to vibrate in three dimensions with an average separation of $\mathrm{R}_{0}$, defined as the spacing between the center of one atom and its neighbor's center. Their attraction is coulombic and therefore based upon the arrangements of electrons which orient themselves to fill the lowest allowed energy states in a given system. The spacing between two atoms is maintained mainly by the repulsive force created by the Pauli Exclusion Principle and the attractive force induced from coulombic forces (in the ionically bonded instances). As two atoms move closer to each other the wavefunctions of their inner shell electrons begin to overlap. As the Pauli Exclusion Principle states, no two electrons may have the same quantum numbers. To prevent having identical quantum numbers, electrons begin to fill higher energy levels. The extra energy required to excite an electron into a higher energy level is shown by the repulsive force of the two atoms. Attraction from the coulombic forces is caused by the separation of two oppositely charged atoms $(20)$.

As discussed in the following section, the separation $\mathrm{R}_{0}$, may adjust based upon phonon interaction or electron stimulation. Phonons provide a given system with additional energy by means of lattice vibrations which change the average separation to some value $\mathrm{R}$, that deviates from the equilibrium position. During electron stimulation, a different level occupation causes a change in the columbic forces resulting in a new equilibrium separation (Figure 9). 


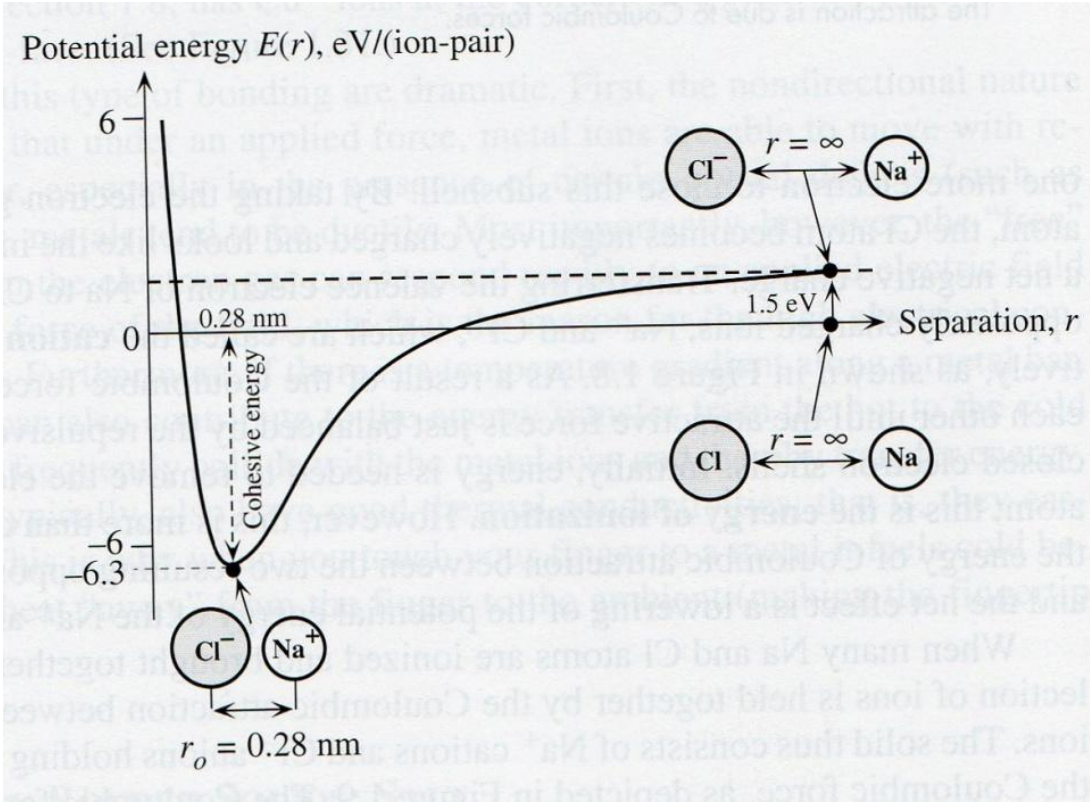

Figure 9 - Sketch of the potential energy per solid pair of $\mathrm{NaCl}$ (salt). Equilibrium exists at the lowest potential energy level $\left(r_{0}=0.28 \mathrm{~nm}\right)$, two ions infinitely far away have a potential energy of $1.52 \mathrm{eV}$ per pair, and two neutral atoms infinitely far away have no potential energy. Additional energy in the equilibrium system will cause a shift in the equilibrium distance. Note: $r$ in image is the same as $\mathbf{R}$ in this text ${ }_{(21)}$.

What enables phosphors to luminesce is the addition of dopants, or impurity atoms, within the host lattice. Impurity atoms typically account for somewhere on the order of a hundredth of a percent of the total atomic population of a given phosphor. They reside at some position of the crystal lattice via substitution. A strain is placed upon the lattice at that point due to the abnormally sized impurity atom. It is the distortion in the crystal lattice and additional electron energy states which enable photon emission.

\section{Basics of Solid State Physics}

In order to understand the electron transitions occurring in a luminescent material, one must consider band theory in solids. To characterize the relative energy level of an outer shell electron around an atom we may consider the isolated atom point of view. In a 
system without external interaction, an electron may occupy a state $n \geq 1$ (known as the principle quantum number). When examining the periodic table this number correlates to the row number an atom resides in (Figure 10). For a given principle quantum number, the electron also occupies an angular momentum quantum number defined as $0 \leq \ell \leq n-$ 1. Where $\ell=0$ is defined as the $s$-state, $\ell=1$ the $p$-state, $\ell=2$ the $d$-state, $\ell=3$ the $f$-state, and $\ell=4$ the $g$-state as depicted on the periodic table of elements. Further dividing these potential states are the magnetic quantum numbers determined by the equation $-\ell \leq m \leq \ell$. The last state considered in this analysis is the spin state of the electron. For any given combination of states determined by the $n, \boldsymbol{\ell}$, and $m$ quantum numbers, a spin up or spin down state may also exist. This is represented by $s= \pm 1 / 2$. The energy released in the form of a photon is dependent on what are the filled and unfilled electron states of the dopant atoms. For instance, an excited electron from a cerium atom (the luminescent center for YAG:Ce phosphor) may occupy a 5d state. This implies that it has a principle quantum number of ' 5 ' and a momentum quantum number of '2.' Depending upon the excitation energy, the electron may occupy the first filled $5 \mathrm{~d}$ state which has a magnetic quantum number of ' -2 ' and a spin of ' $+1 / 2$.' After approximately 70 nanoseconds (decay time for YAG:Ce) the electron falls to the $4 \mathrm{f}$ state where it now has a principle quantum number of ' 4 ' and a momentum quantum number of '3.' Since the electron now occupies its equilibrium position the magnetic quantum numbers and spin states can be determined by the periodic table where they are ' -3 ' and ' $+1 / 2$ ' respectively $(15)$. 


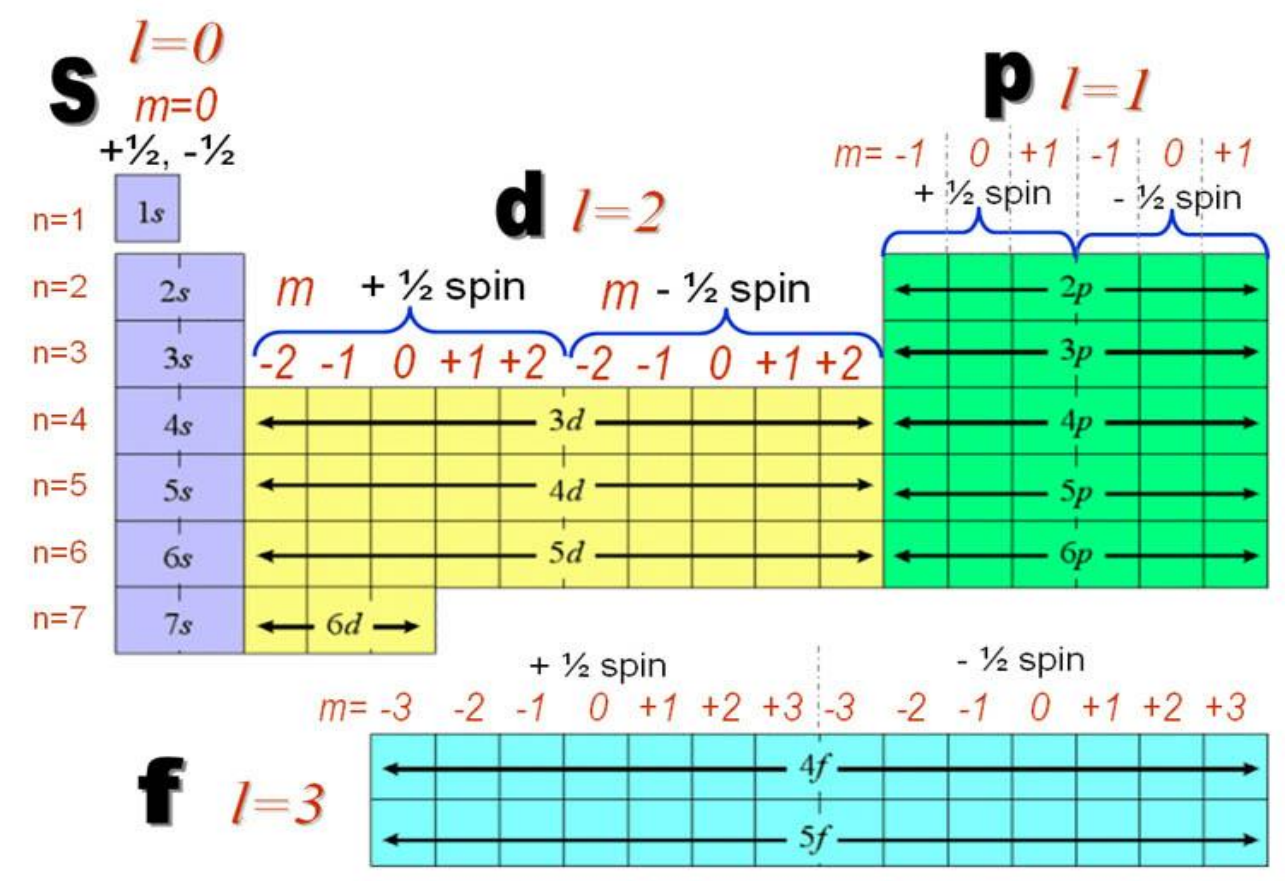

Figure 10 - A representation of the various quantum numbers observed in ground state atoms. Alkali and alkaline earth metals have outer shell electrons in a s-state. Transition metals are in the process of filling their $\boldsymbol{d}$-orbitals. Post-transition metals, metalloids, and nonmetals are depicted by filling their $p$-orbitals. The materials in particular interest for many phosphors are lanthanoids and actinoids which contain electrons in $f$-orbitals $(22)$.

The Pauli Exclusion Principle links individual atomic energies into a bulk form developed in a solid material. It states that no two electrons may have all the same quantum numbers in a given system. A band of energies comes about from the large quantities of electrons in a bulk material trying to occupy similar states at the same time.

A reduced form of electrons energy versus momentum diagram (configurational coordinate diagram) displays the allowed states in a lattice (Figure 11). At the uppermost energy states exists a conduction band and a valence band which may or may not overlap depending upon the material. When dopants are added to a material they provide additional states for an electron to exist in. In the case of visible light emitting phosphors, they create a gap between two electron states that is on the order of the energy 
of visible light. In many phosphors, each dopant position in the host lattice is referred to as the luminescent center due to the fact that it is the electrons at that position which emit visible photons during the luminescence process ${ }_{(15)}$.

\section{Nomenclature}

There are a few important terms utilized in the following sections to describe luminescence in a phosphor. When mentioning energy transitions, the terms electronic, vibrational, and vibronic are used (Figure 11).

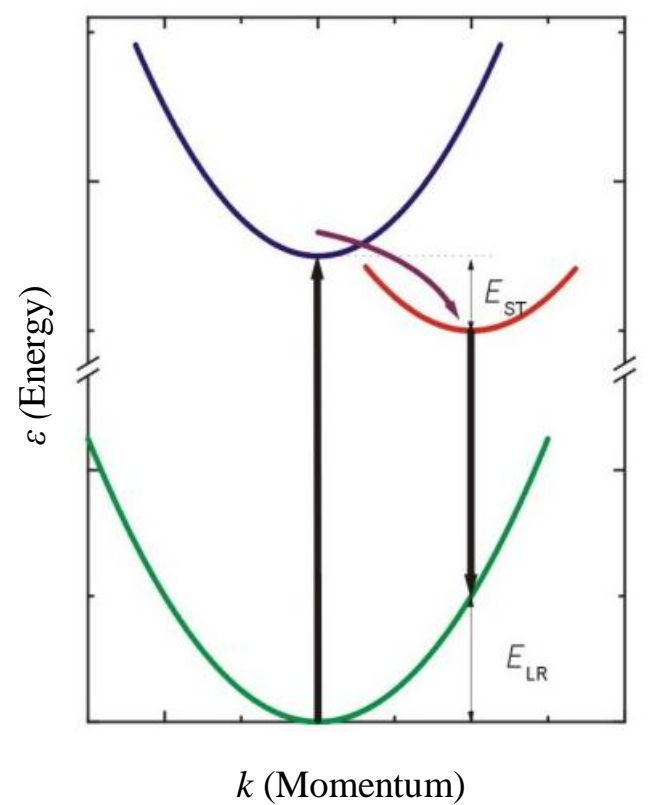

Figure 11 - An $\varepsilon$ versus $k$ diagram displaying the quantum energy states which an electron may fill. Vertical lines represent electronic transitions where all changes in energy occur with adjustments in electron states. Vibrational energy transitions are depicted by changes in momentum (horizontal motion) and involve energy dissipation by means of lattice vibrations. The combination of both is referred to as a vibronic transition ${ }_{(23)}$.

Electronic transitions refer to the decay of an electron from an excited state to a ground state which enabled a photon emission and no phonon release. The somewhat opposite approach to reaching a ground state is the vibrational transition which occurs when an electron does not have to drop an energy gap to move from its excited state back to its 
ground state and therefore does not release a photon. Instead the electron decays in such a manner that relaxes its excess energy in the form of phonons. The combination of these two methods is labeled vibronic transition and it can be seen often with the luminescence behavior of rare earth elements.

Crystal field theory is also of great importance when understanding luminescence. It may be used to describe the strength of a bond between a ligand (ion bound to a central atom) and another atom. The theory states that as an ion approaches the central atom, electrons in various orbitals of the central atom will feel different levels of repulsion from the electrons in the ion. The changes in repulsive forces felt at the various orbital levels causes a splitting in energy resulting in different electron energy states as opposed to the free ion model. This is an essential model to consider as it is able to explain why different spectrums arise from elements in one host lattice compared to the same elements in another host lattice.

${ }^{` 2} \mathrm{~F}_{5 / 2}$ ' is a typical configuration for representing an occupied or potential energy level. The first number (2) represents the principle quantum number. The symbol, in this instance $(\mathrm{F})$, describes the total angular momentum which is a combination of the orbital angular momentum and the spin state. As with the orbital angular momentum values, $\mathrm{L}=$ 0 is defined as $(\mathrm{S}), \mathrm{L}=1(\mathrm{P}), \mathrm{L}=2(\mathrm{D}), \mathrm{L}=3(\mathrm{~F})$, and $\mathrm{L}=4(\mathrm{G})$. The subscript indicates the energy level where $|\boldsymbol{\ell}-\mathrm{s}| \leq \boldsymbol{j} \leq \boldsymbol{\ell}+\mathrm{s}$.

Spin multiplicity also plays a role in radiative emission in phosphors. It is represented with a superscript before the total angular momentum symbol such as $\left({ }^{8} \mathrm{~S}\right)$ which 
indicates a spin octet. Spin multiplicity describes the number of possible arrangements (up-up, up-down, down-down, etc.) that a given amount of electrons may be in (24).

\section{Selection Rules}

The intensity of light emitted from a phosphor is highly dependent upon whether the transition which occurred was allowed or forbidden. Transitions which are allowed will occur more frequently than forbidden transitions. This is essentially due to the fact that if a forbidden transition occurs it is a result from lattice vibrations or other phenomenon which provided slightly more energy to overcome the unlikely transitions based upon various rules. As a result, the time it takes to enable a forbidden transition to occur may be much greater than allowed transitions. Objects which glow are comprised with phosphors which exhibit this phenomena of an extended decay time.

Spin selection rule and parity selection rule make up the two most important rules when understanding why an emitted wavelength occurs long after excitation took place or why the intensity is small relative to the rest of the emitted spectrum. The spin selection rule forbids electrons from transitioning between two energy levels which have separate spin states (i.e. $+1 / 2$ transition to $-1 / 2$ ). The parity selection rule forbids the electronic transition within the $d$ shell, $f$ shell, and between the $d$ and $s$ shell $(24)$.

\section{Coupling Between Electrons and Vibrations of the Center}

Width of the luminescence spectrum depends upon the strength of the coupling between electrons and vibrations about the luminescent center. Again, the configurational coordinate diagram is helpful when visualizing this phenomena ${ }_{(23)}$. Recall that the value

$\mathrm{R}$ is the structural parameter which exists during vibration while $\mathrm{R}_{0}$ is the structural 
parameter at rest. $\mathrm{R}$ values which deviate from $\mathrm{R}_{0}$ result in less probable transitions for greater deviations (Larger $\Delta \mathrm{R}$ ). This is reflected in a spectrum with wider absorption or emission bands. When $\Delta \mathrm{R}$ is equal to zero fully electronic transitions occur resulting in a non-phonon production and therefore a weak-coupling relationship between electrons and the vibrations about the luminescent centers. On the opposite end if $\Delta \mathrm{R}$ is much larger than 0 a large amount of phonon production is essential for an electron transition to occur yielding a strong-coupling scheme. In between these extremes, when $\Delta \mathrm{R}$ is larger than 0 , is referred to as an intermediate coupling scheme ${ }_{(24)}$.

\section{Absorption of Excitation Energy}

Two major characteristics are apparent when comparing various absorption spectrums. Specifically the width and intensity of specific portions of the spectrum are able to tell a lot about the material in question (Figure 12). 


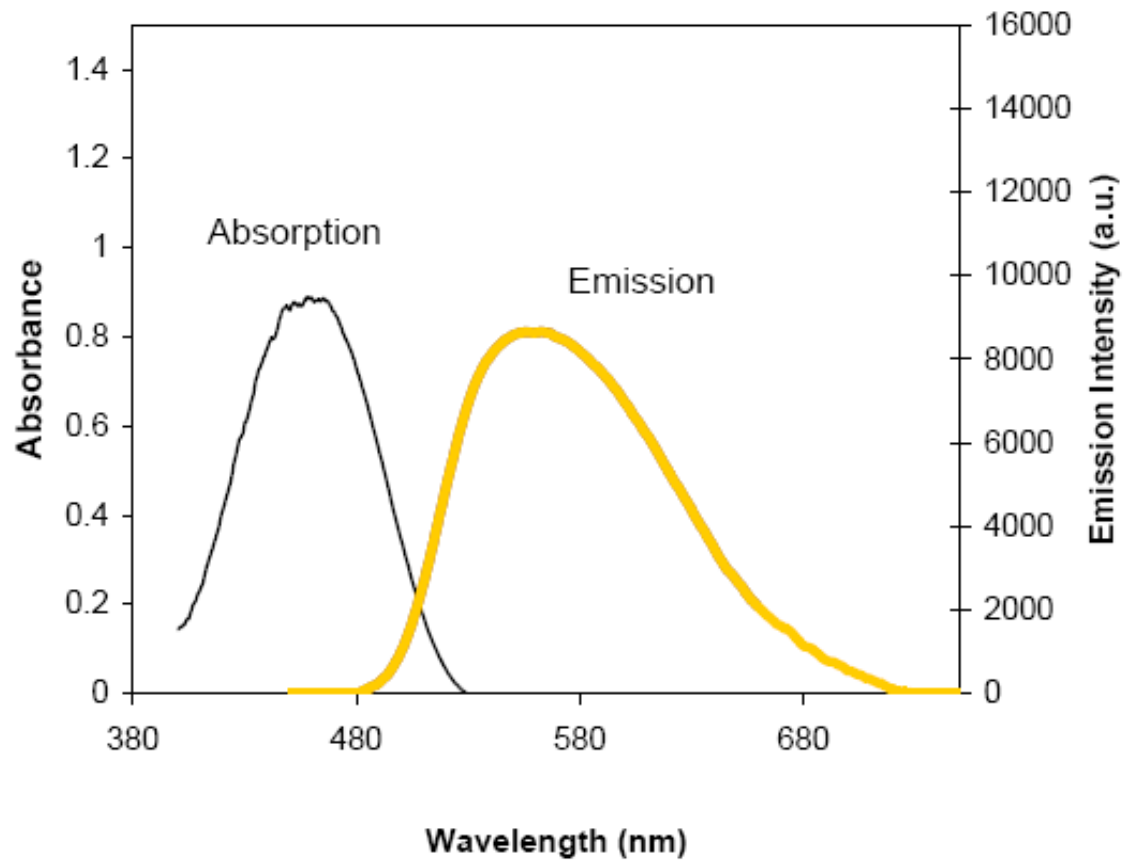

Figure 12 - The absorption and emission spectrum of YAG:Ce provided by PhosphorTech. The various widths and heights of theses spectrums indicate the interaction between the host lattice and $\operatorname{dopant}_{(25) \cdot}$.

Broad and Narrow Spectrums

As we have discussed previously, how narrow or broad an absorption band appears stems from $\Delta \mathrm{R}$. The broader the spectrum, the larger $\Delta \mathrm{R}$ is, and therefore the greater the offset between lowest vibrational levels in the ground and excited states. This offset arises from a shift in the rest position of the center. Chemical bonds within the crystal lattice often change (generally become weaker) when an orbiting electron transitions from a ground state to an excited state. The change in bond strength results in a shift of equilibrium distance between atomic positions $(\Delta \mathrm{R}>0)$, as compared to the initial vibrational state $(\Delta \mathrm{R}>0)$ which broadens the absorption band $(24)$. 
Magnitude of Intensity

There are a few common physical phenomena which enable forbidden transitions to occur in solids. One is spin orbit coupling where the electromagnetic field produced between the nucleus and electrons spin may result in slightly smaller or larger electron atomic energy levels depending upon its spin direction. Electron-vibration coupling may also influence the energy state of an electron due to phonon movement. Uneven crystal field terms, which occur when a dopant ion occupies a low symmetry point in the lattice, may enable low intensity emission due to a relaxation in the parity rule. In some instances the dopant concentration may be small enough that even allowed transitions occur at a small intensity (24).

\section{Host Lattice Influence}

The host lattice may have a dramatic influence on the ability of a phosphor to absorb photons. If a phosphor absorbs lower wavelengths of light it implies that more energy is required to remove an electron from the host atom to the dopant atom. This is referred to as the charge-transfer band shift. Another form of host lattice influence is in the cases where filled $s$ and $p$ orbitals are able to shield weak $f$ transitions in the dopant atom. The result is a narrow and weak emission profile. Covalency also plays an important role when understanding the influence a host lattice may have on its dopant. If electrons are spread out over wider orbitals they will have less affect on the host lattice. Higher causes a shift to lower energies of the charge-transfer transitions due to a reduction in electronegativity between constituting ions. This results in absorptions occurring more frequently at higher wavelengths. The strength and symmetry of the crystal field may also influence the spectrum and usually change depending upon the relative size of the 
dopant ion relative to the host lattice ions. For instance, if a larger ion occupies a position where a smaller atom would normally be, a stronger crystal field forms and may cause splitting of certain optical transitions or may even lift the parity selection rule. Stronger crystal fields also enable optical transitions to occur at higher energies. Phosphor powders add another important physical trait to consider. External, as well as internal, surfaces may be relatively large enough to provide inhomogeneous broadening because of different energies available to dopants which reside in point defects or on the surface. The crystal field and covalency in those points differ from the bulk material resulting in additional electron energy states available. This is apparent in glasses where optical centers differ from site to site due to the amorphous configuration of atoms creating variations in $\Delta \mathrm{R}_{(24)}$.

\section{Emission: Radiative}

Emission from an excited electron may be radiative or non-radiative (i.e. emit a photon or dissipate energy with a phonon). Radiative electron decay is the phenomenon of interest for the purpose of this paper.

\section{Brief Review of Absorption}

In this explanation of the absorption-emission cycle we will assume that there is an offset between parabolas of the ground and excited state. Upon electron excitation, there is no expansion of the luminescent center. Eventually the luminescent center will return to the lowest vibrational level of the excited state by giving up excess energy to the surroundings. In other words the interatomic distances equal the equilibrium distances of 
the excited state. This is the value $\Delta \mathrm{R}$ which was covered in the previous sections. This entire process is referred to as relaxation and usually incorporates no emission $(24)$.

\section{Specific Tendencies}

Emission then occurs spontaneously and we will assume in the absence of a radiation field for the following discussion. This is the instance when the excited electron decays to the lowest vibrational level of the ground state. $\Delta \mathrm{R}$ will then contract to the equilibrium distance of the ground state and the process may be repeated again. The spectrum of the emission is often compared to the spectrum of the absorption where the peak-to-peak difference is known as Stokes shift. It is the energy difference between the maximum of the lowest excitation band and the emission band. By looking at the magnitude of stokes shift and the width of the optical band we can make assumptions about the changes in the structural parameter. Essentially larger $\Delta \mathrm{R}$ equates to larger stokes shift and broader optical bands. We may also determine the strength of the electron-lattice couple, also known as the Huang-Rhys Coupling Constant, as it is proportional to $\Delta \mathrm{R}$.

Emission profiles can also tell us about the electron-lattice coupling strength. For instance $\Delta \mathrm{R}$, and therefore stokes shift, becomes smaller with stiffer surroundings of the luminescent center. The intensity of the optical emission is related to the lifetime for which it takes an electron to decay. More intense emissions will have shorter decay times than less intense emissions. In the instance of an allowed emission transition the decay time will be short (on the order of $10^{-8}$ seconds) where in contrast forbidden transitions will display much longer decay times (on the order of $10^{-3}$ seconds) (24). 


\section{CHAPTER IV: TESTING AND CHARACTERIZATION EQUIPMENT}

\section{Introduction}

Characterizing an illumination source required the ability to measure the wavelength and intensity of emitted light at specific points above the source. Light emitted from an LED is much more directional then an incandescent bulb. An incandescent bulb emits photons from all directions around its tungsten filament. That characteristic enables light intensities and colors to be more uniform at any angle around the bulb. A LED on the other hand contains objects such as heat sinks, interfaces for conducting layers, and other materials which yield preferential directions of light output due to a more optimal light path created by the various indices of refractions all in one package. Cree and Philips Lumileds brand LEDs tested in this paper display the most luminance directly above the die whereas some deviant angle from that point will have a relatively decreased luminance value. This positional change in intensity also causes color variations across a uniform phosphor layer which will be discussed in following chapters. The need to characterize the LED at different positions other than directly above the die prompted the development for a two dimensional linear stage set up or use of an integrating sphere depending upon which tests were to be conducted.

Characterizing LED configurations with various phosphors required that colors be reported. The $1931 \mathrm{CIE} 2^{\circ}$ Standard Observer was utilized to provide quantitative color measurements for each test enabling observations to be independent from human color interpretation. It is characterized by three color matching functions known as tristimulus 
values. Excel Macros were used to convert measured intensity and wavelength values outputted from a spectrophotometer into $\mathrm{x}$ and $\mathrm{y}$ chromaticity values using equations shown below.

$$
\begin{aligned}
& X=\int_{0}^{\infty} I(\lambda) \bar{x}(\lambda) d \lambda \\
& Y=\int_{0}^{\infty} I(\lambda) \bar{y}(\lambda) d \lambda \\
& Z=\int_{0}^{\infty} I(\lambda) \bar{z}(\lambda) d \lambda \\
& x=\frac{X}{X+Y+Z} \\
& y=\frac{X}{X+Y+Z}
\end{aligned}
$$

Equation 6

Where $\mathrm{X}, \mathrm{Y}$, and $\mathrm{Z}$ are tristimulus values based upon light intensity (I) and colormatching functions $(\bar{x}, \bar{y}$, and $\bar{z})$. The code established in Excel approximated the tristimulus values with wavelengths of every $10 \mathrm{~nm}$. $\mathrm{x}$ and $\mathrm{y}$ are chromaticity coordinate values, which are defined by a region, made up from the color values of individual wavelengths of light (Figure 13). 


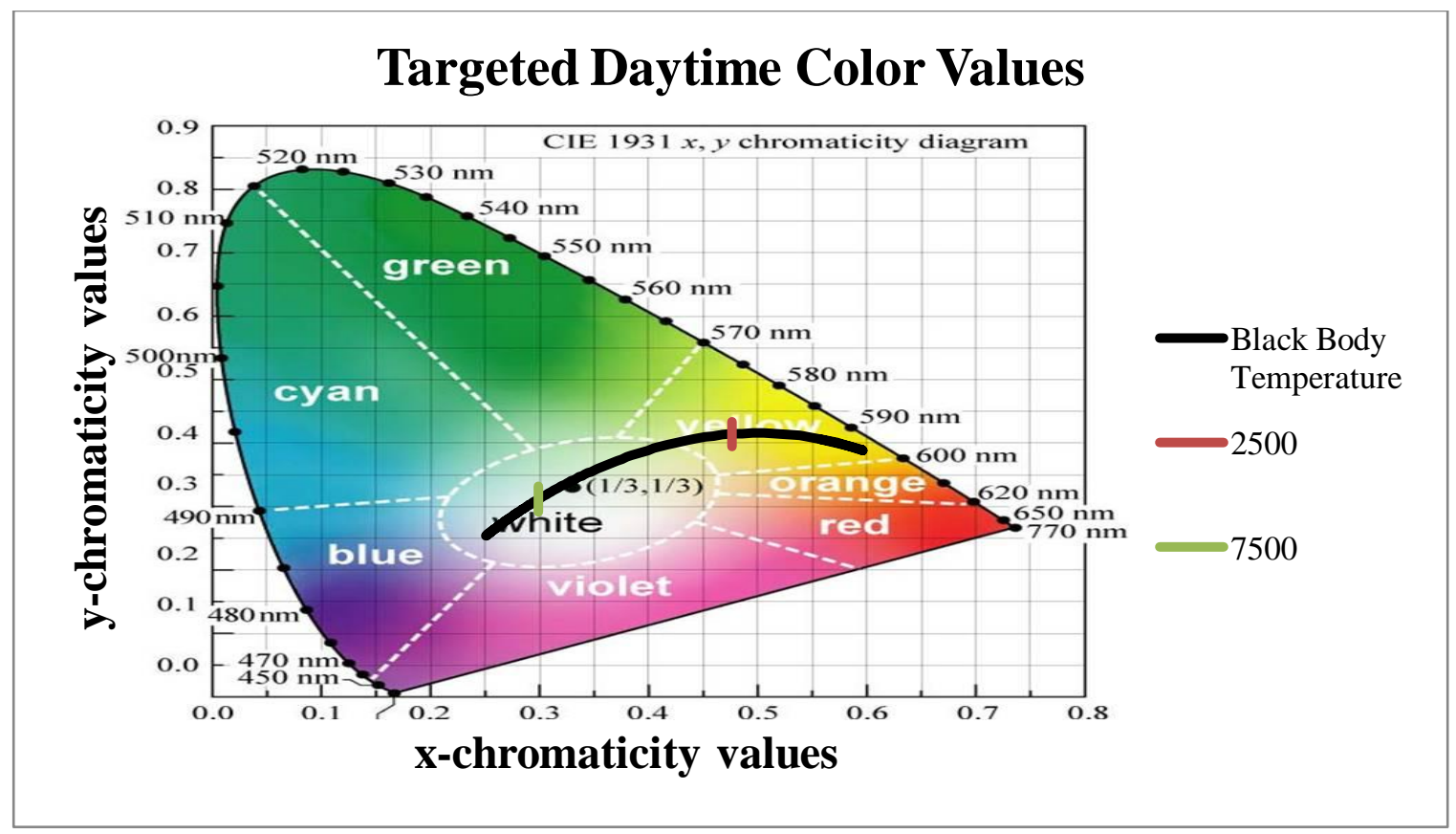

Figure 13 - This thesis investigates the feasibility of mimicking the Kelvin scale between 2500 and 7500. The two temperatures on the chromaticity diagram represent the color that would be visible if an object (such as a tungsten filament in an incandescent bulb or steel in a mill) were heated up to those temperatures. The black body temperature curve extends from 1000 Kelvin (Orange) to 25000 Kelvin (Blue).

\section{Testing Apparatus}

The left-hand side consisted of a combination of Thorlabs and Edmund Optics optical products (Figure 14). An integrating sphere, beam splitter, and two Thorlabs LEDs are mounted on the optical stage. A combination of Newport linear stages, plates, and right angle brackets enabled optical alignment of the optics tube assembly. A micrometer on the vertical assembly allowed adjustment necessary for best placement of the focal point. $\mathrm{x}$ and $\mathrm{y}$ movement during mapping is possible due to two linear stages mounted perpendicular to one another and positioned below the optics tube. On the right-hand side of the testing apparatus sat two data acquisition units (DAQs), a linear motion controller, two spectrometers, four power supplies, and a computer monitor (Figure 15). 


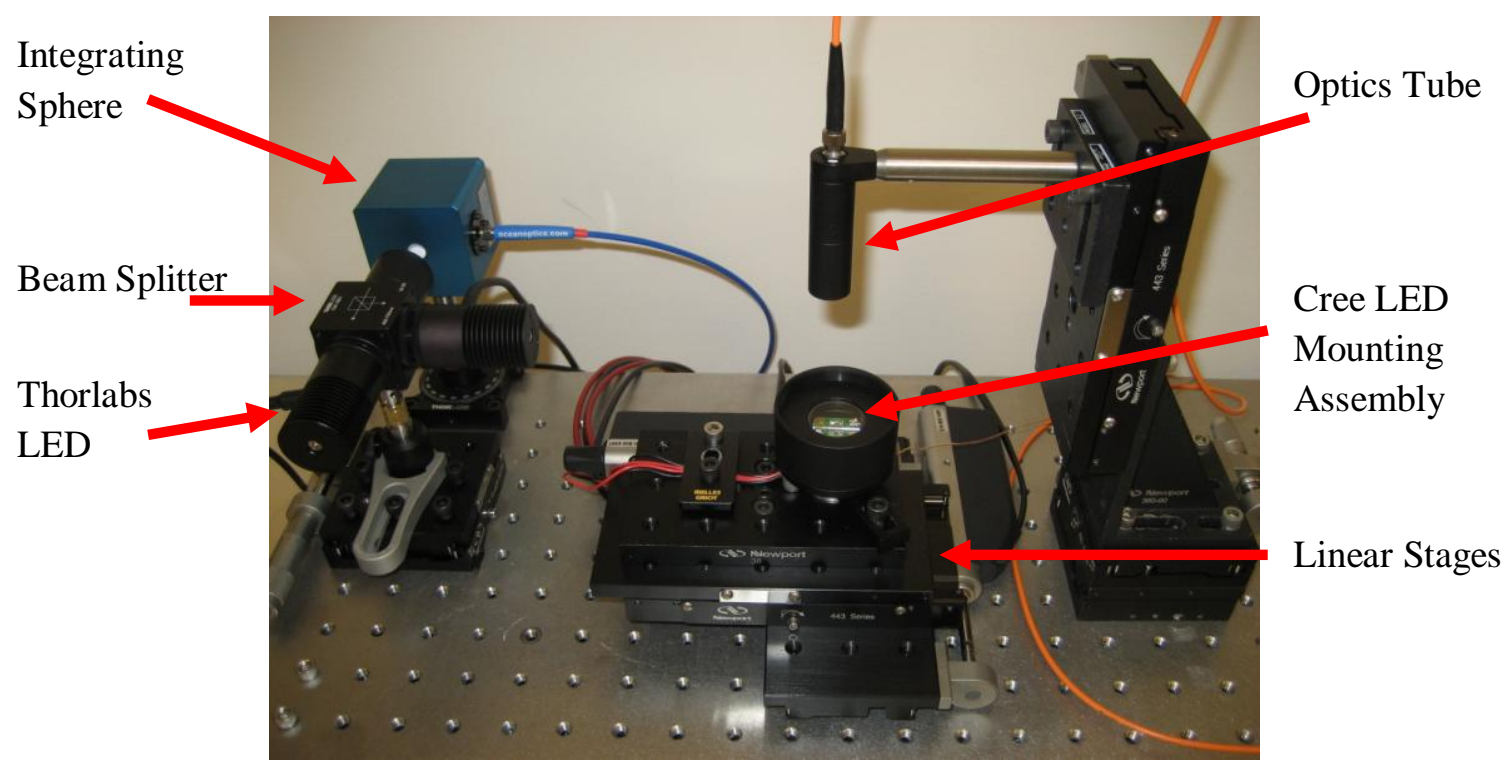

Figure 14 - Each set up is mounted on top of a Thorlabs optics table. On the left hand side of the image sits a beam splitter positioned to combine light from both Thorlabs LEDs and direct it into the center of the integrating sphere. The center of the images displays Cree LEDs mounted on linear stages where they are capable of moving under an optics tube.

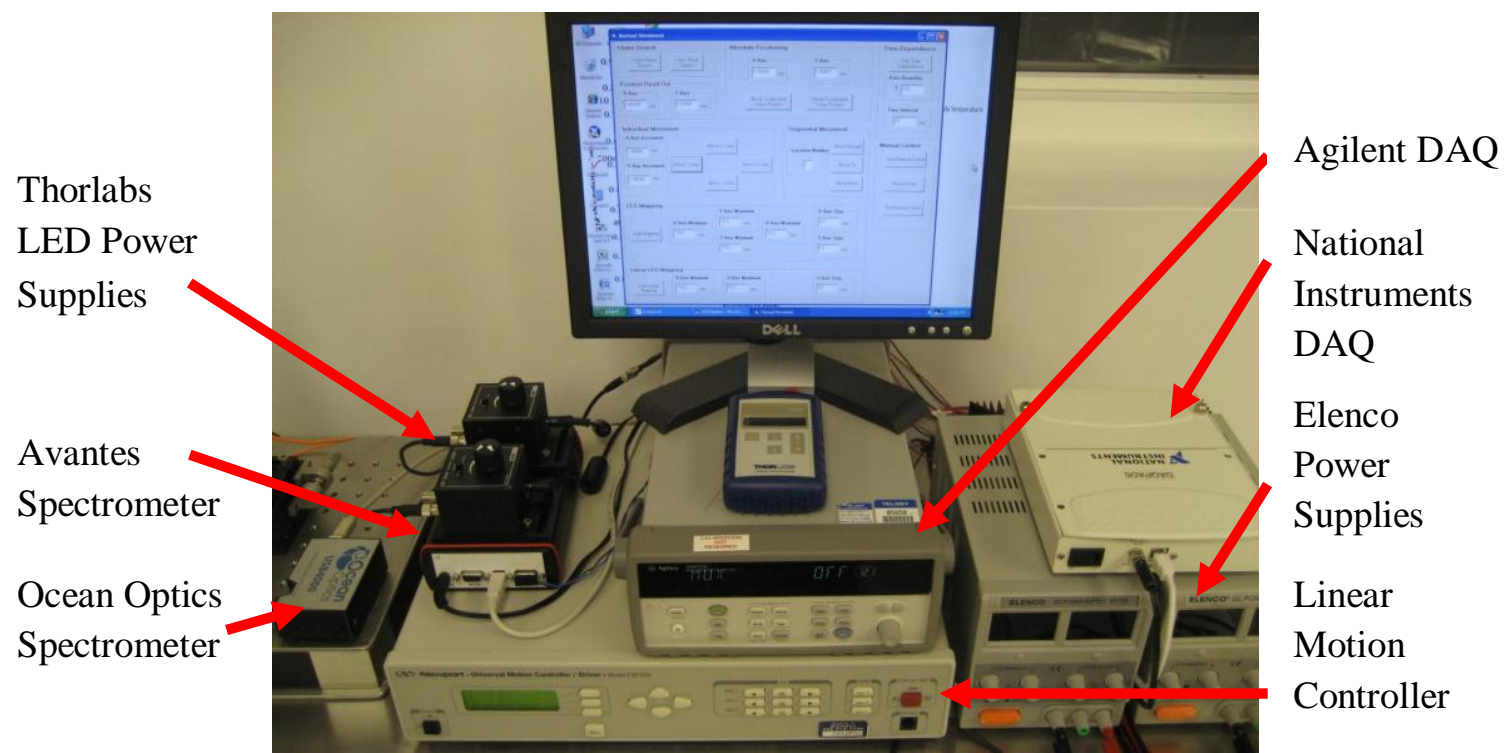

Figure 15 - In the center of the images sits a computer monitor and Thorlabs power meter atop an Agilent DAQ. To their left sits two Thorlabs power supplies atop an Avantes spectrometer. All that equipment is positioned on a Newport linear motion controller which is surrounded by an Ocean Optics spectrometer and two Elenco power supplies. A National Instruments DAQ rests on top of the Elenco Power supplies. 


\section{Linear Motion Controllers}

Two dimensional mapping was made possible with two Newport linear motion motors attached to two Newport linear motion stages. Motors were controlled using a Newport Universal Motion Controller/Driver Model ESP300. Programming in Microsoft Visual Basic 6.0 provided the means to interface the controller to the computer for preprogrammed experiments.

\section{Spectrometer}

To measure the electromagnetic spectrum from the LED and phosphor particles an Avantes AvaSpec-2048 spectrometer was used. A $200 \mu \mathrm{m}$ fiber optic cable with SMA connectors routed the light to the device. The specific model utilizes AvaSoft 6.2 Full 2004 software which has the ability to display up to 16383 counts per pixel and adjust the integration time from 2 milliseconds to 60 seconds. The software also enables external triggering which was essential for testing being done on this project. The spectrometer has the capability of 0.04 to $20 \mathrm{~nm}$ full width at half maximum (FWHM) resolution while detecting wavelengths from 200 to $1100 \mathrm{~nm}$ (Ultra Violet to Near Infrared range). The setup used a $200 \mu \mathrm{m}$ fiber optic cable with a grating of 300 lines per millimeter resulting in an $8.0 \mathrm{~nm}$ FWHM resolution.

\section{Data Acquisition Unit (DAQ)}

To trigger the Avantes spectrometer at the precise moments necessary for obtaining spectrums at specific instances, a National Instruments DAQPad-6016 was being used. The unit has the capability of sending a $5 \mathrm{~V}$ signal to the spectrometers RS-232 port. DAQPad-6016 is compatible with Microsoft Visual Basic which was utilized to signal the DAQ at the required times. 
A second DAQ (Agilent Model \#34970A) recorded two thermocouple readings as well as voltage and amperage readings from both Thorlabs and Cree LEDs. Connections were made using one male and one female Thorlabs LEDD1 style four pin connector and soldering wires to form a T-fitting between the DAQ, Power Supply, and LED unit. Two of these assemblies were produced for up to two simultaneous LED input power measurements during a given experiment. A similar set up was manufactured for the Cree LEDs but a series of banana plugs were used instead of the specific four pin connector needed for Thorlabs LED products.

Cree and Thorlabs LEDs

Thorlabs Power Supply and Mounting Techniques

Thorlabs LED Driver model LEDD1 powers the Thorlabs blue and UV LEDs. Current can be adjusted from 0 to $0.7 \mathrm{~A}$ and is set via a knob atop the device. This driver is the recommended power supply for Thorlabs high brightness LEDs which are capable of withstanding 0.7 A. Thorlabs LEDs were supplied mounted in an assembly with multiple heat dissipating fins. These fins were pressed against two allen-head bolts on a linear stage and held down with a pivoting bar on the opposite side. This simplistic method to secure the LED housing was sufficient for all movements of the linear stages during testing.

Cree Power Supply and Mounting Techniques

An Elenco XP752 DC power supply was used to supply the blue LED with $2.99 \mathrm{~V}$ at 3.0

A to $3.51 \mathrm{~V}$ at $30.0 \mathrm{~A}$ and anything in between. A duplicate power supply provided the UV LED with $3.21 \mathrm{~V}$ at $25.0 \mathrm{~A}$ to $3.40 \mathrm{~V}$ at $42.0 \mathrm{~A}$ and anything in between. An 
aluminum substrate was manufactured to fit within a one inch diameter cylindrical threaded optics tube sold by Thorlabs. Two opposite sides were machined flat to provide clearance for power supply wires. A rail was left in the center, wide enough for two LEDs to be mounted on, and also tall enough to be near the thickness of an adhered PCB board. The adhered PCB boards were placed on each side of the rail and provided the electrical path between the power supply wires and the wire bonds to the LEDs themselves (Figures 16, 17). No epoxy was used atop each LED because of an original goal to study long term degradation effects of phosphors not encapsulated in an epoxy. This caused wire bonds to be exposed making the overall assembly fragile. A two inch cylinder was eventually used to negate the fragile nature of the assembly from external movement of the power wires or placement of the phosphor/PDMS substrates.

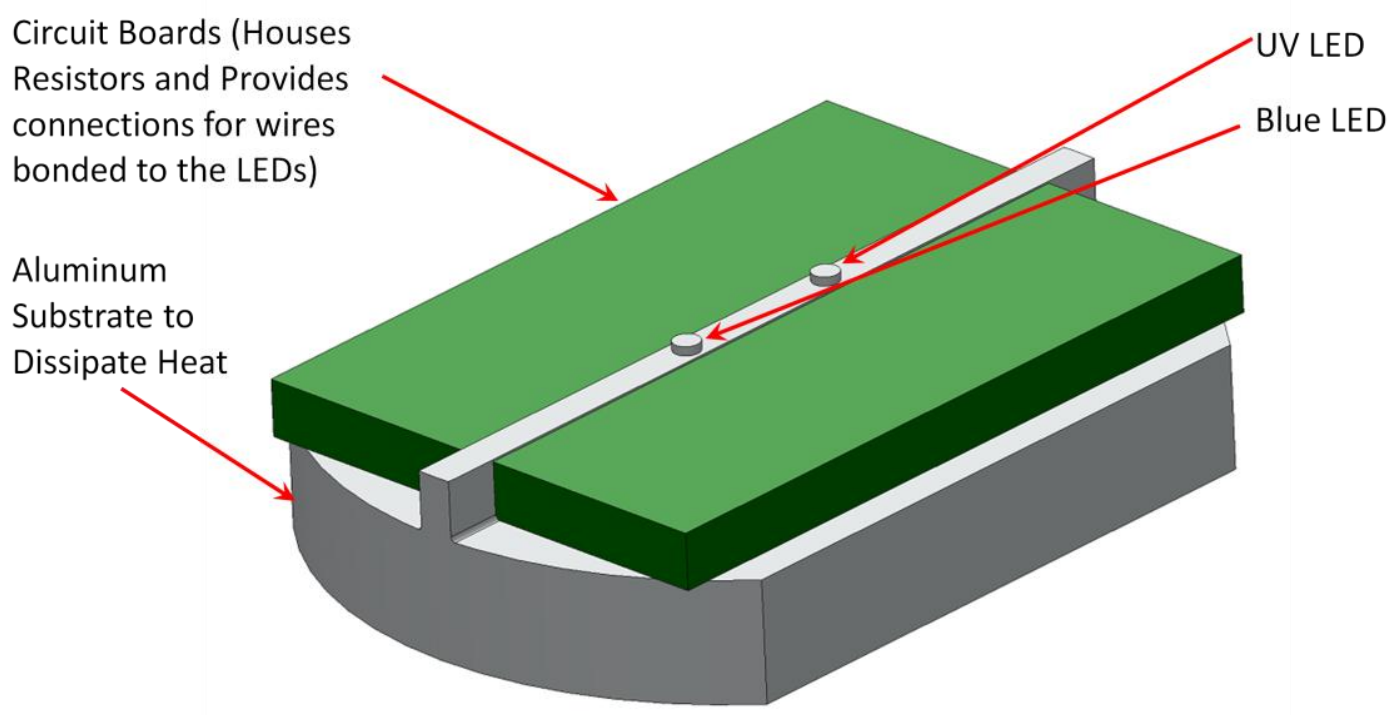

Figure 16 - A one-inch diameter aluminum substrate with a single rail down the diameter to allow for two LEDs to be mounted. Wire bonds extended from the top of each LED die to the corresponding PCB board (green rectangles). The flat portion on the aluminum substrate sides enables power wires to be routed without interfering with one-inch diameter optics tubes. 


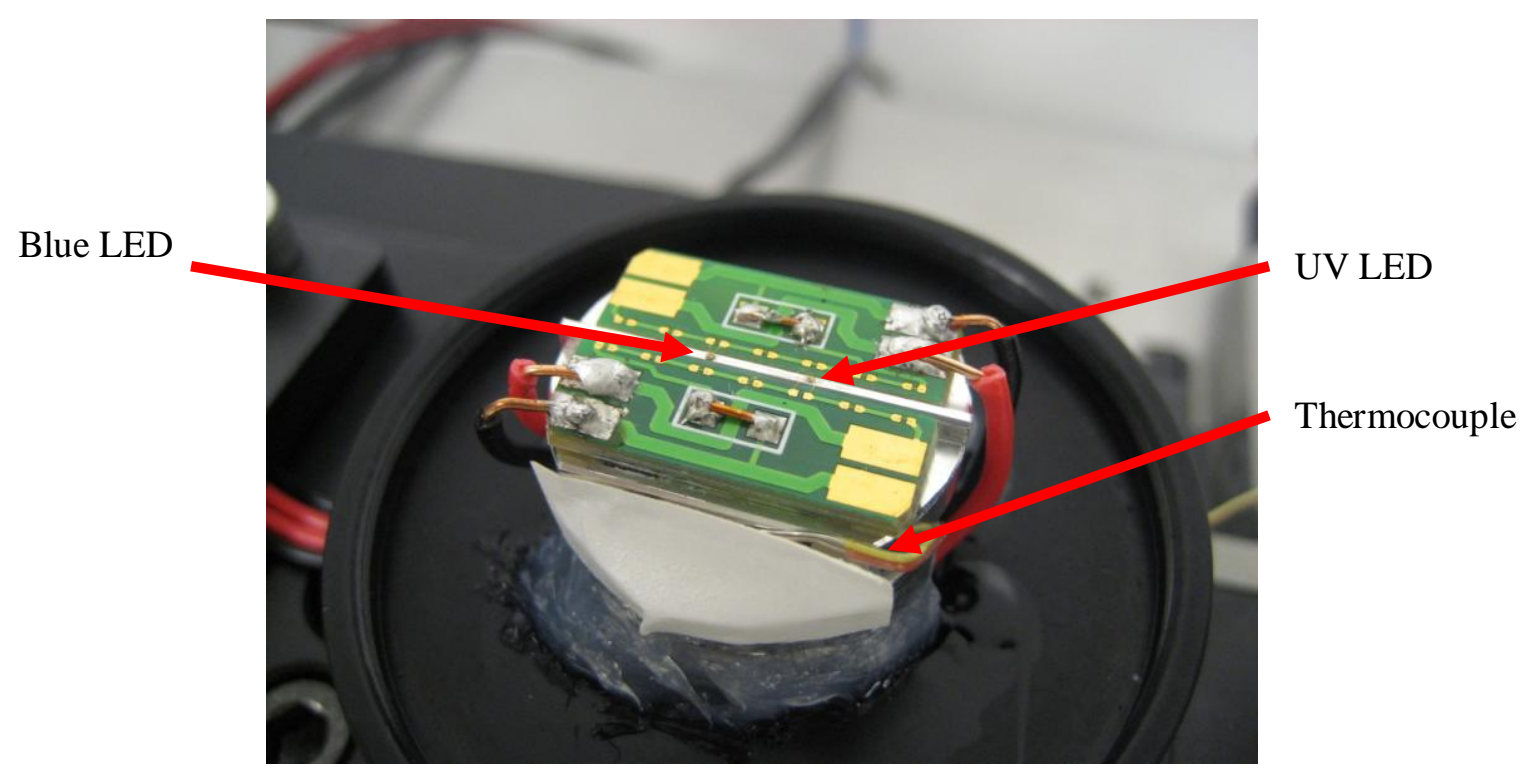

Figure 17 - An image of the actual set up ready for testing. Positive (red) and negative (black) wires come from the corresponding Elenco power supplies. A thermocouple (lower-most wire) monitors the aluminum substrate temperature during testing.

Although the apparatus configuration for Cree LEDs is discussed here, testing is not mentioned in this thesis due to the difficulty for analysis incorporated with this design. The low intensity of each die (relative to Thorlabs LEDs which contain sixteen die), the large offset between each LED, and the fragile nature of the exposed wire bonds made this configuration impractical for initial characterization experiments. Testing eventually indicated that UV light is not beneficial to a multi-Kelvin LED with the design considered for this report, therefore making the Cree-LED set up irrelevant to future studies.

\section{Optics Tube Assembly}

The optics tube assembly consists of two aspheric lenses and two neutral density filters all positioned with Thorlabs lens tubes and their accessories (Figure 18). 


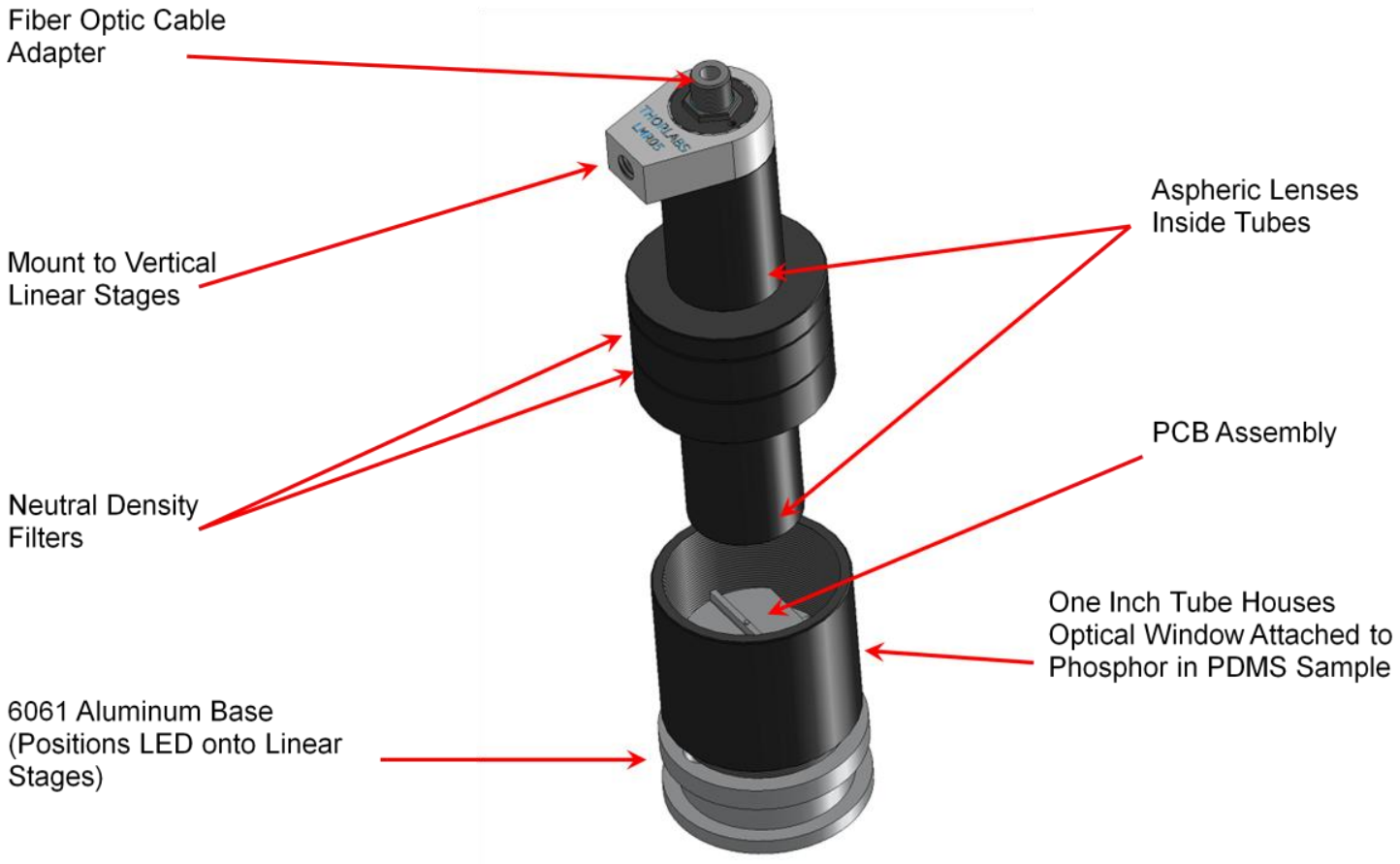

Figure 18 - This Solidworks image displays two separate components. The top piece remains stationary during testing and houses the lenses and neutral density filters. The bottom piece houses the LEDs and sits atop linear stages that move according to the specified program.

The neutral density filters come from Edmund Optics kit \#47-511. Based off the max intensity of the Thorlabs blue LED and the point of saturation for the Avantes Spectrometer, a 1.3 (\#47-208) and a $3.0(\# 47-212)$ filter was selected. The combined selection yields a total of a $43 \mathrm{~dB}$ loss $/ \mathrm{cm}^{2}$ which translates to a $0.005 \%$ Transmission.

Integrating Sphere and Beam Splitter

In order to investigate the behavior of phosphors with intensity adjustments from the blue and UV LEDs, a beam splitter and an integrating sphere were utilized (Figure 19). The beam splitter has the ability to direct $50 \%$ of one light source and $50 \%$ of another light 
source onto one target in what appears to be the same location. This allows for a blue and an UV light source to be mounted perpendicular to each other but produce peak intensities at the same location on a phosphor substrate. This set up minimizes the effects of chromaticity changes caused by spatial displacements with the LEDs. The second important piece of equipment is the integrating sphere. The integrating sphere gathers light from a source and negates the changes in chromaticity values or intensities across the surface.

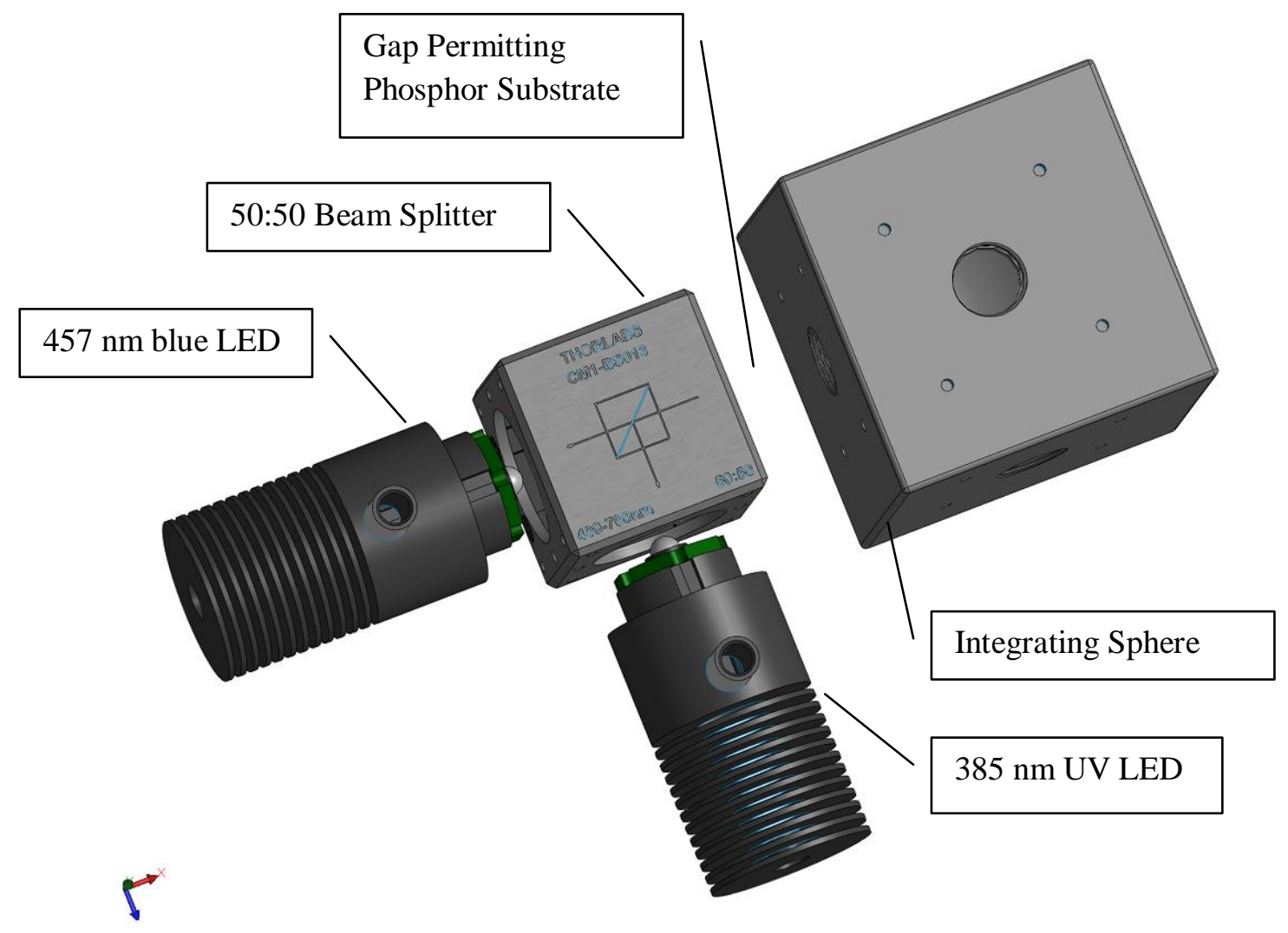

Figure 19 - The benefit of this set up is that both the light sources emerge at one end of the beamsplitter, each at $50 \%$ intensity, and interact with a phosphor substrate with minimal spatial displacement apparent between the two light sources. The integrating sphere collects all the light over a given area and directs it along a fiber optic cable, further reducing spatial displacement effects. 


\section{Computer Interface}

Microsoft Visual Basic 6.0 provided the ability to control the various devices during testing. A program was developed to interface to the Newport Linear Motion Controller as well as both DAQs (Figure 20). 'Home Search,' 'Position Read Out,' and 'Absolute Positioning' enable the linear stages to be homed, current positions to be read on the computer monitor, and movement to specific coordinates respectively. 'Individual Movement' allows for consecutive changes in position of any distance out to the motor limits. 'Sequential Movement' enables users to define a specific path and move to any position in that path based upon the location number of a given coordinate. 'LED Mapping' and 'Linear LED Mapping' were developed for automated control. The only difference between the two is that the later measures only in a one dimensional path while the other moves in a two dimensional squared region. Inputs are minimum to maximum positions for a given axis and the increment desired for movement. The majority of the tests were conducted between $-15.0 \mathrm{~mm}$ and $15.0 \mathrm{~mm}$ with $0.5 \mathrm{~mm}$ increments. During test execution the National Instruments DAQ is triggered to send a 5 $\mathrm{V}$ signal to the spectrometer resulting in a saved spectrum at the given position. The Agilent DAQ is cycled through each of the occupied channels (voltage, amperage, and temperature) and the current readings are sent to an Excel file. 'Time Dependence' performs in a similar method to the above two tests but instead of sending signals to the DAQs every time the stages move it does so based on a given time interval. 'Manual Control' enables a user to select 'Record Data' which triggers both DAQs at their own discretion. Both 'Time Dependence' and 'Manual Control' are designed for use with the 
beam splitter and integrating sphere since signals to the Linear Motion Controller are not sent.

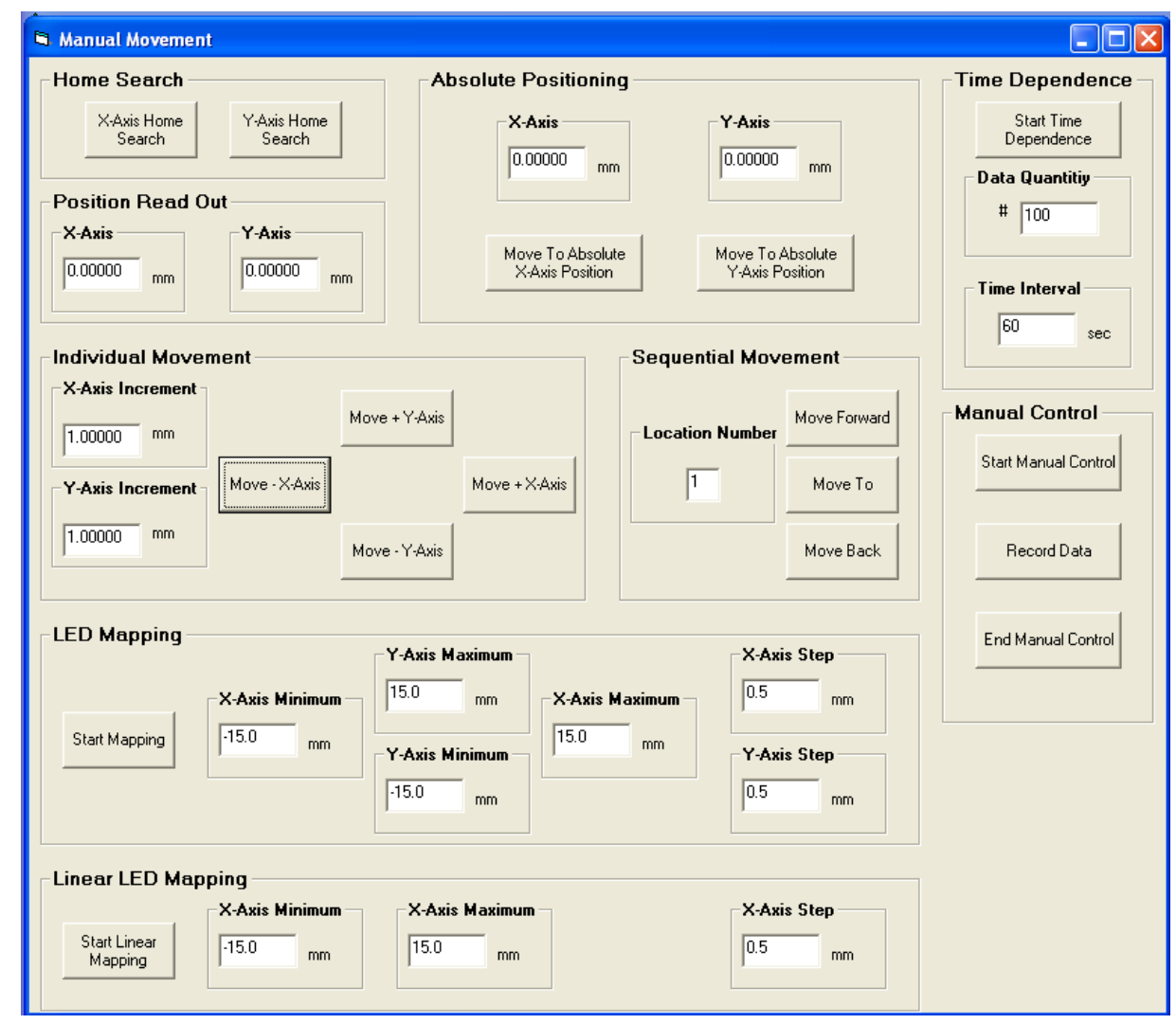

Figure 20 - Program menu enabling users to input numerics and select options necessary for testing. The majority of tests were conducted using the region titled 'LED Mapping' to characterize light emitted from the phosphor/PDMS substrates.

\section{Distance between the LEDs and the Phosphor Substrate}

The apparatus incorporates multiple points which increases the distance between the LEDs and the phosphor substrate. Throughout this study those distances were held as constant as possible to minimize their influence on intensity. An initial study determined that adjusting the distance between the phosphor substrate and the LED had negligible effect on chromaticity values. Another finding was that changing the distance between a 
phosphor substrate and the LED from 0 to $6 \mathrm{~mm}$ had a significant effect on the intensity of light observed. The intensity measurements of that study were essentially of light collected directly above the substrate. Therefore the study did not account for light dispersed in other directions besides that perpendicular to the substrates surface. Based on those results increasing the distance between the phosphors and the LED will decrease the performance of a light source in a directional application. The conclusion from that study recommended placing the phosphor as close as possible to the LED die without any significant increase in die temperature.

\section{Integrating Sphere Behavior}

The benefit with the integrating sphere is that one chromaticity point comes from each test, as opposed to the two dimensional array of chromaticity values seen when focusing on a specific spot. Integrating spheres operate by allowing light to enter a port and then scattering that light equally over the internal surface. Positioning data is essentially irrelevant in this process since only one spectrum is obtained from a region of light illuminating the sphere. The distance a phosphor layer is from the integrating sphere induces a different color value. For instance, the closer a light source is to the spheres' collection hole the more light from the light sources center influences the spectrum relative to the outside. As that same source moves farther away from the sphere's collection hole, light from the outside is more likely to illuminate the sphere. A source which has a large gradient of colors at its surface is more susceptible to this behavior. Tests performed with the integrating sphere insured a constant distance from the 
phosphor/PDMS substrate to the collection hole by utilizing a micrometer attached to a linear stage.

\section{Sample Preparation}

\section{PDMS Background}

PDMS is the most widely used silicon-based (inorganic) polymer ${ }_{(26)}$. It found its way into the microfabrication industry because of its ability to retain tight tolerances, without large forces applied, and the ease for which it is created (Figure 21).

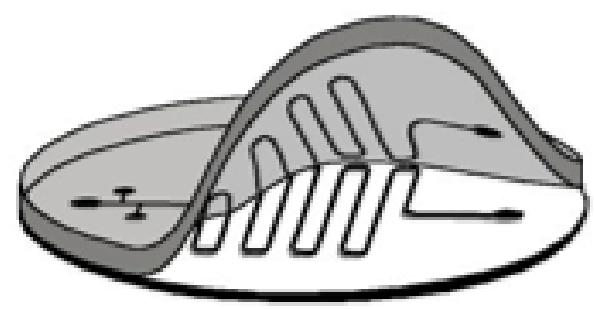

Figure 21 - Drawing depicting transparent PDMS easily being removed from a silicon wafer following a microfluidic processing technique. The silicon wafer contains a pattern for microfluidics and the PDMS mold contains the inverse of that pattern ${ }_{(27)}$.

It also appears in everyday products such as hair conditioners, silicones, silly putty, and contact lenses. The properties which make it appealing to the LED project is that it is optically clear (transparent to ultraviolet and visible light) along with being non-toxic.

Purpose of PDMS

In order to accurately and repeatedly position phosphor particles above the various LEDs used in testing, polydimethylsiloxane (PDMS) was used as a medium. It is manufactured by curing a viscous mixture of a given ratio of base and agent. Therefore the material has the ability to be molded in a desired shape for which it will maintain upon curing. Curing consists of a mild heat treatment, not necessary but will hasten the cure rate, for a given amount of time. Two methods of production were established to create the 
phosphor/PDMS substrates based on the thickness desired. For substrates thicker than 1 $\mathrm{mm}$ the "pouring" method was used. For substrates thinner than $1 \mathrm{~mm}$ a method involving spin coating was necessary to produce a uniform thickness. To adhere the final substrates to glass or pure PDMS two methods of plasma bonding were utilized. Refer to Appendix A for a complete procedure on how to prepare samples with "pouring," "spin coating," and "plasma bonding" techniques.

\section{Experimental Procedure}

The following section discusses the procedure for preparing the phosphor/PDMS samples for use in the testing apparatus, the software configuration steps to begin the experiment, and what is required to process the data for producing graphs and matrices for analysis. Plasma bond the one inch diameter phosphor/PDMS sample to a one inch diameter glass substrate to insure the sample is as flat and immobile as possible during testing. To plasma bond, follow the plasma gun procedure explained above under "Plasma Bonding." Accurately position the ring inside the one inch diameter cylinder which surrounds the LED. Insert the bonded pieces into a one inch diameter threaded cylinder with the phosphor/PDMS side facing down (against the ring). Place the cylinder over the LED and begin preparing the apparatus with the procedure provided in Appendix B. At the completion of the test the spectrometer files need to be converted to Equi distance values from 380 to $770 \mathrm{~nm}$ in $10 \mathrm{~nm}$ increments. Upon conversion the files may be uploaded and analyzed using Excel Macros in a manner described in Appendix C. 


\section{CHAPTER V: OBSERVED BEHAVIORS USING A 457 nm THORLABS BLUE LED AND YAG:Ce PHOSPHOR}

\section{Introduction}

The purpose of this thesis is to determine the relationship between phosphors and their ability to produce specific values of white light. The ultimate goal would be to mimic a black body radiator between 2500 and 7500 Kelvin. To accomplish this task, equations were developed for multiple variables which influence the light output. The following section provides the trends found using a blue high-brightness Thorlabs LED and cerium doped yttrium aluminum garnet (YAG:Ce). YAG:Ce phosphor was selected to take maximum advantage of the blue light emitted from a Thorlabs LED (Appendix D and E). YAG:Ce most efficiently absorbs light in the blue region (peak of $457 \mathrm{~nm}$ ) and reemits light in the yellow region (peak of $562 \mathrm{~nm}$ ). When in combination with blue light, YAG:Ce has the ability to produce an apparent white light to an observer. By mixing phosphor and PDMS together, a flexible material is created which photoluminesces with blue light and can be accurately positioned above the light source.

\section{Crystallography and Photoluminescence of YAG:Ce}

YAG:Ce is a synthetic material with a complex unit cell which repeats in a crystalline manner. The material is of the garnet group which, in natural form, is commonly found as gemstones (28). The chemical formula for YAG:Ce is commonly reported as $\mathrm{Y}_{3} \mathrm{Al}_{5} \mathrm{O}_{12}: 0.05 \mathrm{Ce}$ where the ratio of elements are provided with the subscripts and cerium exists in 0.05 atomic percent of the lattice. The actual unit cell is comprised of 160 atoms and can be described as a combination of octahedrons, tetrahedrons, and dodecahedrons 
(Figure 22). Oxygen atoms link these structures together as each one is a member of two dodecahedrons, one octahedron, and one tetrahedron ${ }_{(29)}$. The relatively small quantities of cerium atoms occupy yttrium locations within the host lattice.

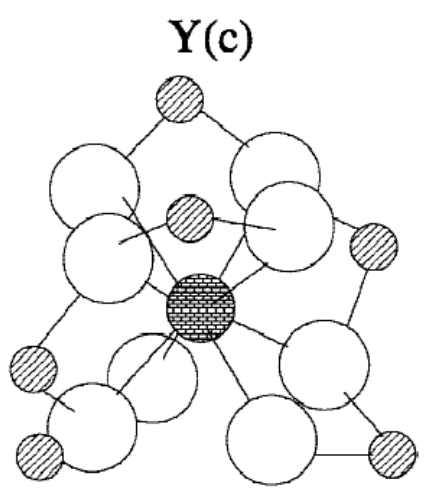

(A)

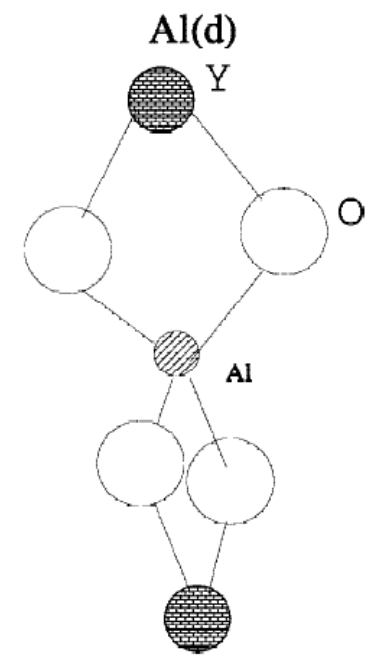

(B)

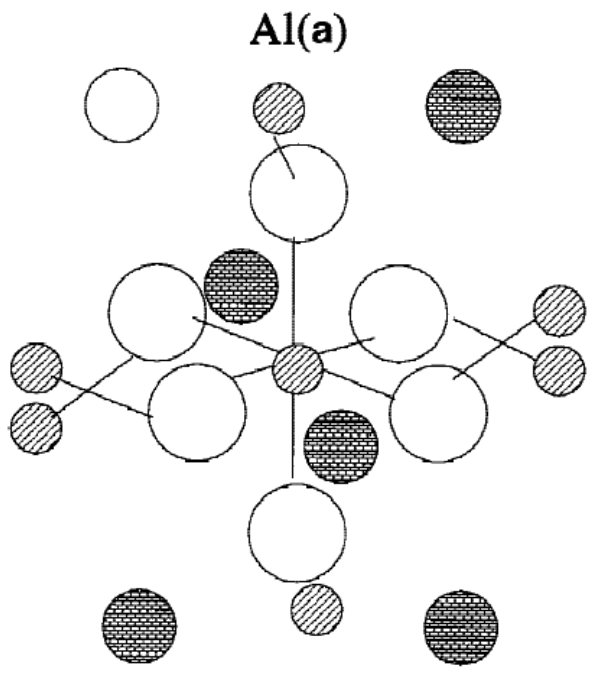

(C)

Figure 22 - The arrangement of atoms within the YAG crystal structure. Each unit cell is comprised of a combination of a dodecahedrally coordinated yttrium atom (A), a tetrahedrally coordinated aluminum atom (B), and an octahedrally coordinated aluminum atom (C). Largest spheres (white) represent oxygen atoms, smallest spheres (light gray) represent aluminum atoms, and medium sized spheres (dark gray) represent yttrium atoms $(29)$.

Cerium atoms (like some other rare earth ions) may produce broad band emission when an excited electron returns from a $5 \mathrm{~d}$ orbital to the $4 \mathrm{f}$ orbital. This is apparent in trivalent ions such as $\mathrm{Ce}^{3+}, \mathrm{Pr}^{3+}, \mathrm{Nd}^{3+}$. Their excited configuration is $5 \mathrm{~d}^{1}$ and the ground state is $4 \mathrm{f}^{1}$. This ground state enables two levels separated by spin-orbit coupling $\left({ }^{2} \mathrm{~F}_{5 / 2}\right.$ and ${ }^{2} \mathrm{~F}_{7 / 2}$ ). While the excited configuration is split into 2 to 5 levels, the emission occurs from a decay of the lowest $5 \mathrm{~d}^{1}$ configuration to the two ground state levels. This accounts for the increased width, created by a superposition of a double-band shape, typical of emission spectrums with a $\mathrm{Ce}^{3+}$ doped phosphor. The emission is also intense since the 
$5 \mathrm{~d}$ to $4 \mathrm{f}$ transition is parity allowed and spin selection does not apply. The decay time in the instance of YAG:Ce is 70 nanoseconds. This is slightly longer than other materials with $\mathrm{Ce}^{3+}$ because the emission occurs at a higher wavelength. Decay time is proportional to the square of the emission wavelength. The longer decay time stems from a crystal-field effect (two ground state levels) from the YAG:Ce lattice. Stokes shift is small for $\mathrm{Ce}^{3+}$ due to the nephelauxetic effect (an expanded d-orbital electron cloud) which reduces the energy difference between $4 \mathrm{f}^{1}$ and $5 \mathrm{~d}^{1}$ configurations. Another factor which reduces stokes shift is the crystal field splitting of the $5 \mathrm{~d}^{1}$ configuration. In this instance a large low-symmetry crystal field will lower the lowest crystal-field component at the emission origin. Meaning that the excited level for which an electron decays from has a lower energy then what would be observed in a symmetrical crystal field, thus producing wavelengths of light near $550 \mathrm{~nm}_{(24)}$.

\section{DOE: Height, Concentration, Thickness}

Introducing phosphor into PDMS allows for four major variables to be studied (Figure 23). The first variable considered is concentration. Concentration is measured as a percentage of the weight of phosphor added to the weight of PDMS and labeled throughout this document as "wt\%". The second variable which influences the color and intensity is the thickness of the phosphor/PDMS substrate. Both the pouring and spincoating method allow for control of the thickness which translates to more or less phosphor directly above the LED. Adhering the phosphor/PDMS substrate to a glass disc enables the substrate to be rigidly held above the LED die. Features of the testing apparatus allow for the adjustment of the distance from the rigid substrate to the LED die 
(referred to as 'Height') which has been found to influence the intensity output. A fourth variable which will be discussed in Chapter VI is amperage applied to the LEDs. The results reported in this document are from a blue LED with constant amperage $(0.67 \mathrm{~A})$. It is important to note that throughout this DOE the LED die was fixed below the center of the one inch diameter phosphor/PDMS substrate. Measurements "directly above LED die" indicate what is observed at the center (radius $=0 \mathrm{~mm}$ ) of the phosphor/PDMS substrate. Measurements at radii greater than $0 \mathrm{~mm}$ were obtained by moving the entire assembly (LED die with fixed phosphor/PDMS substrate) so that the optics tube was focused at some position denoted by the linear motion controller.
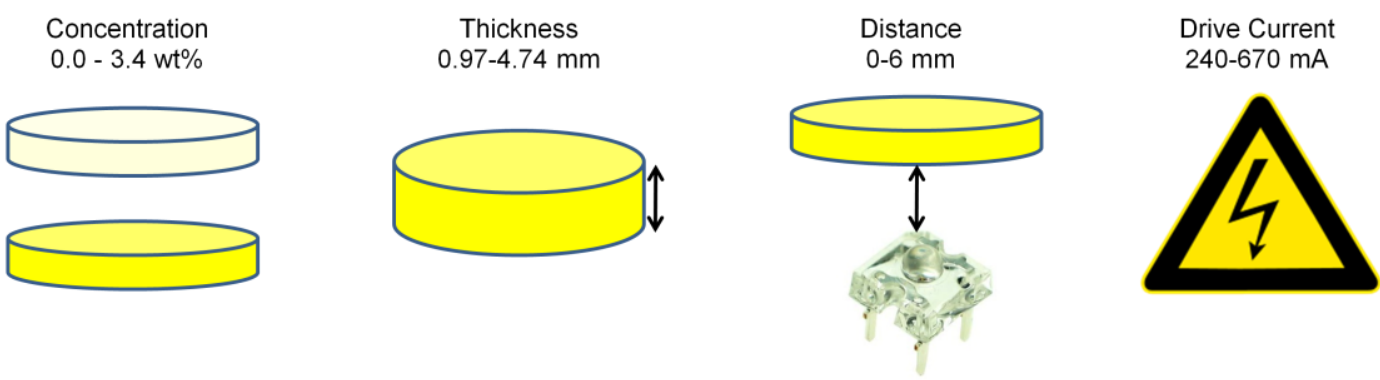

Figure 23 - Four variables, three of which were tested with a $457 \mathrm{~nm}$ Thorlabs LED held at constant amperage, that influence the observed color of light emitted from an LED.

Tested Samples

Initial testing was conducted to determine the influence of phosphor concentration, substrate thickness, and substrate distance from the LED die on the color of light produced. The equations for chromaticity color values apply to a blue (457 nm) Thorlabs LED held at the maximum amperage (approximately $670 \mathrm{~mA}$ ) with their recommended power supply. Each phosphor/PDMS substrate was one inch $(25.4 \mathrm{~mm})$ in diameter. 


\section{Concentration}

Five different weight percents were created for concentration testing. Their thickness was kept as consistent as the pouring method would enable. Average thickness was 2.49 $\mathrm{mm}$ with a standard deviation of $0.06 \mathrm{~mm}$. Concentration ranged from $1.8 \mathrm{wt} \%$ to 3.4 wt\%, as listed in Table II, which was adequate to provide a color range from blue to white in the center of the phosphor/PDMS substrate.

\section{Table II - Concentration Test}

\begin{tabular}{|l|l|l|l|}
\hline $\begin{array}{l}\text { Concentration } \\
(\mathbf{w t} \%)\end{array}$ & $\begin{array}{l}\text { Thickness } \\
(\mathbf{m m})\end{array}$ & $\begin{array}{l}\text { x-chromaticity directly } \\
\text { above LED die }\end{array}$ & $\begin{array}{l}\text { y-chromaticity directly } \\
\text { above LED die }\end{array}$ \\
\hline 1.8 & 2.50 & 0.162 & 0.088 \\
2.2 & 2.41 & 0.169 & 0.103 \\
2.6 & 2.43 & 0.220 & 0.208 \\
3.0 & 2.55 & 0.216 & 0.198 \\
3.4 & 2.54 & 0.230 & 0.226 \\
\hline
\end{tabular}

Thickness

Six different thicknesses were produced from the same batch of a $3.8 \mathrm{wt} \%$ mixture. Because the substrates were produced from the same batch no standard deviation can be applied to the average $3.8 \mathrm{wt} \%$ value. It is assumed that each substrate had the same concentration. The target thicknesses were between 1 and $4.75 \mathrm{~mm}$ with $0.75 \mathrm{~mm}$ increments. However, variance in the pouring method produced slightly different values, as shown in Table III. 
Table III - Thickness Test

\begin{tabular}{|l|l|l|l|}
\hline $\begin{array}{l}\text { Thickness } \\
(\mathbf{m m})\end{array}$ & $\begin{array}{l}\text { Concentration } \\
(\mathbf{w t} \%)\end{array}$ & $\begin{array}{l}\text { x-chromaticity directly } \\
\text { above LED die }\end{array}$ & $\begin{array}{l}\text { y-chromaticity directly } \\
\text { above LED die }\end{array}$ \\
\hline 0.97 & 3.8 & 0.152 & 0.068 \\
1.79 & 3.8 & 0.191 & 0.149 \\
2.21 & 3.8 & 0.229 & 0.224 \\
3.20 & 3.8 & 0.295 & 0.352 \\
3.70 & 3.8 & 0.314 & 0.387 \\
4.74 & 3.8 & 0.369 & 0.479 \\
\hline
\end{tabular}

Distance

An internally threaded cylinder was utilized to accurately position the $1.79 \mathrm{~mm}$ thick 3.8 wt\% sample above the Thorlabs LED. Table IV lists the six positions tested ranging from 1 to $6 \mathrm{~mm}$ above the die. Measurements were taken with a veneer caliper from the top of the cylinder (one defined location) to the ring depth. The focal point of the optics tube assembly was raised and lowered accordingly using a micrometer attached to a linear stage.

Table IV - Distance Test

\begin{tabular}{|l|l|l|}
\hline $\begin{array}{l}\text { Height } \\
\text { (mm) }\end{array}$ & $\begin{array}{l}\text { x-chromaticity directly } \\
\text { above LED die }\end{array}$ & $\begin{array}{l}\text { y-chromaticity directly } \\
\text { above LED die }\end{array}$ \\
\hline 1 & 0.206 & 0.177 \\
2 & 0.202 & 0.170 \\
3 & 0.203 & 0.171 \\
4 & 0.198 & 0.159 \\
5 & 0.202 & 0.168 \\
6 & 0.199 & 0.159 \\
\hline
\end{tabular}




\section{Data Analysis and Results}

To analyze the data it is useful to divide the visible spectrum into two parts for which the intensity is integrated over. The range from 380 to $510 \mathrm{~nm}$ is used to describe the intensity of the blue LED. The range from 510 to $770 \mathrm{~nm}$ describes the degree of photoluminescence from the phosphor/PDMS substrate. The overall range of 380 to 770 $\mathrm{nm}$ describes the intensity of visible light which the observer would see.

Intensity Profiles

To describe the quantity of light emitted from a given radii along the one inch diameter phosphor/PDMS substrate, intensity profiles were created displaying a cross section at y $=0$ (Figures 24, 25, and 26). A dramatic change in intensity is found when comparing the $3.8 \mathrm{wt} \%$ phosphor substrates from 0.97 to $4.74 \mathrm{~mm}$ thick (Figure 24). Toward the outer edges of the substrate, intensity values begin to converge on each other but in a region within $2 \mathrm{~mm}$ from the LED die there is a substantial difference between the various thicknesses. The $0.97 \mathrm{~mm}$ thick sample was capable of transmitting the greatest amount of photons directly above the die. The intensity decreases as thickness increases up to the thickest tested sample of $4.74 \mathrm{~mm}$. However, when chromaticity values are examined it's found that the $0.97 \mathrm{~mm}$ thick sample with $3.8 \mathrm{wt} \%$ phosphor is not capable of producing white light above the LED. There is simply not enough photoluminescence to create white light because of the lack of phosphor with a thin and low concentration substrate. 


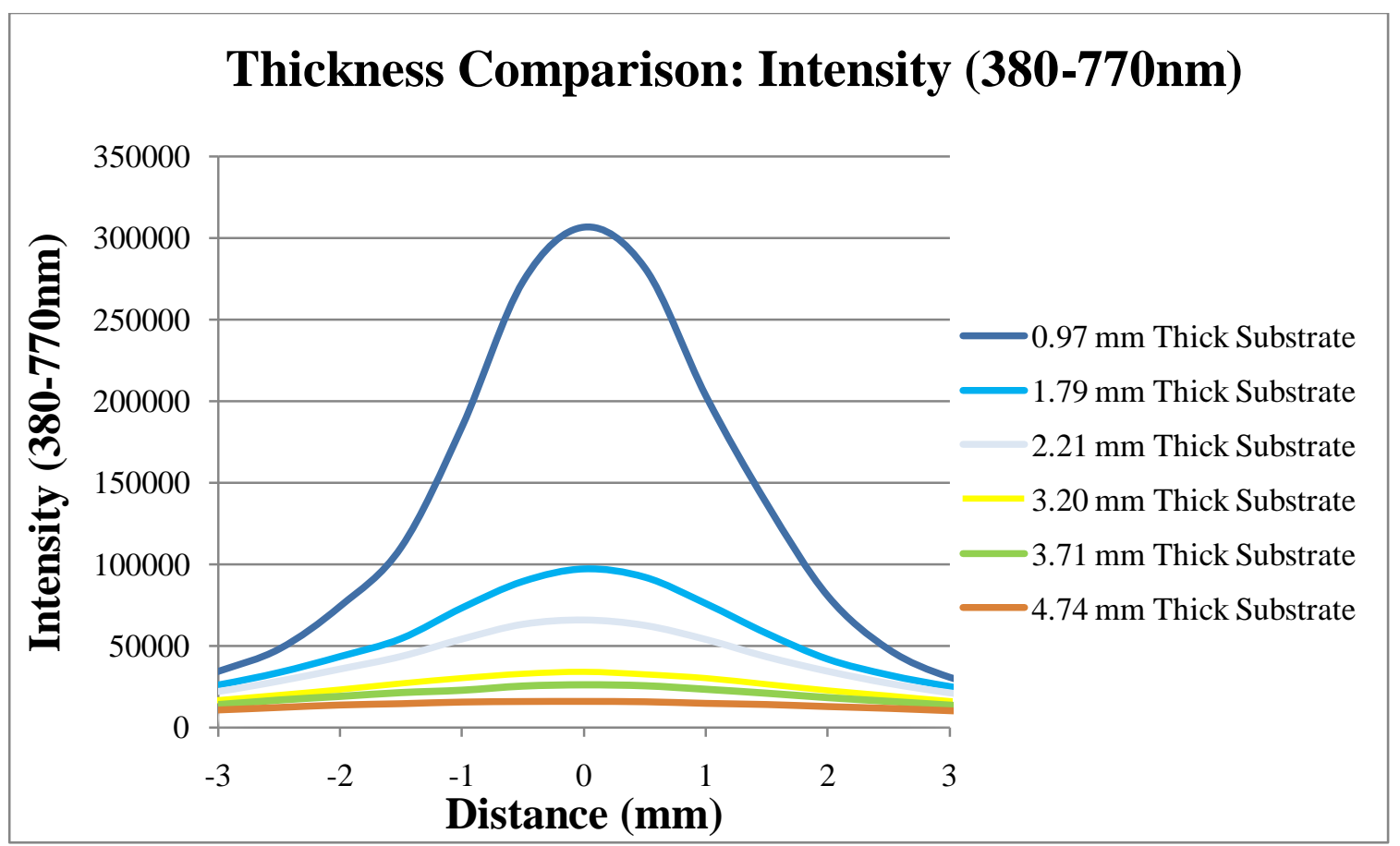

Figure 24 - Displaying the total intensity of the visible spectrum at a given location over the crosssection of various thicknesses of $3.8 \mathrm{wt} \%$ phosphor/PDMS substrates passing directly over the LED die.

When comparing concentration samples the range between intensity values is not as extreme as what was seen in the thickness tests (Appendix F, G, and H). However the difference between intensity at a given point through PDMS without phosphor compared to samples with any amount of phosphor is substantial. PDMS without phosphor is capable of transmitting nearly ten times the amount of light directly above the LED die compared to when phosphor is introduced (Figure 25). 


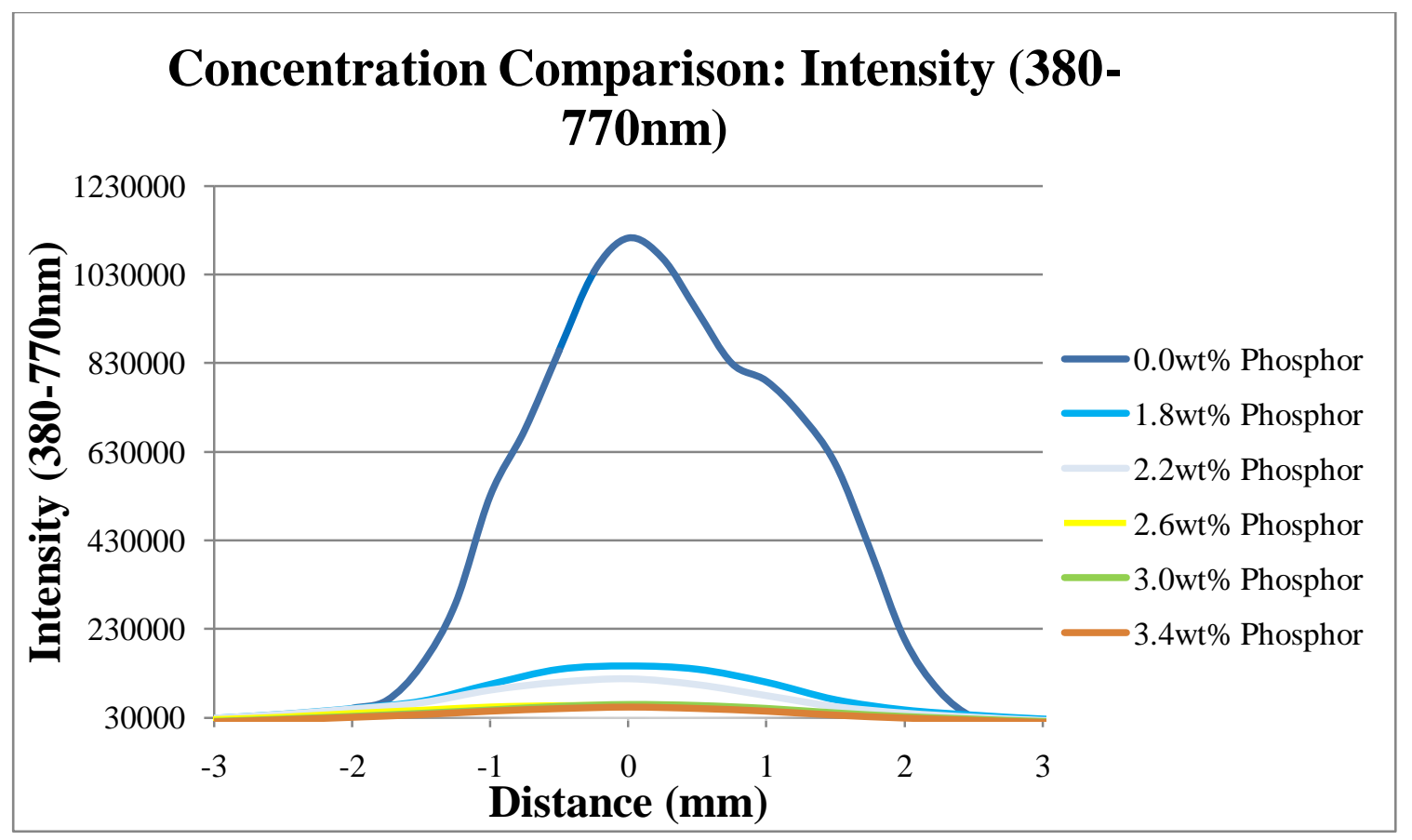

Figure 25 - Intensity comparisons between the six different, $2.5 \mathrm{~mm}$ thick, phosphor wt\% samples. Intensity directly above the LED is substantially lower when phosphor is used due to its ability to diffuse light.

This occurs partly because of the phosphors ability to diffuse light. It is important to note that this intensity curve represents a cross section which passes directly over the die. It does not account for the total intensity count above the one inch diameter substrate.

Differences in light intensity over a $1.79 \mathrm{~mm}$ thick $3.8 \mathrm{wt} \%$ phosphor/PDMS substrate varied significantly between 1 and $6 \mathrm{~mm}$ distances (Figure 26). 


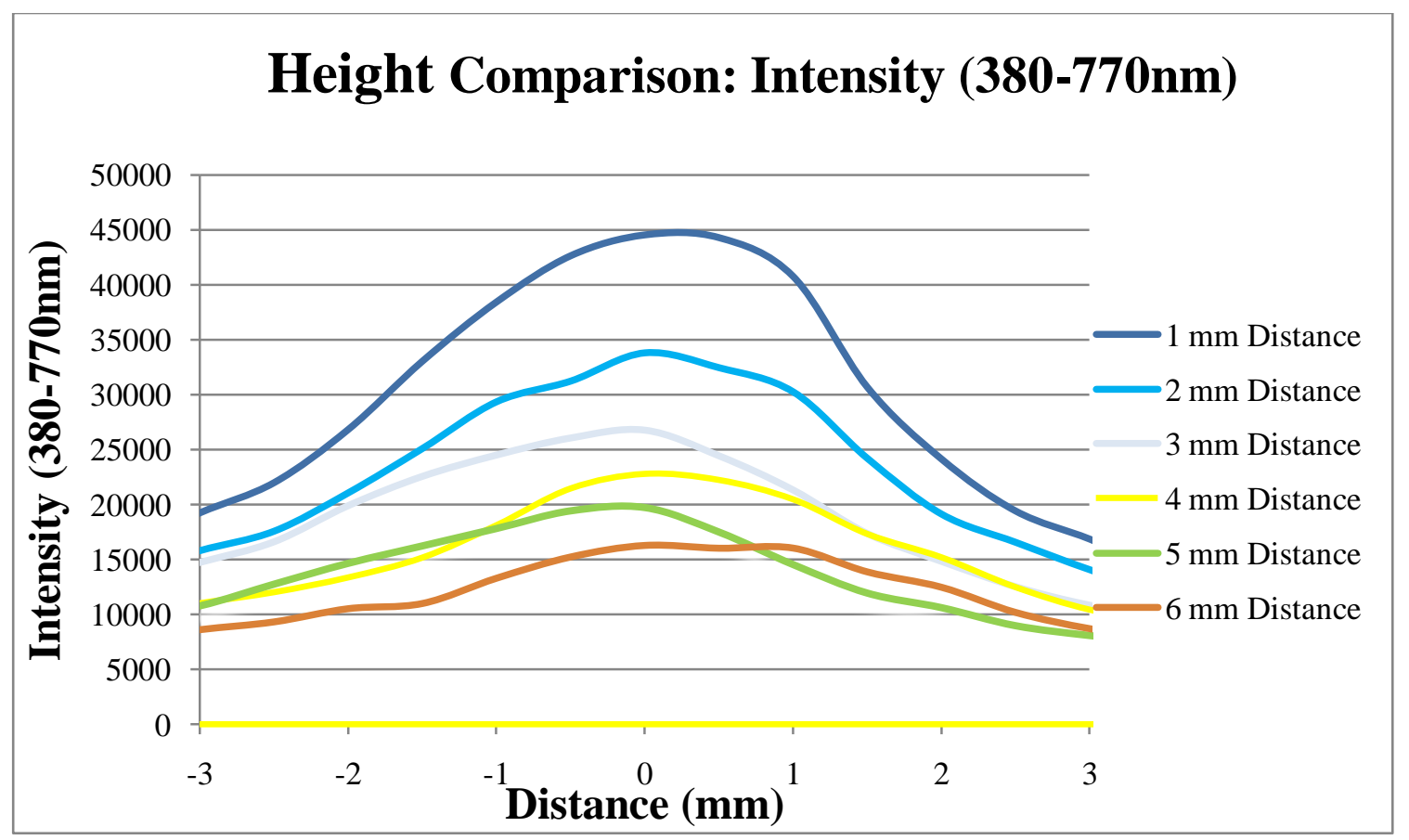

Figure 26 - The height measurements shown in this graph are measured from the top of the epoxy covering the 16 LED dies to the bottom of the phosphor substrate. Each height measurement was made with the $1.79 \mathrm{~mm}$ thick, $3.8 \mathrm{wt} \%$ sample.

The closer the substrate is to the LED die, the greater the intensity of light over the entire phosphor/PDMS substrate in the one inch cylinder. The significance of the light intensity for a given distance is amplified when we consider the entire surface as shown in the following tables.

Intensity profiles have the distribution characteristic of greatest intensity in the center of the phosphor/PDMS substrate and decreasing intensity at greater radii for three major reasons. Light emitted from the LED has a relatively small divergence angle for the given set up meaning that light is mainly focused on the center of the phosphor/PDMS substrate and does not have sufficient distance to illuminate the entire one inch substrate with sufficient intensity. The second reason pertains to the testing apparatus and the 
nature of the lens only collecting light which is emitted perpendicular to the substrate. The transparency of PDMS does enable the lens to collect light emitted from the surface in directions other than perpendicular but these angles are small deviations from normal and come from phosphor particles beneath the surface. This is one reason which limits the spot size capability of the testing apparatus. The third reason stems from the diffusing capabilities of phosphor particles. If we ignore the photoluminescent process and only consider incident light which bypasses a particle or reflects off a particle we may begin to visualize a chain reaction. It begins with a particle directly above the LED deflecting incident light at an angle other than parallel to its incident angle. Light is then directed to another particle where it may also be deflected in a manner which directs it to other particles in the substrate rather than through the substrate. This process enables light to reach the edges of the substrate despite the small divergence angle of the LED. Reduced intensity at greater radii stems from the increase of phosphor particles light must pass through at those distances relative to a path directly through the center of the phosphor/PDMS substrate. This intensity variance is partially addressed by creating a substrate which contains more equivalent light paths in all directions. Meaning that regardless of direction there is a similar amount of phosphor particles light emitted from the LED must encounter to pass through the lens. A Gaussian lens partly addresses this issue and is discussed in detail in Chapter VIII.

The following tables display the total counts measured over a 15 x $15 \mathrm{~mm}$ region centered above the LED die. Counts have been normalized according to the integration 
time used during the testing. Percent intensity values are with respect to the test which produced the greatest amount of counts.

Thickness tests displayed an interesting trend where the thicker samples not only decreased the amount of light above the substrate but also reduced the amount of photoluminescence as shown in Table V. The $2.21 \mathrm{~mm}$ thick substrate displayed the most photoluminescence for the blue Thorlabs LED at maximum current. Overall intensity displayed a major change with maximum intensity corresponding to the thinnest sample and rapidly decreasing with increasing thickness.

Table V - Thickness Comparison (Adjusted Total Intensity)

\begin{tabular}{|c|c|c|c|c|c|c|}
\hline $\begin{array}{l}\text { Thickness } \\
(\mathrm{mm})\end{array}$ & $\begin{array}{l}\text { Counts } \\
(380-510 \\
\mathrm{nm})\end{array}$ & $\begin{array}{l}\text { Counts } \\
(520-770 \\
n m)\end{array}$ & $\begin{array}{l}\text { Counts } \\
(380-770 \\
n m)\end{array}$ & $\begin{array}{l}\% \text { Intensity } \\
(380-510 \\
\mathrm{nm})\end{array}$ & $\begin{array}{l}\% \text { Intensity } \\
(510-770 \\
n m)\end{array}$ & $\begin{array}{l}\% \text { Intensity } \\
(380-770 \\
n m)\end{array}$ \\
\hline 0.97 & $1.45 \mathrm{E}+07$ & $5.97 \mathrm{E}+06$ & $2.05 \mathrm{E}+07$ & $100 \%$ & $85 \%$ & $100 \%$ \\
\hline 1.79 & $5.89 \mathrm{E}+06$ & $6.76 \mathrm{E}+06$ & $1.27 \mathrm{E}+07$ & $41 \%$ & $96 \%$ & $62 \%$ \\
\hline 2.21 & $3.53 \mathrm{E}+06$ & $7.03 \mathrm{E}+06$ & $1.06 \mathrm{E}+07$ & $24 \%$ & $100 \%$ & $52 \%$ \\
\hline 3.20 & $1.54 \mathrm{E}+06$ & $6.57 \mathrm{E}+06$ & $8.11 \mathrm{E}+06$ & $11 \%$ & $93 \%$ & $40 \%$ \\
\hline 3.71 & $1.06 \mathrm{E}+06$ & $6.22 \mathrm{E}+06$ & $7.28 \mathrm{E}+06$ & $7 \%$ & $89 \%$ & $36 \%$ \\
\hline 4.74 & $4.63 \mathrm{E}+05$ & $5.49 \mathrm{E}+06$ & $5.96 \mathrm{E}+06$ & $3 \%$ & $78 \%$ & $29 \%$ \\
\hline
\end{tabular}

Table VI indicates that concentration also contributed to overall intensity with the trend that increasing concentration decreased the total intensity. During this study $3.4 \mathrm{wt} \%$ produced the closest spectrum to white light directly above the LED die. However a concentration of that thickness permitted only half the amount of light as the blue LED alone. 
Table VI - Concentration Comparison (Adjusted Total Intensity)

\begin{tabular}{|c|c|c|c|c|c|c|}
\hline $\begin{array}{l}\text { Concentration } \\
(\mathrm{wt} \%)\end{array}$ & $\begin{array}{l}\text { Counts } \\
(380-510 \\
\mathrm{nm})\end{array}$ & $\begin{array}{l}\text { Counts } \\
(520-770 \\
\mathrm{nm})\end{array}$ & $\begin{array}{l}\text { Counts } \\
(380-770 \\
\mathrm{nm})\end{array}$ & $\begin{array}{l}\% \\
\text { Intensity } \\
(380-510 \\
\mathrm{nm})\end{array}$ & $\begin{array}{l}\text { \% } \\
\text { Intensity } \\
(510-770 \\
\mathrm{nm})\end{array}$ & $\begin{array}{l}\% \\
\text { Intensity } \\
(380-770 \\
\mathrm{nm})\end{array}$ \\
\hline 0.0 & $2.94 \mathrm{E}+07$ & $-9.55 E+06$ & $1.99 \mathrm{E}+07$ & $100 \%$ & $-146 \%$ & $100 \%$ \\
\hline 1.8 & $9.59 \mathrm{E}+06$ & $5.50 \mathrm{E}+06$ & $1.51 \mathrm{E}+07$ & $33 \%$ & $84 \%$ & $76 \%$ \\
\hline 2.2 & $7.83 \mathrm{E}+06$ & $6.12 \mathrm{E}+06$ & $1.40 \mathrm{E}+07$ & $27 \%$ & $94 \%$ & $70 \%$ \\
\hline 2.6 & $5.79 E+06$ & $6.15 \mathrm{E}+06$ & $1.19 \mathrm{E}+07$ & $20 \%$ & $94 \%$ & $60 \%$ \\
\hline 3.0 & $4.10 \mathrm{E}+06$ & $6.29 \mathrm{E}+06$ & $1.04 \mathrm{E}+07$ & $14 \%$ & $96 \%$ & $52 \%$ \\
\hline 3.4 & $3.32 \mathrm{E}+06$ & $6.53 \mathrm{E}+06$ & $9.85 \mathrm{E}+06$ & $11 \%$ & $100 \%$ & $50 \%$ \\
\hline
\end{tabular}

Adjusting the height played a minimal role in changing chromaticity values but it greatly influenced total intensity. Table VII shows that as the phosphor/PDMS substrate was moved away from the LED die the intensity rapidly reduced. Moving the substrate $5 \mathrm{~mm}$ reduced the intensity by $98 \%$.

Table VII - Height Comparison (Adjusted Total Intensity)

\begin{tabular}{|l|lll|lll|}
\hline $\begin{array}{l}\text { Height } \\
(\mathrm{mm})\end{array}$ & $\begin{array}{llll}\text { Counts } \\
(380-510\end{array}$ & $\begin{array}{l}\text { Counts } \\
(520-770\end{array}$ & $\begin{array}{l}\text { Counts } \\
(380-770\end{array}$ & $\begin{array}{l}\text { \% Intensity } \\
(380-510 \\
\mathrm{nm})\end{array}$ & $\begin{array}{l}\text { \% Intensity } \\
(510-770 \\
\mathrm{nm})\end{array}$ & $\begin{array}{l}\text { \% Intensity } \\
(380-770 \\
\mathrm{nm})\end{array}$ \\
\hline 1 & $2.16 \mathrm{E}+08$ & $2.58 \mathrm{E}+08$ & $4.74 \mathrm{E}+08$ & $100 \%$ & $100 \%$ & $100 \%$ \\
2 & $2.48 \mathrm{E}+07$ & $2.79 \mathrm{E}+07$ & $5.28 \mathrm{E}+07$ & $12 \%$ & $11 \%$ & $11 \%$ \\
3 & $2.38 \mathrm{E}+07$ & $2.65 \mathrm{E}+07$ & $5.03 \mathrm{E}+07$ & $11 \%$ & $10 \%$ & $11 \%$ \\
4 & $9.97 \mathrm{E}+06$ & $1.05 \mathrm{E}+07$ & $2.05 \mathrm{E}+07$ & $5 \%$ & $4 \%$ & $4 \%$ \\
5 & $9.36 \mathrm{E}+06$ & $9.55 \mathrm{E}+06$ & $1.89 \mathrm{E}+07$ & $4 \%$ & $4 \%$ & $4 \%$ \\
6 & $4.42 \mathrm{E}+06$ & $4.55 \mathrm{E}+06$ & $8.96 \mathrm{E}+06$ & $2 \%$ & $2 \%$ & $2 \%$ \\
\hline
\end{tabular}

Based on intensity, it is important to create a phosphor/PDMS substrate with a minimal thickness and concentration while placing it as close as possible to the LED die. 


\section{Chromaticity Plots}

The following data displays two kinds of graphs to relay color information to the reader.

Using the data discussed previously, chromaticity values were calculated to understand each variables effect on color output. Each point on the following graphs represents the color seen directly above the LED for a given test. The thickness test displayed a wide range of colors directly above the LED (Figure 27). The $0.97 \mathrm{~mm}$ thick substrate had a minimal affect on the color output and appeared nearly as blue as the LED die by itself in the center. As we increase the thickness, which effectively increases the photoluminescence, we find a trend of lighter blues followed by various versions of white light and finally a yellow-green color. The $4.74 \mathrm{~mm}$ thick substrate produced a yellowish color directly above the LED die which indicates that it was too thick for the $3.8 \mathrm{wt} \%$ concentration used.

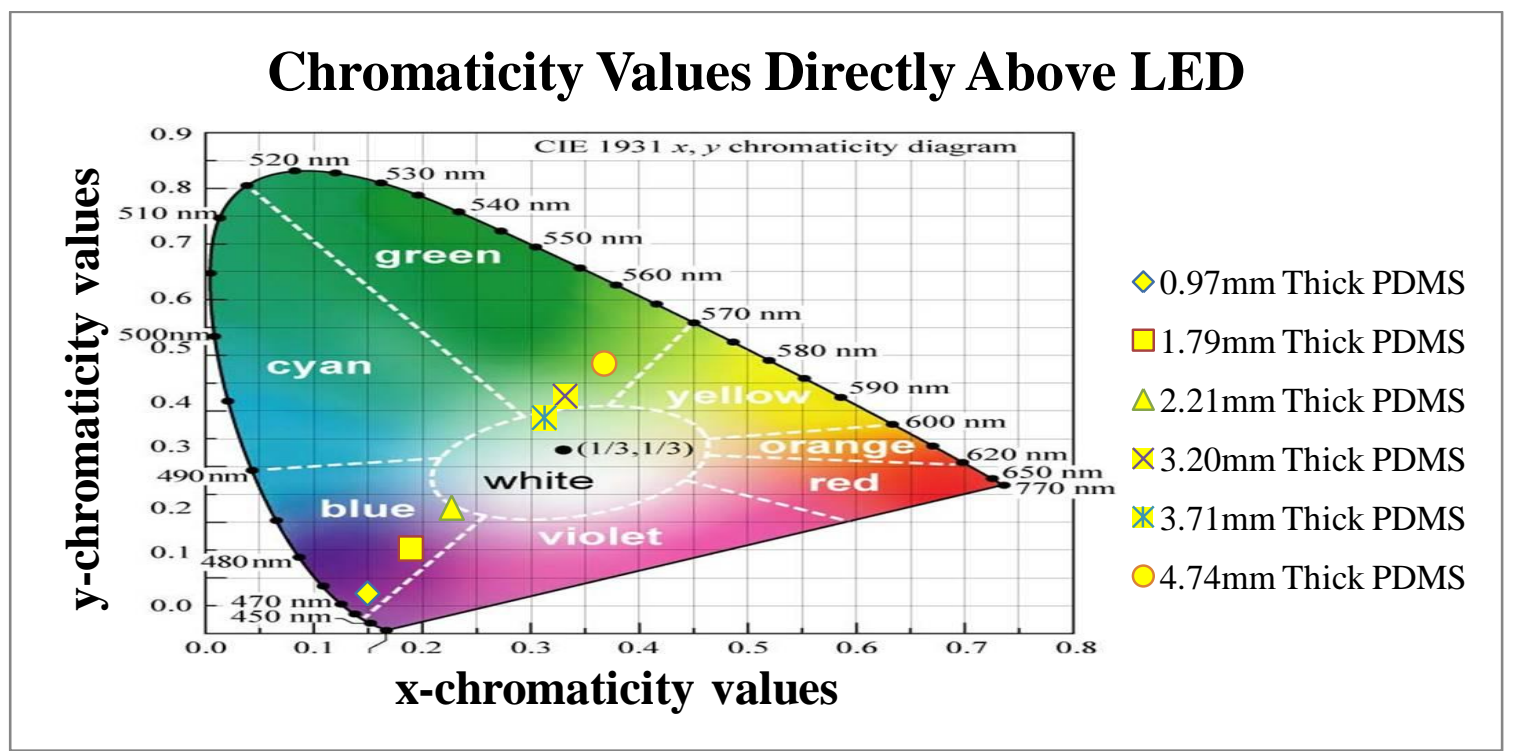

Figure 27 - The various colors created while looking directly above the LED die using different thickness phosphor/PDMS substrates. Height was maintained at $0 \mathrm{~mm}$ (contact with the LED's epoxy) and each sample has a phosphor concentration of $3.8 \mathrm{wt} \%$. 
Adjusting the concentration did not have as dramatic of an effect on the color directly above the LED as seen with the thickness tests (Figure 28). Ranging from 1.8 wt\% to 3.4 wt $\%$ brought the spectrum from a blue to a bluish-white color. Concentration would need to be increased in $2.5 \mathrm{~mm}$ thick substrates to obtain a white color directly above the LED die.

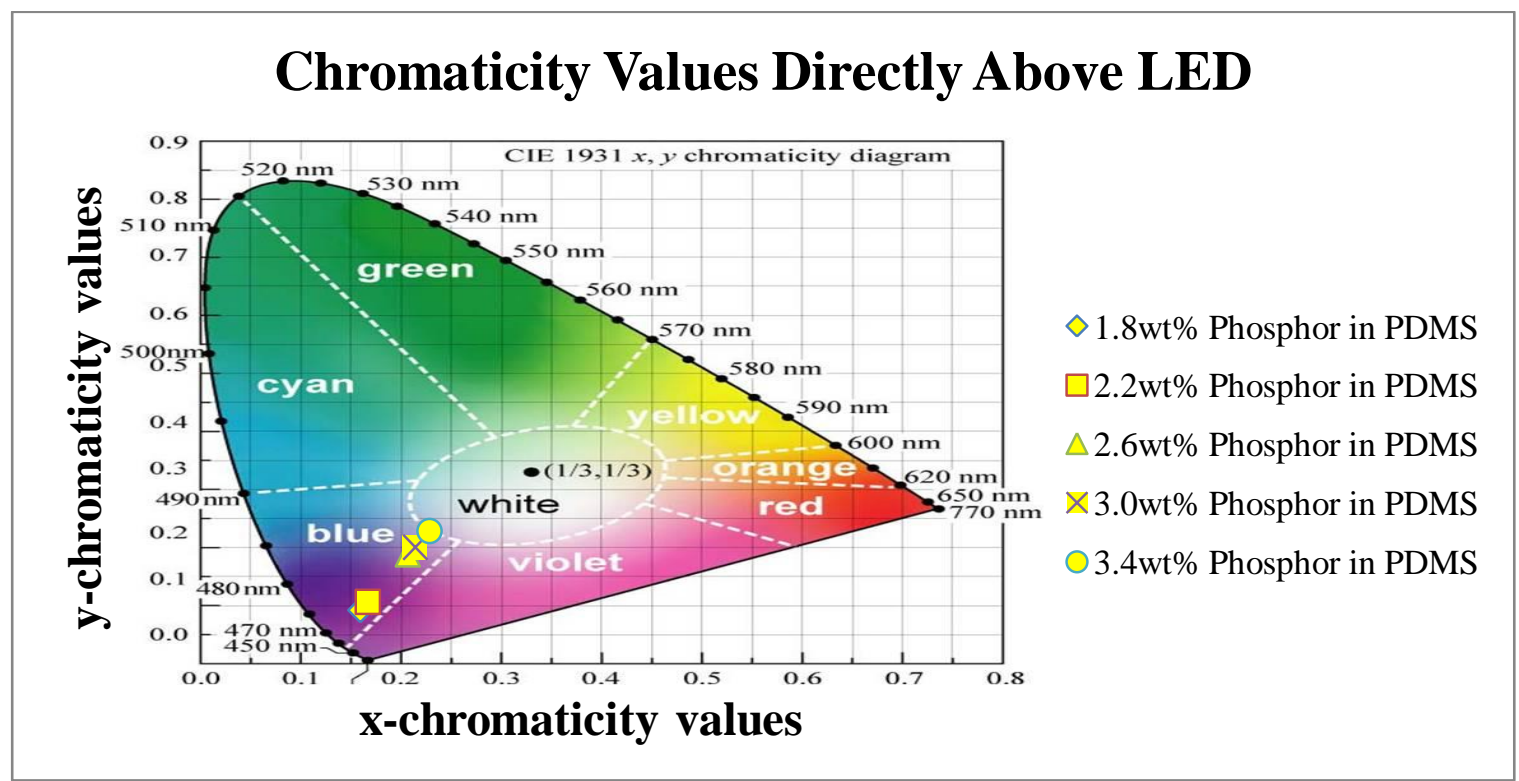

Figure 28 - Chromaticity values at a single point directly above the LED die. The $3.4 \mathrm{wt} \%$ sample is still too blue considering the values of white light pursued in this project. All experiments were conducted using $2.5 \mathrm{~mm}$ thick samples and a height of $0 \mathrm{~mm}$.

Height contributed very little to the color value (Figure 29). However there is still a trend where the greater the distance from the LED die the more blue the substrate appears. 


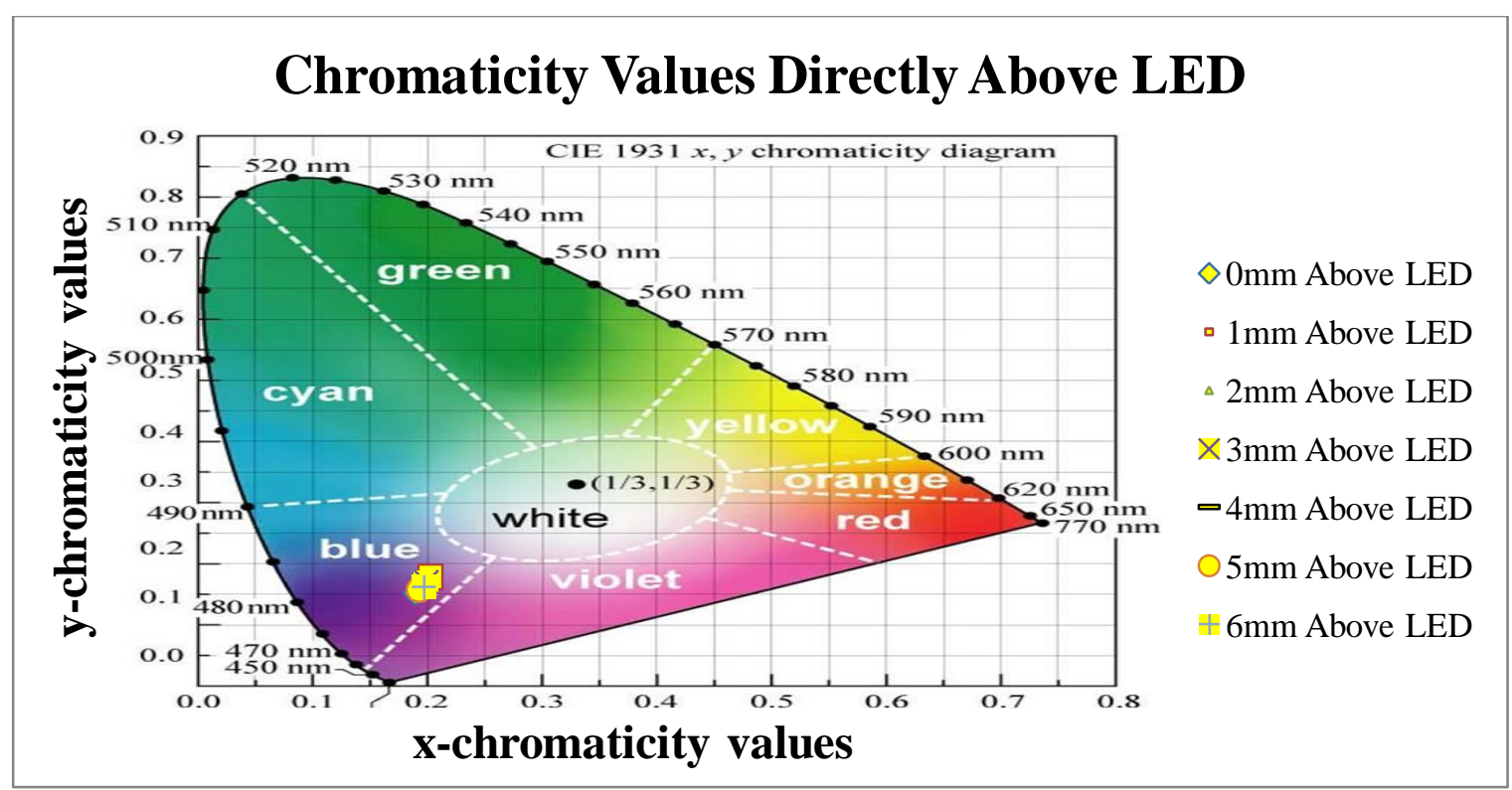

Figure 29 - Height tests had a minimal effect on chromaticity values. There is a trend of decreasing chromaticity values with increasing height but it is negligible in comparison to the variance we see in thickness and concentration tests. All height tests were conducted with the $2.5 \mathrm{~mm}$ thick, $3.8 \mathrm{wt} \%$ sample.

Chromaticity Graphs

A three dimensional plot of chromaticity values over the surface produces an image which displays the color seen above a given phosphor/PDMS sample (Figure 30). These graphs are good for relaying visual information but make it difficult to use for analysis purposes. For an analysis it is best to look at a cross-section of the plot (specifically at y $=0)$. 


\section{0 wt\% Phosphor in PDMS above 457 nm LED: $\mathrm{y}$ - chromaticity values}

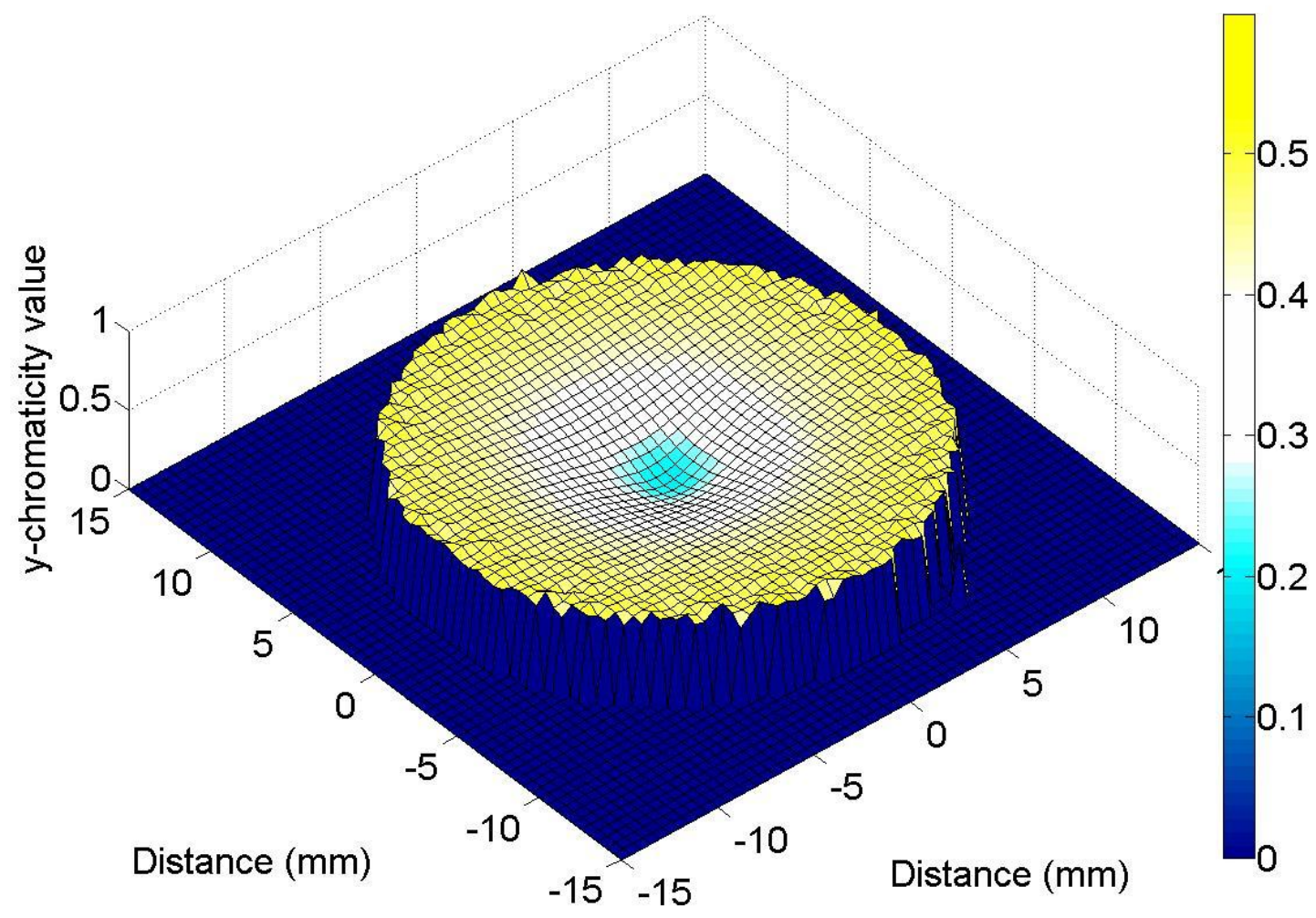

Figure 30 - A graph displaying the color seen when looking at the $\mathbf{2 . 5} \mathbf{~ m m}$ thick, one inch diameter, and 3.0 wt\% phosphor/PDMS sample illuminated with the blue Thorlabs LED. While this graph displays color it does not relay intensity information to display brightness.

Chromaticity Cross Section

Examining a cross-section of the chromaticity values versus radial distance provides data regarding uniformity. The following section examines thicknesses, concentrations, and heights effect on uniformity of color emitted from the phosphor/PDMS substrate. 
Thickness

The thickness of the phosphor/PDMS substrate has a substantial effect on uniformity. Both $\mathrm{x}$ and y chromaticity values are influenced by thickness (Figures 31 and 32). As the thickness increases, the color of light becomes more uniform over the entire substrate surface. The least uniform sample is the thinnest of the tested samples $(0.97 \mathrm{~mm})$. With the concentration of $3.8 \mathrm{wt} \%$ used for this study, $0.97 \mathrm{~mm}$ was not thick enough to have sufficient photoluminescence at the edges of the substrate. Therefore, light was concentrated directly above the LED instead of being diffused over the entire substrate. 1.79 to $4.74 \mathrm{~mm}$ thick substrates were all capable of exhibiting sufficient phosphor excitation over the entire surface.

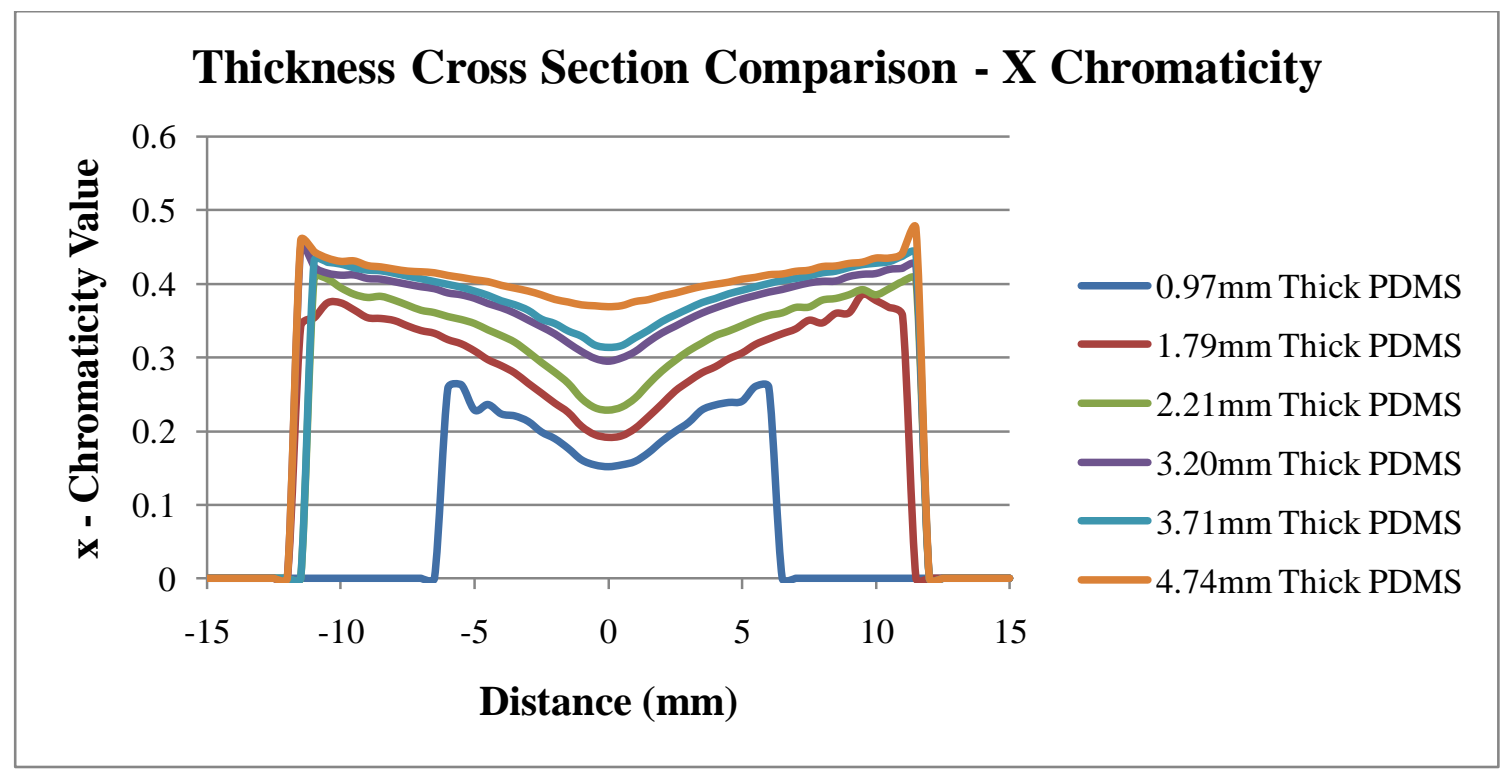

Figure 31 - A cross section of $\mathbf{x}$ - chromaticity values indicating the uniformity of color. A minimal change in chromaticity value indicates a more constant color over the cross section of the substrate. Each thickness experiment was conducted using $3.8 \mathrm{wt} \%$ phosphor in PDMS at a height of $0 \mathrm{~mm}$. 


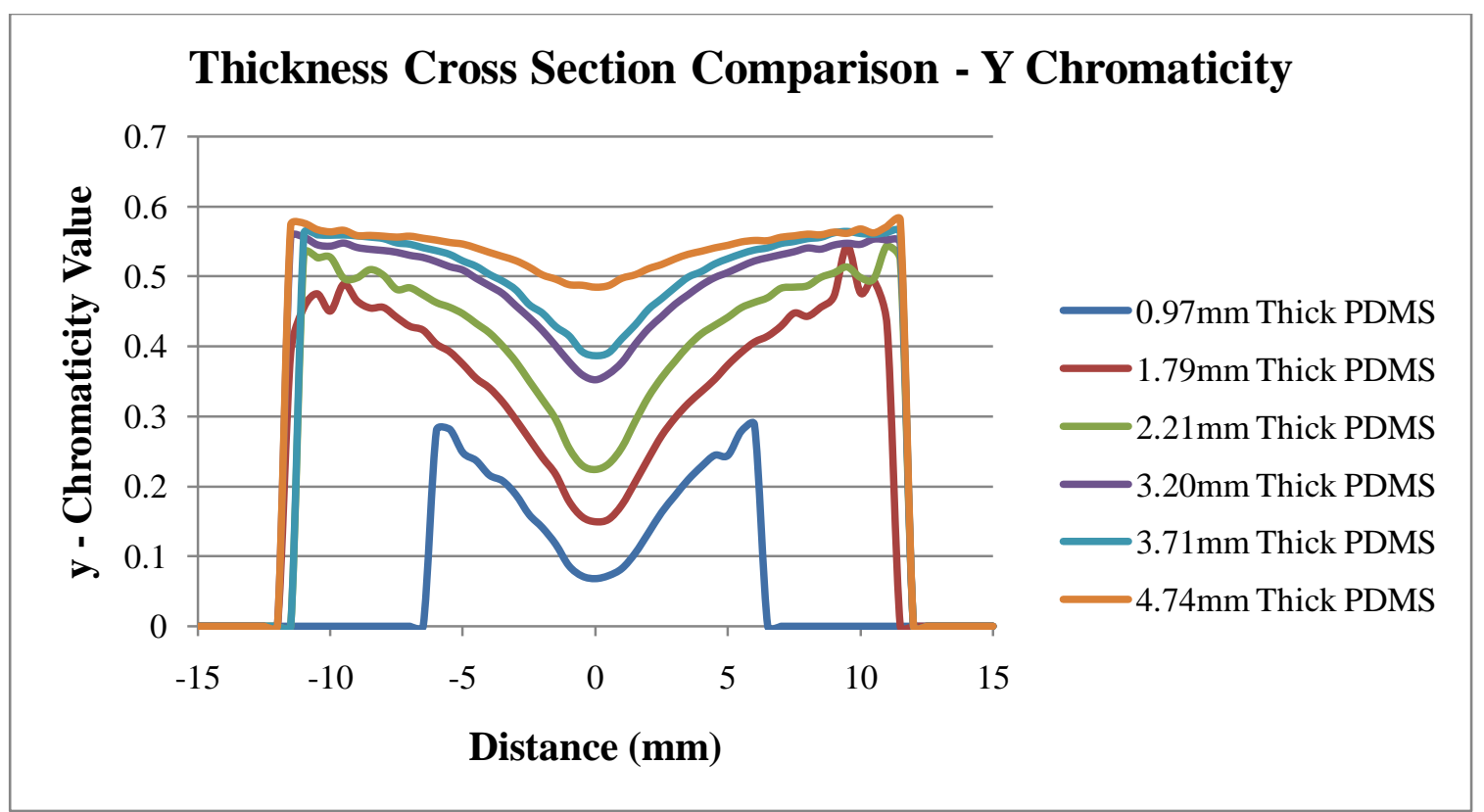

Figure 32 - This graph displays the $y$ - chromaticity values with the same data used to produce Figure 31. Y - chromaticity values are more influenced by changes in phosphor concentration and thickness than $x$ - chromaticity values. Each thickness experiment was conducted using 3.8 wt\% phosphor in PDMS at a height of 0 mm.

Concentration

Test results for concentration displayed similar trends found with the thickness tests (Appendix I and J). The $1.8 \mathrm{wt} \%$ sample had too little phosphor to photoluminescence in the outer radii of the substrate. $2.2 \mathrm{wt} \%$ samples displayed the greatest variance in color while the $3.4 \mathrm{wt} \%$ displayed the least variance. Color variance would decrease with increasing concentration.

Height

As stated earlier, height did not contribute greatly to color values. The region directly above the LED displays nearly constant color despite changes in distance from the LED die (Appendix $\mathrm{K}$ and $\mathrm{L}$ ). The outer region of the substrate displays a slight increase toward a yellow color as the distance is decreased. 
While these plots do indicate how uniform the light production is at various radii across the surface, the uniform samples have an excess of photoluminescence required for creating white light. In this instance, the fact that some surfaces are more uniform than others simply implies that light produced by photoluminescence is dominating the spectrum.

Prediction Matrices and Corresponding Equations

As seen in the chromaticity cross-section plots, there are different trends depending upon $\mathrm{x}$ and $\mathrm{y}$ chromaticity values as well as thickness, concentration, or height variables. In addition to those trends, there is a slightly different behavior of the phosphors ability to adjust chromaticity value as we examine points along the substrate that are not directly above the LED.

In the region directly above the LED, radii of 0 to $1 \mathrm{~mm}$, thickness and concentration variables exhibit a linear chromaticity trends (Figure 33). 


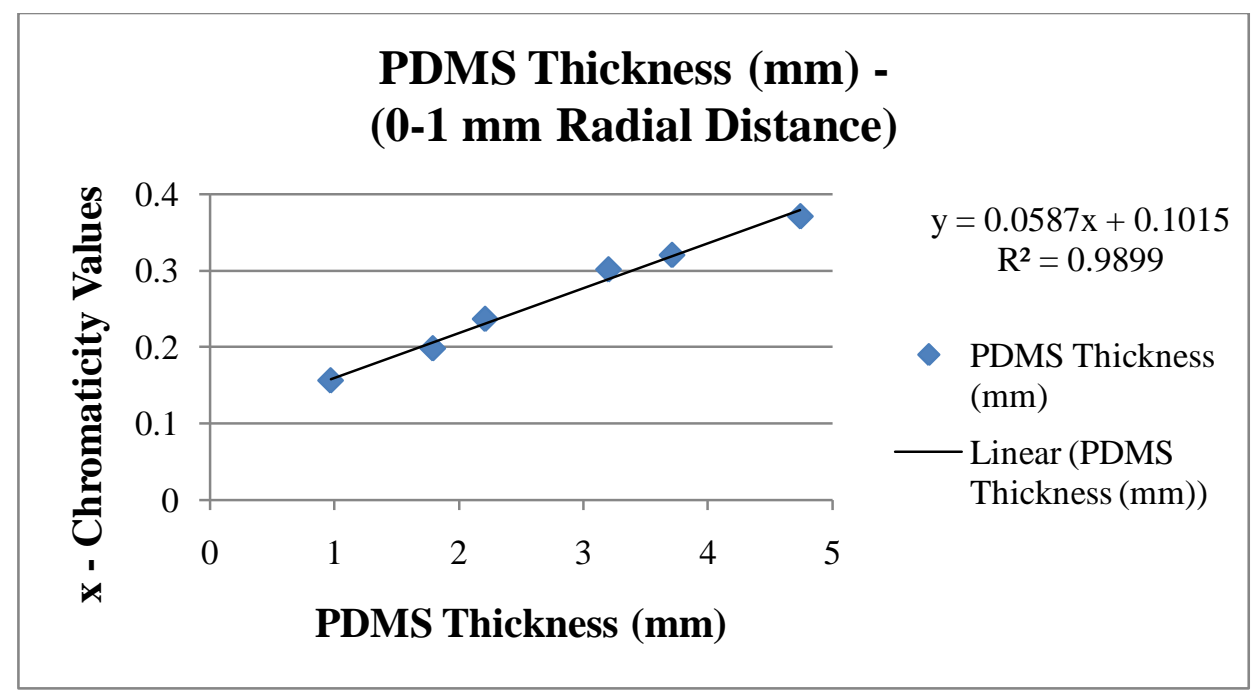

Figure 33 - Using chromaticity values obtained in a small region directly above the LED die during thickness testing of $3.8 \mathrm{wt} \%$ samples, a linear equation was fit to the points to display a slope of 0.0587 ( $x$-chromaticity value/mm).

Offset radial distances (3-6 mm away from the LED dies' center) display a non-linear behavior in chromaticity values (Figuue 33).

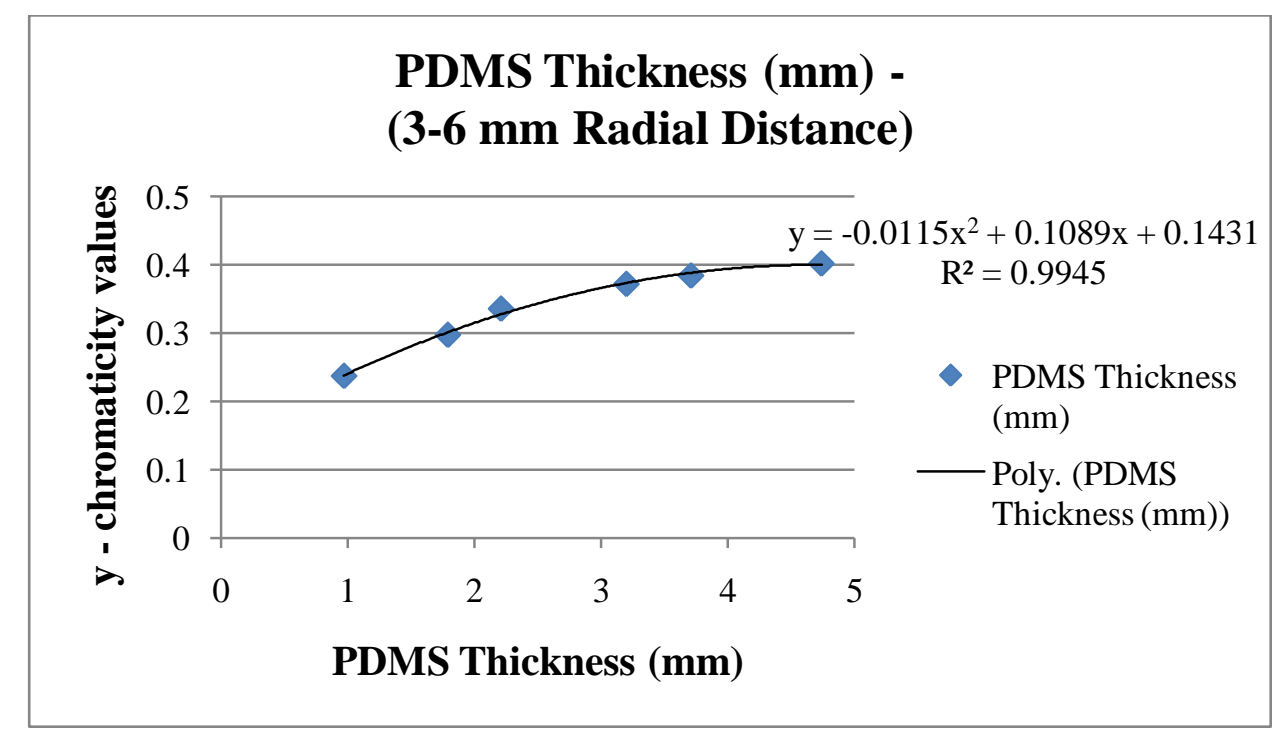

Figure 34 - Using chromaticity values obtained from radii not directly above the LED die during thickness testing of $3.8 \mathrm{wt} \%$ samples, a parabolic equation was fit to the $\mathrm{y}$ - chromaticity points. 
Concentration continues to exhibit a linear trend on the outer regions of the substrate but thickness displays a parabolic trend. Height displays parabolic trends regardless of radii location but, as stated earlier, it has minimal effect on chromaticity values.

It is important to note the difference in behavior which the phosphor exhibits at different radii. For design it will be critical to know the disbursement of LED die below the phosphor to predict the color output in regions with minimal illumination from LEDs.

For each variable an equation is developed for a region from 0 to $1 \mathrm{~mm}$ radial distance above the LED (also referred to as 'Directly Above LED'). An equation is also developed for the radial distance of 3 to $6 \mathrm{~mm}$ above the LED (also referred to as 'Offset Above LED'). Table VII displays the twelve equations that were developed to describe each variables influence on color output. 
Table VIII - Chromaticity Trends

\begin{tabular}{|c|c|c|}
\hline Variable & Location & Equation \\
\hline \multirow{4}{*}{ Thickness } & $(0-1 \mathrm{~mm})$ & $\mathrm{x}$ chroma $=0.0587$ (thickness) +0.1015 \\
\hline & Radii & $\mathrm{y}$ chroma $=0.1127$ (thickness $)+0.0235$ \\
\hline & $(3-6 \mathrm{~mm})$ & $\begin{array}{l}\mathrm{x} \text { chroma }=-0.0115(\text { thickness })^{\wedge} 2+0.1089 \text { (thickness) } \\
+0.1431\end{array}$ \\
\hline & Radii & $\begin{array}{l}\text { y chroma }=-0.0236(\text { thickness })^{\wedge} 2+0.2134 \text { (thickness) } \\
+0.0553\end{array}$ \\
\hline \multirow{4}{*}{ Concentration } & $(0-1 \mathrm{~mm})$ & $\mathrm{x}$ chroma $=0.0488($ concentration $)+0.0777$ \\
\hline & Radii & $\mathrm{y}$ chorma $=0.0985$ (concentration $)+0.081$ \\
\hline & $(3-6 \mathrm{~mm})$ & $\mathrm{x}$ chroma $=0.0516($ concentration $)+0.1529$ \\
\hline & Radii & $\mathrm{y}$ chorma $=0.0993($ concentration $)+0.0781$ \\
\hline \multirow{4}{*}{ Height } & $(0-1 \quad \mathrm{~mm})$ & $\mathrm{x}$ chroma $=0.0002(\text { height })^{\wedge} 2+0.0029$ (height $)+0.2111$ \\
\hline & Radii & $\mathrm{y}$ chroma $=0.0002(\text { height })^{\wedge} 2+0.0045$ (height $)+0.1874$ \\
\hline & $(3-6 \mathrm{~mm})$ & $\mathrm{x}$ chroma $=0.0008(\text { height })^{\wedge} 2-0.0093$ (height $)+0.2907$ \\
\hline & Radii & $\mathrm{y}$ chroma $=0.0008(\text { height })^{\wedge} 2-0.0135$ (height $)+0.3379$ \\
\hline
\end{tabular}

For each region it is also necessary to construct an equation for an $\mathrm{x}$ and a y chromaticity value. Each equation is combined with another to produce a three dimensional graph involving a comparison of two equations against a vertical axis of a given chromaticity value (Figure 35). 


\section{Thickness versus Concentration Offset Above LED (3-6 mm Radial Distance) - x-chromaticity}

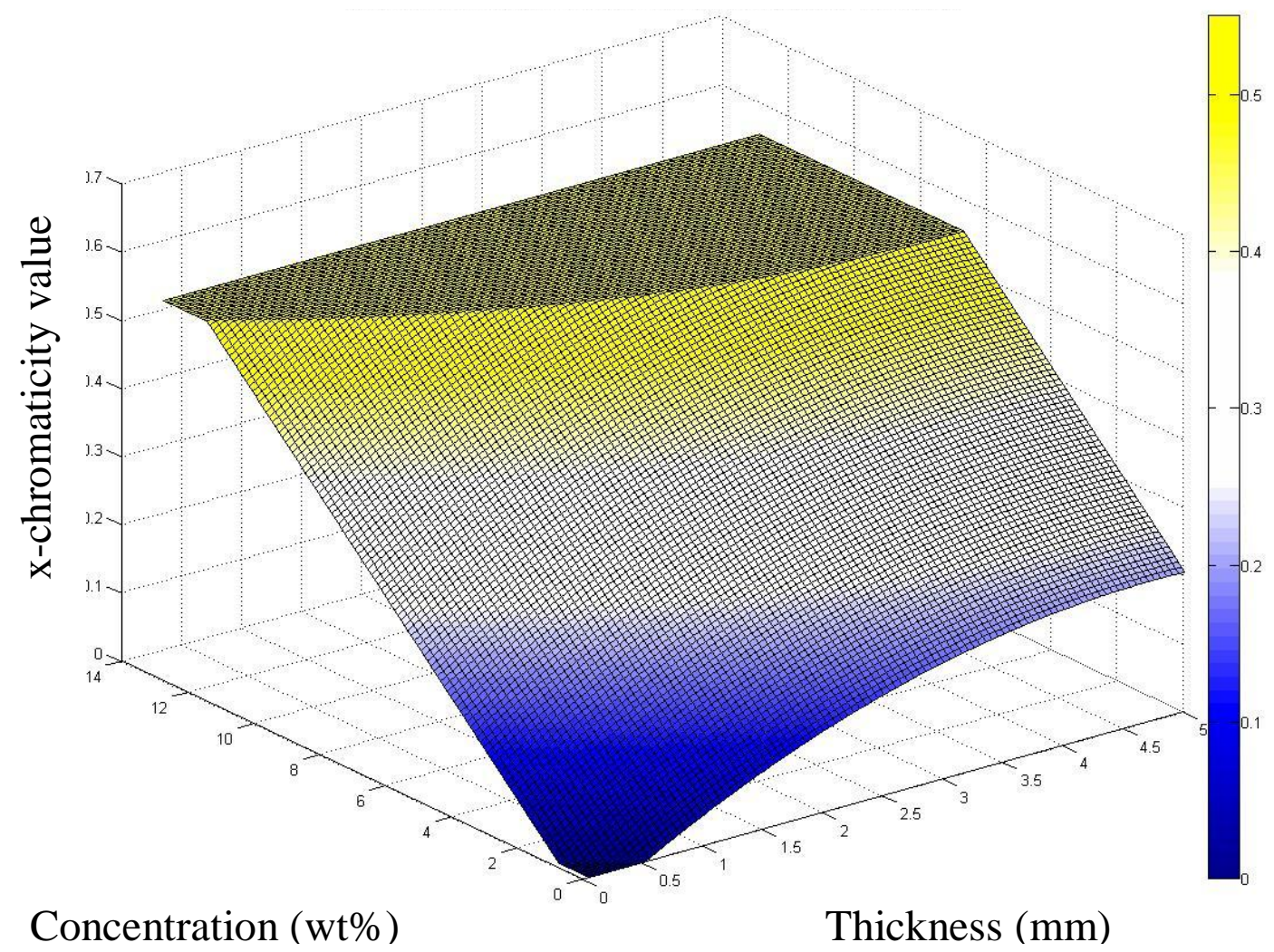

Figure 35 - A 3 dimensional plot color coded to best relay information regarding the actual color of the observed chromaticity values. The plateaus at the bottom and top of the plot are regions which are not valid chromaticity values and therefore are points where the equations fail. This specific plot compares $x$ - chromaticity effects of thickness and concentration over a 3 to $6 \mathrm{~mm}$ radial distance from the LED.

Regions at the top and bottom form a plateau because the equation would normally take that value out of the range of the chromaticity values. Therefore, the plateau is a region where the equation fails and the result of combining the two given variables would yield the most extreme blue or yellow possible with this set up. These three dimensional plots were also produced to compare thickness and concentration at the different radii as well as both chromaticity values (Appendix $\mathrm{M}, \mathrm{N}$, and $\mathrm{O}$ ). Three dimensional plots were 
created to compare height and thickness, also for different radii and $\mathrm{x}$ and y chromaticity values (Appendix P, Q, R, and S). Finally, three dimensional plots were created for height and concentration comparison (Appendix T, U, V, and W).

\section{Conclusions}

The following describes adjustments with regards to phosphor thickness, concentration, and height when designing a multi-Kelvin LED as illustrated in the following chapter.

Phosphor

One concern is the degree for which color will be able to be influenced with the specific phosphor being used. Color values in all of the testing completed with the Thorlabs blue LED and YAG:Ce phosphor have not been able to produce the color values which follow the Kelvin scale of an ideal black body radiator. To address this issue an additional phosphor which emits light somewhere between 585 and $770 \mathrm{~nm}$ will be necessary to influence the chromaticity values enough to mimic the Kelvin scale. Selenium doped zinc sulfide was used because of its ability to photoluminesce in the presence of UV and/or blue light (a constraint applied to this study) while emitting light at $647 \mathrm{~nm}$ wavelengths. The results of $\mathrm{ZnS}$ :Se's influence on the chromaticity values are discussed in the following chapter.

\section{Concentration and Thickness}

The results of this study indicate that higher concentration phosphor/PDMS samples with a thinner thickness are beneficial to efficiency. For the majority of this study a thinner phosphor lens is unnecessary, in addition to being more difficult to process. While efficiency does increase with thinner samples and higher concentrations, the ability to 
diffuse light is limited. An investigation into the ability to compensate for the lack of diffused light will be discussed in chapter VIII.

\section{Height}

It has been made clear by the results of this study that increasing the height greatly decreases intensity. Adjusting the height also has a negligible impact on color output. With those two trends in mind, height will have to be optimized based on temperature readings. To justify moving the phosphor layer away from the LED, there will have to be a substantial decrease in temperature of the substrate with a couple millimeter distance adjustment. For the remainder of testing, height is held in contact directly over the epoxy covered Thorlabs LED. 


\section{CHAPTER VI: CREATING A MULTI-KELVIN WHITE SOURCE USING A BLUE AND UV LED IN CONJUNCTION WITH YAG:Ce AND ZnS:Se PHOSPHORS}

\section{Introduction}

The following chapter examines the feasibility of developing a white light source capable of producing colors between 2500 Kelvin and 7500 Kelvin by simply adjusting amperage to a blue and ultraviolet (UV) light emitting diode (LED). The purpose of a lighting source of this nature is to better replicate daylight inside a building given a time of day. This study investigates that type of white light source using a $385 \mathrm{~nm}$ UV LED, a $457 \mathrm{~nm}$ blue LED, a $479 \mathrm{~nm}$ blue LED, a $562 \mathrm{~nm}$ peak cerium doped yttrium aluminum garnet (YAG:Ce) phosphor, and a $647 \mathrm{~nm}$ peak selenium doped zinc sulfide (ZnS:Se) phosphor.

\section{Crystallography and Photoluminescence of $\mathrm{ZnS}: \mathrm{Se}$}

$\mathrm{ZnS}: S e$ was specifically selected due to the necessity of a red light $(\sim 647 \mathrm{~nm})$ source in combination with the other colors of the set up to create the desired white light source based on LEDs and phosphors. The specific composition of this phosphor enables it to convert blue and ultraviolet light to red light which is necessary for the LED configuration utilized. The zinc sulfide crystal lattice absorbs electromagnetic radiation between 380 and $480 \mathrm{~nm}$ most efficiently (Figure 36). 


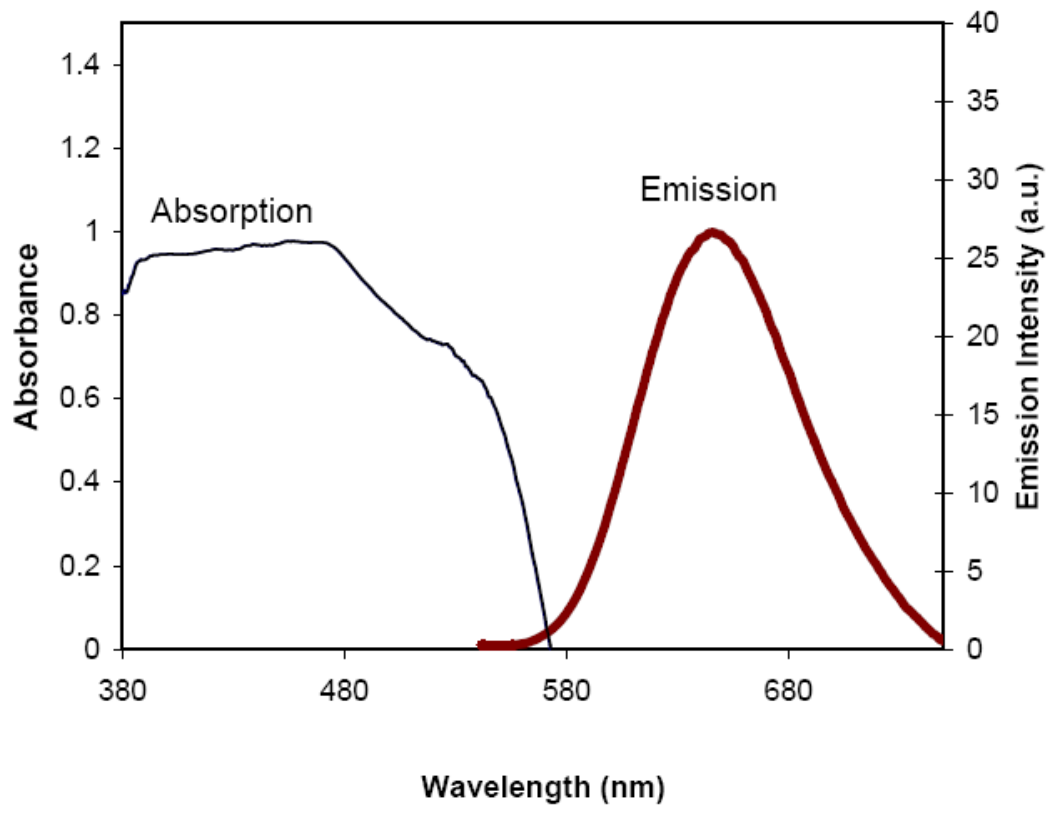

Figure 36 - Absorption and fluorescent behavior of the specific ZnS:Se phosphor used. Absorption shows the ability for $\mathrm{ZnS}$ :Se to absorb a given wavelength and the Emission displays the result of the selenium dopant to produce certain wavelengths ${ }_{(30)}$.

The electron states of the selenium dopant then allow the energy to be re-emitted at the $647 \mathrm{~nm}$ peak-wavelength. This form of luminescence occurs in much less than one second, and is therefore referred to as photoluminescence. These specific phosphor particles create what is referred to as a Wurtzite crystal structure (Figure 5) (31). It consists of alternating layers of zinc forming a hexagonal crystal structure and sulfur forming a hexagonal crystal structure. To bond the homogenous hexagonal sheets, every atom forms a tetrahedron. This results in alternating tetrahedrons, every other one with a zinc atom in the center while the other half has a sulfur atom in its center. In phosphor particles supplied by PhosphorTech, this crystalline structure comprises their approximately $10 \mu \mathrm{m}$ diameter structures (Figure 37). 


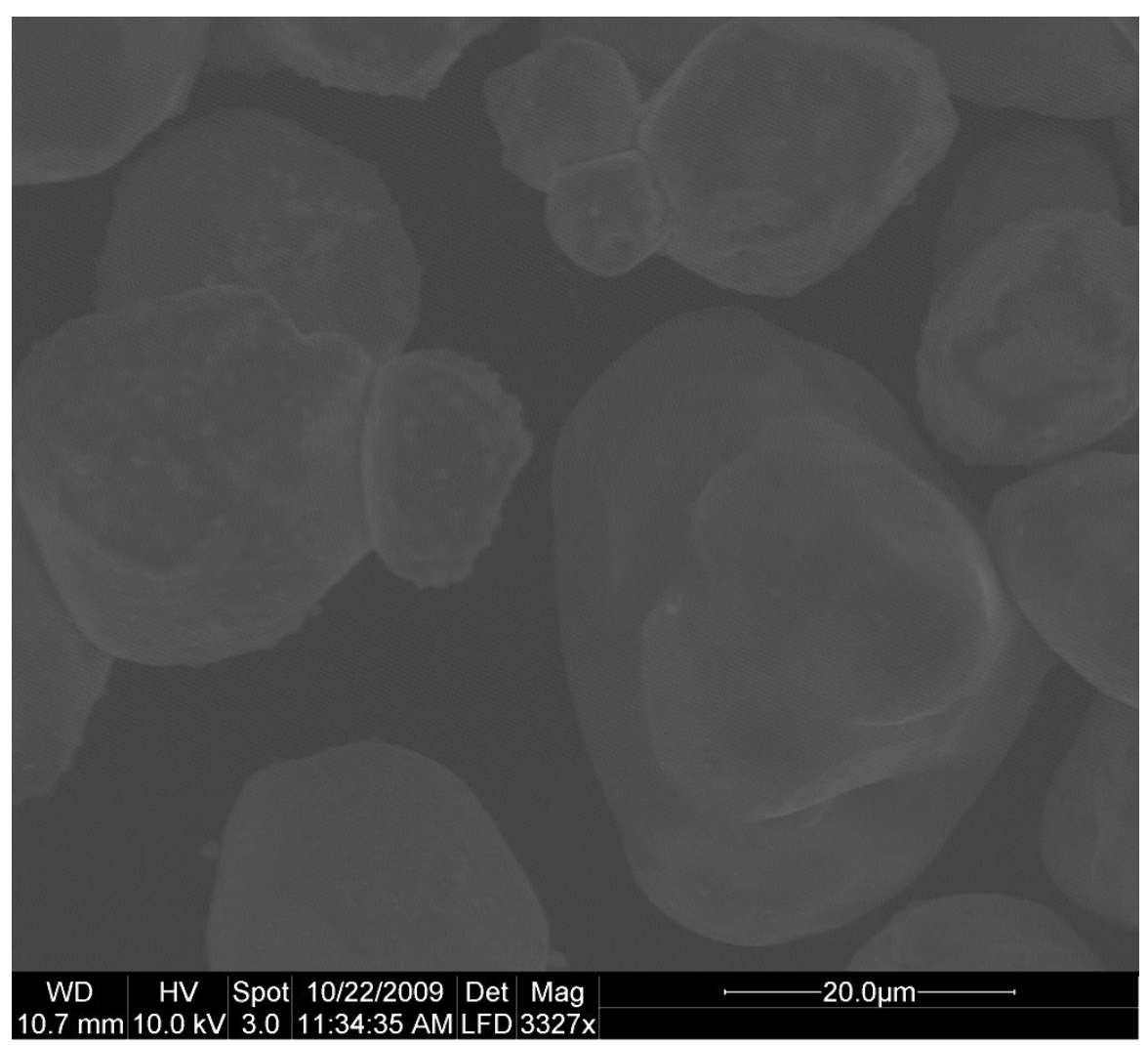

Figure 37 - A SEM image of phosphor particles in low vacuum. Mean particle diameter is stated to be 10 microns by PhosphorTech.

Luminescence Process

Semiconductors play a slightly different role in luminescence than the method discussed for YAG:Ce phosphor. The valence and conduction band are separated with an energy gap of a few eV. Excited electrons enter the empty conduction band and leave a hole in the completely filled valence band. When an electron is able to recombine with a hole the energy gained during that transition may be given off as a specific wavelength of light equal to that energy difference. The majority of the time recombination will occur near or at defects in the crystal lattice. In instances of edge emission (emission which occurs near the same energy level as the absorption) bound excitons are usually the cause. Exciton recombination may happen when an electron or hole is trapped at an 
imperfection in the crystal lattice, forcing the recombination to occur between the same electron and hole that previously went through the excitation. Donor-acceptor pair emission occurs where recombination takes place between an acceptor and a donor. In the case of $\mathrm{ZnS}$ :Se a zinc vacancy becomes the acceptor and a Se ion acts as the donor. Their position in the lattice is essentially constant forcing the distance between these coupled defects to be essentially one value. Strong electron-lattice coupling from the $\mathrm{ZnS}$ :Se host lattice results in broad band emissions (24).

\section{Zinc Sulfide Phosphor Settling During PDMS Cure}

The following section describes the interaction between selenium doped zinc sulfide (ZnS:Se) phosphor particles with polydimethylsiloxane (PDMS). These two materials were mixed together, the same as YAG:Ce was in the previous study, with the intention of creating a stable substrate for photoluminescent particles to reside in. It was found that ZnS:Se settles out of the PDMS completely during the PDMS cure process.

General Properties

The particular problem with ZnS:Se settling while PDMS is curing pertains mainly to the curing mechanism of PDMS. The basic chemical formula is found to be: $\left(\mathrm{H}_{3} \mathrm{C}\right)_{3} \mathrm{SiO}\left[\mathrm{Si}\left(\mathrm{CH}_{3}\right)_{2} \mathrm{O}\right]_{n} \mathrm{Si}\left(\mathrm{CH}_{3}\right)_{3}$. The majority of the chain (monomer) consists of repeating units of the $\mathrm{Si}\left(\mathrm{CH}_{3}\right)_{2} \mathrm{O}$ (Figure 38). 


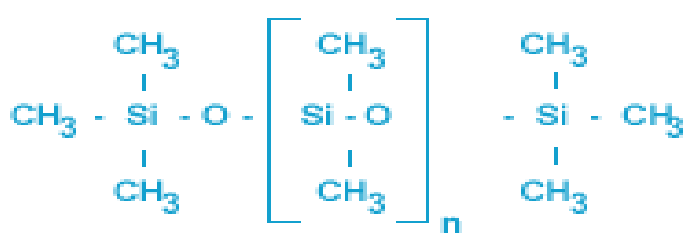

Figure 38 - The basic representation of PDMS backbone. It contains trimethyl groups on the ends of the chain and repeating units of ' $n$ ' amount of the monomer boxed in the center ${ }_{(32}$.

Curing occurs with the introduction of a silane precursor known as methyltrichlorosilane.

The precursor is capable of promoting cross-linking when a catalyst such as platinum is exposed to the polymer.

Dow Corning Specifics

Sylgard 184 by Dow Corning is the specific brand of PDMS used for this study. They sell a two part system and recommend mixing them with the ratio of 10 parts base to 1 part agent. The base contains the vinyl groups (the sections of the polymer chain with carbon double bonds) while the agent contains the catalyst and silane groups (silicon surrounded by four hydrogen atoms) necessary for cross-linking (Figures 39, 40) (33).

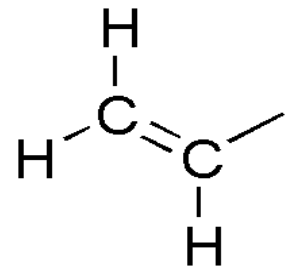

Figure 39 - Vinyl group found in PDMS at sections which have not been cross-linked. The carbon-tocarbon double bond is the key to a vinyl group.

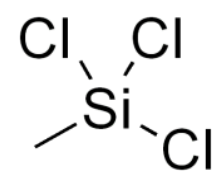

Figure 40 - Silane group found within the agent which is necessary for a bonding site to the PDMS polymer backbone. 
Cure times vary based on temperature. While investigating the settling issue three different cure rates were selected. The longest being 48 hours at room temperature, 1 hour at $60^{\circ} \mathrm{C}$ (the most commonly used in Cal Poly's microfabrication lab), and a short interval of just 10 minutes at $150^{\circ} \mathrm{C}$. All of which produced the same phosphor settling result.

Observations

Regardless of curing temperature and elapsed time, mentioned previously, the $\mathrm{ZnS}: \mathrm{Se}$ particles always settle out. It is referred to as "settling" in this report because of the tendency for the phosphor to condense on the lowest point (direction of gravity) possible. This is opposed to diffusion which would occur in all directions assuming the interfaces are the same. Curing takes place in a plastic Petri dish which contacts the sides and bottom surfaces leaving the top surface exposed to air. Another important observation is that the PDMS still ends up curing and does so with no apparent change in properties. Physically the cured PDMS acts the same as if it were cured without phosphor particles introduced in it and the optical properties appear intact. The final result is that an apparently uniform spread of $\mathrm{ZnS}$ :Se remains in a gel-like substance beneath the cured PDMS (Figure 41).
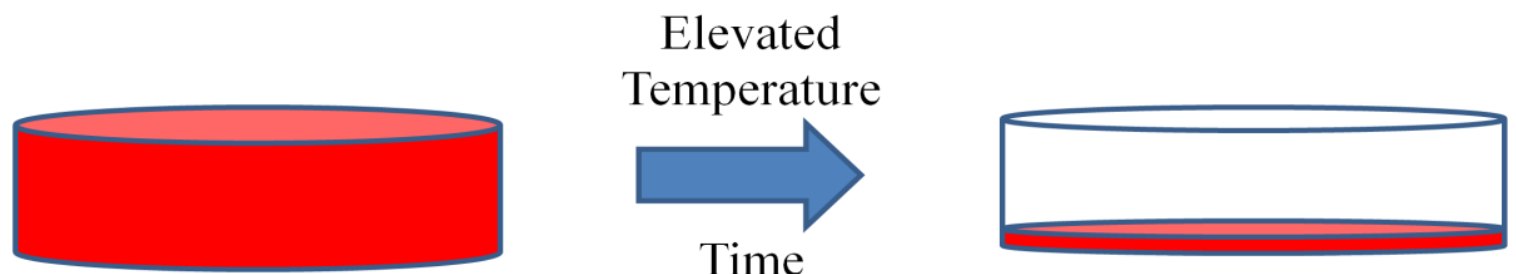

Figure 41 - Left: Simple representation of the $90 \mathrm{~mm}$ diameter by $2.5 \mathrm{~mm}$ height cast of PDMS mixed with the red ZnS:Se phosphor prior to curing. Right: Cured PDMS without any phosphor particles and a concentrated $\mathrm{ZnS}$ :Se layer at the bottom of mold. 


\section{Research}

Dow Corning provides important information useful to this study about their products, which went unnoticed until after settling was observed. A short but fact-filled section of their elastomer information document listed materials which may inhibit curing (34). Organometallics (materials which contain carbon covalently bonded to usually a metallic element), amines (molecules containing a nitrogen with an electron pair), sulfur containing materials, and chlorides (most commonly found in solder fluxes) made up the majority of this list. This implies that many transition metals, post-transition metals, semi-metals, nitrogen, sulfur, chlorine, and even selenium will not be capable of remaining in the polymer while it is curing. All three of the elements making up the $\mathrm{ZnS}: \mathrm{Se}$ phosphor were part of this list. First to note is that the selenium concentration is low and the probability of it being on the surface of the phosphor particle is extremely low. The second note is that zinc and selenium were both listed under "organometallics" and neither is bonded to a carbon atom within these phosphors. The last element to consider is sulfur and in Dow Corning's document it was clearly indicated, any material which contains sulfur will hinder curing.

\section{Cross-Linking}

As stated previously, cross-linking is the mechanism which occurs during the curing of PDMS. To understand why sulfur inhibits the cure it is necessary to understand the cross-linking process. By definition, cross-linking is the joining of two polymer chains via covalent or ionic bonds. The cross-linking mechanism within PDMS is the joining of a hydrosilyl ( $\mathrm{Si}-\mathrm{H})$ and a vinyl group in the presence of a catalyst ${ }_{(35)}$. This process is represented in chemistry as: $-\mathrm{Si}-\mathrm{CH}=\mathrm{CH}_{2}+\mathrm{H}-\mathrm{Si}$ - Catalyst $-\mathrm{Si}-\mathrm{CH}_{2}-\mathrm{CH}_{2}-\mathrm{Si}$-. The most 
common catalyst used for PDMS processing, though not confirmed to be what Dow Corning uses, is what's called cis-dichlorobis(diethylsulfide)platinum(II) (Figure 42) (36). This molecule is represented by: (cis- $\left\{\mathrm{Pt}\left[\left(\mathrm{C}_{2} \mathrm{H}_{5}\right)_{2} \mathrm{~S}_{2} \mathrm{Cl}_{2}\right\}\right)$.

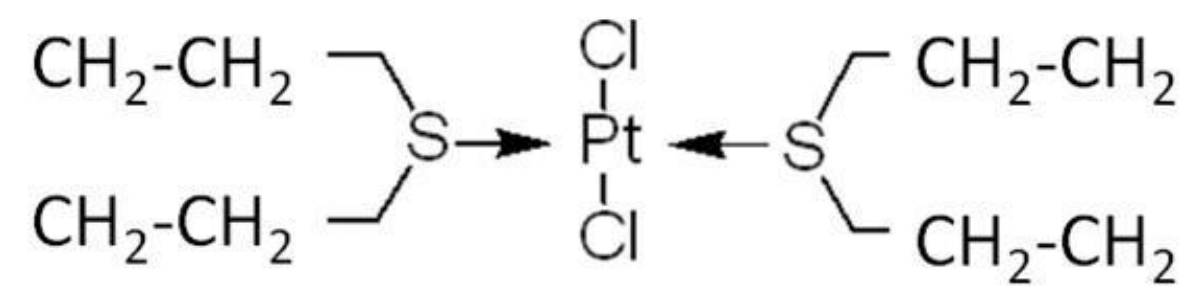

Figure 42 - The catalyst molecule which contains platinum, essential for inducing cross-linking ${ }_{(37)}$. Elements which specifically inhibit the curing with a platinum catalyst are amines, sulfur, chromium, and tin (38). Again, sulfur being one of the "poison" suspects.

\section{Gel Point}

Further research indicated that sulfur is commonly used as a method to examine a polymers gel point when platinum is the catalyst. The gel point is defined as a point at which a specified fraction of monomers appear in the cross-links ${ }_{(39)}$. Essentially it is where 50 percent of the polymer is in a liquid state and the other 50 percent has crosslinked to form a solid substance. Introducing sulfur is a common method of stopping the cross-linking reaction with minimum influence on the polymer properties.

\section{Hydrosilylation}

The process of adding a portion of a silane group to an unsaturated bond (such as a carbon double bond) is referred to as hydrosilylation (Figure 43). It occurs when platinum is reduced (gains an electron) by a silicone hydride molecule ${ }_{(40)}$. In the specific instance of the PDMS cross-linker, hydrosilyl is the element which reduces platinum. The platinum atom then reacts with olefin in the surrounding system. Olefin is a 
compound containing at least one carbon-to-carbon double bond. This is known as the vinyl group in the PDMS polymer chain. Hydrosilylation is also known as addition curing (32).

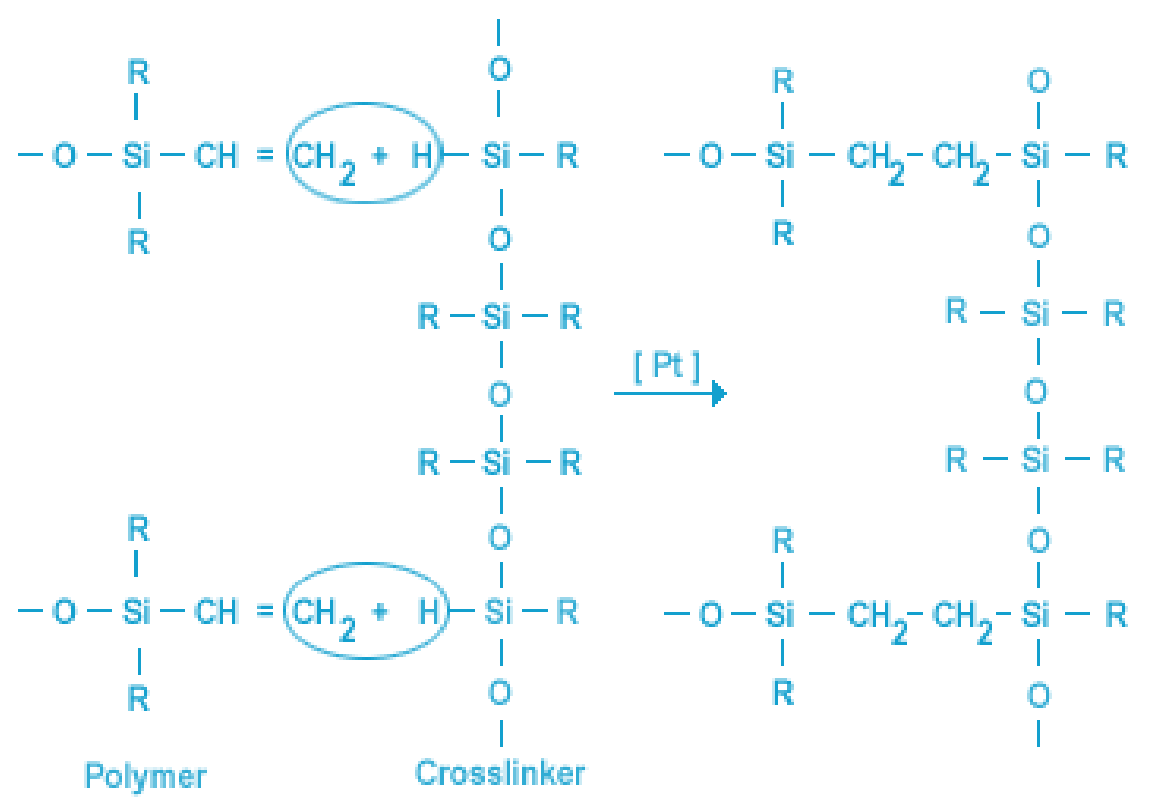

Figure 43 - Left: Part of the polymer backbone which contains the vinyl group. Middle: Crosslinker molecule containing the hydrosilyl group. Right: With the addition of platinum, a cross-linked PDMS forming a solid structure.

Platinum is able to orchestrate this reaction by adsorbing both the silane and vinyl group on it. This process is referred to as chemisorption since covalent bonds are formed with the platinum atom and maintained until the bimolecular reaction occurs. The overall kinetics of the problem can be described with the Langmuir-Hinshelwood equations (41).

\section{Poisonous Sulfur}

Poisonous sulfur unfortunately also chemisorbs onto platinum thereby blocking the active catalyst sites. The damage to the catalyst can be irreversible or reversible. If the bonding strength of surrounding atoms in the system is weaker than sulfurs with the catalyst then 
the sulfur won't break away from platinum. Of course, if the opposite is true then the sulfur to platinum bond will eventually be broken. In low concentrations the LangmuirHinshelwood reaction is prevented ${ }_{(42)}$. In high concentrations platinum is essentially inert to the surrounding polymers.

Hypothesis

Based on the above information it is proposed that surface sulfur atoms on the $\mathrm{ZnS}: \mathrm{Se}$ phosphor particles attract the platinum in the PDMS uncured solution. This may occur because of unsatisfied bonding inherent on surfaces of materials. If the platinum based catalyst attaches itself to phosphor particles it would be unable to adsorb silane and vinyl groups, necessary for cross-linking to occur. The PDMS would hence stay in a viscous form enabling $\mathrm{ZnS}$ :Se to settle out. This corresponds to the observations of different cure rates having no affect on the final result. High temperatures decrease the viscosity of the uncured PDMS and the $\mathrm{ZnS}: \mathrm{Se}$ settles out at a faster rate than low temperatures. When $\mathrm{ZnS}: \mathrm{Se}$ is no longer in the vicinity of remaining platinum and potential cross-linked groups the PDMS will begin to solidify (Figure 44).
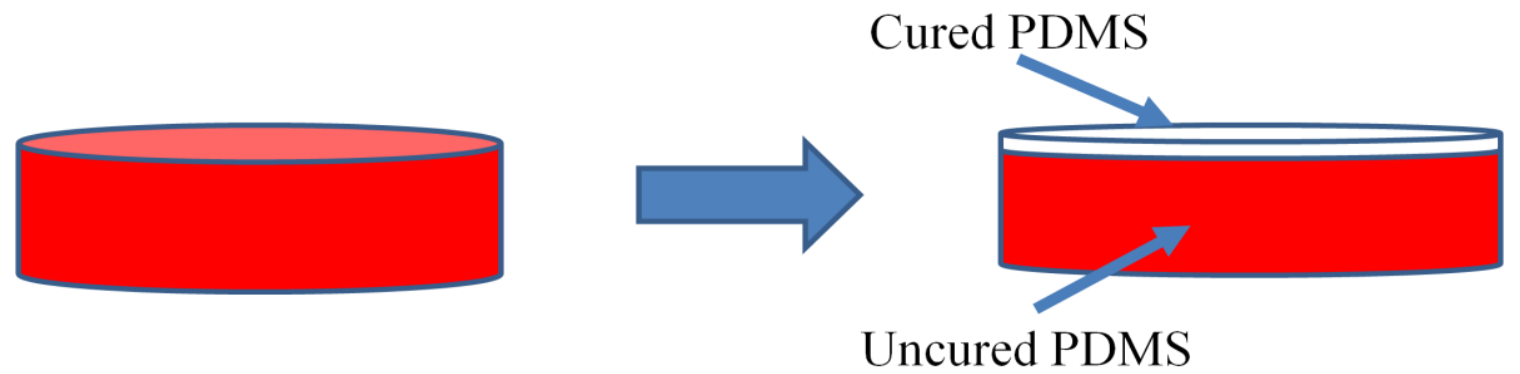

Figure 44 - Representation of what is hypothesized to be occuring during the cure of PDMS and ZnS:Se. Left: A mold containing uniformly mixed phosphor in PDMS. Right: Given time to settle, $\mathrm{ZnS}:$ Se-free-PDMS at the top is allowed to cure. 
Essentially as gravitational forces settle out $\mathrm{ZnS}: \mathrm{Se}$, the top will be capable of solidifying. This theory fits with the observation that all the $\mathrm{ZnS}$ :Se settles on the bottom surface under the cured PDMS and not at the edges or the top surface. The result is that as the $\mathrm{ZnS}: \mathrm{Se}$ settles out of the uncured PDMS the region above is finally able to cure as it normally would (Figure 45).

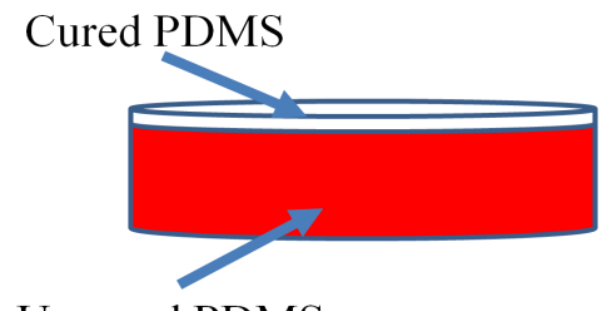

Uncured PDMS

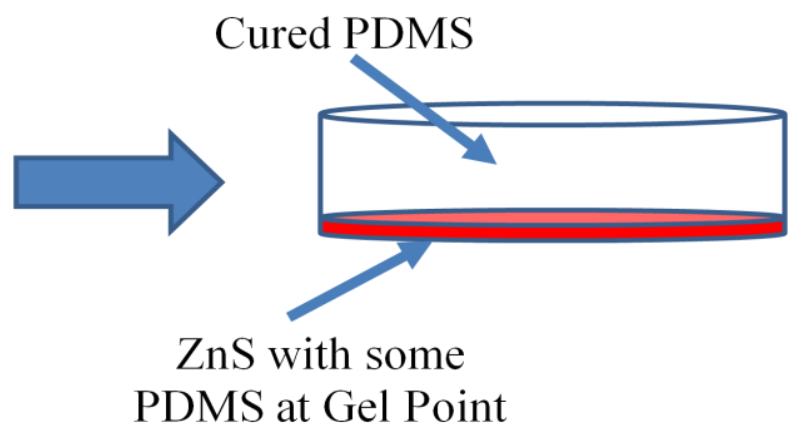

Figure 45 - Left: A duplicate image found on the right hand side of Figure 44. Right: With additional time, all of the ZnS:Se settles out and PDMS is allowed to cure in its absence. What is left behind is a fully cured PDMS sample and ZnS:Se particles at the bottom in a mixture of uncured PDMS.

$\mathrm{ZnS}: \mathrm{Se}$ can be found at the bottom in a gel-like substance which is uncured (not crosslinked) PDMS. This occurs since the concentration of $\mathrm{ZnS}: \mathrm{Se}$ is too high in that region along with the fact that it can no longer settle away from the PDMS.

\section{Silicone Oil Treatment}

One solution to this problem is to prevent the phosphor particles from interacting with the platinum ions during PDMS cure. Silicone oil was used to coat the phosphor particles prior to mixing them into a PDMS solution. Upon weighing a desired amount of $\mathrm{ZnS}: \mathrm{Se}$ phosphor, silicone oil was added with a weight equal to twice that of the $\mathrm{ZnS}: \mathrm{Se}$ particles. A brief study indicated that using three times or four times the amount by weight of silicone oil to $\mathrm{ZnS}$ :Se resulted in more settling during cure with the most 
amount of silicone oil yielding the greatest amount of settling. Silicone oils with the viscosity of 20, 1000, and 30000 centistokes (CS) were used to determine what best prevented $\mathrm{ZnS}$ :Se from settling out. 20 and $30000 \mathrm{CS}$ both resulted in an increase in ZnS:Se settling as compared to the $1000 \mathrm{CS}$. Sylgard 184 PDMS has approximately $4000 \mathrm{CS}$ which may indicate that the optimal silicone oil to encapsulate $\mathrm{ZnS}$ :Se prior to combining with PDMS may be one with the same viscosity (4000 CS). Other tests also indicated that settling is reduced if PDMS is added to an already mixed $\mathrm{ZnS}: \mathrm{Se}$ and silicone oil solution, degassed, and then poured into the mold. Adding $\mathrm{ZnS}$ :Se and silicone oil to PDMS or degassing the mixture after it is in the mold will result in more settling. The process may be further improved with results from a DOE that investigate the optimal viscosity of silicone oil, the weight ratio of silicone oil to $\mathrm{ZnS}: \mathrm{Se}$, and alternative methods to introduce PDMS into the $\mathrm{ZnS}: \mathrm{Se}$ and silicone oil mixture. This treatment enables ZnS:Se and YAG:Ce to exist within a PDMS substrate allowing studies to continue with all three materials.

\section{CIE Chromaticity Behavior Observed}

An important behavioral trait with the 1931 CIE Chromaticity Diagram is that any adjustment made between two light sources is linear. Therefore if a system exists with two light sources any color value which lies directly between their chromaticity coordinates can be obtained (Figure 46). 


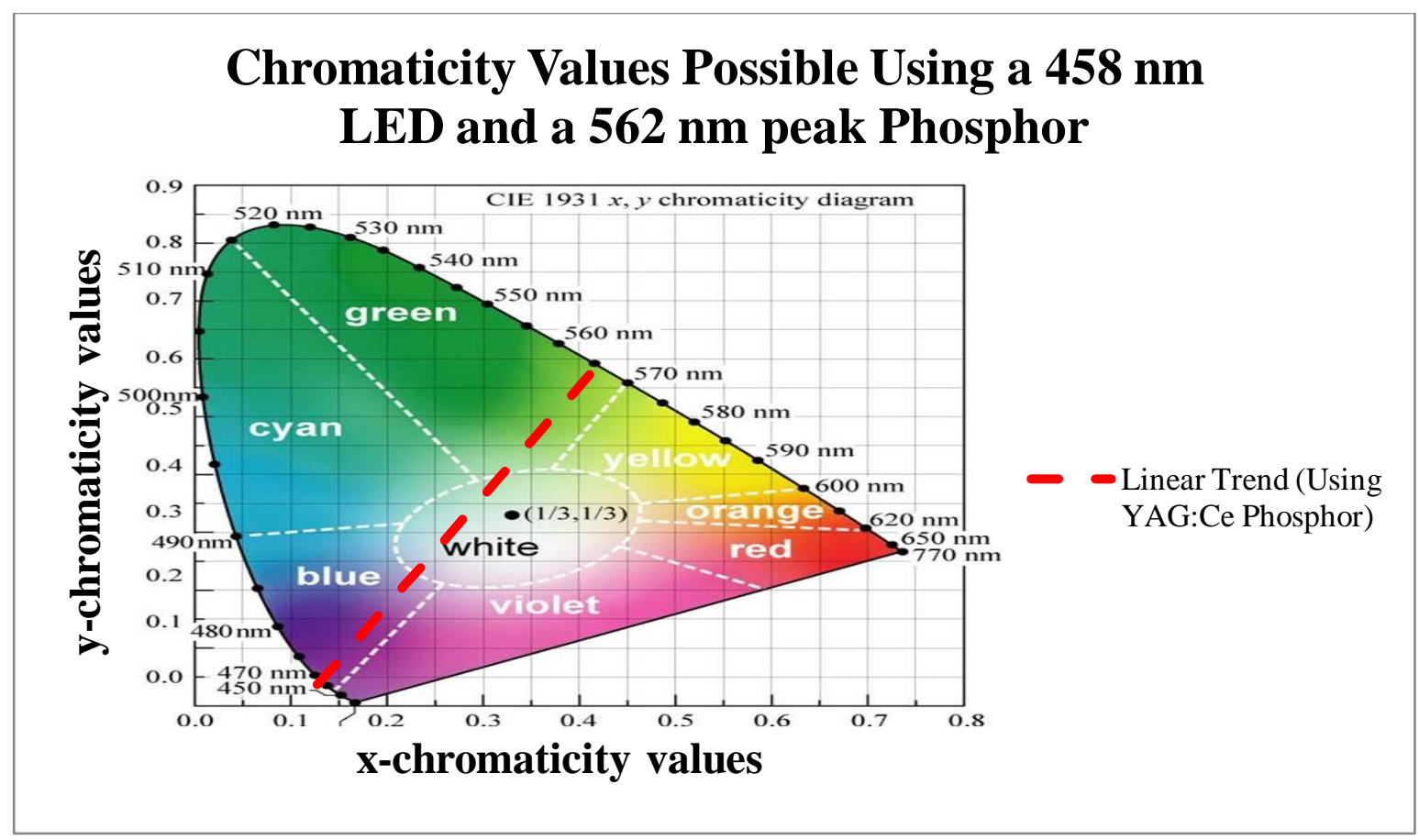

Figure 46 - Any color between the chromaticity value of the yellow phosphor and the chromaticity value of the blue LED can be obtained by changing intensities of one or the other. This was completed in a previous study by adjusting the concentration of the yellow phosphor which inherently changed its intensity while the blue LED was held at maximum current.

All that is necessary is that their intensity be adjusted independently. A single set up with a blue LED and a yellow phosphor (YAG:Ce) cannot adjust its color point with changes in amperage because the phosphors intensity is directly proportional to the blue LEDs intensity.

To produce colors in an area (not a line) there must be at least three different color sources. Adding a red phosphor ( $\mathrm{ZnS}: \mathrm{Se})$ to the combination of a blue LED and a yellow phosphor enables a large majority of white color values to be obtained (Figure 47). 


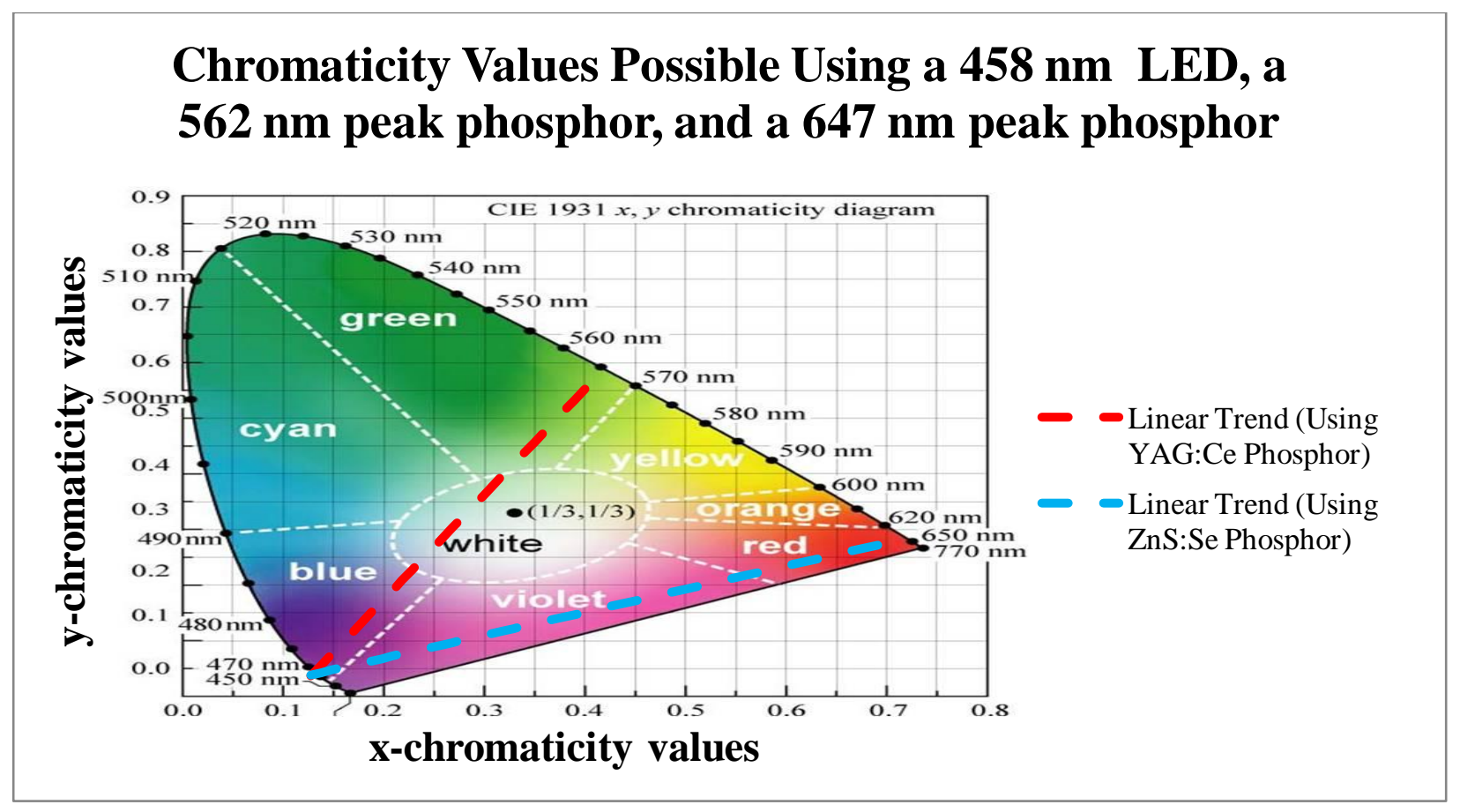

Figure 47 - In between the dashed red and blue lines are all the color values possible using YAG:Ce, ZnS:Se, and a $457 \mathrm{~nm}$ blue LED.

However to obtain a different color value with one set up the intensities of the yellow, red, or blue light sources must change independently of the others. It was postulated that the red phosphor could be adjusted independently with an UV light. The UV light does not excite the yellow phosphor and has little contribution to the color by itself since its peak wavelength is invisible to human eyes. The UV light proved to be capable of making color adjustments by providing extra stimuli to the red phosphor. However, the linear change of the original color value to a more red color value does not follow the Kelvin scale. 


\section{ZnS:Se and YAG:Ce Testing Results}

Various phosphor concentrations, layer orientations, and relative UV and blue LED amperages concluded that the Kelvin scale would not be mimicked with the materials at hand. Prior to addressing the settling issue, $\mathrm{ZnS}$ :Se was held in place between two pieces of PDMS. One piece being clear and the other containing $3.8 \mathrm{wt} \%$ YAG:Ce. Tests were initially conducted with the $\mathrm{ZnS}: \mathrm{Se}$ phosphor on the side closest to the light source to enable as much UV light to be abosrbed as the set up would allow. In that configuration the UV and blue LEDs were adjusted between 0 and 100\% intensity (while holding the other constant). With only the UV light illuminating the two phosphors a chromaticity value of $\mathrm{x}=0.605$ and $\mathrm{y}=0.341$ (red light) was obtained. As blue light was applied to the already luminescing red phosphor it further intensified the light while simultaneously exciting YAG:Ce. The result was a linear change in chromaticity values to $x=0.401$ and $y=0.403$ (white light). Decreasing the UV LED intensity to zero revealed the minimal effect UV light had on the ZnS:Se phosphor relative to the blue light. Chromaticity values shifted by a small amount to $\mathrm{x}=0.388$ and $\mathrm{y}=0.407$ (white light). The test was repeated placing the YAG:Ce phosphor toward the light source and the ZnS:Se phosphor away. The results behaved in a similar manner but with a deviation in chromaticity values due to a decrease in measured YAG:Ce emission caused by diffusion at the ZnS:Se surface (Figure 48). 


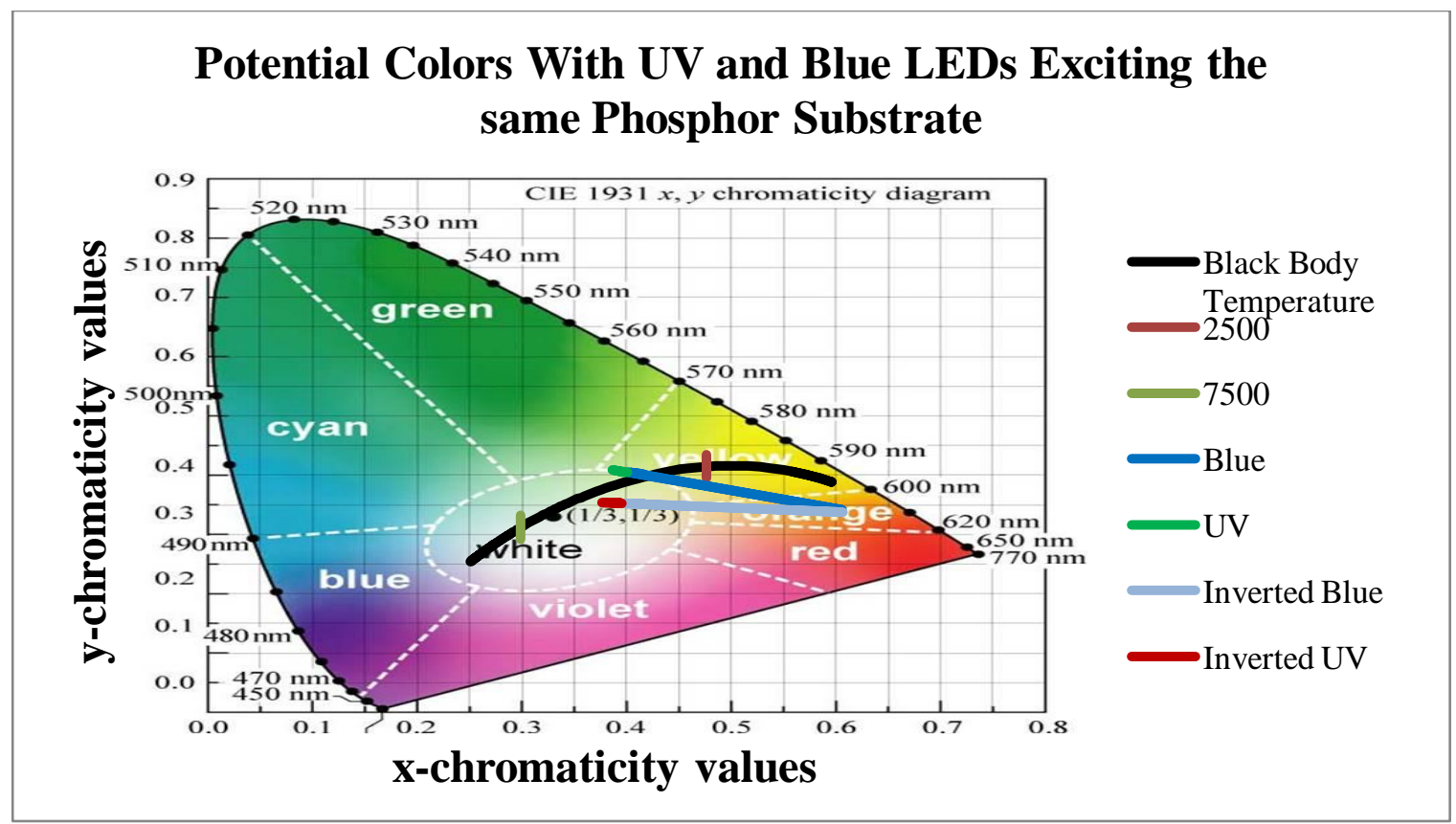

Figure 48 - One experiment indicating ZnS:Se and YAG:Ce behavior when simultaneously exposed to blue and UV LEDs. 'Inverted' indicates the color values observed when the thin layer of ZnS:Se was toward the integrating sphere as opposed to towards the light source. The lines represent all the color values possible by changing amperage to their corresponding LED.

Three major behaviors were noted following this test. The first is that UV light had negligible effect on the overall color of the system when in combination with blue light. The second observation compares the slope of the Kelvin line to the chromaticity values of the set up. Essentially the two light sources of the system are red light from UV stimulated $\mathrm{ZnS}: \mathrm{Se}$ and white light from blue stimulated YAG:Ce + ZnS:Se. As noticed before, only chromaticity values in between those two colored light sources are obtainable. These two observations support the third behavior where any color on the Kelvin scale may be obtained by the two phosphors and two LEDs currently used but only if concentrations were to change. 


\section{CHAPTER VII: METHOD TO APPROXMIATE KELVIN VALUES BETWEEN 2500 AND 7500 IN A LINEAR MANNER}

\section{Introduction}

The most feasible way of mimicking the Kelvin scale is to approximate its values in a linear manner since adjusting single intensities of light sources behaves linearly. The closest linear approximation to the Kelvin scale between 2500 and 7500 Kelvin turned out to be a line ranging from $484 \mathrm{~nm}$ to $583 \mathrm{~nm}$ (Figure 49).

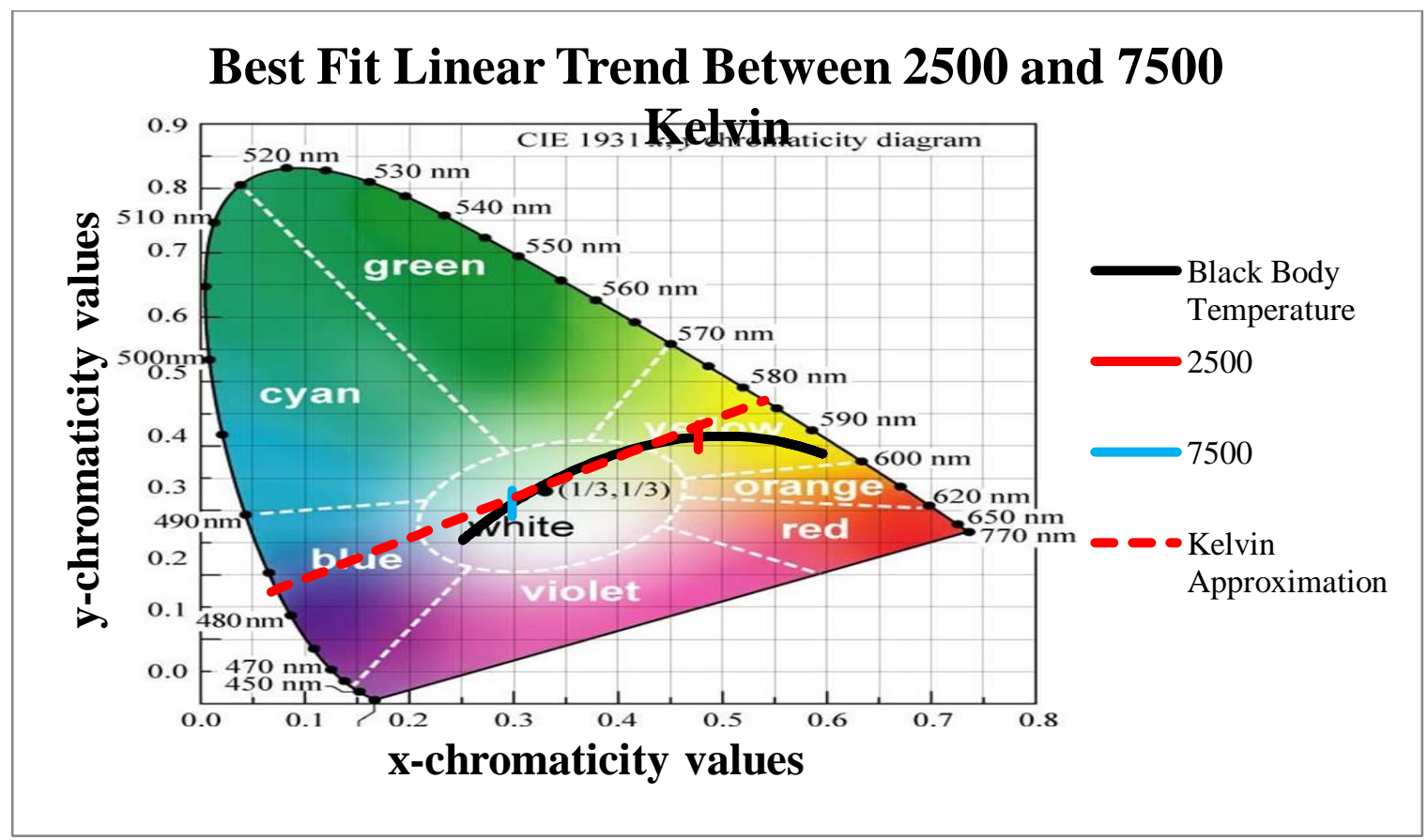

Figure 49 - The simplest method to approximate the Kelvin temperatures between 2500 and 7500 is to adjust the intensities of color sources with chromaticity values along the linear approximation curve outside of the 2500 to 7500 interval.

Hence, the best method to model that linear range would be to adjust the intensities of a $484 \mathrm{~nm}$ (blue) light source and a $583 \mathrm{~nm}$ (yellow) light source independently of each other. 


\section{Color Rendering Index}

There are other factors to consider when designing this light source. The Color Rendering Index (CRI), essentially a measurement of the light sources ability to illuminate a variety of different colored surfaces, would be minimal if the apparatus consisted of just a blue LED and a yellow LED. To improve the color rendering index the spectrum must be broadened. Phosphor is an excellent material to increase the CRI since it re-emits light in a broader spectrum than the LED produces. Combining a red and yellow phosphor not only further broadens the spectrum but substantially increases the white light source's ability to illuminate red and violet objects. Therefore, it is not necessarily best to approximate the curve using a $583 \mathrm{~nm}$ yellow LED since a blue LED and two phosphors would improve the CRI.

\section{Variable White Light with Two LEDs (Blue and UV) and a Phosphor Substrate}

As mentioned previously, a $457 \mathrm{~nm}$ LED, $562 \mathrm{~nm}$ peak phosphor (YAG:Ce), and $647 \mathrm{~nm}$ peak phosphor ( $\mathrm{ZnS}: \mathrm{Se})$ were selected to produce the various color values along the Kelvin scale while maintaining a high CRI. However the intensity of the blue light source must be adjusted independently of the phosphors to follow the Kelvin scale. Since both phosphors depend on the blue light a second blue light source is necessary to address this problem.

To maintain the restriction of using a single substrate of phosphors above a blue and UV LED, the most reasonable answer to this problem is to obtain a blue phosphor which is excited exclusively by the UV LED. Two options are available in this instance. We 
could combine two phosphors, one in the blue and the other in the green region, to obtain the specific color value desired or we could use one blue phosphor with a peak close to our desired color. BAM:Eu $\left(\mathrm{BaMgAl}_{10} \mathrm{O}_{17}: \mathrm{Eu}^{2+}\right)$, with a peak wavelength of $488 \mathrm{~nm}$, was selected to solve this issue and minimize the variety of phosphors used in the set up.

Acknowledging the constraint that the blue phosphor emits at $488 \mathrm{~nm}$, a linear approximation of the Kelvin scale was calculated with the least possible deviation (Figure 50). The result indicates all the chromaticity values desired between the 2500 and 7500 Kelvin values on the chromaticity diagram. The desired chromaticity coordinate closest to 2500 and 7500 Kelvin turned out to be $(0.476,0.398)$ and $(0.301,0.336)$ respectfully.

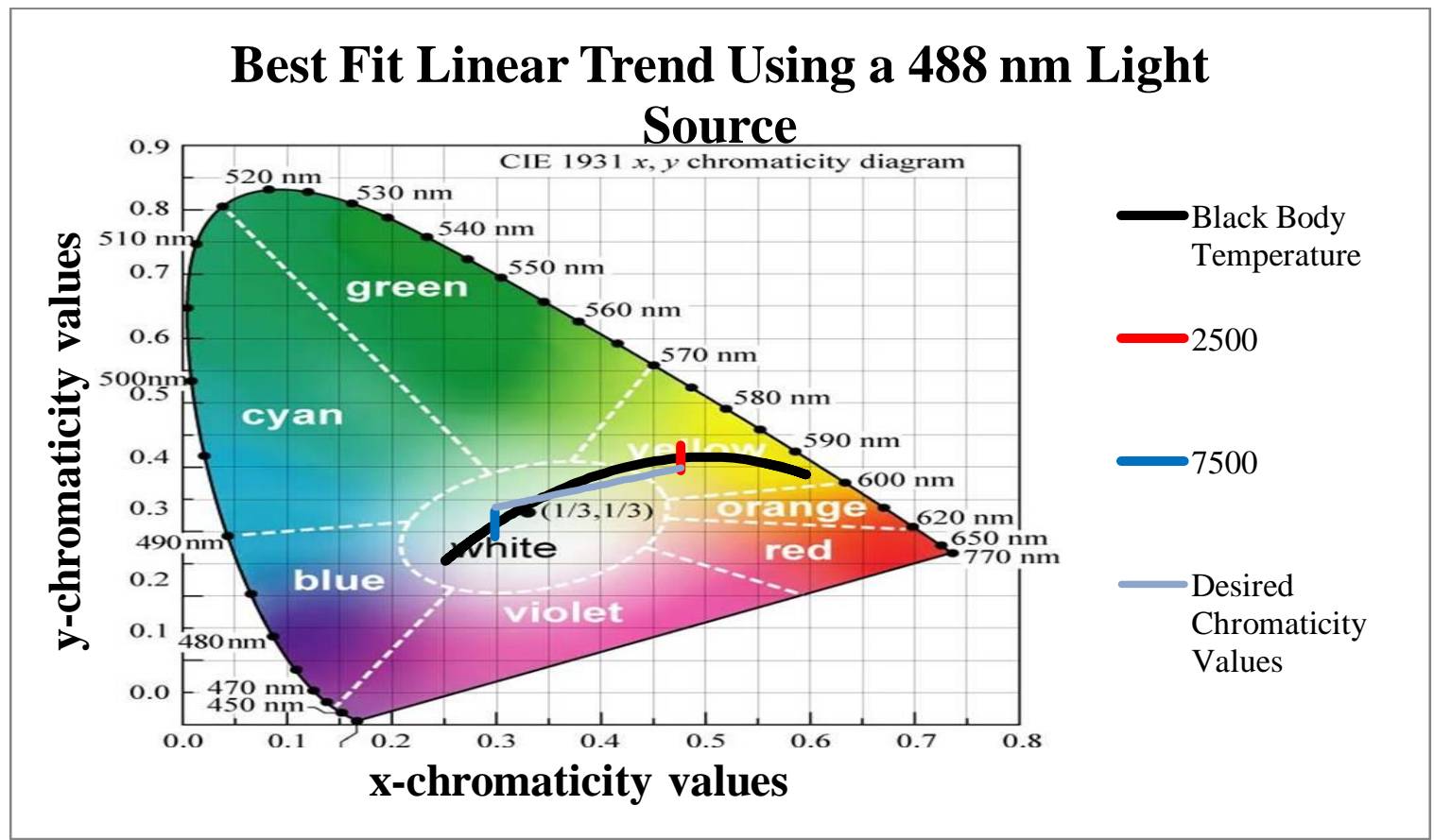

Figure 50 - A linear approximation of the 2500 to 7500 Kelvin temperatures with one light source producing a color value of $488 \mathrm{~nm}$. 
Since the blue phosphor essentially increases the Kelvin value of white light the combination of the blue LED, YAG:Ce, and ZnS:Se must yield the color value at $(0.476$, 0.398).

\section{Crystallograpy and Photoluminescence of BAM:Eu}

Divalent ions such as $\mathrm{Eu}^{2+}, \mathrm{Sm}^{2+}$, and $\mathrm{Yb}^{2+}$ display a $5 \mathrm{~d}$ to $4 \mathrm{f}$ emission similar to YAG:Ce. In the instance of the $\mathrm{Eu}^{2+}$ ion the ground state is the $4 \mathrm{f}^{7}$ configuration. The decay time is near 1 microsecond due to the spin octets and sextets in the emitting level. Since the ground state is an octet $\left({ }^{8} \mathrm{~S}\right)$ the spin selection rule reduces the transition rate. The host lattice has the same effect on $\mathrm{Eu}^{2+}$ as it did on $\mathrm{Ce}^{3+}$. If the crystal field is weak and the amount of covalency is low then it is possible for the $4 f^{6} 5 \mathrm{~d}$ configuration to shift to a higher energy. In that situation the ${ }^{6} \mathrm{P}_{7 / 2}$ level of the $4 \mathrm{f}^{7}$ lies below causing a sharp line in the spectrum to occur from the ${ }^{6} \mathrm{P}_{7 / 2}$ to ${ }^{8} \mathrm{~S}_{7 / 2}$ transition.

The complexity of the BAM:Eu crystal lattice provides europium with two positions to reside in (Figure 51). This in turn creates two distinct energy transitions for an excited electron in the $\mathrm{Eu}$ cloud to transition between. The result is a spectrum not centered about a single wavelength but one which encompasses two peak wavelengths (24). 


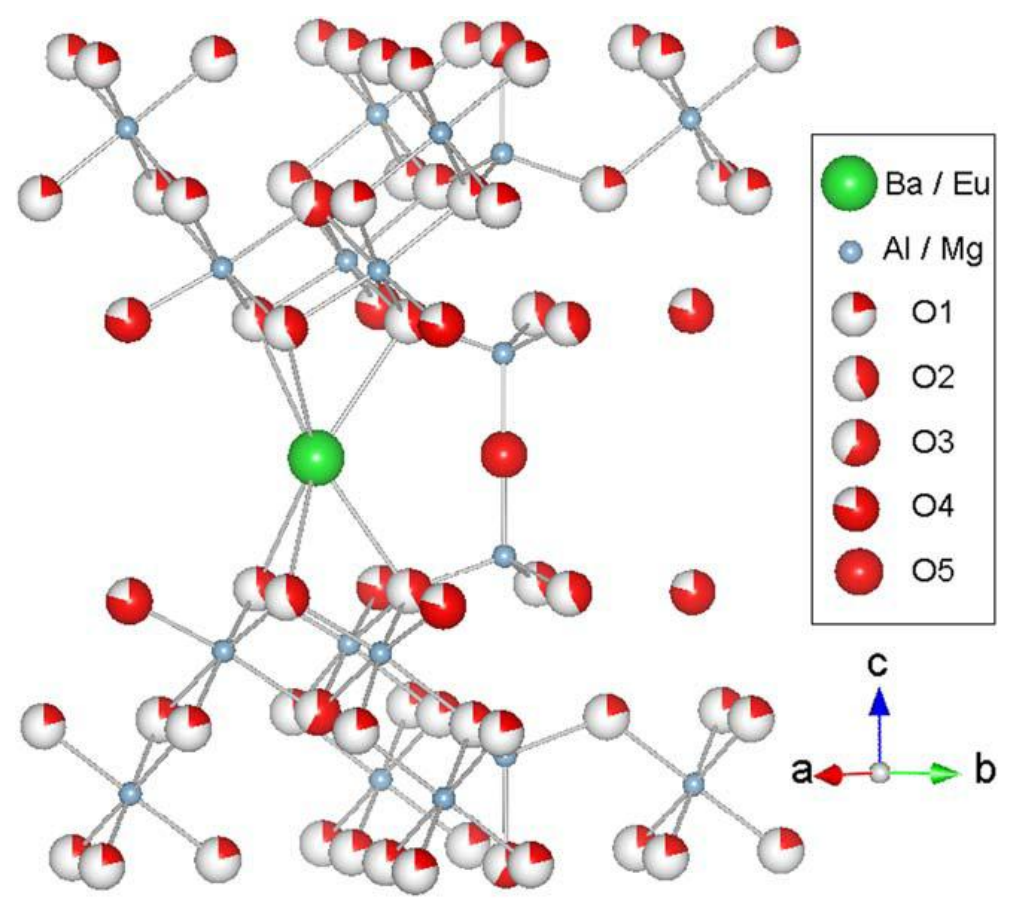

Figure 51 - One half of the complex crystal structure of BAM:Eu. This half shows one of the possible positions for europium to reside in $(43)$.

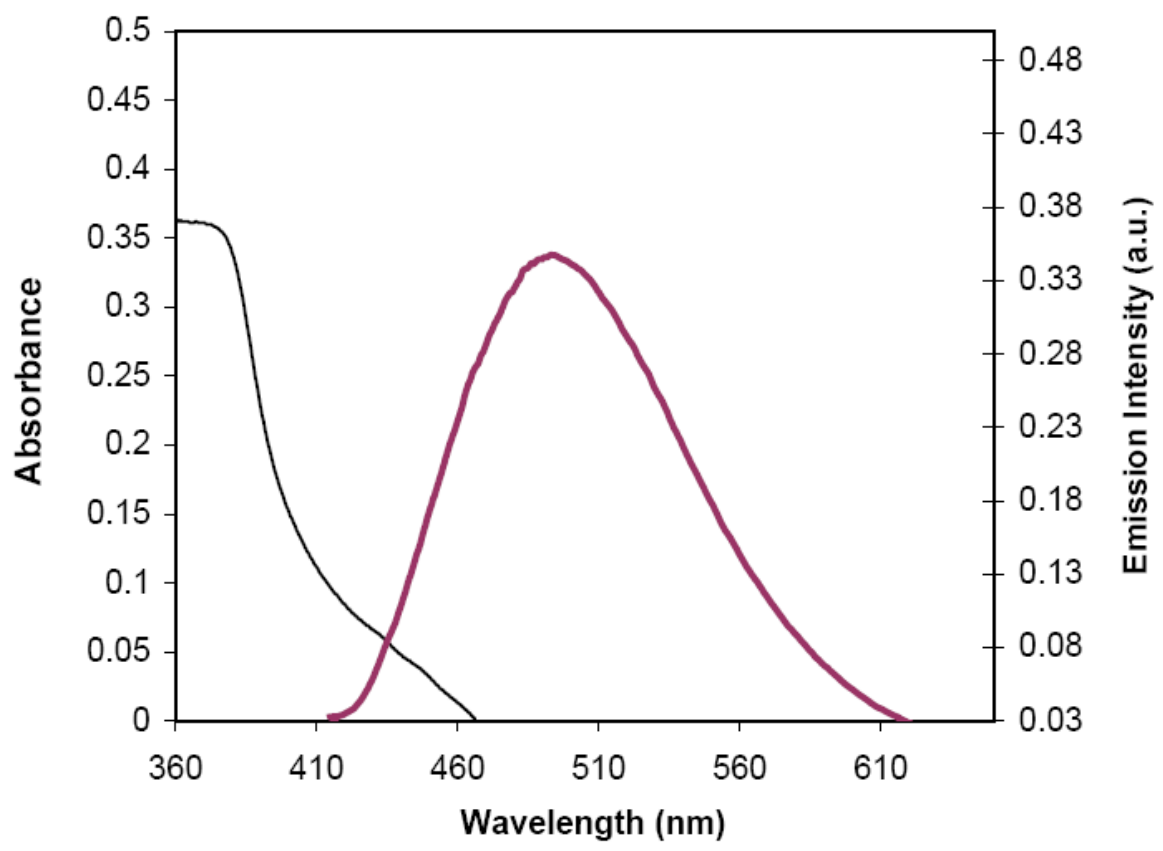

Figure 52 - Absorption and Emission spectrum of BAM:Eu used in this study ${ }_{(44)}$. 
The skewed emission profile of BAM:Eu changed its chromaticity values from what would be expected from a $488 \mathrm{~nm}$ peak wavelength light source. Since light was shifted toward the green region of the spectrum and not narrowly centered around $488 \mathrm{~nm}$ a chromaticity value turned out to be undesirable. Chromaticity values were $x=0.223$ and $\mathrm{y}=0.400$ (cyan colored light) when stimulated with UV light. Multiple tests were performed, all of which indicated that BAM:Eu in conjunction with YAG:Ce, ZnS:Se, and blue and UV LEDs would make for a poor multi-Kelvin white light source. The greatest reason why the phosphor would not be a good choice is its relatively low intensity compared to YAG:Ce and $\mathrm{ZnS}: \mathrm{Se}$. The only method to obtain large changes in chromaticity values would be to have unreasonably low intensities from the other sources of light. Another downside is that BAM:Eu must be closest to the observer relative to the other phosphors. The light emitted from BAM:Eu is at a wavelength which stimulates ZnS:Se and YAG:Ce. If either of those phosphors were between BAM:Eu and the observer their photoluminescence from BAM:Eu emission would be apparent and the color point would not be able to be adjusted. A configuration of that manner prevents the ability to have a multi-Kelvin light source because of the dependence the other phosphors have on BAM:Eu. Since BAM:Eu must be on the outermost layer the UV light is diffused from previous phosphor particles as well as absorbed by the $\mathrm{ZnS}$ :Se (Figure 53). 


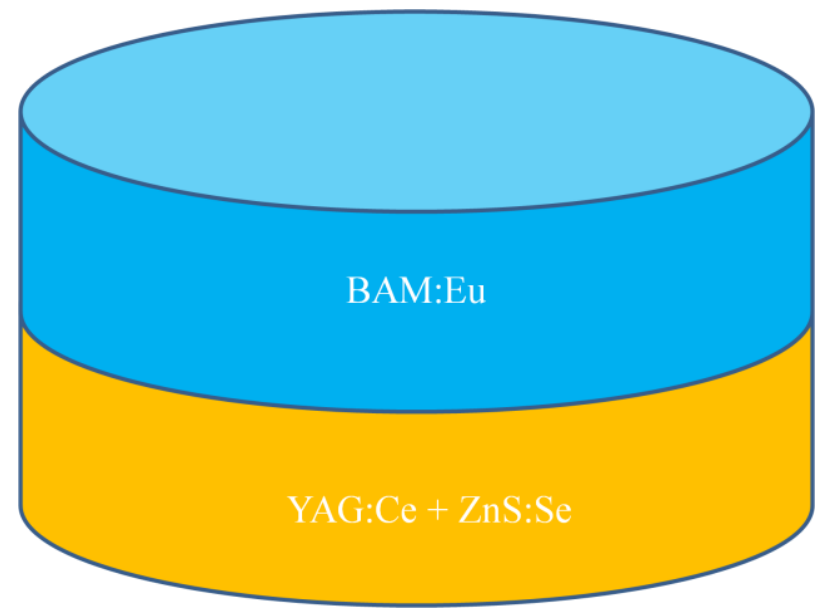

Figure 53 - One of the many configurations of BAM:Eu phosphor in PDMS positioned near PDMS with YAG:Ce and ZnS:Se phosphors. This design requires light to come from the bottom so that an observer (above the phosphors) would not see any stimulation of YAG:Ce or ZnS:Se caused by excited BAM:Eu.

Another option is to allow both the BAM:Eu and the other phosphors to interact with the both LEDs directly so that the UV light could reach BAM:Eu without being diffused first. The largest issue with this design is the fact that blue light from the LED passes through BAM:Eu with only a small amount of loss. Blue light scatters off BAM:Eu particles but does not get absorbed because of its inability to stimulate electrons in the lattice to the next energy level. The intense amount of light which passes through BAM:Eu in that scenario is too great for the limited emission from BAM:Eu to have much of an influence on chromaticity values. The undesirable chromaticity value of BAM:Eu for this test could have been addressed by combining some $\mathrm{ZnS}$ :Se with it but due to the inability to emit a relatively substantial amount of light a multi-Kelvin light source would still not be obtainable (Figure 54). 


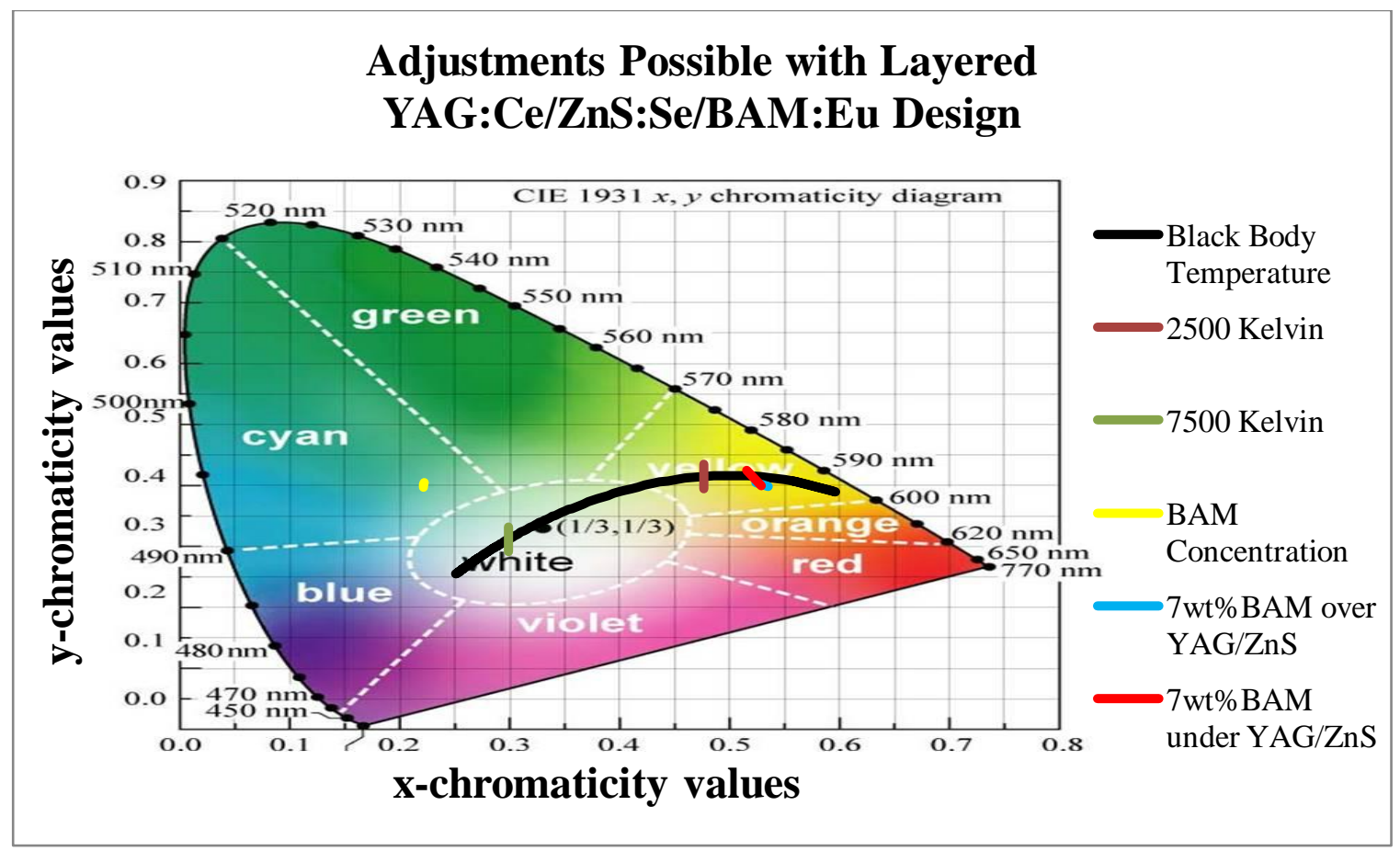

Figure 54 - BAM:Eu produces chromaticity values in the cyan region of this 1931 CIE diagram. When placing BAM:Eu on the LED side of the YAG:Ce and ZnS:Se PDMS substrate, UV excitation causes a blue emission from BAM:Eu which then induces a yellow emission from the YAG:Ce. The result is a more yellow color value. In the opposite orientation, stimulated BAM:Eu would cause a blue-shift but not substantial enough for a multi-Kelvin LED design.

\section{Simulated Spectrum}

Using a $479 \mathrm{~nm}$ blue LED in combination with the $457 \mathrm{~nm}$ LED and the two phosphors, a

spectrum was created to simulate what the blue phosphor was expected to produce

(Figure 55). 


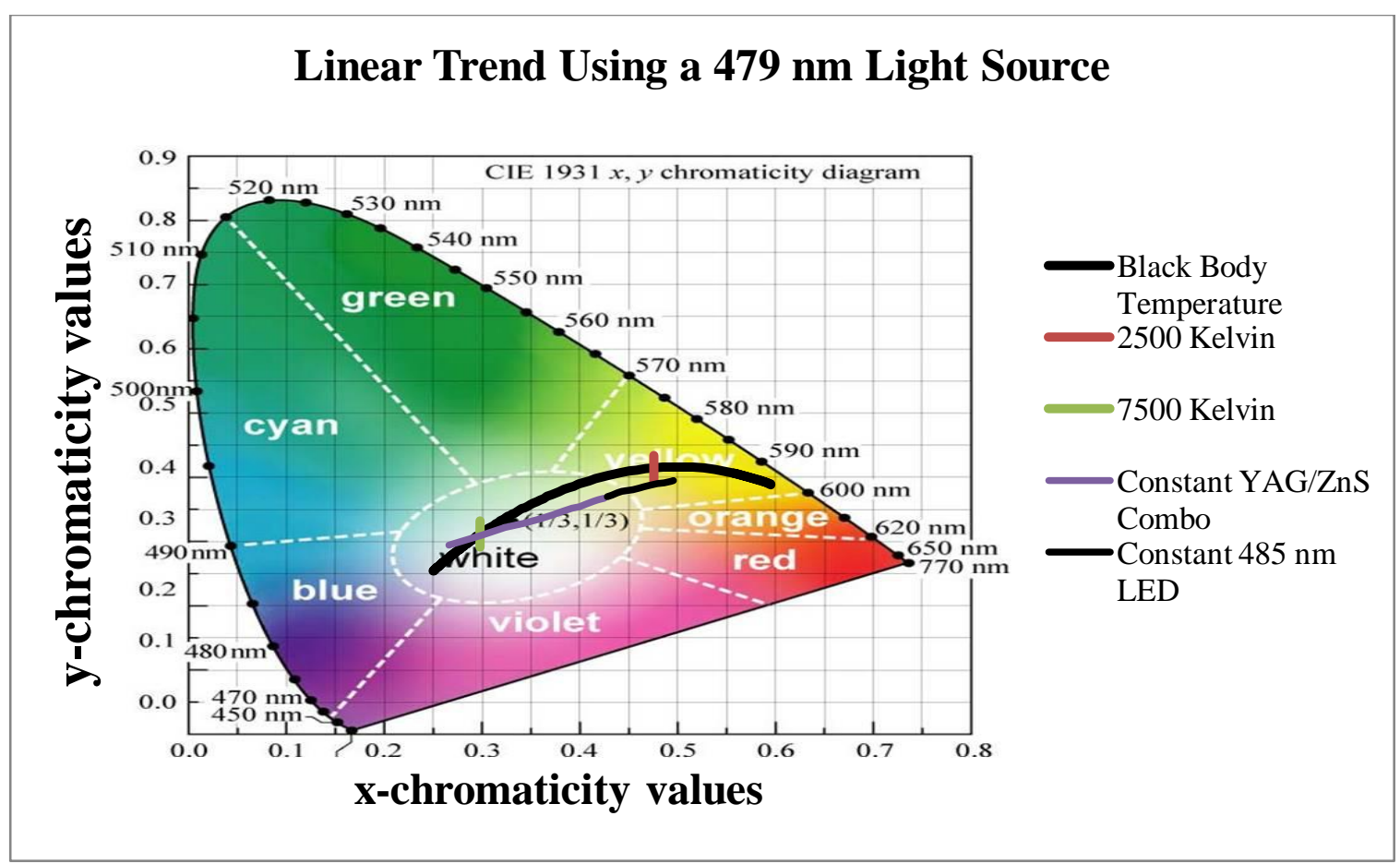

Figure 55 - Actual data from testing completed with a $457 \mathrm{~nm}$ LED and specific concentrations of YAG:Ce and ZnS:Se phosphors illuminated with a $457 \mathrm{~nm}$ LED while a separate $479 \mathrm{~nm}$ blue LED provided additional light to increase the Kelvin temperature. A closer approximation would be possible by adjusting the YAG:Ce and ZnS:Se concentration for that specific $479 \mathrm{~nm}$ blue LED as opposed to the $488 \mathrm{~nm}$ phosphor it was designed for.

The curve was created by holding the 457 blue LED/YAG:Ce/ZnS:Se combo at maximum intensity and incrementally increasing the $479 \mathrm{~nm}$ blue LEDs amperage. With the $479 \mathrm{~nm}$ blue LED amperage maxed the 457 blue LED/YAG:Ce/ZnS:Se combination was incrementally reduced to an intensity which eventually yielded 7500 Kelvin light. The intensities required for these changes will be discussed later. However they are too large to efficiently produce using a UV LED and the $488 \mathrm{~nm}$ blue phosphor. So for the conclusion of this thesis it is proposed to not use a UV LED and instead use a $484 \mathrm{~nm}$ blue LED to provide the large changes in intensity (Figure 56). 


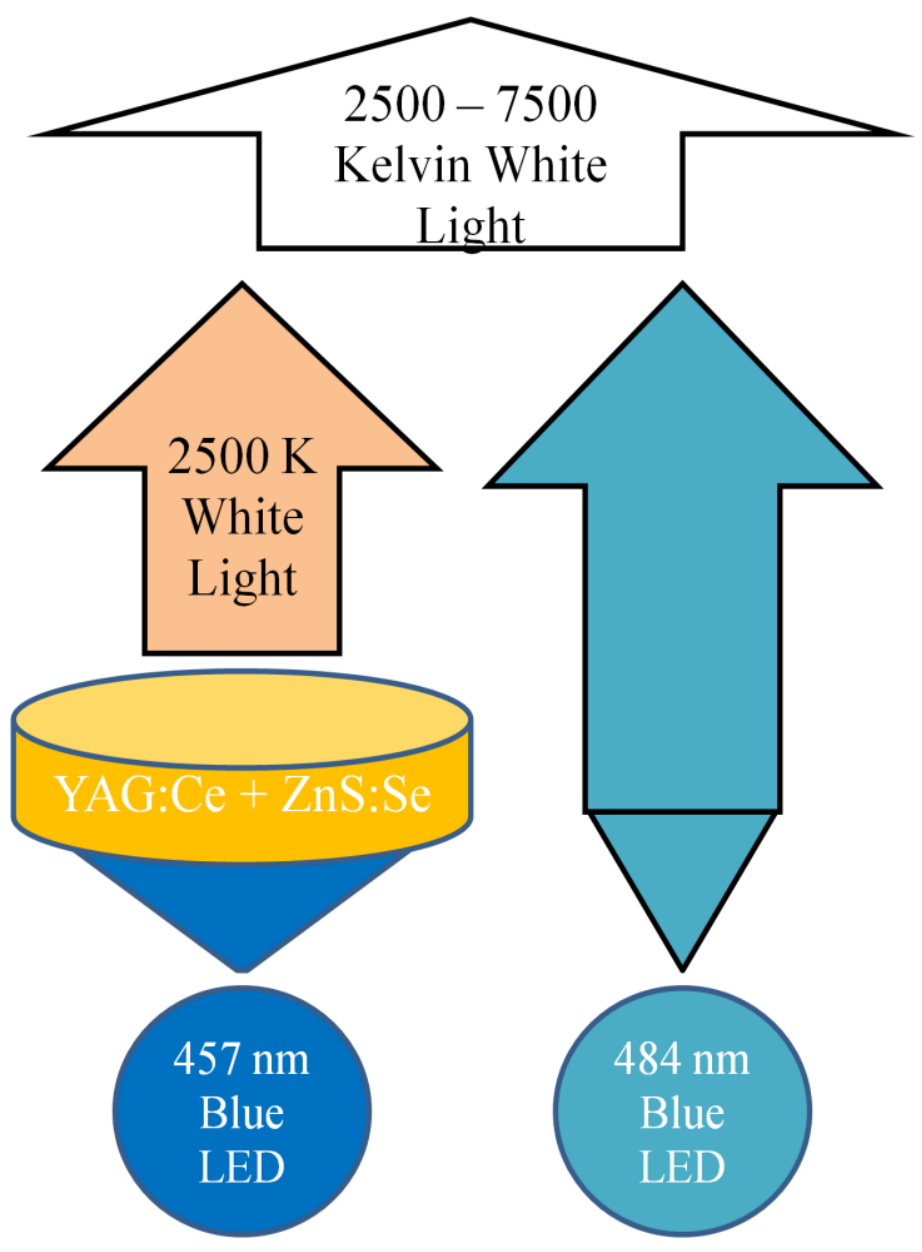

Figure 56 - One possible set up which would yield a white light source that could create Kelvin values between 2500 and 7500 . The $457 \mathrm{~nm}$ blue LED would stimulate a phosphor combination to obtain a color value near 2500 Kelvin on the linear approximation curve while the $484 \mathrm{~nm}$ blue LED provides the necessary intensity to adjust that color value up to 7500 Kelvin.

\section{Phosphor Degradation Issue}

Using a $479 \mathrm{~nm}$ peak wavelength Thorlabs LED in conjunction with a $457 \mathrm{~nm}$ Thorlabs LED plus YAG:Ce/ZnS:Se phosphor in PDMS combination, created a new line to better approximate the Kelvin scale between 2500 and $7500 \mathrm{~K}$ (Figure 57). 


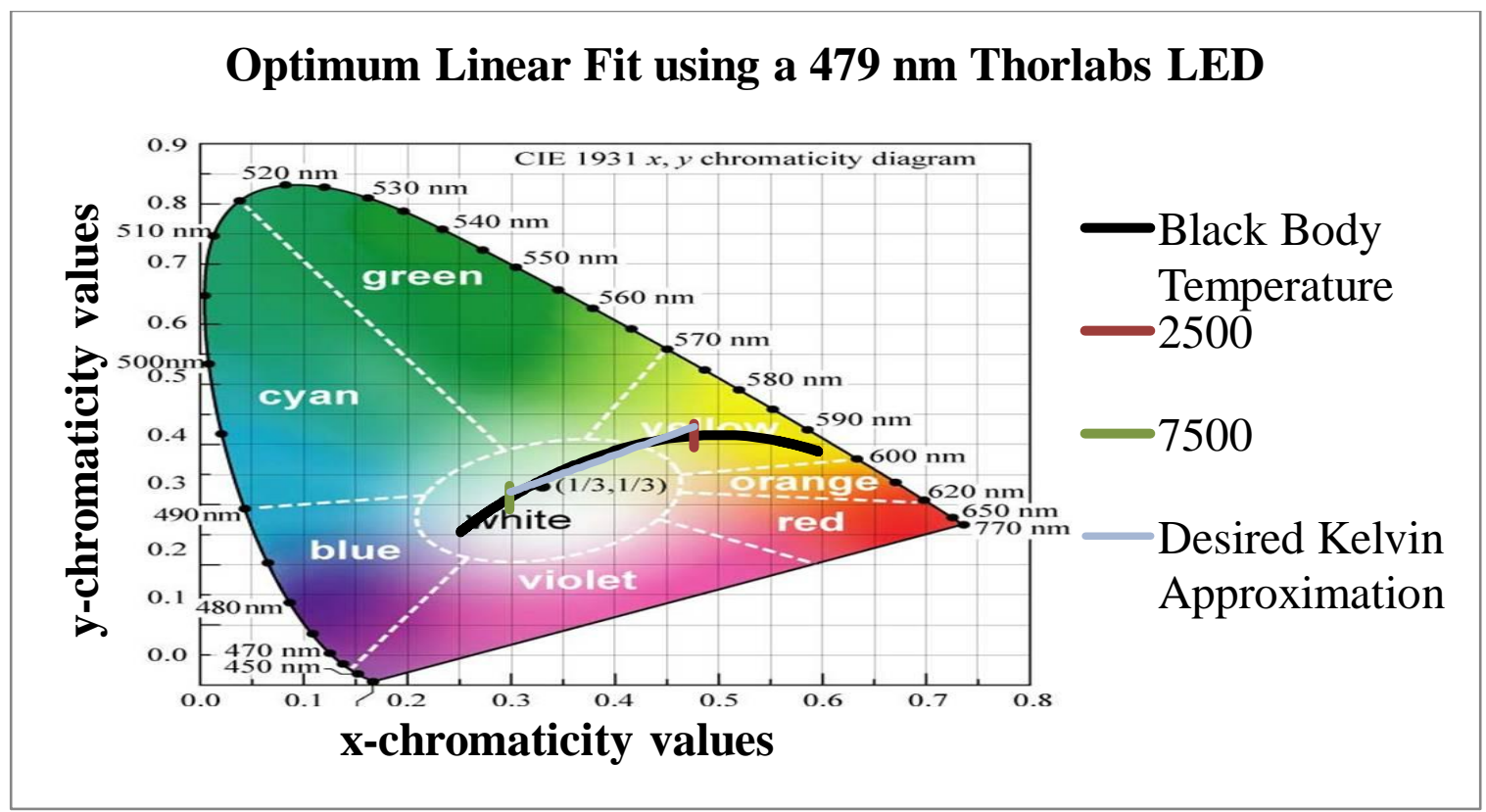

Figure 57 - Displaying the optimum linear approximation of the Kelvin scale between 2500 and 7500 $K$ based off of the chromaticity value for a 479 peak-wavelength Thorlabs LED.

The line with the best fit while using a $479 \mathrm{~nm}$ LED for additional blue stimulation requires a second light source with chromaticity values of $x=0.4765$ and $y=0.4287$. A small design of experiment (DOE) concluded that a $457 \mathrm{~nm}$ LED stimulating the phosphor substrate required a concentration of $3.42 \mathrm{wt} \%$ YAG:Ce and $3.50 \mathrm{wt} \% \mathrm{ZnS}: \mathrm{Se}$ in a $2.50 \mathrm{~mm}$ thick PDMS sample to produce the desired near-2500 K light. The result produced a chromaticity value of $x=0.4785$ and $y=0.4269$. Considering the processing steps of the samples and $10 \mathrm{~nm}$ averaging of spectrums for calculations, those chromaticity values are essentially the same as the calculated values.

Unfortunately, degradation with the phosphor during testing caused shifts in chromaticity values prior to performing an experiment. As soon as the phosphor substrate was excited from the blue LED at maximum current a decline in peak phosphor intensity was 
observed. This decline decreased rapidly over the first few minutes and began to come to equilibrium around ten minutes (Figures58 and 59).

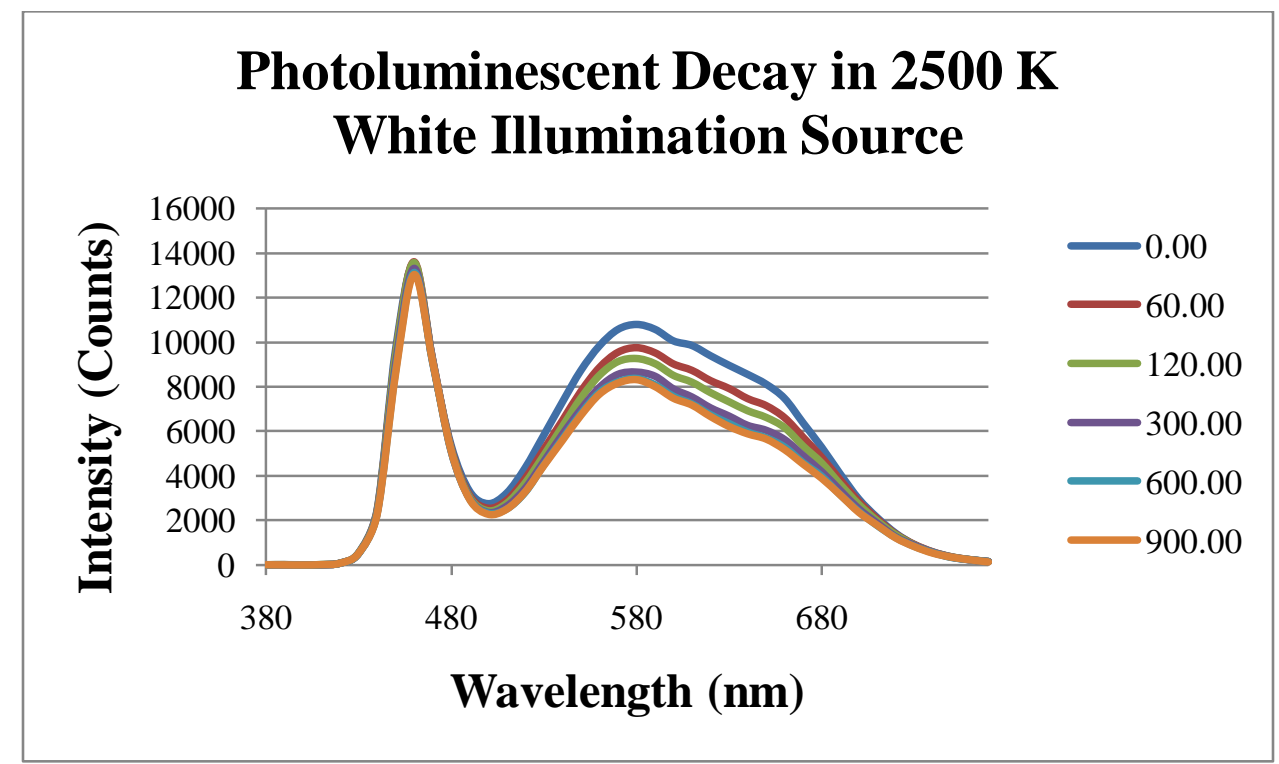

Figure 58 - A significant decrease in photoluminescent intensity over a 900 second (15 minute) interval. The intensity of the $457 \mathrm{~nm}$ blue Thorlabs LED remains essentially unchanged throughout this time period. 


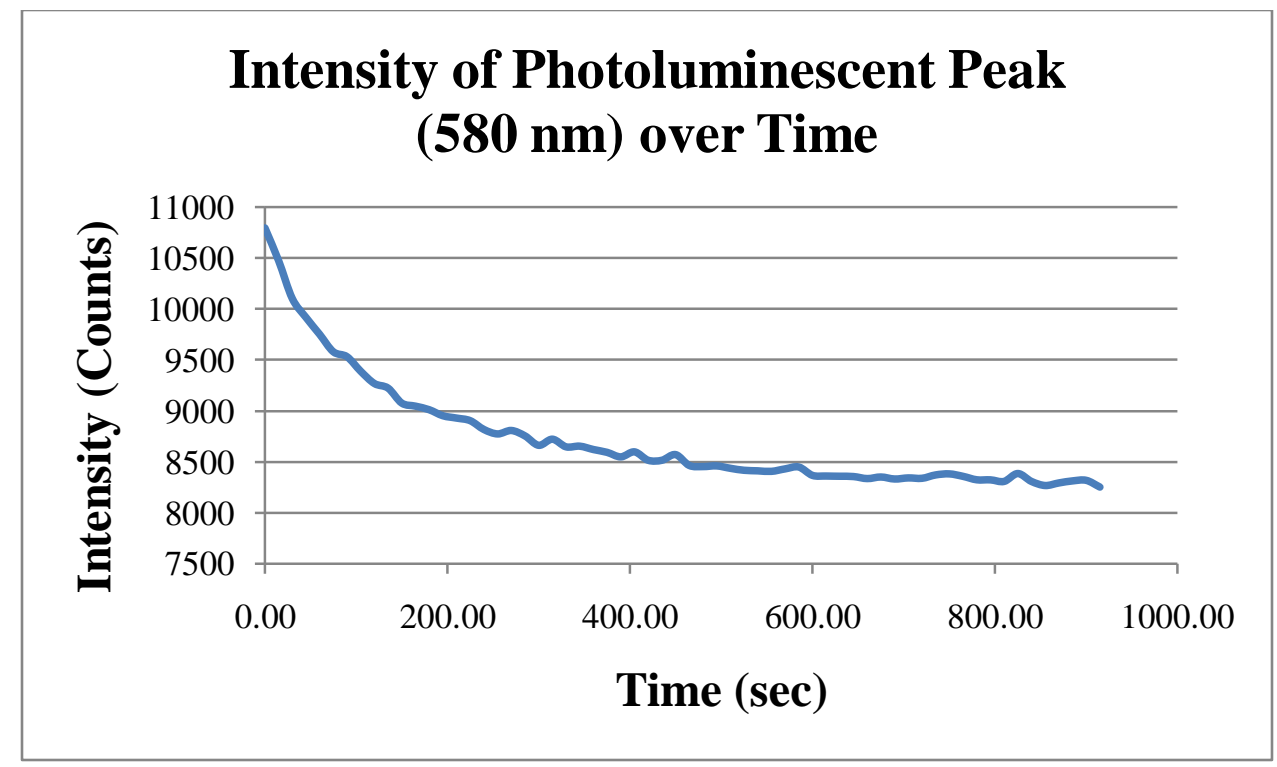

Figure 59 - The peak intensity of the photoluminescent region, comprised of YAG:Ce and ZnS:Se, throughout a 900 second interval. The decay appears to reach a plateau around 10 minutes of exposure to the $457 \mathrm{~nm}$ Thorlabs LED at $670 \mathrm{~mA}$.

It is postulated that the decrease in phosphor emission was caused by an intensity decay of the $\mathrm{ZnS}$ :Se. That particular inorganic phosphor photoluminesces when electrons decay into defects such as vacancies, interstitials, and additional states provided by surface atoms. In the final lens configuration, $\mathrm{ZnS}: \mathrm{Se}$ is housed in a silicone oil film that is trapped in position by cured PDMS. This may lead to unsatisfied zinc bonds on the $\mathrm{ZnS}:$ Se particle surface to become saturated with the silicone oil. Studies have shown the susceptibility of $\mathrm{ZnS}$ to oxygen adsorption by photoinduced methods. In $\mathrm{ZnS}$ powders, adsorption has been found to proceed via chemisorption by thermal or radiative processes (45). Oxygen atoms combine with zinc to form a nonluminescent center with $\mathrm{Zn}-\mathrm{O}-\mathrm{Si}$ bonds on the phosphor particles surface (46). Over time this reaction repeats with many surface atoms. It has been shown that the formation of $\mathrm{ZnO}$ on the surface of a $\mathrm{ZnS}$ phosphor can decrease luminescence (47). This mechanism not only forms a cap on the 
$\mathrm{ZnS}:$ Se particles but also satisfies some of the important atomic states for photoluminescence on the surface. Since this coating is likely not uniform or of appropriate thickness it does not improve luminescence as some transparent coatings are tailored to do. Without the additional states on the surface, photoluminescence decreases as there are fewer electron-hole pairs for electronic transitions to occur (48). Photoluminescence quenching, photoinduced oxygen adsorption, and photodecomposition are sensitive to surface effects with $\mathrm{ZnS}$ particles (49).

This change in phosphor emission intensity created unexpected adjustments in chromaticity values. Determining chromaticity values of samples were conducted by quickly exposing the phosphor to the blue LED and saving a spectrum. Deviations were found to occur between chromaticity values one day after sample creation and 16 days (day of multi-Kelvin source testing) after sample creation. Between nine samples this deviation appeared random so as to not indicate a specific trend in chromaticity values due to time from fabrication. However, when testing the multi-Kelvin ability of a particular phosphor/PDMS sample a large shift in the negative $\mathrm{x}$-chromaticity direction occurred. The time necessary to perform amperage adjustments to the various LEDs and record spectrums was enough for the measured color value to shift far from the desired (and previously measured) 2500 Kelvin value to some region near 5000 Kelvin white light. Due to this behavior a different sample (3.31 wt $\%$ YAG:Ce and $3.45 \mathrm{wt} \% \mathrm{ZnS}: \mathrm{Se}$ in a $2.50 \mathrm{~mm}$ thick substrate) was used to mimic the Kelvin scale (Figure 60). The test was conducted as quickly as possible to negate issues with phosphor degradation. 


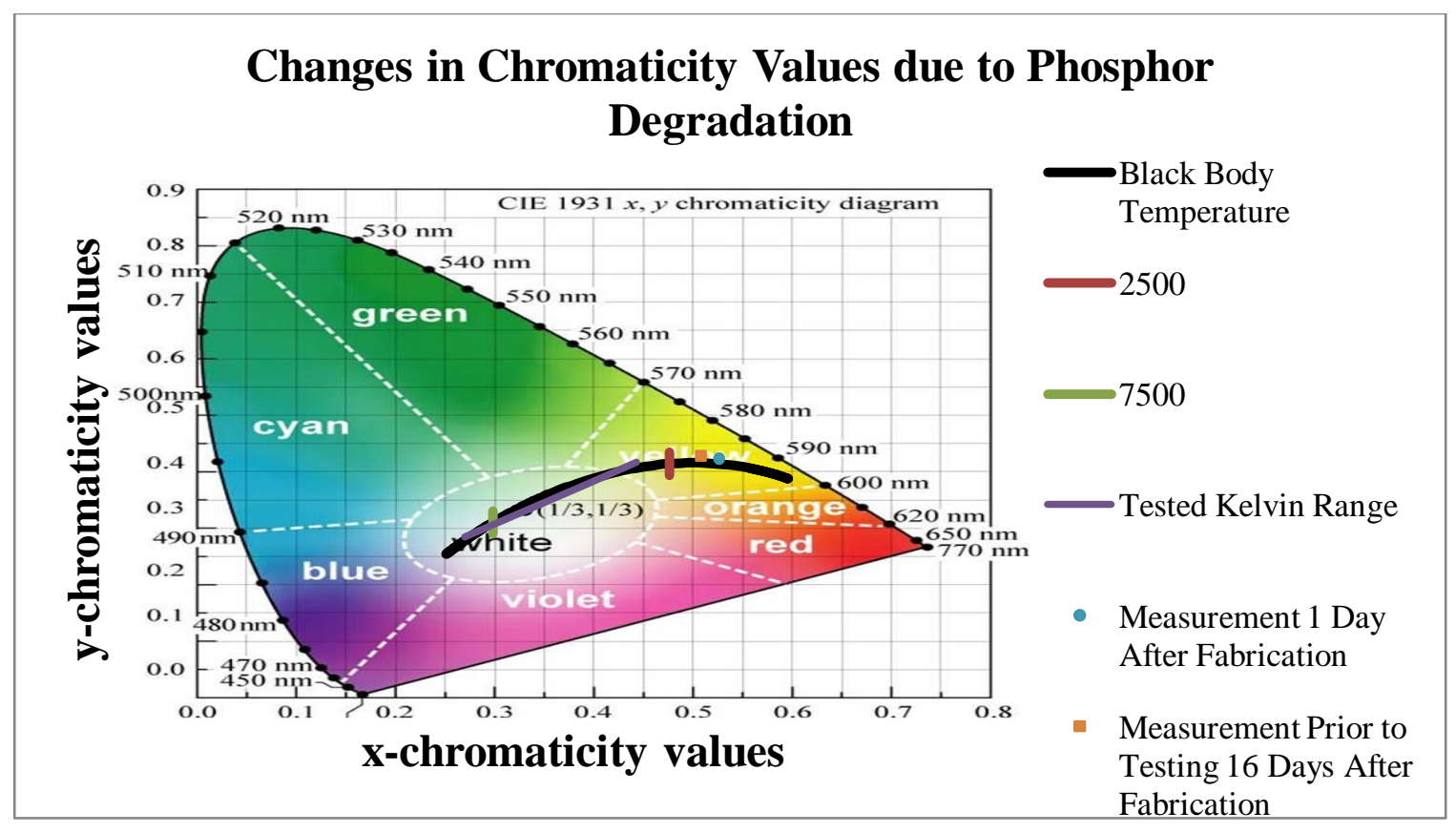

Figure 60 - Due to the degradation of $\mathrm{ZnS}$ :Se phosphor the closest approximation to the specific intervals desired on the Kelvin scale deviated from the calculated fit. Prior to prolonged blue light exposure the chromaticity values of this substrate were more yellow than $2500 \mathrm{~K}$.

The range of chromaticity values are possible when the light source is initially turned on but over a ten minute interval they will rapidly shift in the direction of the $479 \mathrm{~nm}$ blue LED chromaticity color. 


\section{CHAPTER VIII: CONSIDERATION OF CHROMATICITY GRADIENTS AND THE INTENSITY RELATIONSHIP WITH KELVIN VALUES OF THE FINAL DESIGN}

\section{Introduction}

There are two major problems necessary to address for a functioning multi-Kelvin white

LED. One issue being the color gradient across the PDMS/Phosphor surface, ignored because of the beam splitter and integrating sphere testing configuration. The other issue is that the intensity adjustments necessary to obtain all values along the Kelvin scale are significant. Meaning that the brightest the light will be is near 3100 Kelvin and the dimmest the light will be is at 7500 Kelvin.

\section{Color Gradient}

The color gradient may simply be addressed by producing a surface which has varying thicknesses (Figure 61). The configuration of the few data points in addition to the symmetry about the center point led to the use of a Gaussian distribution to fit a model 


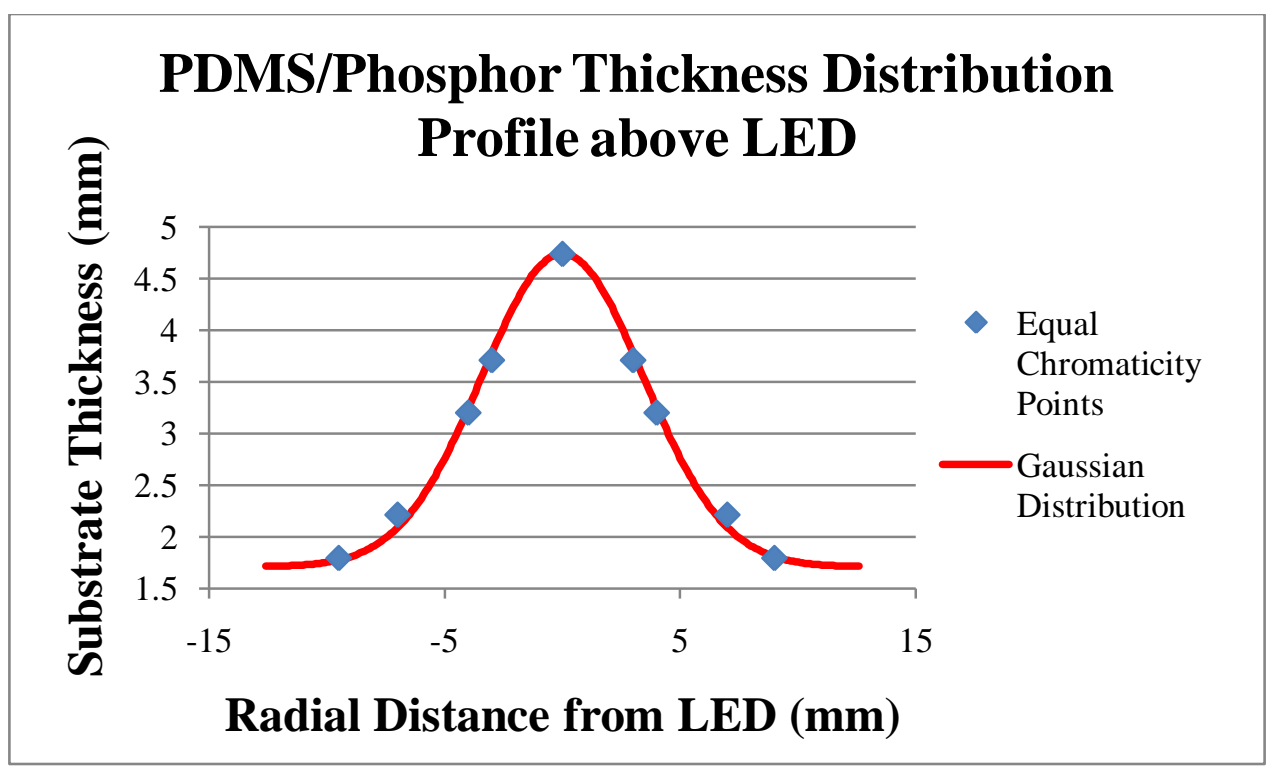

Figure 61 - Data from a previous study indicating what thickness of a phosphor substrate is required to produce the same chromaticity values. A specific Gaussian distribution would be required for a given LED configuration.

The highest point of the Gaussian surface should be positioned directly above the LED and thinned out with increasing radial distance from the LED. Each Gaussian curve is dependent on the LED set up (i.e. area the LEDs take up for a given group of die and the distance they sit below the phosphor substrate). To test this theory a one inch lens was designed in Solid Works using data from the above Gaussian curve (Figure 62).

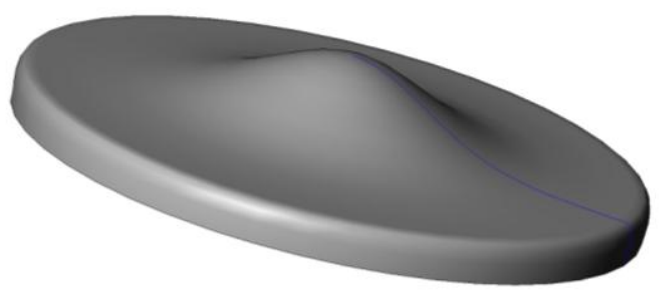

Figure 62 - SolidWorks depiction of a one-inch diameter disk with a peak thickness of $4.74 \mathrm{~mm}$ and a minimal thickness of $1.71 \mathrm{~mm}$. 
The lens was manufactured using the pouring method, described previously, into a mold created by rapid prototyping (Figure 63).

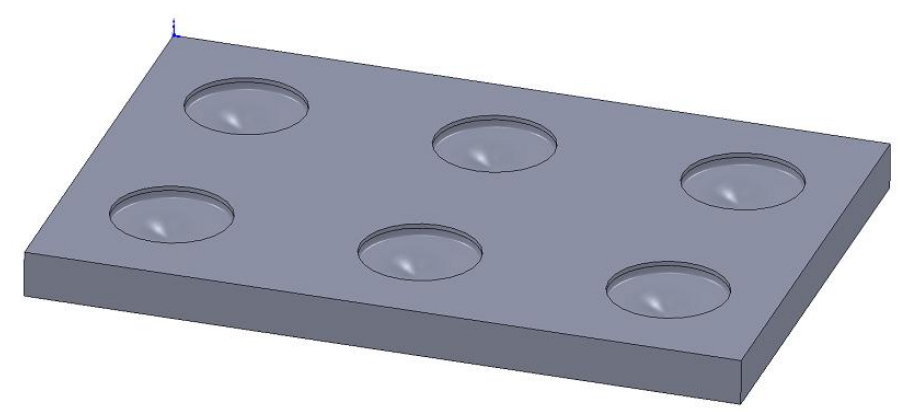

Figure 63 - A model of the casting tray created by Z-Corp rapid prototyping.

To create a structure with the dimensions of $1.71 \mathrm{~mm}$ at the thinnest regions and $4.74 \mathrm{~mm}$ at the peak, 1.0877 grams of phosphor/PDMS were used. Two samples, one a constant $1.53 \mathrm{~mm}$ and the other a Gaussian profile with edge thickness of $1.53 \mathrm{~mm}$, were created with 3.44 wt\% YAG:Ce for the uniformity tests. A DOE was developed to determine the influence of radial distance, and intensity on chromaticity values. Radial distance was determined with the $\mathrm{x}$ and $\mathrm{y}$ coordinates of the linear motion controller from the center of the most intense region (portion directly above the $457 \mathrm{~nm}$ blue LED). Intensity was characterized by power levels (4.49 Watts, 3.58 Watts, and 1.51 Watts) to the LED set up for each test.

Chromaticity values decreased with an increase in radial distance in the Gaussian substrate (Figure 64). 


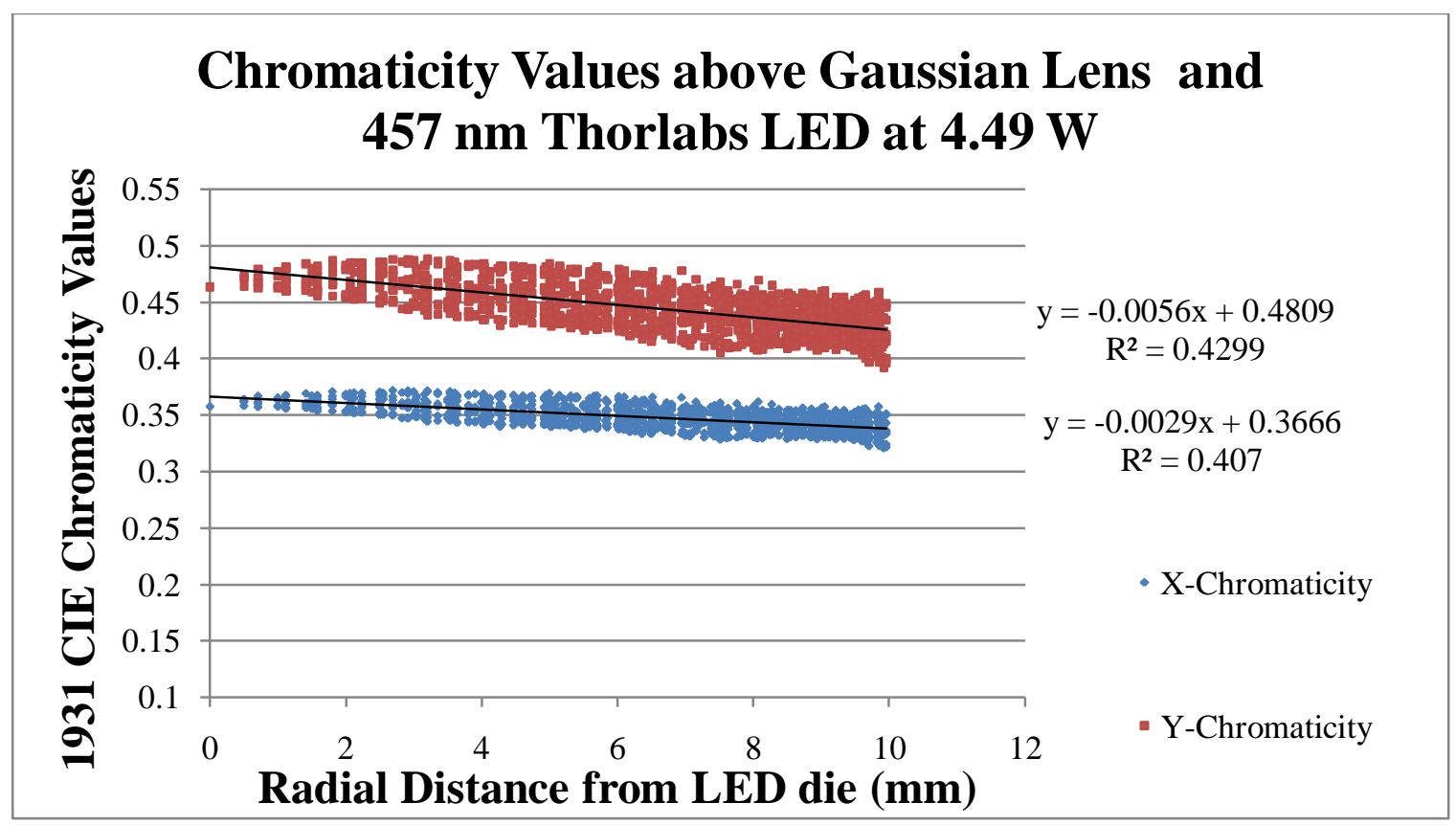

Figure 64 - Measured chromaticity values on a Gaussian surface at various radii from the LED center.

The decreasing chromaticity trend implies that the thickness in the center was too thick for the concentration utilized for this study. A decrease in concentration may address this problem but more testing would be necessary to confirm. Since YAG:Ce phosphor dispersed in PDMS and a $457 \mathrm{~nm}$ blue LED were the only color sources, a high chromaticity value implies more yellow light and a low chromaticity value implies more blue light. In this particular study $\mathrm{ZnS}$ :Se was not used in order to minimize the factors contributing to chromaticity changes. Decreasing the intensity improved uniformity but at the cost of decreased luminous emittance especially at radii greater than $9 \mathrm{~mm}$ (Appendix $\mathrm{X}$ and $\mathrm{Y}$ ). With a constant thickness substrate, as most tests throughout this study have been, the chromaticity values increased with increasing radial distance (Figure $65)$. 


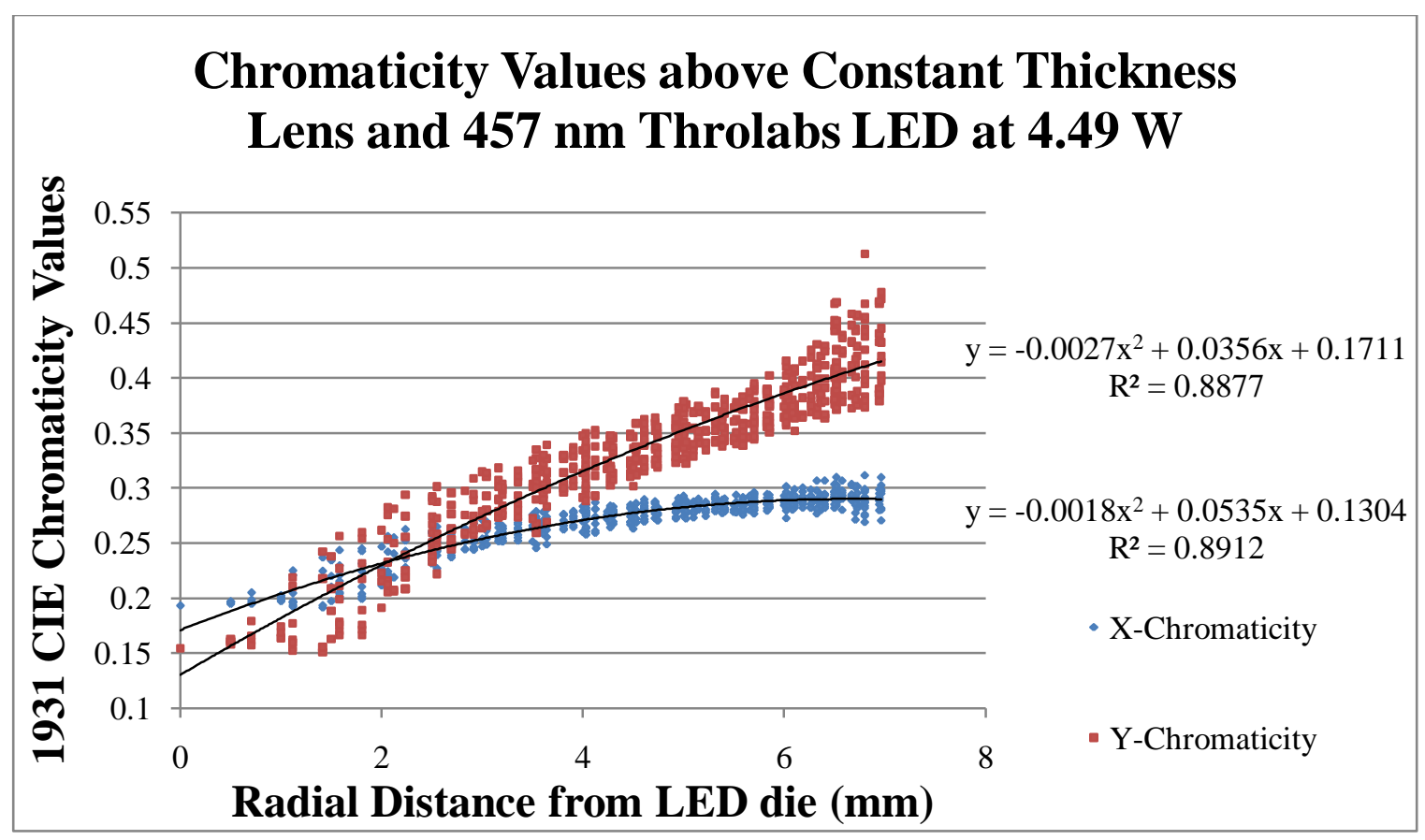

Figure 65 - Above a flat phosphor/PDMS surface chromaticity values changed dramatically with changes in radii from the LED center. This translates to a poor white LED illumination source.

This is due to the intensity of blue light from the LED which has greater transmittance through the phosphor substrate in the center because of the die position. In addition to the increasing trend the variation was much higher (Figure 66). 


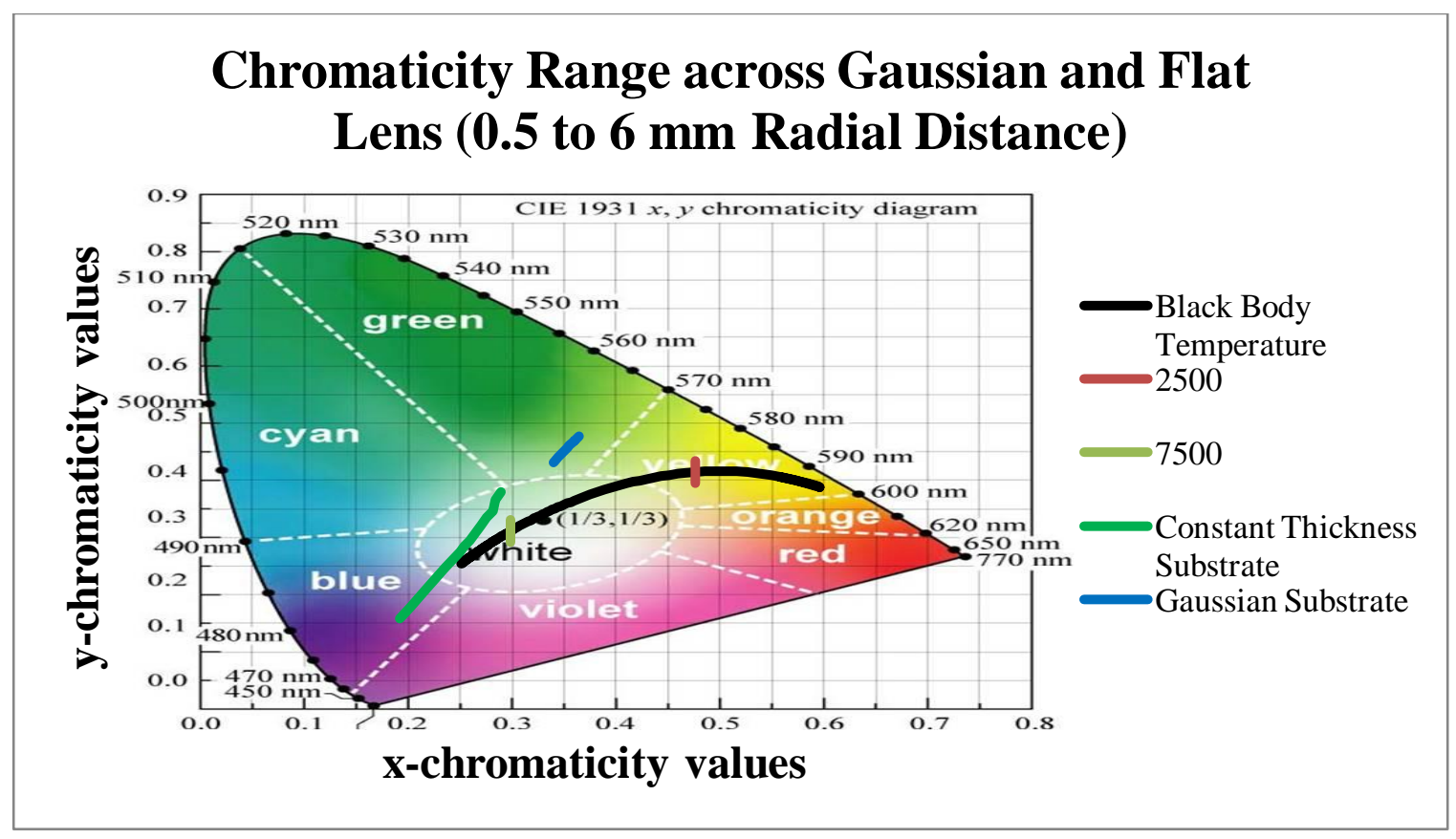

Figure 66 - The chromaticity deviation at various radii was large in the constant thickness sample as expected based on previous tests. The Gaussian profile sample had much less of a deviation over the same radial distance.

Not only did the Gaussian profile reveal more uniform color over the same radial interval, intensity was also found to be more evenly distributed. The flat substrate had measurable (above noise limits of the spectrometer) light production out to a radius of 7.5 $\mathrm{mm}$ where as the Gaussian profile continued to provide useful illumination out to the edges of the Thorlabs cylinder housing the lens $(12.5 \mathrm{~mm})$. This is apparent by comparing the width of the intensity curve based on radial distance (Figure 67 and 68). 


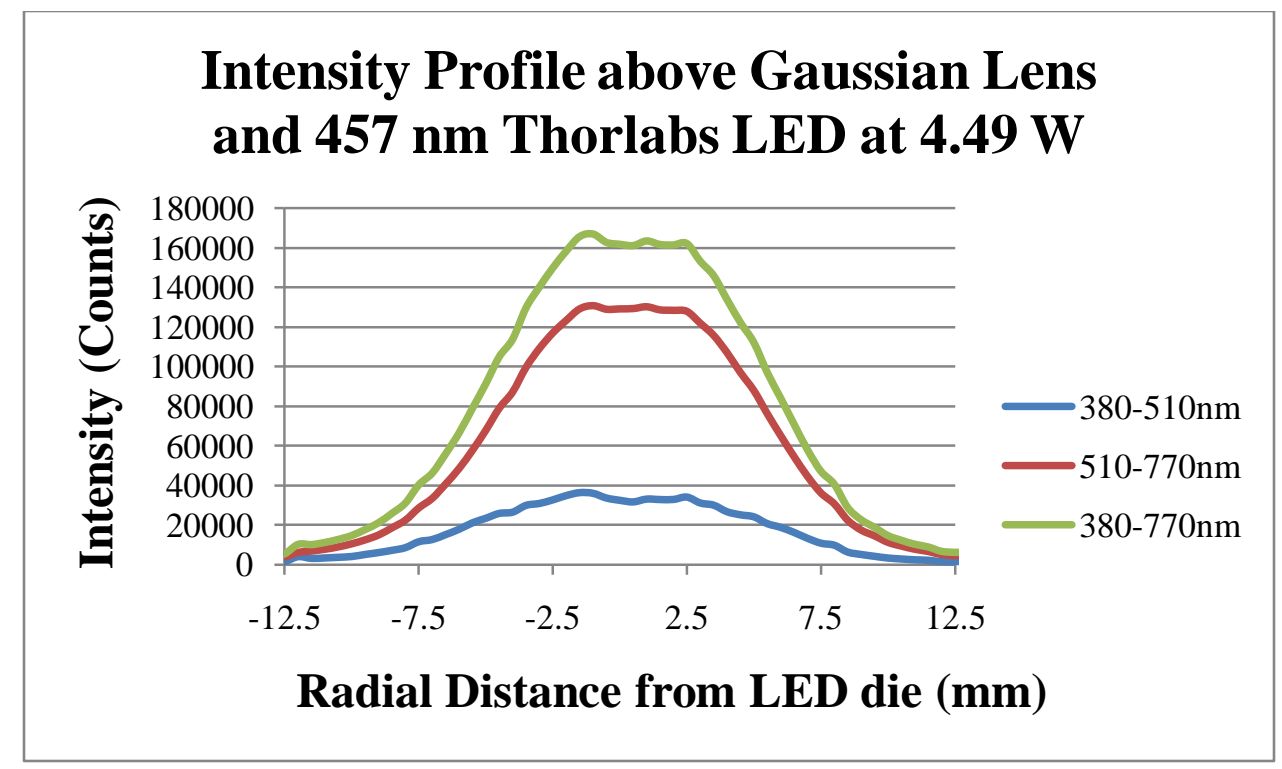

Figure 67 - Intensity above the Gaussian lens still displays more counts directly above the LED than at the outer edges but the design did improve over a constant thickness lens.

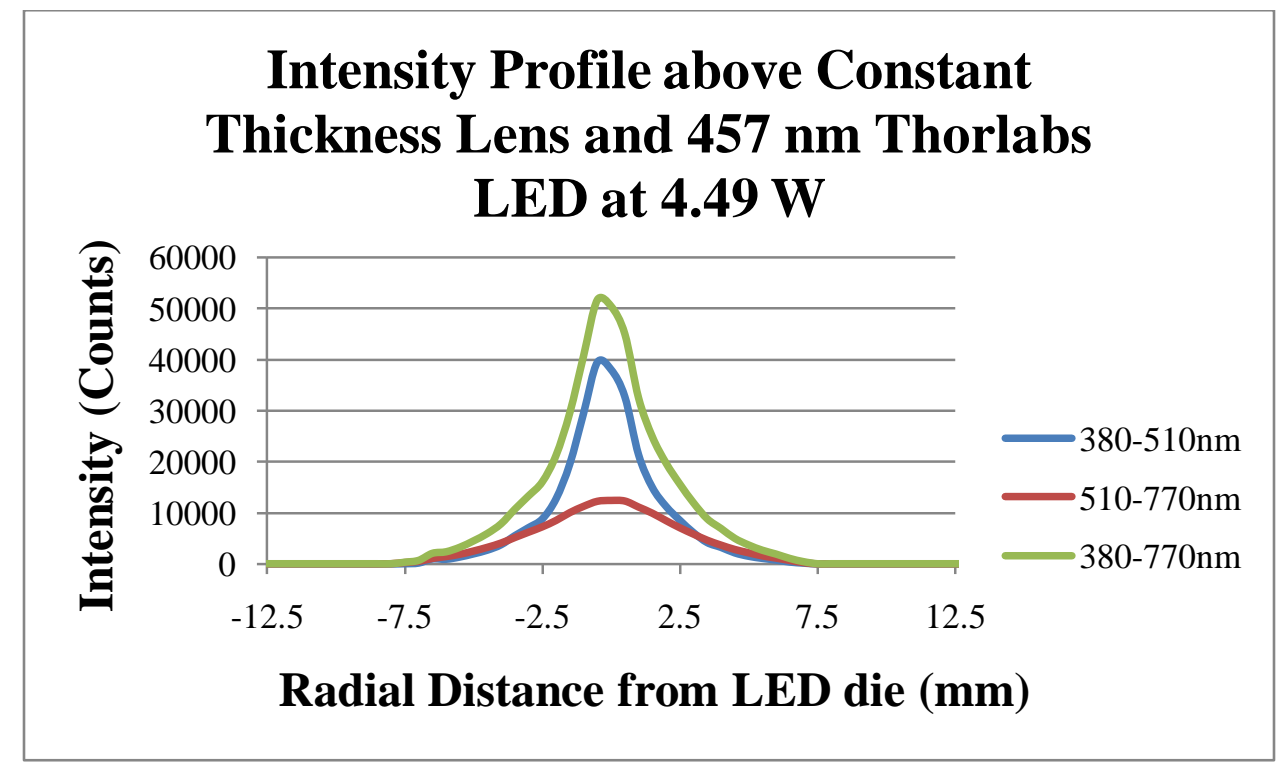

Figure 68 - In addition to the large intensity gradient with radii, blue (380-510 nm) wavelengths are shown to dominate the spectrum at lower radii and decrease below photoluminescent wavelengths $(510-770 \mathrm{~nm})$ which is the cause for the large color gradient observed in constant thickness phosphor/PDMS lenses.

As described with the chromaticity plots versus radial distance, the Gaussian lens displays a much less dramatic change in color over the entire radius. This is also 
apparent by noting the change in dominant luminescent peak found when measuring the constant thickness substrate (Figure 68). At approximately $4 \mathrm{~mm}$ away from the LED die center, photoluminescence dominates the spectrum relative to measured LED emission. This shift in dominate emission source does not occur in the Gaussian lens which is why chromaticity values do not vary with the same magnitude.

\section{Intensity Issue}

The intensity issue may ultimately limit the range of light desired from this kind of light source. The following three graphs display the spectrums which produce Kelvin values between 2100 and 7400 (Figures 69, 70, and 71).

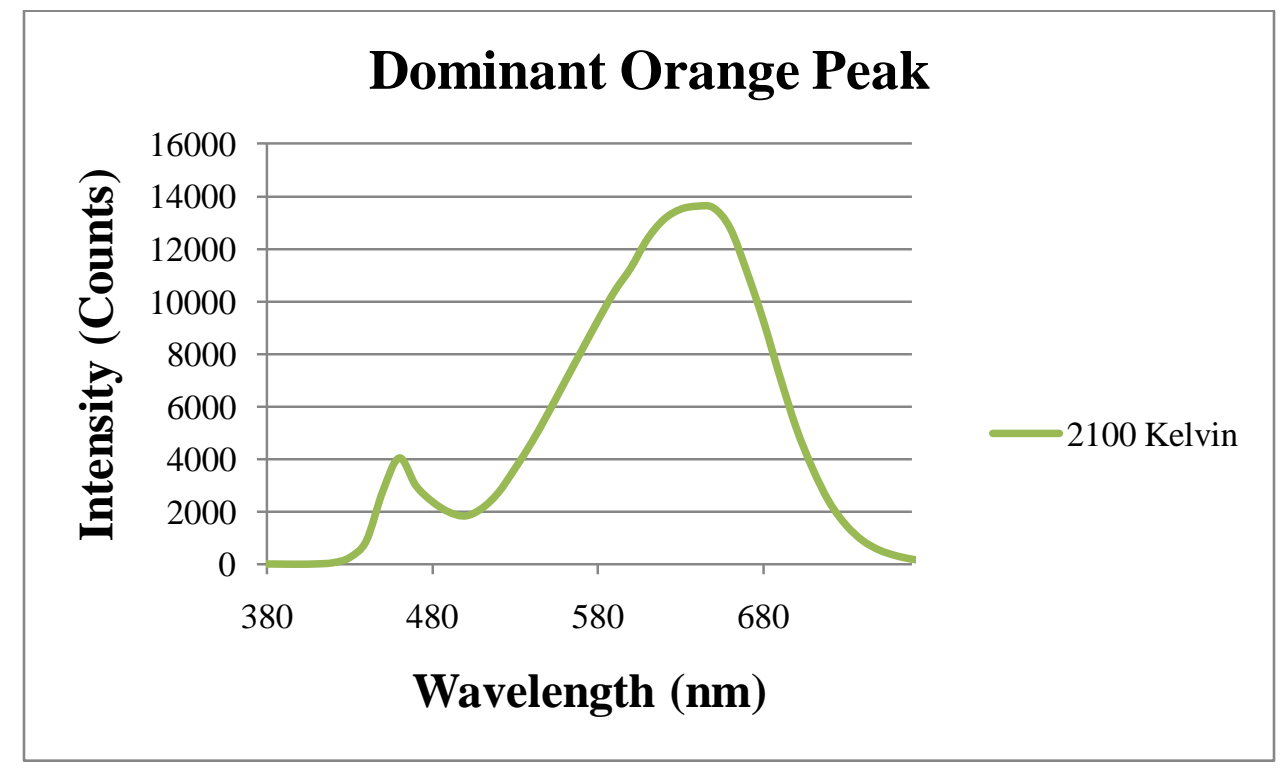

Figure 69 - To produce warm light a spectrum dominated by YAG:Ce and ZnS:Se luminescence is required. 


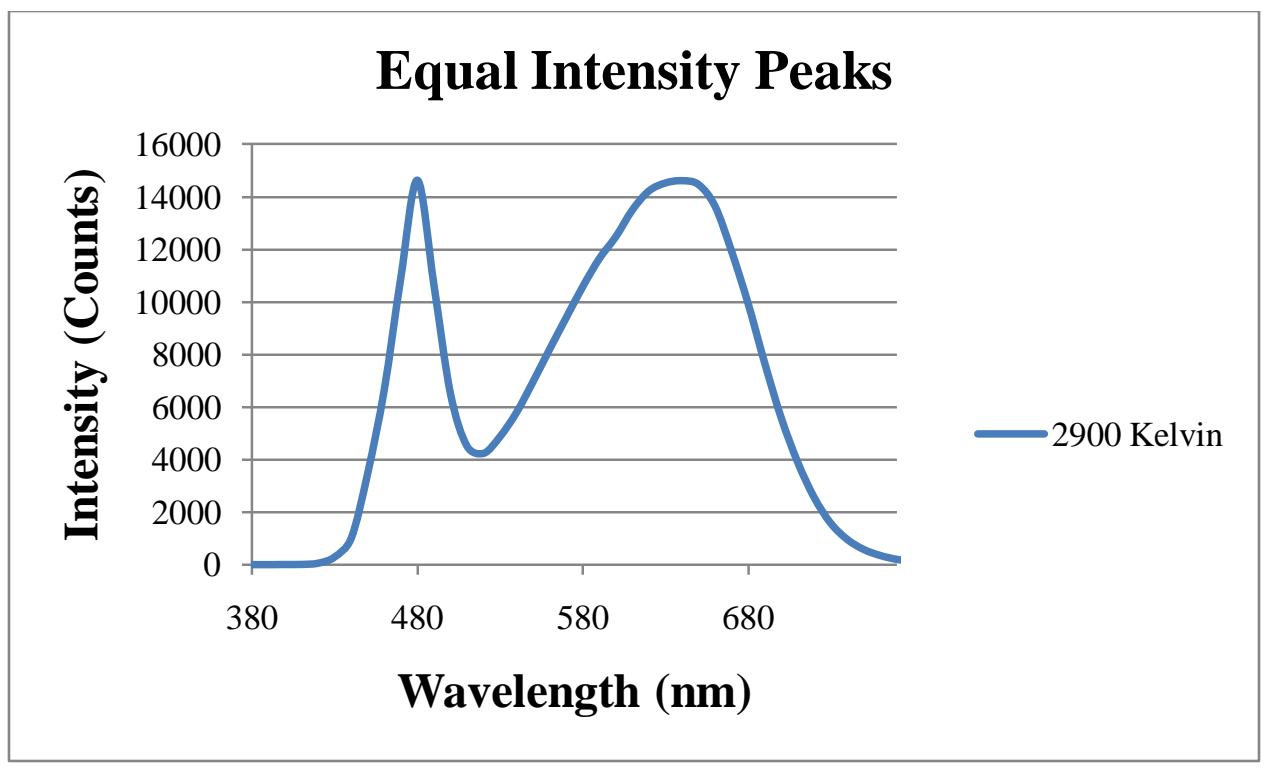

Figure 70 - With the $479 \mathrm{~nm}$ LED, $457 \mathrm{~nm}$ LED, YAG:Ce, and ZnS:Se equal peak intensities (brightest the bulb would be) is at 2900 Kelvin and would shift depending upon the LED wavelengths and phosphor concentration.

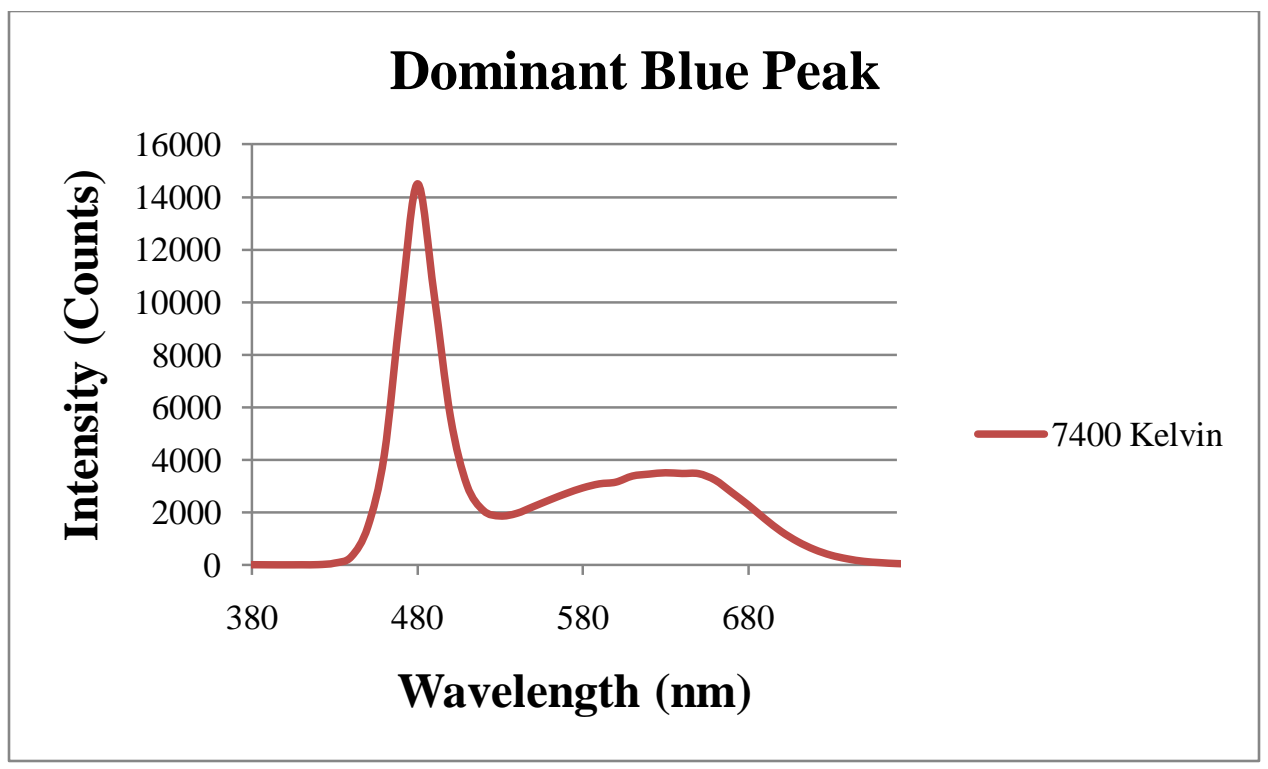

Figure 71 - To produce cool-white light the spectrum must be dominated with the $479 \mathrm{~nm}$ LED light and a significantly less phosphor contribution. This curve would be the dimmest of all the colors on the Kelvin approximation curve. 
The total intensity at various Kelvin values would need to be investigated to determine if the light output is significant for the specific times of day for which that color would be desired (Figure 72).

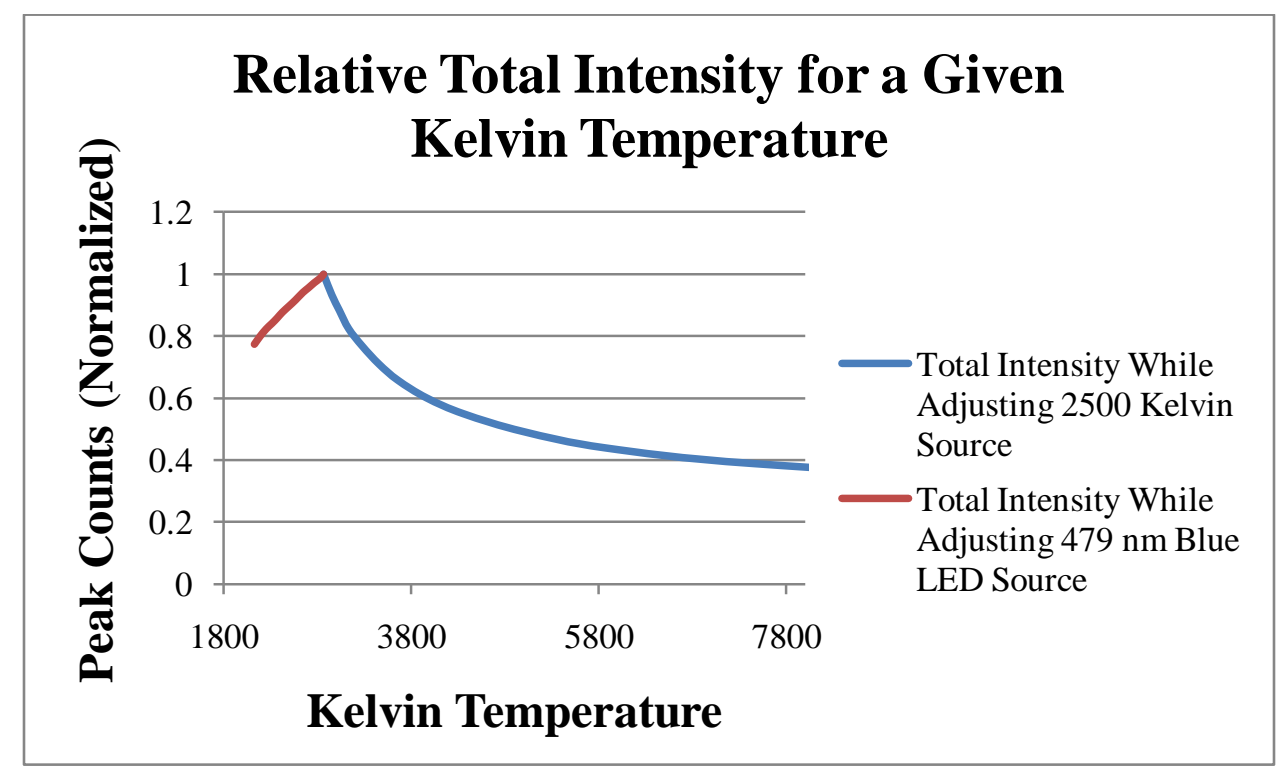

Figure 72 - Normalized intensity of light at a given Kelvin temperature. The red curve is changed while holding the $457 \mathrm{~nm}$ LED/YAG:Ce/ZnS:Se combination intensity constant. The blue curve was the result while holding the $479 \mathrm{~nm}$ LED constant.

It may however work out that the light produced in the morning and evening is accepted to be dimmer than in the middle of the day anyway, enabling this kind of set up to mimic the desired intensity output. Tests measuring the range in lumens are necessary to answer that question. To negate the intensity variation all together a light source would need to be produced which decreased the overall output of each LED to the point where both LED types were on (somewhere near $3100 \mathrm{~K}$ ). This means that the only time each LED type was at maximum intensity would be at the extremes (2500 and $7500 \mathrm{~K}$ ). Every 
other Kelvin value would require both LED types to operate at reduced amperage so that combined luminescence would not exceed what a single type of LED would emit at maximum current. While this would solve the intensity variation issue it may not be practical for illumination applications to operate the LEDs at such a reduced current.

\section{Recommendations}

Based upon the results thus far, the most prominent method to replicate the Kelvin scale between 2500 and 7500 would be to approximate it in a linear manner. As discussed previously, in order to change the color values over a linear range two color sources must be adjusted independently. Based upon the defined Kelvin values in this study, daylight is most likely found to be between 2500 and 5500 Kelvin. Therefore it would be better to make the initial color at 2500 Kelvin instead of 7500 Kelvin because the light source will spend most of its time closer to the 2500 Kelvin value as opposed to the 7500 Kelvin value. With a light source producing approximately 2500 Kelvin, a second light source producing $484 \mathrm{~nm}$ light is required to change the chromaticity values along the linear Kelvin scale approximation trend (Figure 57).

The apparatus needed to display this range of Kelvin values may consist of an array of 2500 Kelvin LEDs and $484 \mathrm{~nm}$ blue LEDs (Figure 73). 


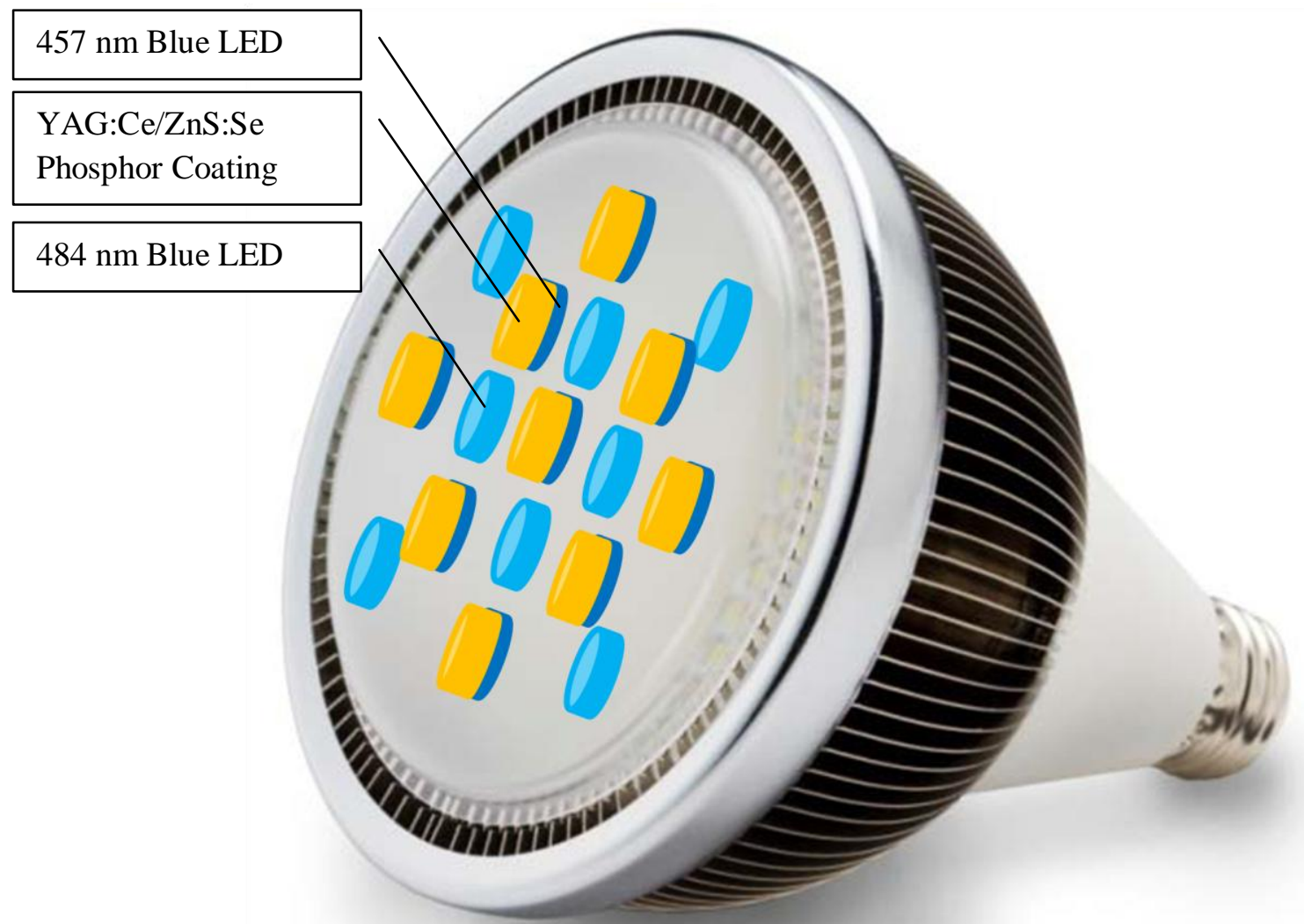

Figure 73 - Cartoon depiction of a LED lamp with $457 \mathrm{~nm}$ blue LEDs coated with a YAG:Ce and ZnS:Se phosphor combination dispersed periodically along with $484 \mathrm{~nm}$ blue LEDs.

The 2500 Kelvin LEDs could be manufactured by applying a specific ratio of YAG:Ce and $\mathrm{ZnS}:$ Se over a blue LED (457 $\mathrm{nm}$ peak in this study). At full intensity to the 2500 Kelvin LEDs and zero power to the blue LEDs the light source will illuminate with a 2500 Kelvin value. That color would then change along the linear Kelvin approximation curve as the blue LEDs are turned on and increased to maximum intensity. Depending upon the concentration of 2500 Kelvin LEDs and blue LEDs the point where they are each at maximum intensity may likely be around 3500 Kelvin. The intensity of the 2500 
Kelvin LEDs can then be decreased while holding the $484 \mathrm{~nm}$ blue LEDs at a maximum intensity until the desired Kelvin value (between 3500 and 7500 Kelvin) is obtained. Again, It is recommended to use a $484 \mathrm{~nm}$ blue LED as opposed to blue phosphor excited by UV LEDs due to the drastic changes in intensity required throughout the 2500 to 7500 Kelvin scale. 


\section{CHAPTER IX: RECOMMENDATIONS FOR FUTURE WORK}

\section{Addressing Efficiency Issues with Phosphors Separate from the LED Die}

Methods of increasing the distance of phosphor to the LED die may likely benefit from a set up which reduces the dispersion of light from the LED die prior to interaction with the phosphor substrate. Greater entrance angles into the phosphor substrate may promote backscattering which increases the quantity of light being directed back onto the LED die consequently increasing the die temperature. A possible solution to this method may be to cast a waveguide between the LED die and the phosphor substrate (Figure 74).

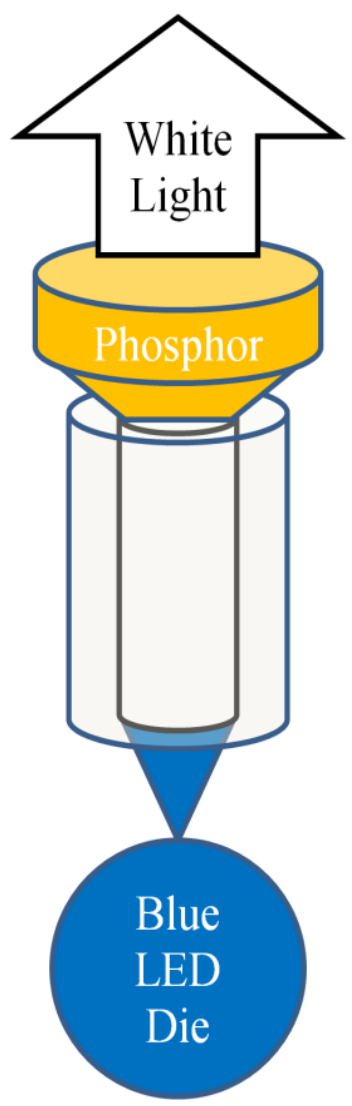

Figure 74 - In this kind of apparatus a waveguide would need to be deposited directly over the LED. Light would be directed with minimal loss or dispersion through the waveguide and in the phosphor substrate. 
This would enable the directionality of the LED light to be maintained while moving the phosphor further away. A waveguide could be manufactured with two slightly different versions of PDMS that would have different indexes of refraction.

\section{Thin Layer Gaussian Profile}

In an attempt to develop a white light pixel for applications which pertain to General LED, a method of developing a Gaussian layer in PDMS was theorized. This method mimics grayscale lithography by exposing negative photoresist in eight separate instances of various dimensions. The purpose of mimicking grayscale lithography is to produce a Gaussian-like profile within a $200 \mu \mathrm{m}$ thick PDMS layer. A mask was created to enable two different methods of achieving this structure (Figure 75). One method involves exposing each structure with light of the same integration time. The other method requires integration times which differ depending upon the layer being exposed. The first method (outermost pixels) contains structures in the mask which contain dimensions based on the separations that correspond to equal changes in amplitude within the Gaussian profile. The second method (innermost pixels) contains equal changes in distances between sequential structures. 


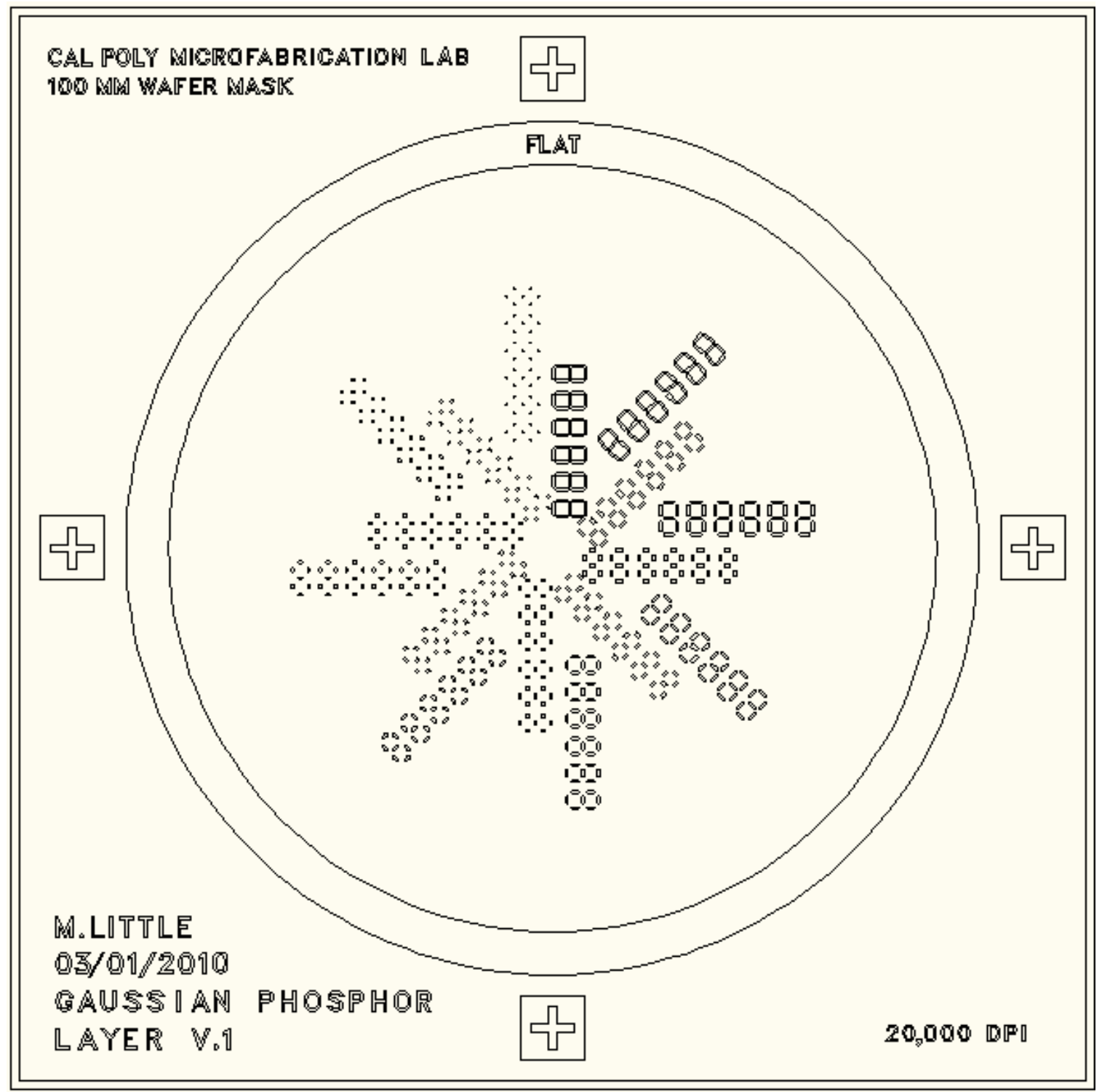

Figure 75 - The mask was designed based on size and spacing of numeric oriented pixels produced by General LED for various applications. The final six digit configuration is designed to overlay on top of the LED pixels to change blue LEDs into an apparent uniform white color.

A Gaussian-like profile may be obtained by rotating the mask in $45^{\circ}$ increments clockwise prior to each exposure starting with the smallest dimension structures on the mask. This will enable the smallest regions to be exposed for the longest amount of time resulting in the deepest penetration into the negative photoresist. The result will be 
Gaussian shaped divots for each pixel in the pattern of six sequential digital number eight figures. A mixture of phosphor in PDMS may then be spin coated on the hardened negative photoresist and cured. When the phosphor/PDMS layer is peeled away from the negative photoresist it may be placed above the blue LED pixels (flat side down) to provide a uniform color of white light (Figure 76).

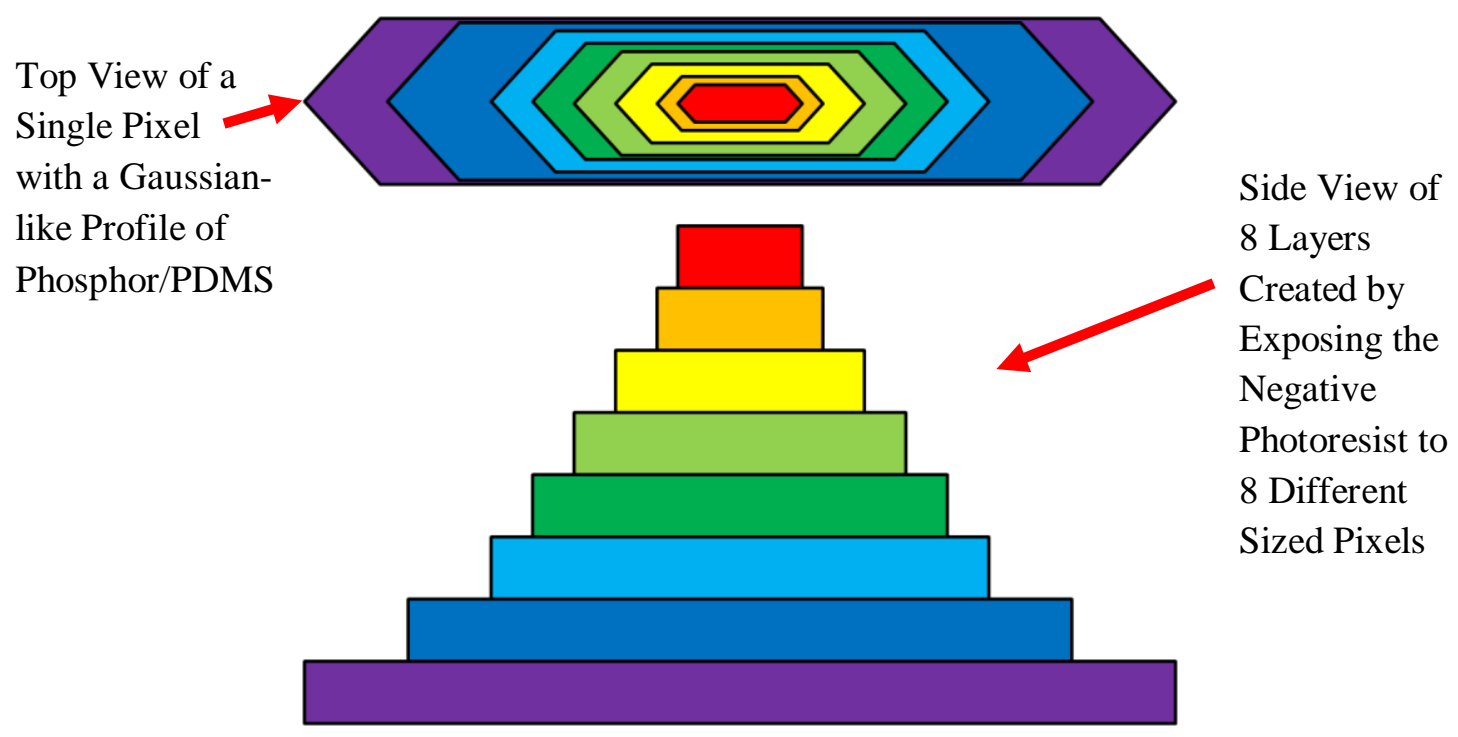

Figure 76 - Top and side view of a Gaussian-like profile created above each of the seven pixels which comprise a single digital number eight.

\section{Phosphor Characterization}

X-ray fluorescence (XRF) revealed what was originally believed to be BAM:Eu phosphor, based on the photoluminescent properties, is actually a zinc-based phosphor. More analysis is necessary to determine the exact phosphor elemental make-up and unfortunately this discovery came at the completion of this report. It may be useful to study not only the elemental composition of each of the phosphor but also characterize their size distribution and dispersion throughout the PDMS substrates in a manner related to photoluminescent efficiency. 


\section{CHAPTER X: GLOSSARY}

1931 CIE $2^{\circ}$ Standard Observer: A two dimensional diagram used to provide a numeric correlation to color values. The intensity of the color is neglected meaning variations of gray are equivalent to white light in this color space. This particular diagram was developed in 1931 by the International Commission on Illumination (CIE).

BAM:Eu: A compilation of barium, magnesium, and aluminum with trace amounts of europium to form a crystalline structure. The particular combination of elements is used in phosphor form throughout this thesis.

Circadian Rhythm: An approximately 24-hour cycle in living entities based on the environment with one of the major influences being daylights cycle.

Correlated Color Temperature (CCT): The appearance of a theoretical black body radiator as described in SI unit of absolute temperature (Kelvin). CCT temperatures indicate the color appearance of a white light source.

Color Point: A single point on a chromaticity diagram described with an $\mathrm{x}$ and $\mathrm{y}$ coordinate.

Color Rendering Index (CRI): A measurement with scale from 0 to 100 depicting a light sources ability to illuminate an object as compared to a black body radiator (such as sunlight) for a given Kelvin temperature. The test is typically conducted by measuring the chromaticity value deviation of eight colored samples illuminated with a light source as compared to a black body radiator source.

Crystal Field Theory: A model which describes electronic properties in transition metals. The model properly explains splitting of electron energy levels often observed in their orbitals.

Electromagnetic Spectrum: A continuous range of electromagnetic radiation energies typically characterized by wavelengths, frequencies, or energies. The spectrum ranges from Gamma rays to visible light to radio waves.

Electronic Energy Transition: The transition of an electron from an excited state to a ground state without giving off energy in the form of phonons.

Exciton: An electron-hole pair with no net charge and typically found in insulators or semiconductors. While this particle is imaginary it is useful for indicating the existence of an excited electron and a corresponding hole. 
Gaussian Distribution: A continuous normal distribution often referred to as a bell curve. The statistical function is symmetrical about a mean value at its peak.

High-Powered LEDs: Typically considered an LED which may be driven at hundreds of milliamps to more than an ampere. The lumen output is much greater than typical miniature LEDs which operate near 10 milliamps.

Light Emitting Diode (LED): A solid state device comprised of multiple layers of semiconducting, conducting, and insulating materials which create isolated electrodes sandwiching a pn-junction. Under forward bias electrons recombine with holes to produce photons in a process known as electroluminescence.

Luminescence: Light produced from an object at low temperatures and therefore not by black body radiation as incandescence is.

Luminous Efficacy: Expressed in lumens per watt (lumens/W). A measure of the quantity of lumens emitted from a source divided by the consumed wattage.

Luminous Emittance: A term to describe the amount of emitted light from a surface of a given area. Reported in lux (lumens $/ \mathrm{m}^{2}$ )

Phosphor: A combination of materials which are capable of emitting photons when excited by electrons or photons. They typically consist of a host lattice with trace amounts of an impurity atom to provide luminescent centers.

Phosphor-Converted LED (pcLED): An LED with a phosphor layer positioned between its die and an observer. The color of light is different from the plain LED as some of the emitted wavelengths undergo photoluminescence.

Photoluminescence: A luminescent process where photons are absorbed and re-radiated after a given time period with a different wavelength.

Polydimethylsiloxane (PDMS): The most widely used silicon-based organic polymer. It may be found in shampoos, lubricating oils, and contact lenses.

Vibronic Energy Transition: A combination of electronic and vibrational transitions which an excited electron may take on its way to a ground state.

Vibrational Energy Transition: Strictly a transition in energy an electron may go through which transmits phonons to or from a lattice.

YAG:Ce: Throughout this report YAG:Ce is considered a phosphor comprised of yttrium, aluminum, oxygen, and trace amounts of cerium. 
ZnS:Se: A semiconductor based phosphor with a Wurtzite crystal structure made from zinc and sulfur and trace amounts of selenium distributed throughout. 


\section{CHAPTER XI: WORKS CITED}

1) "Light-Emitting Diode: Efficiency and Operational Parameters." 2006. Wikipedia. 13 October $2008<\mathrm{http}: / /$ en.wikipedia.org/wiki/Light-emitting_diode>.

2) "Lumileds: LED Lighting Solutions." 2008. Philips. 13 October 2008 <http://www.philipslumileds.com/solutions/>.

3) Pang, Siew It. "Phosphor Converted LED with Improved Uniformity and Having Lower Phosphor Requirements." Patentdocs (13 Oct. 2008).

4) "Color Rendering Index (CRI) Explained." Full Spectrum Solutions. 15 August 2009 $<$ http://www.fullspectrumsolutions.com/cri_explained.htm>.

5) "LED Technical: Lighting Terms Reference and Glossary." Bravolight. 24 April 2010 <http://www.bravolight.com/LED-Technical.html>.

6) Keiner, Louis E. "Media-Lab Archive." 2007. Simon Fraser University. 24 April 2010 $<$ http://www.sfu.ca/medialab/archive/2007/387/Resources/Images/EM\%20Spectrum/ElectromagneticSpectrum.png>.

7) Sutter, Rober T and Michael W. Davidson. "Light Emitting Diodes." 15 June 2006. Molecular Expressions. 2 December 2008 <http://micro.magnet.fsu.edu/primer/java/leds/basicoperation/>.

8) Emsley, John. The Shocking History of Phosphorus. London: Macmillan, 2000.

9) Ronda, Cees and Alok Srivastava. "Luminescence Science and Display Materials." Electrochemical Society (Spring 2006): 55-57.

10) Barron, Andrew R and Carissa Smith. "Crystal Structure." 28 January 2010. Connexions. 15 April $2010<$ http://cnx.org/content/m16927/latest/>.

11) Xie, Rong-Jun and Naoto Hirosaki. "Silicon-Based Oxynitride and Nitride Phosphorss for White LEDs - A Review." Science and Technology of Advanced Materials (27 August 2007): 588-600.

12) Pan, Yuexiao, Mingmei Wu and Qiang Su. "Tailored Photoluminescence of YAG:Ce Phosphor Through Various Methods." Journal of Physics and Chemistry of Solids (2004): 845-850.

13) Shionoya, Shigeo. Phosphor Handbook: VI: Phosphors for Cathode Ray Tubes. Florida: Boca Raton, 1999. 
14) Bigelow, Ron. "Perception: Color \& Luminosity - Part II." RonBigelow. 15 April 2010 $<$ http://www.ronbigelow.com/articles/color-perception-2/perception-2.htm>.

15) Brown, Ronald F. Solid State Physics: An Introduction for Scientists and Engineers. San Luis Obispo: El Corral Bookstore, 2006.

16) "Color Rendering Index (CRI) Explained." Full Spectrum Solutions. 15 August 2009 $<$ http://www.fullspectrumsolutions.com/cri_explained.htm>.

17) Narendran, Nadarajah. "Improved Performance White LED." Fifth International Conference on Solid State Lighting, Proceedings of SPIE. Bellingham, WA: International Society of Optical Engineers, 20005. 45-50.

18) "The 2nd Generation: Thin Layer Solar Cells." NODE: Nederlands Onderzoeksplatform Duurzame Energievoorziening. 11 February $2010<$ http://www.energyresearch.nl/energyoptions/solar-cells/background/types/thin-layers/>.

19) Callister, William D. Jr. Materials Science and Engineering an Introduction: 6th Edition. New York: John Wiley \& Sons, 2003.

20) Tipler, Paul A and Ralph A. Llewellyn. Modern Physics: 3rd Edition. New York: W. H. Freeman, 1999.

21) Kasap, Safo O. Principles of Electronic Materials and Devices: 3rd Edition. New York: McGraw-Hill Companies, Inc, 2006.

22) Costello, Ken. "Nature of Light and a Modern View of the Atom." 19 April 2008. ChemistryLand. 2 May $2010<$ http://www.chemistryland.com/CHM130W/10ModernAtom/Spectra/ModernAtom.html>.

23) Freiberg, Arvi. "Exciton Self-Trapping in Bacterial Antennas Promotes Light Harvesting Efficiency." Fuusika Instituut: Tartu Ulikool. 15 April 2010 <http://www.fi.tartu.ee/biannual0001/freiberg.htm>.

24) Blasse, G and B. C. Grabmaier. Luminescent Materials. Emeryville: Springer-Verlag Telos, 1994.

25) "BYW01A/PTCW01AN Phosphor Datasheet." Phosphortech. 1 December 2009 $<$ https://secure40.securewebsession.com/hisham.site.aplus.net/data_sheets/BYW01A.pdf $>$.

26) "Polydimethylsiloxane." 7 May 2010. Wikipedia. 9 May 2010 <http://en.wikipedia.org/wiki/Polydimethylsiloxane>. 
27) "PDMS Process Notes." 12 September 2006. Stanford: Biomimetics \& Dexterous Manipulation Laboratory. 20 October 2008 <http://bdml.stanford.edu/twiki/bin/view/Rise/PDMSProceSS>.

28) "Garnet." 29 April 2010. Wikipedia. 21 May 2010 <http://en.wikipedia.org/wiki/Garnet>.

29) Kuklja, Maija M and Ravindra Pandey. "Atomistic Modeling of Native Point Defects in Yttrium Aluminum Garnet Crystals." Journal of the American Ceramic Society (1999): 2881-2886.

30) "BUVR03/PTCR03 Phosphor Datasheet." Phosphortech. 1 December 2009 <https://secure40.securewebsession.com/hisham.site.aplus.net/data_sheets/BUVR03.pdf>

31) "Zinc Sulfide." 22 November 2009. Wikipedia. 2 December 2009 <http://en.wikipedia.org/wiki/Zinc_sulfide>.

32) "Polydimethylsiloxane (PDMS)." Silicones Science On-Line. 1 December 2009 $<$ http://www.silicones-science.com/chemistry_pdms.html>.

33) "Sylgard 184 Silicone Elastomer Kit (Curing Agent)." Dow Corning Corporation MSDS. 17 December 2008.

34) "Information about Dow Corning Brand Silicone Encapsulants." Dow Corning Corporation Product Information (2008).

35) Ishihara, Kazuhiko. "Phosphorylcholine Group-Immobilized Surface Prepared on PDMS Membrane by In Situ Reaction for Its Reduced Biofouling." Journal of NanoBioTechnology (2007): 83-88.

36) Roth, Leandro E. "Bulk Hydrosilylation Reaction of PDMS Chains Catalyzed by a Platinum Salt: Effect of the Initial Concentrations of Reactive Groups on the Final Extent of Reaction." Journal of Polymer Science (2003): 1099-1106.

37) Chung, Dae-Won. "Study on the Effect of Platinum Catalyst for the Synthesis of PDMS Grafted with Polyoxyethylene." Journal of Industrial Engineering Chemistry (2007): 571577.

38) Keller, Michael W. "A Self-Healing PDMS Elastomer." Advanced Functional Materials (2007): 2399-2404.

39) Venkataraman, S. K. "Finite Shear Strain Behavior of a Crosslinking PDMS Near its Gel Point." Rheologica Acta (1990): 423-432.

40) Chambon, Francois. "Stopping of Crosslinking Reaction in a PDMS Polymer at the Gel Point." Polymer Bulletin (1985): 499-503. 
41) Dunleavy, Dr. John. "Sulfur as a Catalyst Poison." Platinum Metals (2006): 110.

42) Fischer, T. E. "Model Experiments on the Poisoning of Platinum Catalysts by Sulfur." Journal of Catalysts (1978): 24-34.

43) Kim, Y and S. Kang. "Investigation of the Degradation Mechanisms in BaMgA110O17:Eu2+ Phosphor: On the Influence of Thermal Process on Operational Durability." Applied Physics (2010): 429-434.

44) "UVB03 Phosphor Datasheet." Phosphortech. 19 October 2009.

45) Becker, William G and Allen Bard. "Photoluminescence and Photoinduced Oxygen Adsorption of Colloidal Zinc Sulfide Dispersions." Journal of Physical Chemistry (1983): 4888-4893.

46) Wu, Y.L and A.I.Y Tok. "Surface Modification of ZnO Nanocrystals." Applied Surface Science (2007): 5473-5479.

47) Oosthuizen, L and H.C. Swart. "ZnS:Cu,Al,Au Phosphor Degradation Under Electron Excitation." Applied Surface Science (1997): 9-14.

48) Anpo, Masakazu and Yutaka Kubokawa. "Photoluminescence of Zinc Oxide Powder as a Probe of Electron-Hole Surface Processes." Journal of Physical Chemistry (1984): 55565560 .

49) Bard, Allen J. "Photoelectrochemistry and Heterogeneous Photocatalysis at Semiconductors." Journal of Photochemistry (1979): 59-76. 


\section{Appendix A: Method of Production (Pouring, Spin Coating, and Plasma Bonding)}

\section{Method of Production - Pouring}

The following procedure was designed to provide a consistent method of creating phosphor and PDMS substrates. This is the simplest method but is limited to a minimum thickness of approximately $1 \mathrm{~mm}$. The first step is to gather the materials. A disposable mixing cup and a stir rod are necessary for initiating the reaction. Slygard 184 Silicone Elastomer Base can be dispensed with a common plastic $12 \mathrm{~mL}$ syringe. Slygard 184 Silicone Elastomer Curing Agent is best dispensed with a common plastic $3 \mathrm{~mL}$ syringe. Phosphor and a suitable size, large enough to cut out the desired end structure, petri dish are also needed. The last piece of equipment is a scale which can read to a minimum of hundredths of a gram to insure process repeatability.

When using a $90 \mathrm{~mm}$ petri dish the following procedure will yield a $2.5 \mathrm{~mm}$ thick phosphor/PDMS sample. All PDMS substrates have been produced using a base to agent ratio of 10 to 1 respectively. Begin by measuring the weight of the mixing cup. Add 20 $\mathrm{mL}$ of base and $2 \mathrm{~mL}$ of agent. Mix ingredients rapidly for 3 minutes while attempting to create as much air pockets as possible. Measure the weight of the cup and PDMS to determine the total amount of PDMS in the cup. Add the specified amount of phosphor knowing the desired weight percentage and the PDMS weight. Mix the phosphor using the same method as before. Place the mixing cup into a vacuum chamber and apply the maximum amount of vacuum which doesn't allow the mixture to overflow the cup. At this point weigh the petri dish and add $16.32 \mathrm{~g}$ of phosphor/PDMS. To minimize air 
pockets during the pour, create a steady flow over the middle of the petri dish which travels the shortest possible distance between the edge of the cup and the top of the mixture in the petri dish. Bake the sample at $60^{\circ} \mathrm{C}$ for one hour to cure.

\section{Method of Production - Spin Coating}

For sub-1mm thick phosphor/PDMS substrates a method of spin coating has been utilized. The specific steps in this procedure yield a layer with an approximate thickness of $80 \mu \mathrm{m}$. Follow all of the steps stated for the pouring method until the step of pouring into the petri dish. At that point a wafer needs to be prepared which will provide a uniform flat surface for the spin coating process.

Dip a silicon wafer into Piranha (solution with 10 parts sulfuric acid and 1 part hydrogen sulfide), at $70^{\circ} \mathrm{C}$, for 10 minutes to remove organics. Rinse the wafer off with deionized (DI) water and dip into buffered oxide etch (BOE) 3 short times to remove the oxide layer. Again, follow this step with a DI water rinse. Remove any liquid with low-purity nitrogen $\left(\mathrm{N}_{2}\right)$ and place upon a hot plate, at $100^{\circ} \mathrm{C}$, for 15 seconds. Then place the wafer on a room temperature aluminum base for 30 seconds to allow for a cool down. In a petri dish, lay a paper towel down and add 5 drops of Trichloroperfluorooctylsilane. Place the wafer on top of the saturated towel and close the lid. A monolayer will form on the silicon and prevent PDMS from bonding to it in future steps. Remove the wafer after 30 seconds. Be sure to throw waste into the acid container.

Create an aluminum foil shielding on the inside wall of the spin coater and secure with tape. Insure vacuum is applied to the chuck, then center the wafer onto the chuck. Pour 
the PDMS, with the same technique stated in the previous section, until $75 \%$ of the wafer is covered. Select program "T" and verify the parameters include a 20 second spin at 400 rpm followed by a 30 second spin at $1000 \mathrm{rpm}$. Run program, remove wafer, and cure at a $60^{\circ} \mathrm{C}$ temperature for one hour.

Depending upon application it may be necessary to adhere the thin phosphor/PDMS substrate to a thicker PDMS substrate for the purpose of removing it from the wafer and handling thereafter. In that instance use the pouring method to produce a pure PDMS substrate. If using a $120 \mathrm{~mm}$ petri dish, use $40 \mathrm{~g}$ of PDMS to obtain a $2 \mathrm{~mm}$ thickness.

To remove the thin layer of phosphor/PDMS from the wafer and prevent tearing the surface needs to be plasma treated and adhered to a stronger substance. Two options are available in Cal Poly's cleanroom, the reactive ion etcher (RIE) or the atmospheric plasma gun.

\section{Plasma Bonding}

The RIE should be operated according to the following procedure. Open all valves leading to the RIE from the low-purity nitrogen, the high-purity oxygen and sulfur hexafluoride. Turn on the Rohwedder and check the RIE power box to insure all lights are on. Turn PLC on, insure DC bias voltage is approximately 0 (under 10), and the PS to RF generator should be on. Change the pressure regulator valve to open and turn MP to on which turns on the vacuum pump. Turn the RUF on (opens the vacuum valve) and wait till the equipment is pumped down to 50 mTorr. At that point turn the RUF off and turn the vent on which opens the purge valve to let nitrogen flow in. Wait for the top to 
pop open then place the wafer and the pure PDMS sample in the chamber with the side to be treated facing up. Turn the vent off, the RUF on, and again wait till it's pumped down to 50 mTorr. Turn on gas 2 (oxygen) and note the minimum pressure. Switch butterfly to remote, equalize to the pressure set point (50 mTorr), and insure the DC bias voltage never goes over 500. Turn RF to on and use the adjusting knob to expose the samples with 20 watts for 3 seconds. Turn off the process gas then vacuum the chamber. When the pressure reaches 300 mTorr vent and repeat the vacuum process (removes the plasma byproducts). Vent the chamber, remove the samples, turn RF to on, and pump down.

The plasma gun technique may produce less uniform bonding results as opposed to the RIE outcome. Begin by opening the argon valve and powering on the equipment. Do not adjust the plasma intensity or fine adjustment knobs. Position the gun about a half inch from the surface to be treated. Depress the "Ready" button and steadily move the gun over the entire surface once in a motion which takes approximately 10 seconds. Depress the yellow "Plasma" button when finished.

Following either the RIE or plasma gun treatment, the pure PDMS needs to be carefully placed onto the thin phosphor/PDMS coated wafer to minimize air pockets. Then bake the two adhered materials at $60^{\circ} \mathrm{C}$ for one hour to anneal the plasma treated surfaces. The thin phosphor/PDMS layer may then be removed from the wafer by carefully removing the thick pure PDMS taking the desired phosphor/PDMS layer with it. 


\section{Appendix B: Apparatus Set Up}

The method to collect data was held constant for every test involving the concentration, thickness, and distance variables. Begin by turning on the power to the LED, the Linear Motion Controller, the Spectrometer, the DAQ, and the computer. Initialize the Avantes Software and select "Start" to begin collecting data. Then auto-integrate the spectrum to obtain an unsaturated image on the screen. The next step is to center the LED below the fiber optic cable. To complete this task first insure that the motors are turned off as indicated on the LCD screen of the Linear Motion Controller. Second, manually adjust the two horizontal motors until the maximum blue wavelength peak appears on the Avantes software. The spectrum may become saturated during this procedure. When that occurs simply auto-integrate the spectrum again. Place the black cover over the entire assembly to minimize external lighting influences and select auto-integrate one last time. Be sure to reset each motors position to 0.000 using controls on the Linear Motion Controller. Then select Save Spectrum and choose the appropriate folder. Turn the LED off and select "Save Dark Spectrum." Under setup select "Subtract save dark" and then select options under the same menu. Click external trigger settings, select the lowest bullet on the menu, check the box, and click OK. "External Trigger Mode: Hardware" should appear on the screen which indicates the DAQ can control the timing to save spectrums. Turn the LED back on and open the program titled "LED Mapping." Select Play and controls for the linear stage motors will appear. Enter in the range in millimeters desired to examine as well as the increment in millimeters. Then select "Start Mapping." Spectrums will save to the file specified when "Save Spectrum" is selected. 


\section{Appendix C: Data Processing Using Excel Macros}

Processing the large quantity of data outputted after each test was completed using Microsoft Excel. A blank Excel document titled "Blank Mapping Template" can be used to create the intensity and chromaticity matrices. Upon opening the Excel document, enable Macros and select "Design Mode."

\section{Import Data}

On the first tab, "Import from .trt," double click on "Load Files" to bring up the code. The second line of code says "PATH $=\ldots \ldots \ldots$. .." Insert the entire directory for the location of the '.trt' files which were produced following the testing. Be sure that a 'I' is at the end of the directory location. Exit design mode and click "Load Files." Allow a few hours to import all the files (time depends on the quantity of files and processor speed). When the program is finished select "Check For Missing Data" to insure there were no discontinuities while importing the data.

\section{Intensity Matrices}

Select the "Reorder Raw Files" tab and click "Load Raw Data." This step organizes the data according to position and wavelength. Next select the "Intensity Matrix" tab and enter values under "Map Width/Height" and "Scan Increment." The Map Width/Height value is the overall distance in millimeters scanned from one edge to the adjacent one (i.e. for a range from $-15 \mathrm{~mm}$ to $15 \mathrm{~mm}$ enter 30 ). Matrices at this stage can be directly imported into MatLab for producing 3D images. The next tab, "Intensity Cross-Section," displays the intensities at given wavelength spreads across the entire substrate at $\mathrm{y}=0$. 


\section{Chromaticity Matrices}

To produce chromaticity matrices select the "Chromaticity Conversion" tab and click "Find $\mathrm{x} \& \mathrm{y}$ " to begin calculations. This process creates the $\mathrm{x}$ and $\mathrm{y}$ coordinates for color calculation at each measurement location. When the program is finished select the "Chromaticity Matrix" tab followed by the "Build Matrix" button to build chromaticity matrices. These matrices may also be imported into MatLab for 3D image production. Click the "Chromaticity Cross-Section" tab to display a cross section of chromaticity values. 


\section{Appendix D: Phosphor Datasheet (1/2)}

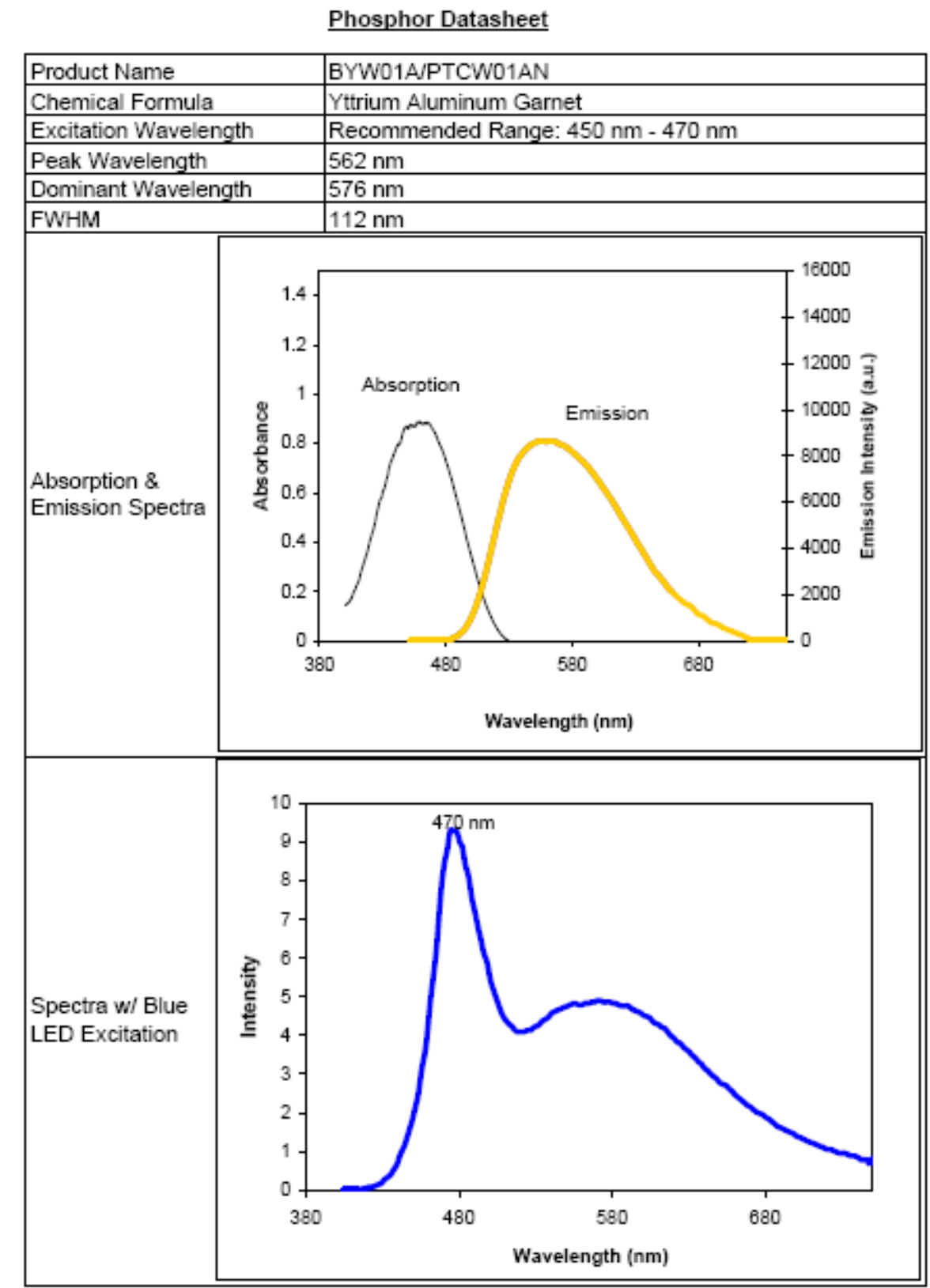

PHOSPHORTECH CORPORATION

WWW.PHOSPHORTECH.COM

Figure 77 - Phosphor datasheet of the specific YAG:Ce phosphor purchased from PhosphorTech. Absorption and Emission profiles are utilized to design a white LED, shown in the profile directly underneath. 


\section{Appendix E: Phosphor Datasheet (2/2)}

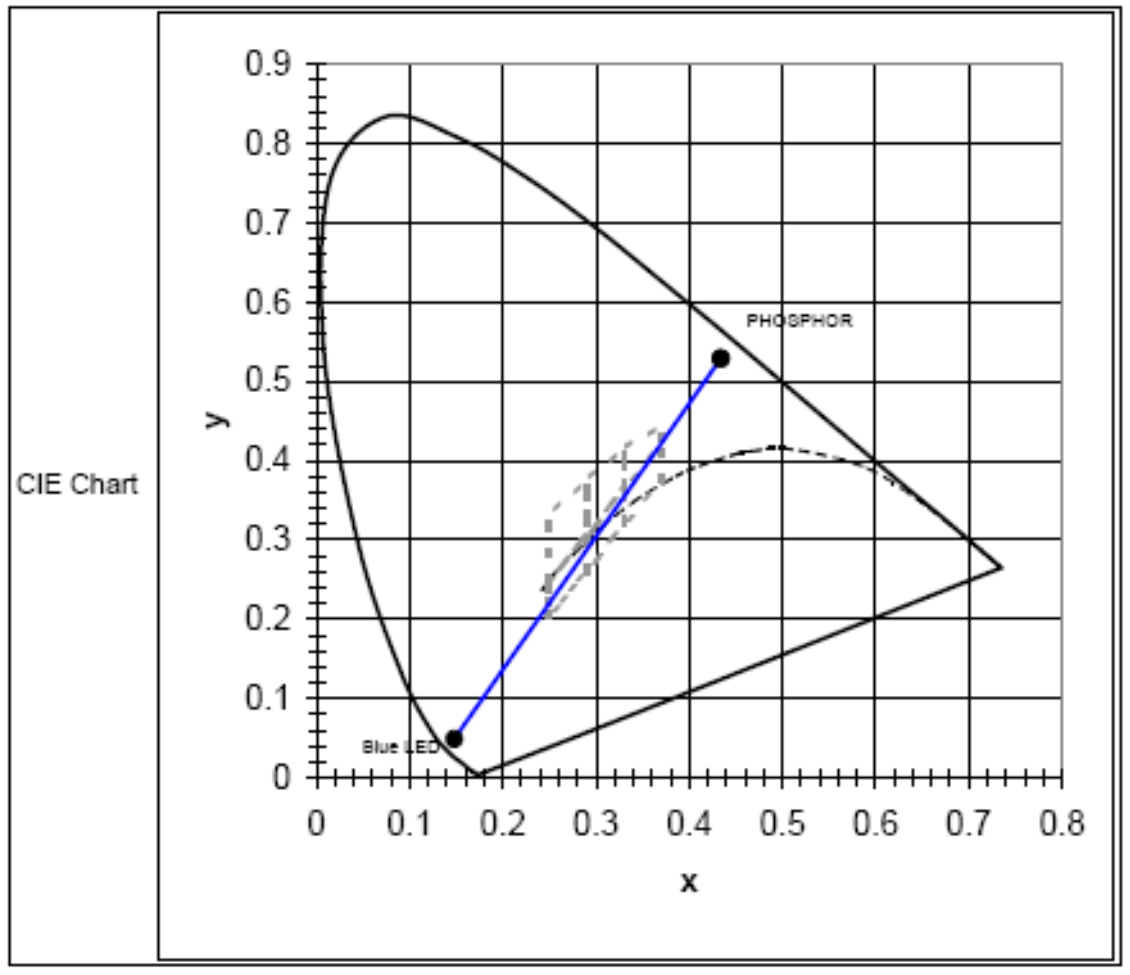

\begin{tabular}{|l|l|}
\hline CIE Coordinates & $(x=0.434, y=0.529) \quad($ CIE 1931) \\
\hline Particle Size & Mean $\sim 9 \mu \mathrm{m}$ \\
\hline Body Color & Yellow \\
\hline Handling Guidelines & See MSDS \\
\hline
\end{tabular}

Figure 78 - All the possible color values obtainable with a given blue LED using the specific YAG:Ce phosphor utilized throughout all of the experiments. 


\section{Appendix F: Predominantly Blue Intensity Profile of Various Concentration Substrates}

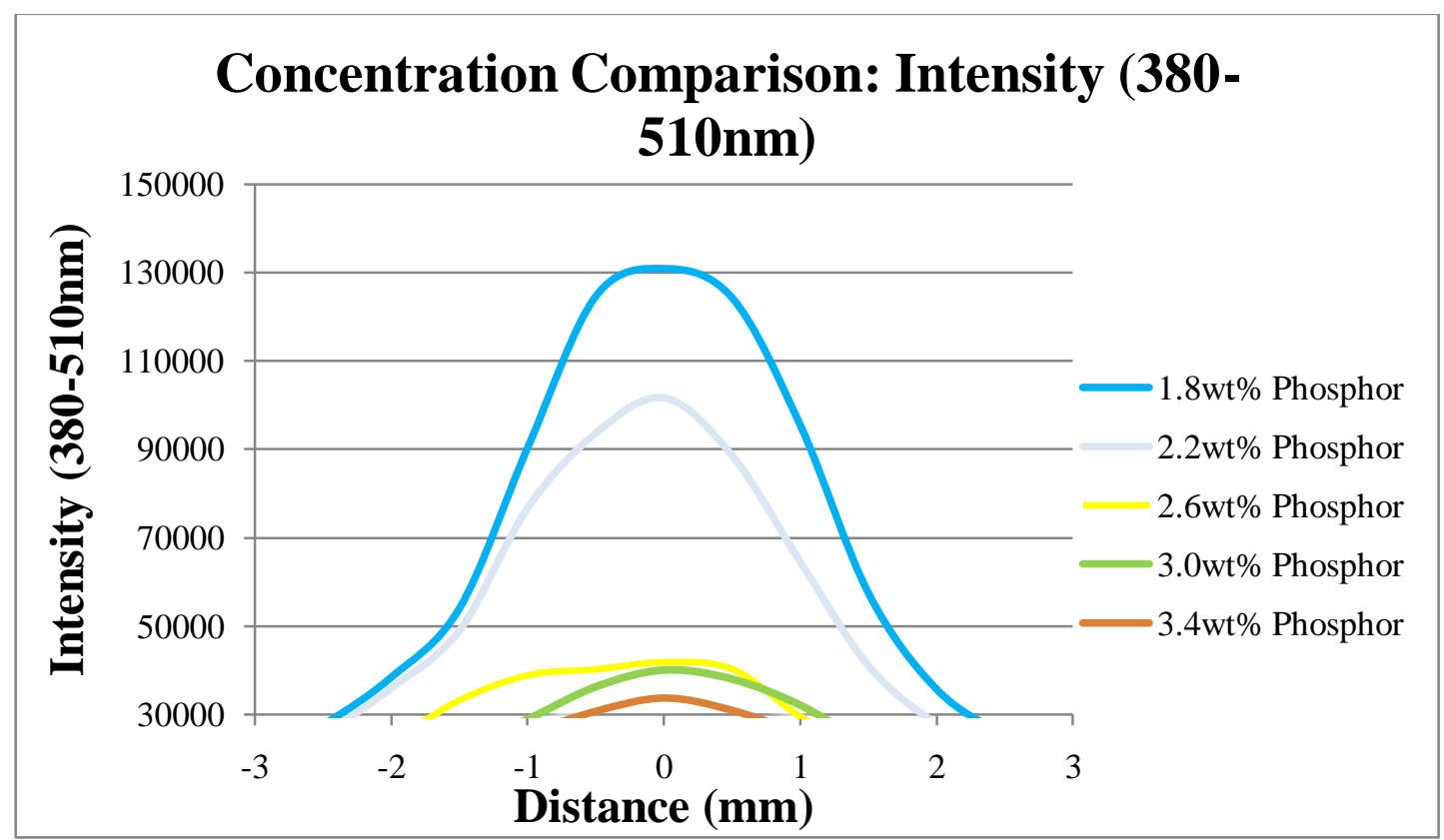

Figure 79 - Intensity comparisons between the five different, $2.5 \mathrm{~mm}$ thick, phosphor wt $\%$ samples. Increasing the amount of phosphor above the LED decreases blue light intensity emitted orthogonal the substrate surface. Intensity profiles here reflect the amount of blue light passing through the substrate. 


\section{Appendix G: Predominantly Yellow Intensity Profile of Various Concentration Substrates}

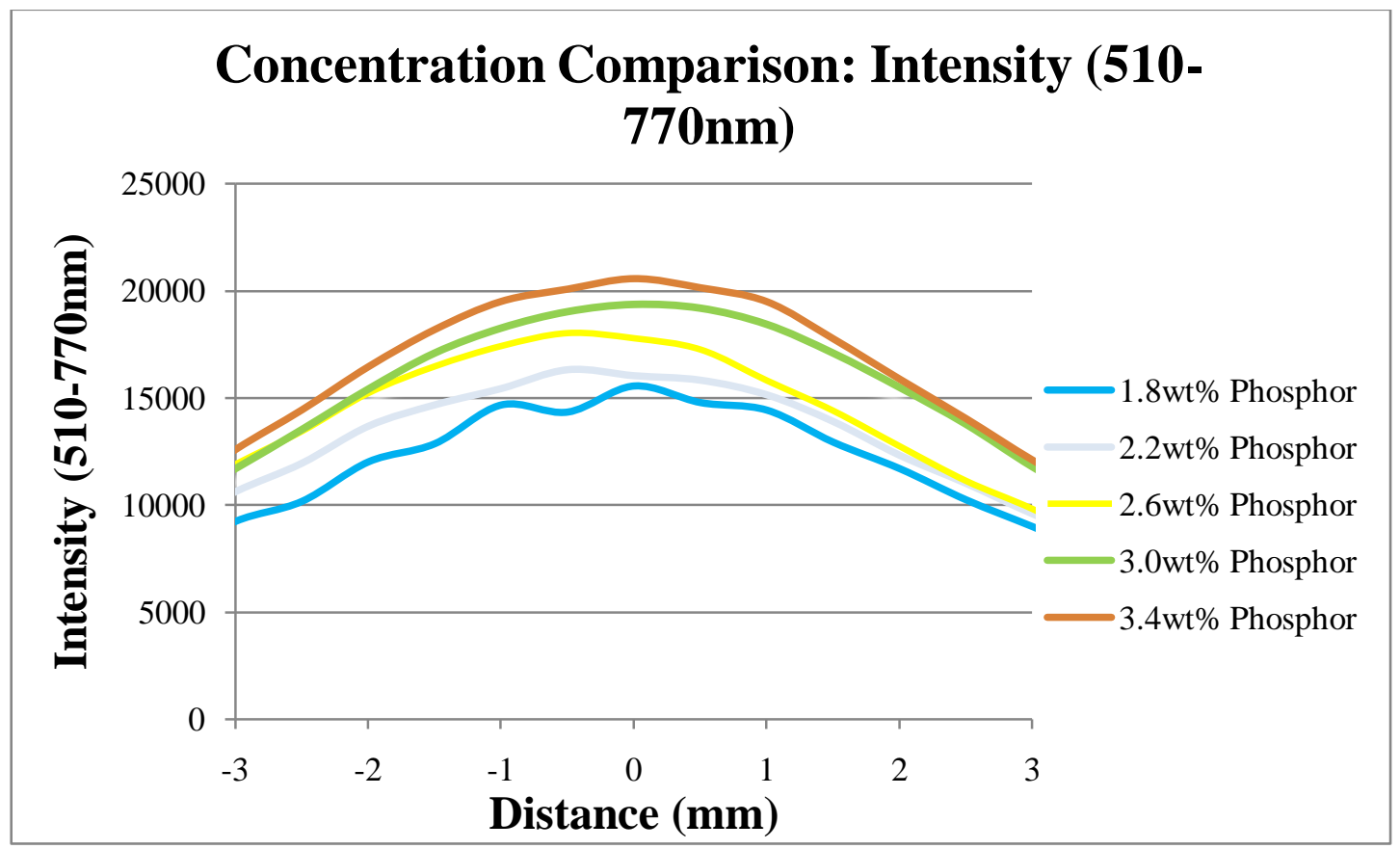

Figure 80 - Intensity comparisons between the five different, $2.5 \mathrm{~mm}$ thick, phosphor wt\% samples. Increasing the amount of phosphor above the LED increases yellow light intensity emitted orthogonal the substrate surface. Intensity profiles here reflect the amount of yellow light emitted by the phosphor. 


\section{Appendix H: Entire Visible Spectrum Intensity Profile of Various Concentration Substrates}

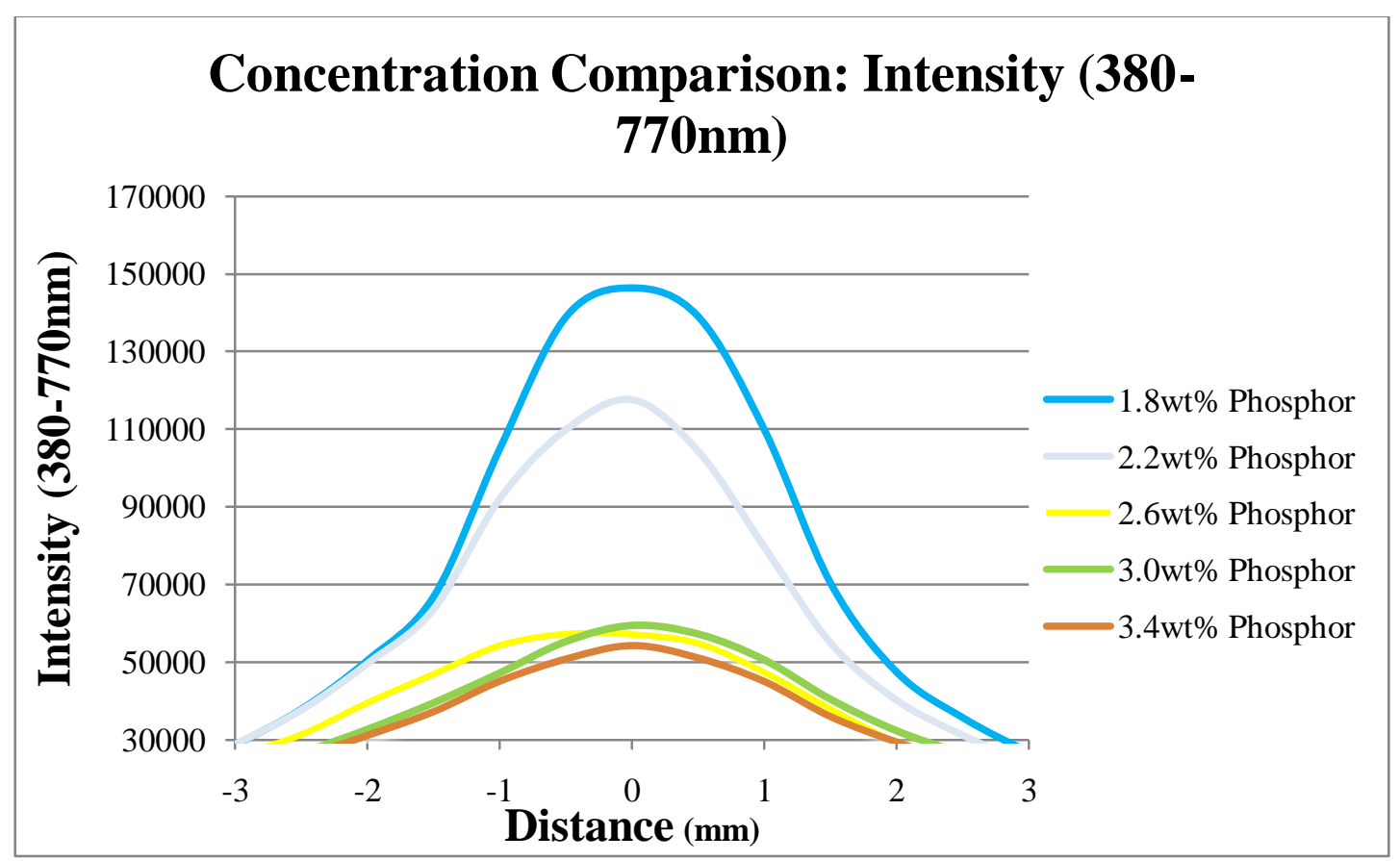

Figure 81 - Intensity comparisons between the five different, $2.5 \mathrm{~mm}$ thick, phosphor wt\% samples. Increasing the amount of phosphor above the LED increases the overall visible light intensity emitted orthogonal the substrate surface. Intensity profiles here reflect the entire visible spectrums intensity. 


\section{Appendix I: X Chromaticity Comparison of Various Substrates Thicknesses}

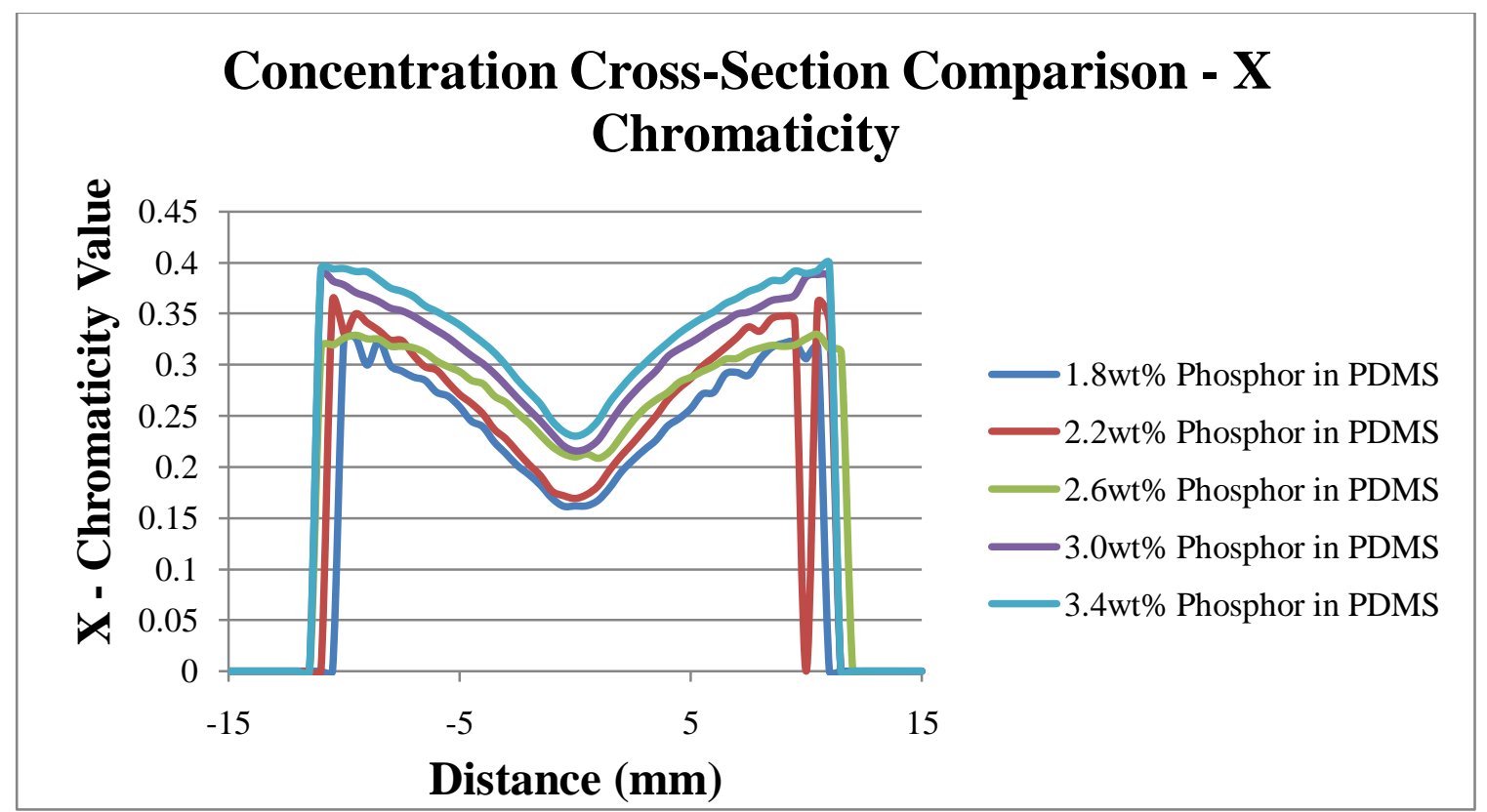

Figure 82 - A cross section of $x$ - chromaticity values indicating the uniformity of color. A minimal change in chromaticity value indicates a more constant color over the cross section of the substrate. Each concentration experiment was conducted using $2.5 \mathrm{~mm}$ thick substrate at a height of $0 \mathrm{~mm}$. 


\section{Appendix J: Y Chromaticity Comparison of Various Substrates Thicknesses}

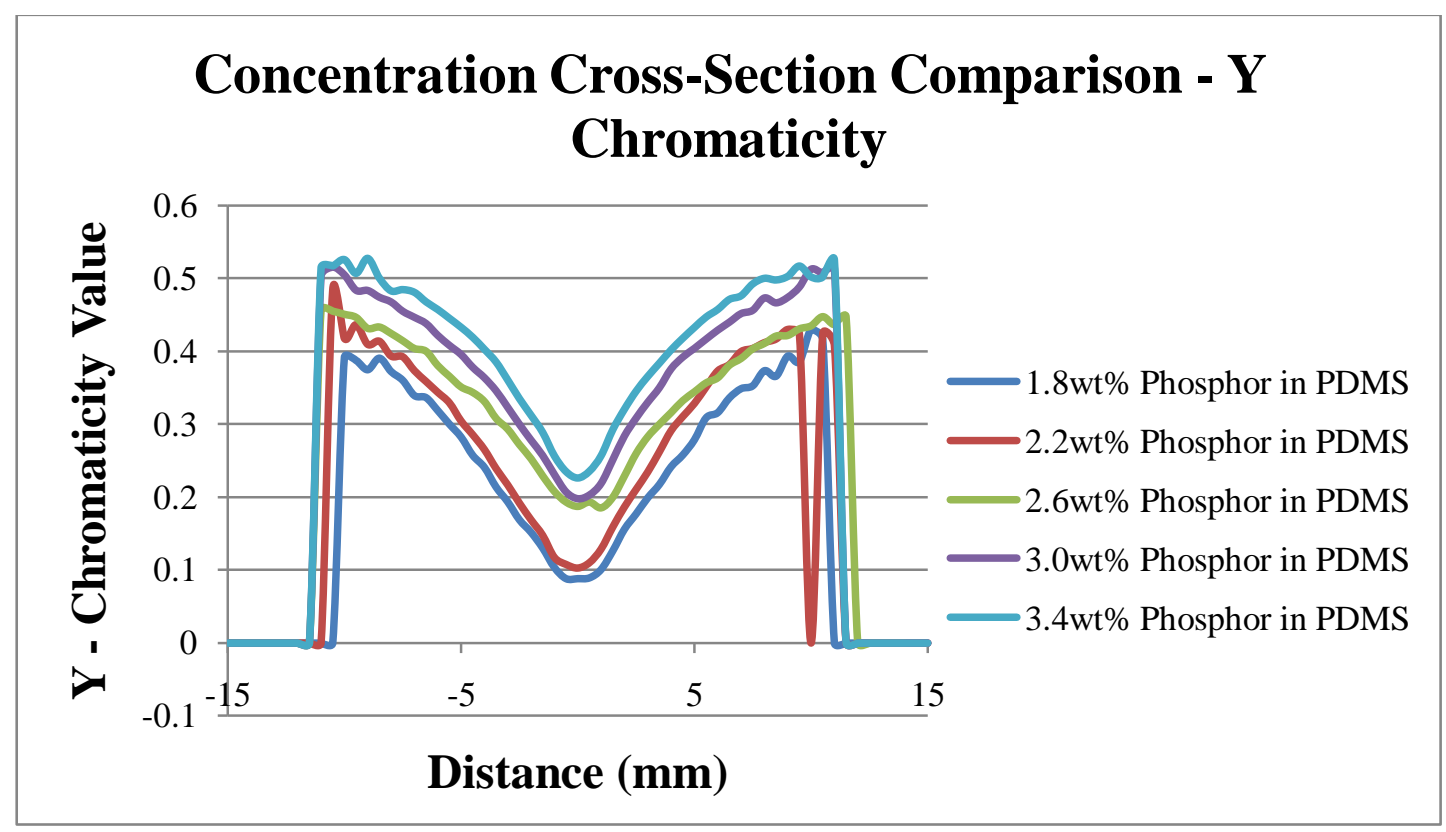

Figure 83 - This graph displays the $y$ - chromaticity values with the same data used to produce Figure 81. Y - chromaticity values are more influenced by changes in phosphor concentration and thickness than $x$ - chromaticity values. Each concentration experiment was conducted using $\mathbf{2 . 5} \mathbf{~ m m}$ thick substrate at a height of $0 \mathbf{~ m m}$. 


\section{Appendix K: X Chromaticity Comparison of Various Height Experiments}

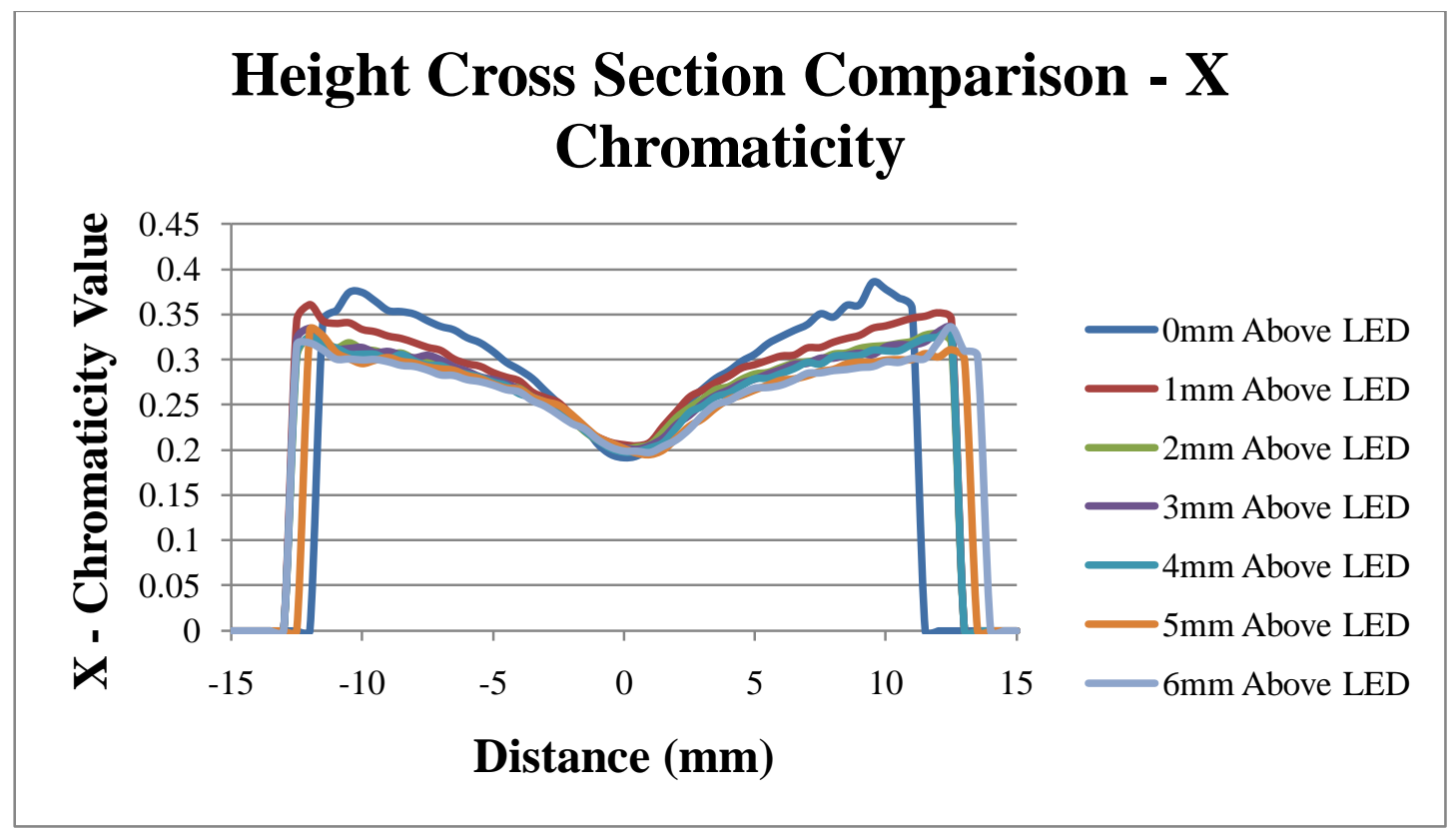

Figure 84 - A cross section of $x$ - chromaticity values indicating the uniformity of color. Adjusting the height has a minimal effect on color values. Each height experiment was conducted using a 1.79 mm thick, $3.8 \mathrm{wt} \%$ substrate. 


\section{Appendix L: Y Chromaticity Comparison of Various Height Experiments}

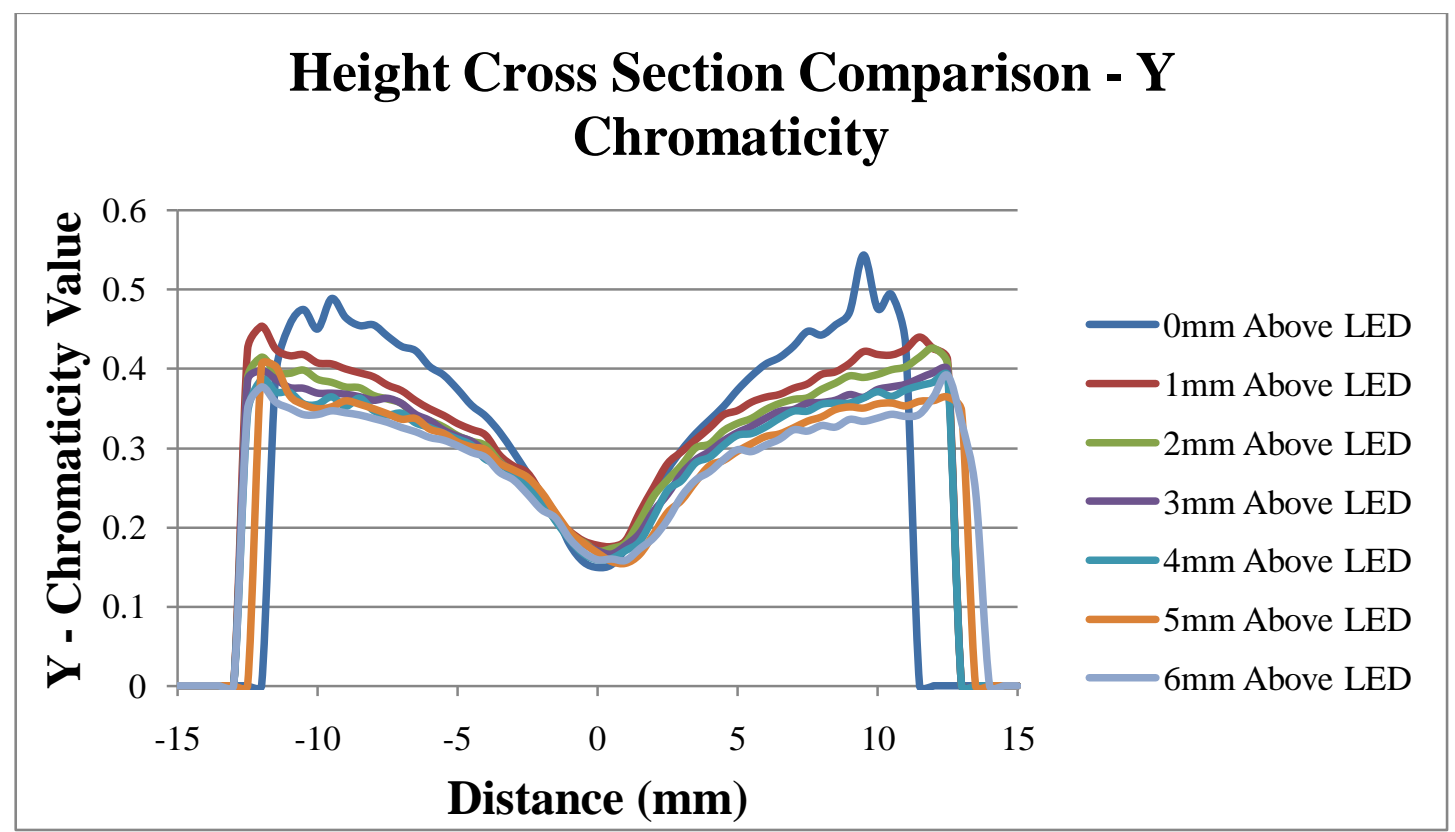

Figure 85 - A cross section of $y$ - chromaticity values indicating the uniformity of color. Adjusting the height has a minimal effect on color values. Each height experiment was conducted using a 1.79 mm thick, $3.8 \mathrm{wt} \%$ substrate. 


\section{Appendix M: X Chromaticity Plot of Thickness versus Concentration Directly Above LED}

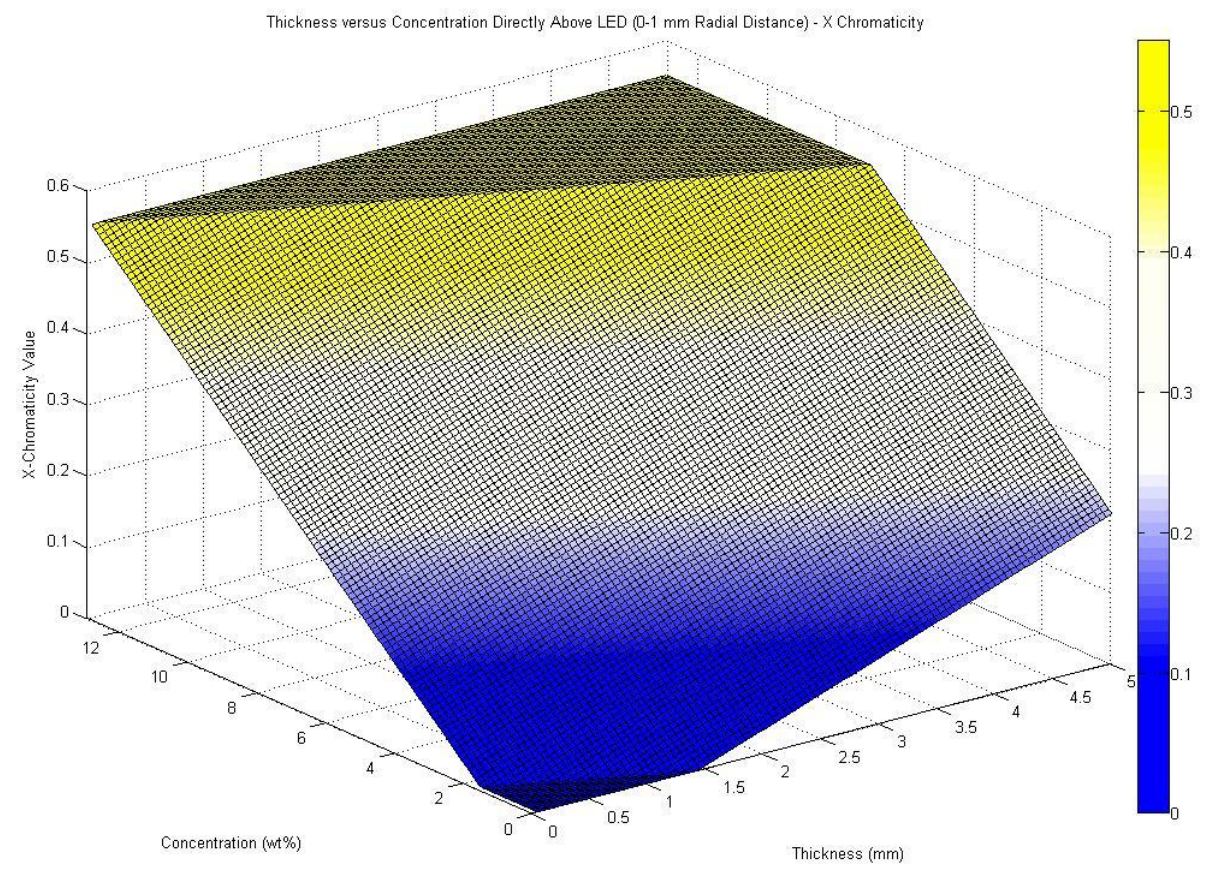

Figure 86 - A 3 dimensional plot color coded to best relay information regarding the actual color of the observed chromaticity values. The plateaus at the bottom and top of the plot are regions which are not valid chromaticity values and therefore are points where the equations fail. This specific plot compares $x$ - chromaticity effects of thickness and concentration in a $1 \mathrm{~mm}$ radial distance from the LED. 


\section{Appendix N: Y Chromaticity Plot of Thickness versus Concentration Directly Above LED}

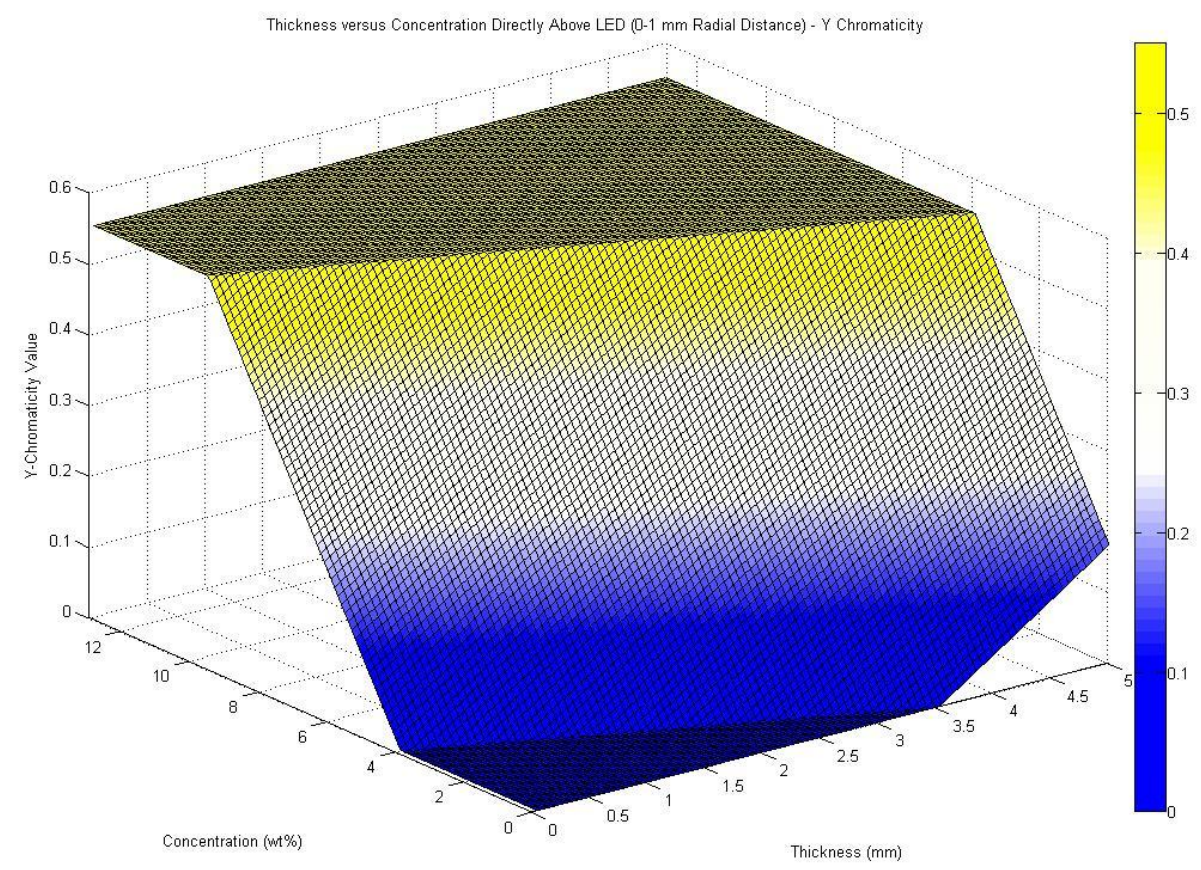

Figure 87 - A 3 dimensional plot color coded to best relay information regarding the actual color of the observed chromaticity values. The plateaus at the bottom and top of the plot are regions which are not valid chromaticity values and therefore are points where the equations fail. This specific plot compares $y$ - chromaticity effects of thickness and concentration in a $1 \mathbf{~ m m}$ radial distance from the LED. 


\section{Appendix O: Y Chromaticity Plot of Thickness versus Concentration Offset Above LED}

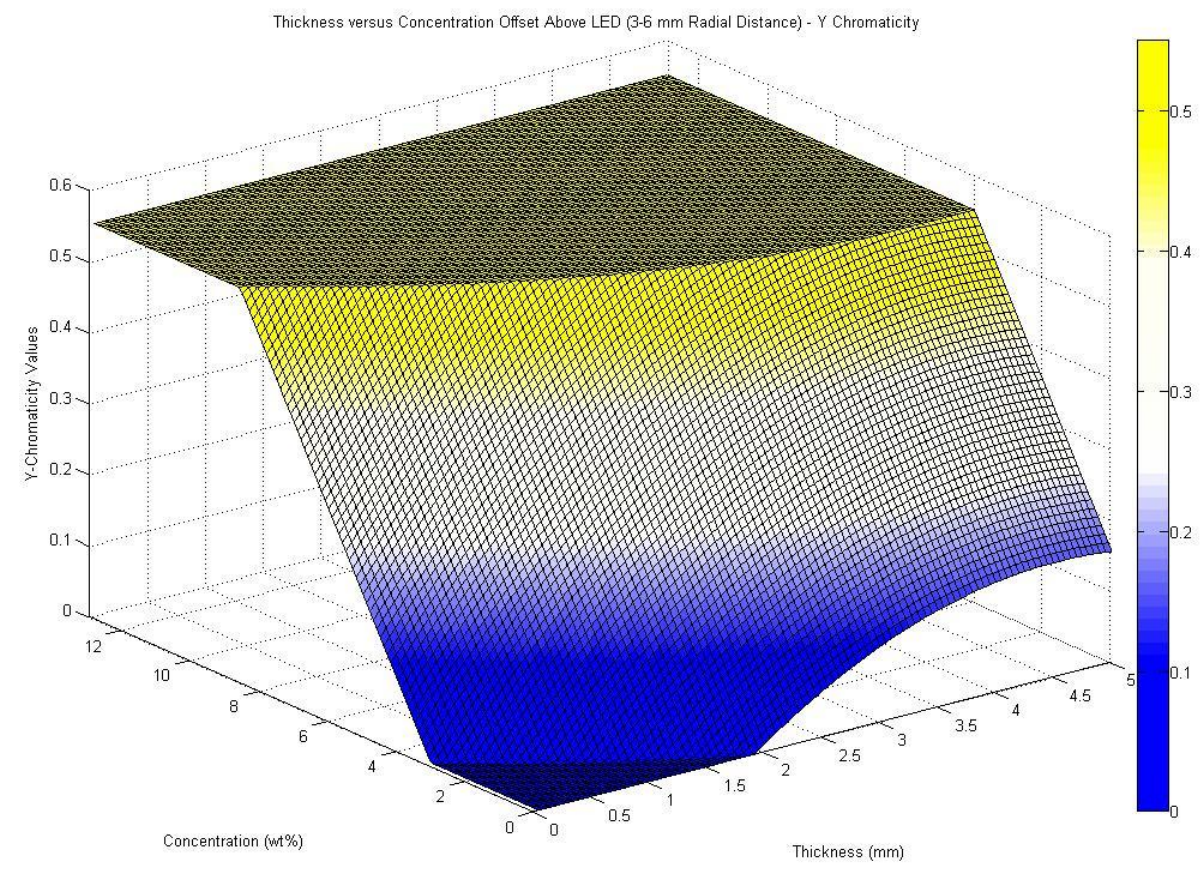

Figure 88 - A 3 dimensional plot color coded to best relay information regarding the actual color of the observed chromaticity values. The plateaus at the bottom and top of the plot are regions which are not valid chromaticity values and therefore are points where the equations fail. This specific plot compares $y$ - chromaticity effects of thickness and concentration over a 3 to $6 \mathrm{~mm}$ radial distance from the LED. 


\section{Appendix P: X Chromaticity Plot of Height versus Thickness Directly Above LED}

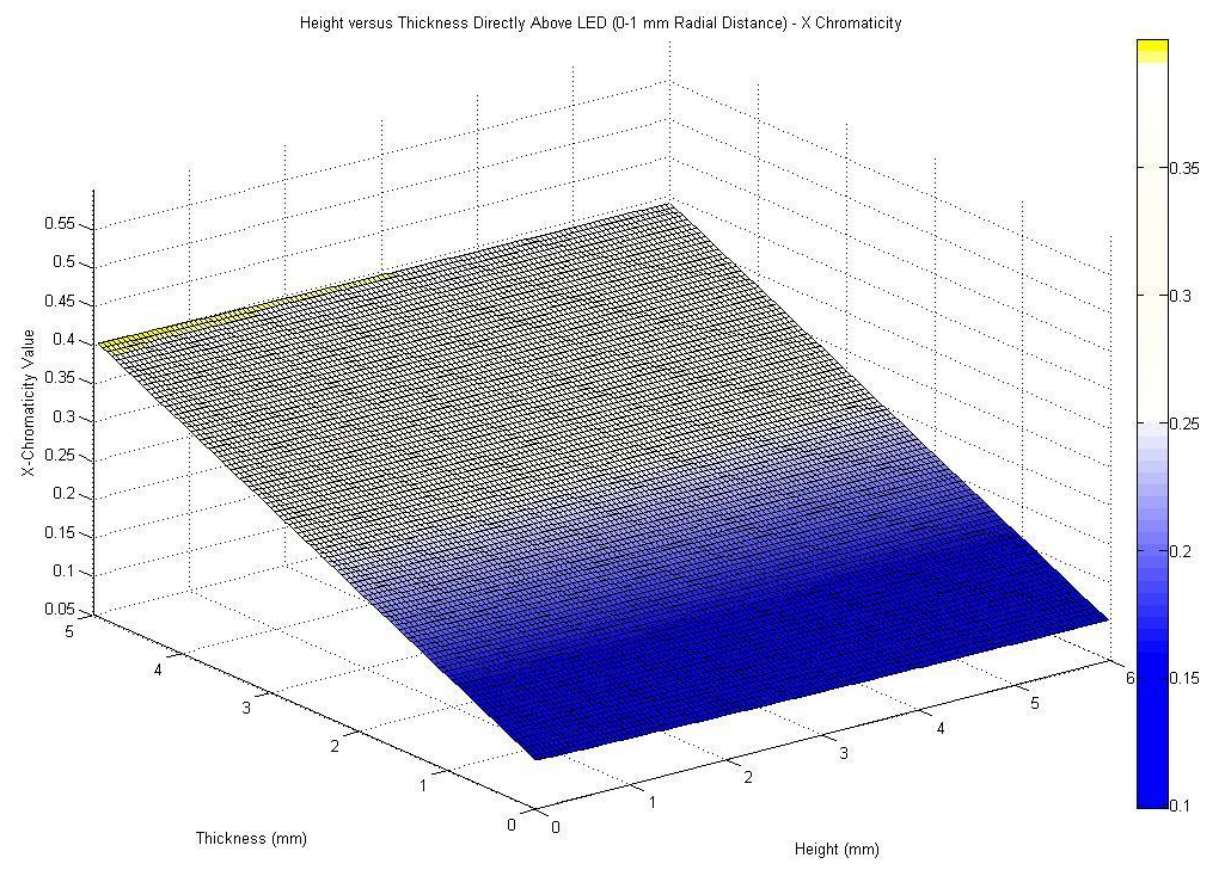

Figure 89 - A 3 dimensional plot color coded to best relay information regarding the actual color of the observed chromaticity values. The plateaus at the bottom and top of the plot are regions which are not valid chromaticity values and therefore are points where the equations fail. This specific plot compares $x$ - chromaticity effects of height and thickness in a $1 \mathrm{~mm}$ radial distance from the LED. 


\section{Appendix Q: Y Chromaticity Plot of Height versus Thickness Directly Above LED}

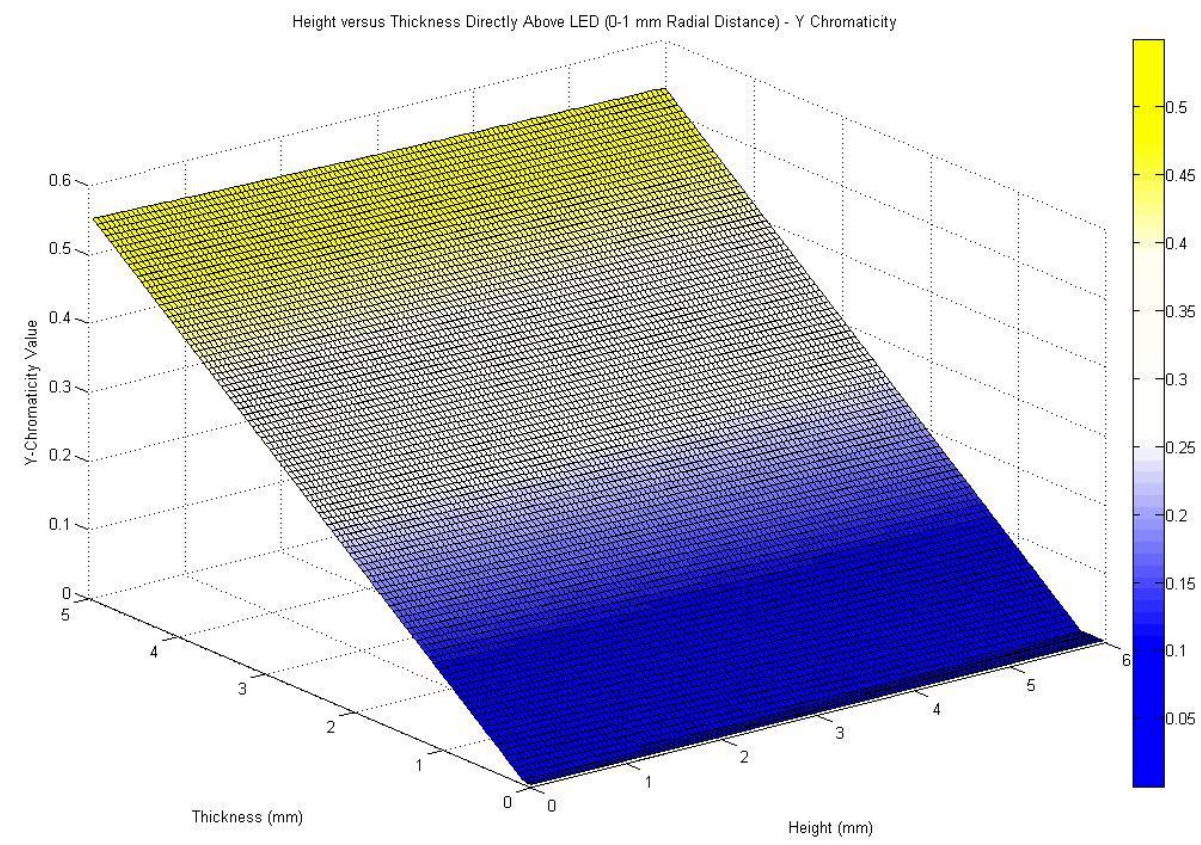

Figure 90 - A 3 dimensional plot color coded to best relay information regarding the actual color of the observed chromaticity values. The plateaus at the bottom and top of the plot are regions which are not valid chromaticity values and therefore are points where the equations fail. This specific plot compares $y$ - chromaticity effects of height and thickness in a $1 \mathrm{~mm}$ radial distance from the LED. 


\section{Appendix R: X Chromaticity Plot of Height versus Thickness Offset Above LED}

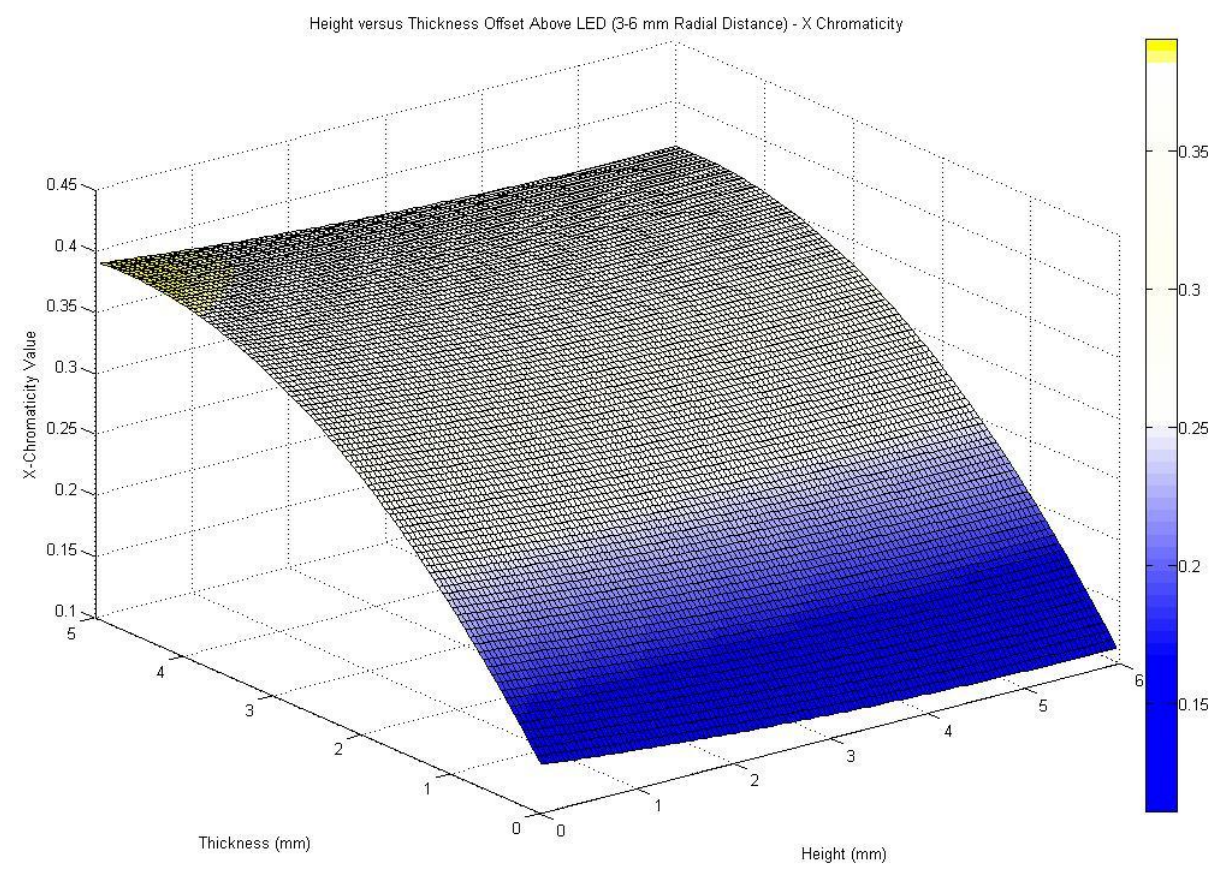

Figure 91 - A 3 dimensional plot color coded to best relay information regarding the actual color of the observed chromaticity values. The plateaus at the bottom and top of the plot are regions which are not valid chromaticity values and therefore are points where the equations fail. This specific plot compares $x$ - chromaticity effects of height and thickness over a 3 to $6 \mathrm{~mm}$ radial distance from the LED. 


\section{Appendix S: Y Chromaticity Plot of Height versus Thickness Offset Above LED}

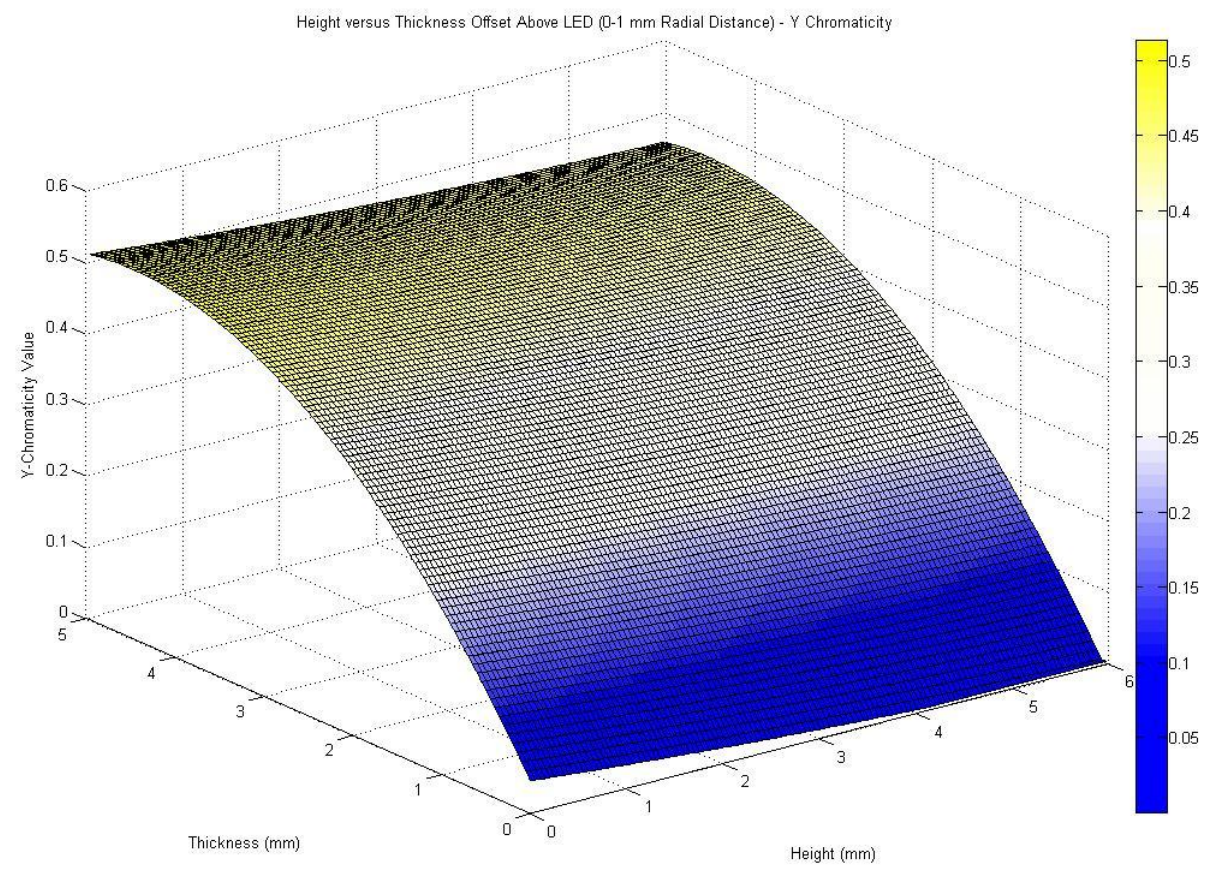

Figure 92 - A 3 dimensional plot color coded to best relay information regarding the actual color of the observed chromaticity values. The plateaus at the bottom and top of the plot are regions which are not valid chromaticity values and therefore are points where the equations fail. This specific plot compares $y$ - chromaticity effects of height and thickness over a 3 to $6 \mathrm{~mm}$ radial distance from the LED. 


\section{Appendix T: X Chromaticity Plot of Height versus Concentration Directly Above LED}

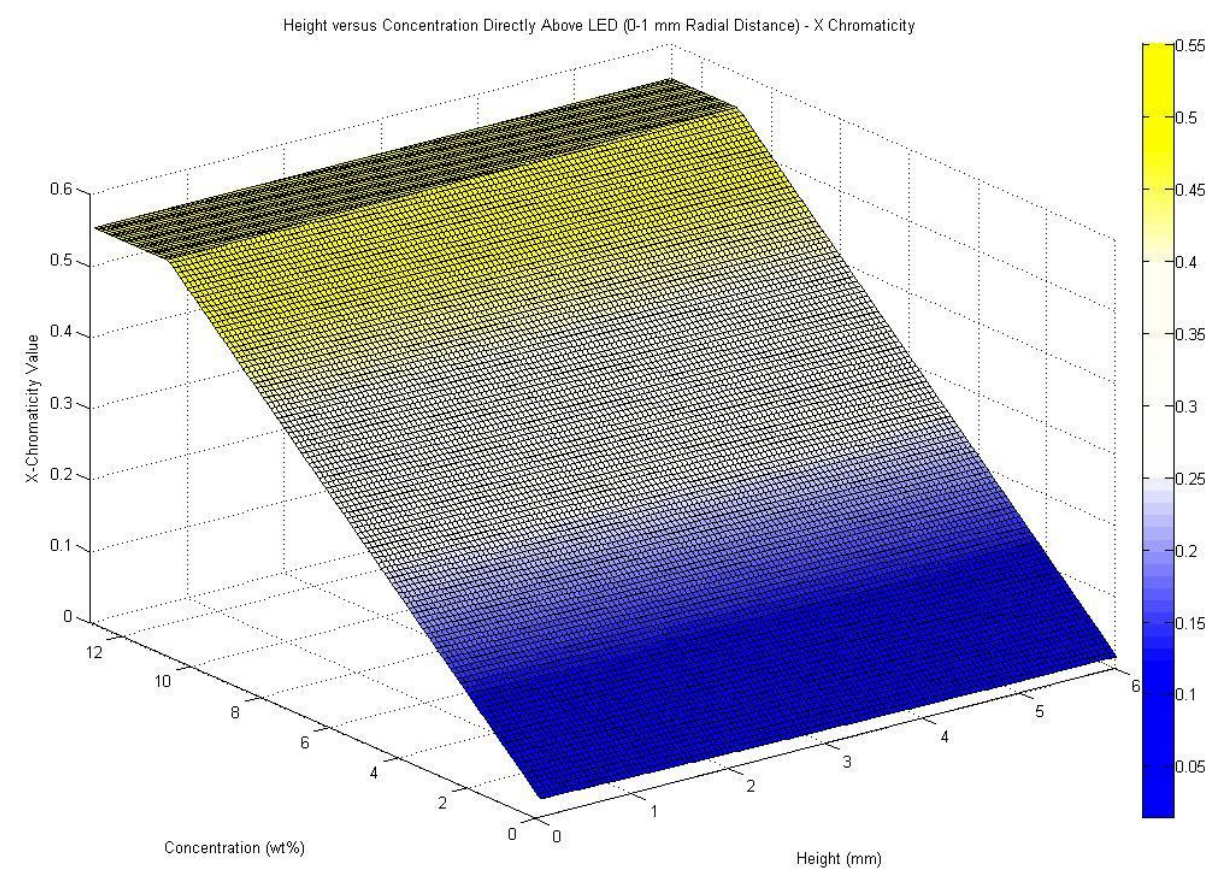

Figure 93 - A 3 dimensional plot color coded to best relay information regarding the actual color of the observed chromaticity values. The plateaus at the bottom and top of the plot are regions which are not valid chromaticity values and therefore are points where the equations fail. This specific plot compares $x$ - chromaticity effects of height and concentration in a $1 \mathrm{~mm}$ radial distance from the LED. 


\section{Appendix U: Y Chromaticity Plot of Height versus Concentration Directly Above LED}

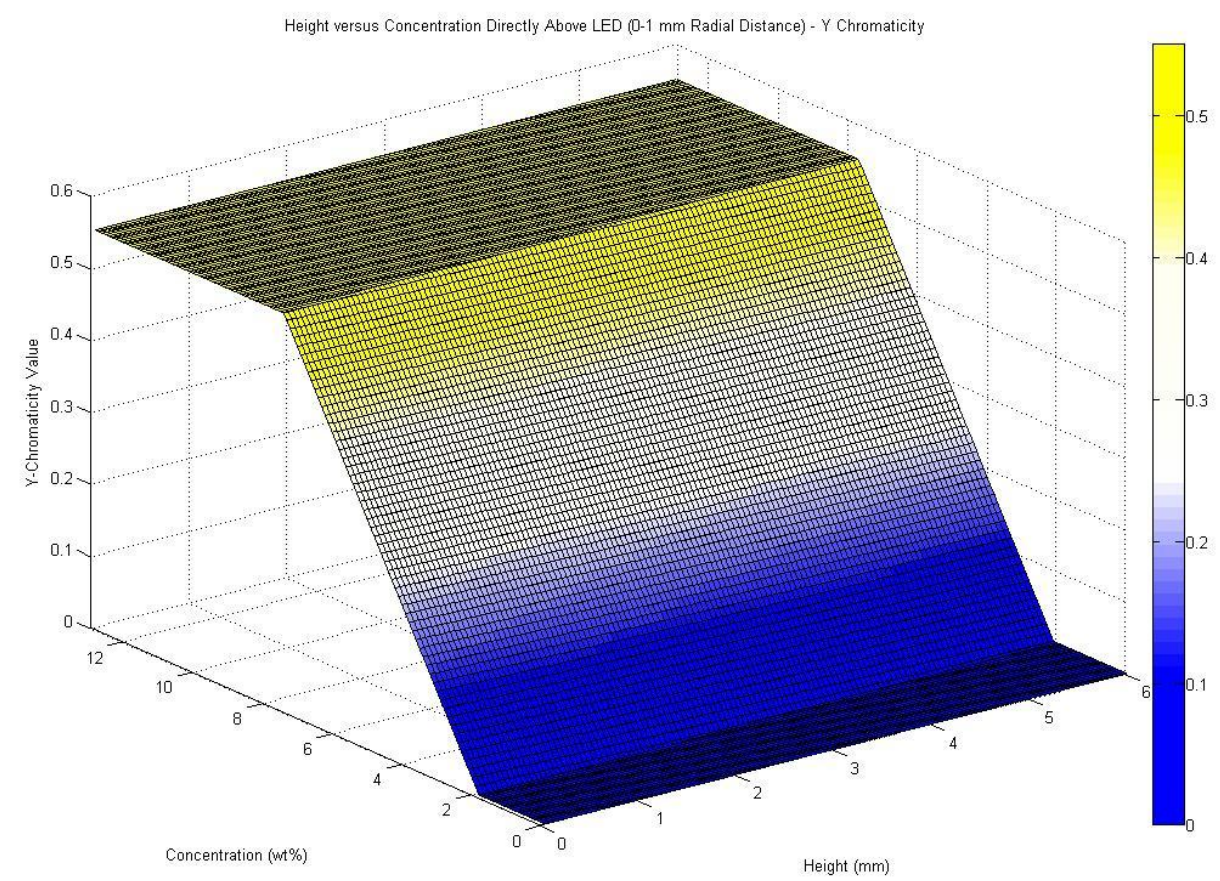

Figure 94 - A 3 dimensional plot color coded to best relay information regarding the actual color of the observed chromaticity values. The plateaus at the bottom and top of the plot are regions which are not valid chromaticity values and therefore are points where the equations fail. This specific plot compares $y$ - chromaticity effects of height and concentration in a $1 \mathrm{~mm}$ radial distance from the LED. 


\section{Appendix V: X Chromaticity Plot of Height versus Concentration Offset Above LED}

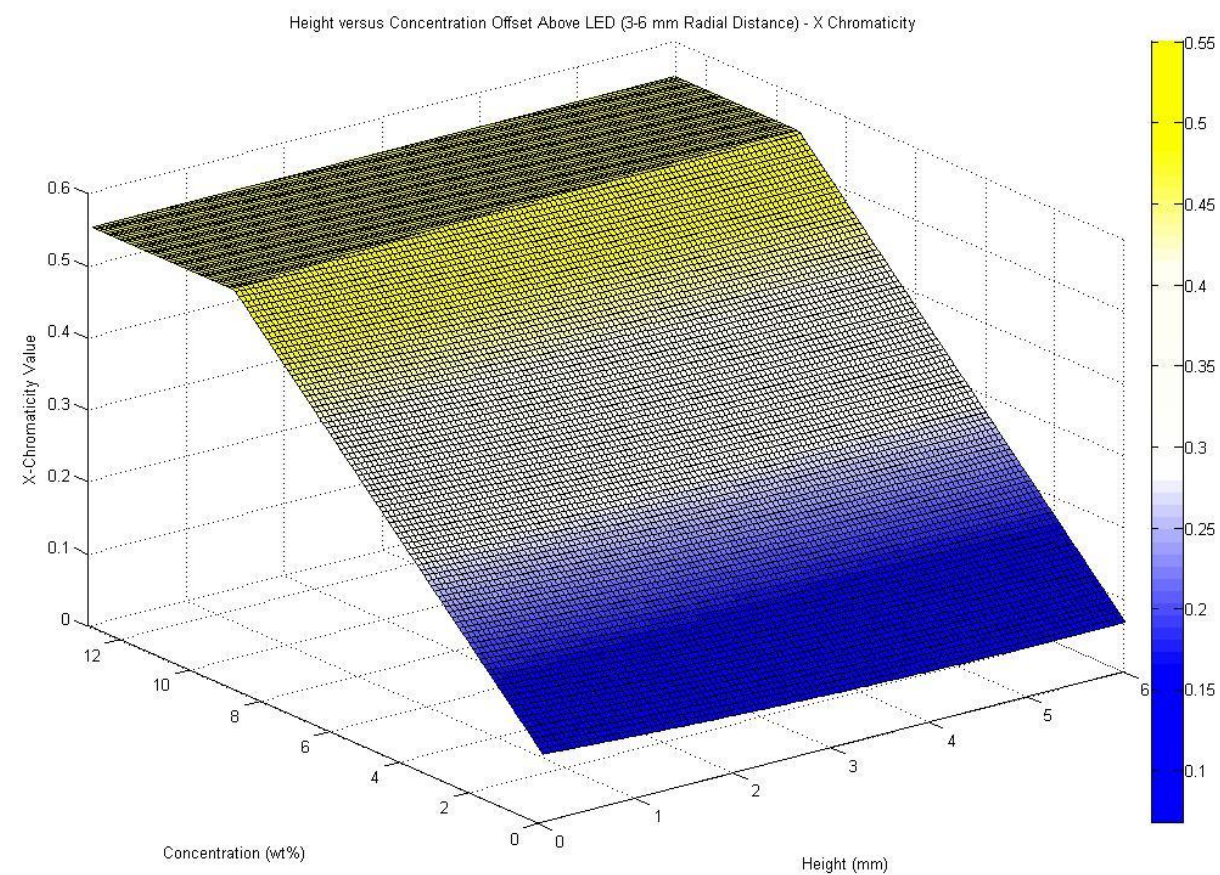

Figure 95 - A 3 dimensional plot color coded to best relay information regarding the actual color of the observed chromaticity values. The plateaus at the bottom and top of the plot are regions which are not valid chromaticity values and therefore are points where the equations fail. This specific plot compares $x$ - chromaticity effects of height and concentration over a 3 to $6 \mathrm{~mm}$ radial distance from the LED. 


\section{Appendix W: Y Chromaticity Plot of Height versus Concentration Offset Above LED}

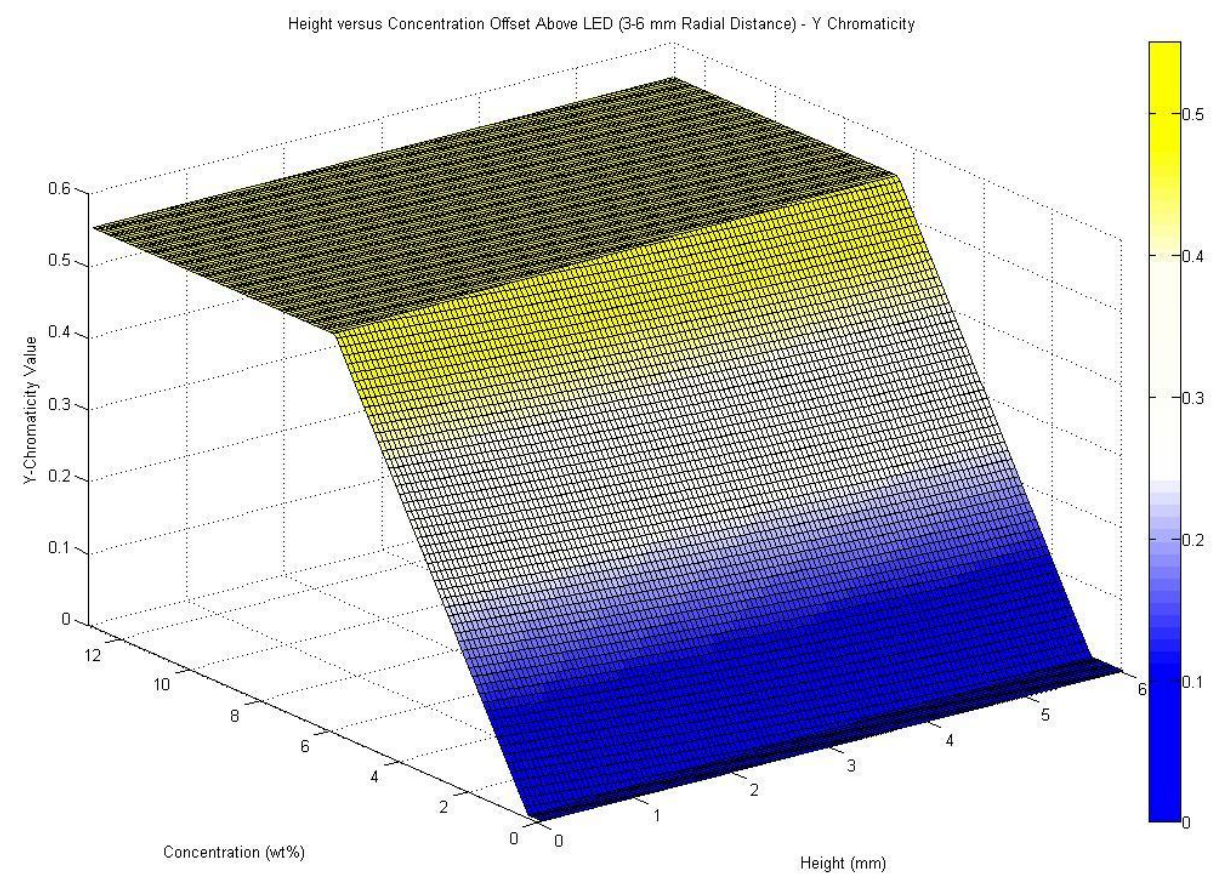

Figure 96 - A 3 dimensional plot color coded to best relay information regarding the actual color of the observed chromaticity values. The plateaus at the bottom and top of the plot are regions which are not valid chromaticity values and therefore are points where the equations fail. This specific plot compares y-chromaticity effects of height and concentration over a 3 to $6 \mathrm{~mm}$ radial distance from the LED. 


\section{Appendix X: Chromaticity Values Measured over a Gaussian}

YAG:Ce/PDMS Lens with LED at 3.58 watts

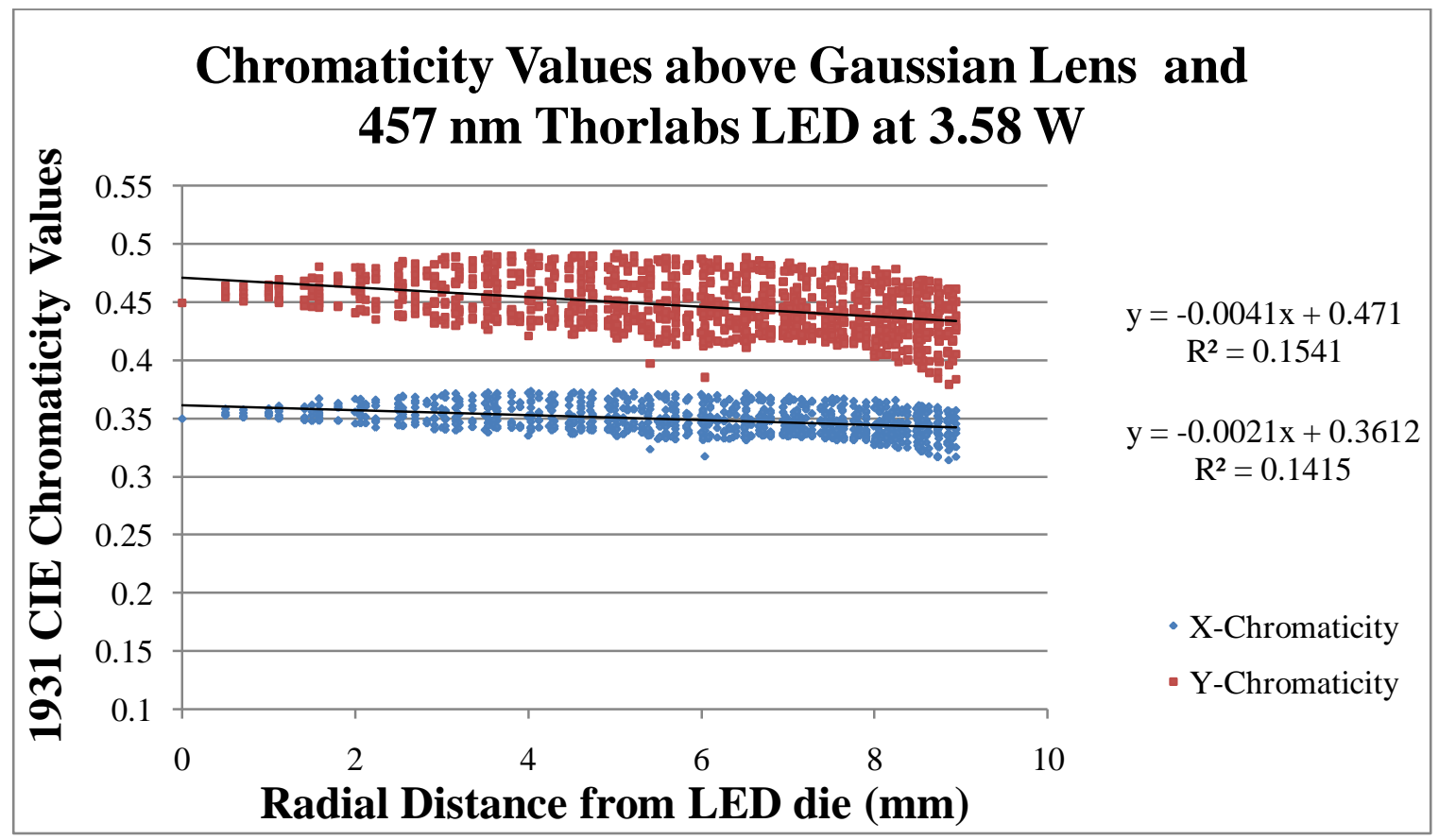

Figure 97 - The change in chromaticity values with radial distance decreases compared to the $4.49 \mathrm{~W}$ tests, however variation for specific radii is greater and chromaticity noise at $10 \mathbf{~ m m}$ is extremely large due to insufficient illumination. 


\section{Appendix Y: Chromaticity Values Measured over a Gaussian}

YAG:Ce/PDMS Lens with LED at 1.51 watts

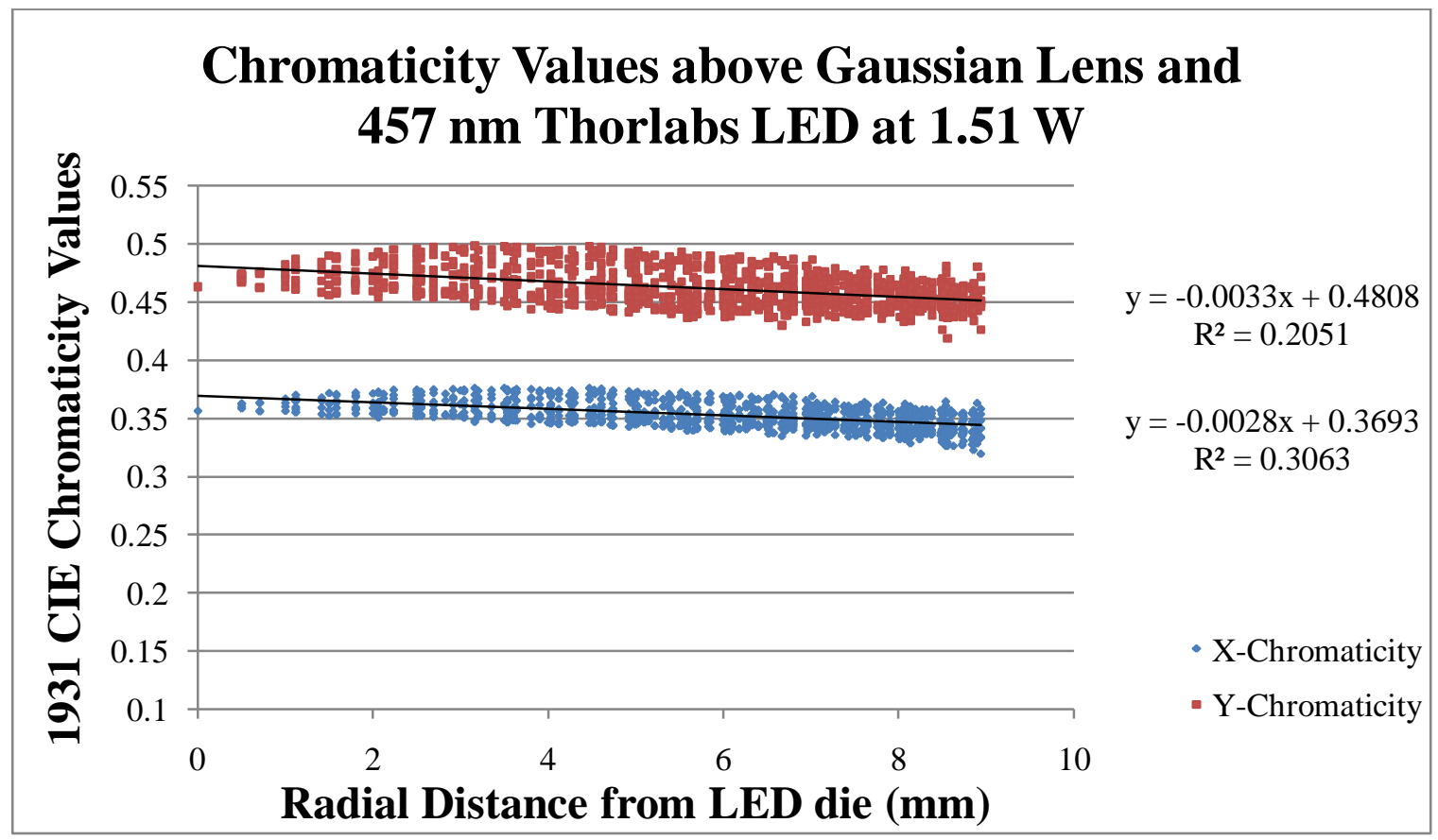

Figure 98 - At only $1.51 \mathrm{~W}$ the luminous emittance is greatly reduced but uniformity is increased over the two other higher power tests for the interval between 0 and $9 \mathrm{~mm}$ radii. 